OBJECT-BASED CLASSIFICATION OF LANDFORMS BASED ON THEIR LOCAL GEOMETRY AND GEOMORPHOMETRIC CONTEXT

A THESIS SUBMITTED TO

THE GRADUATE SCHOOL OF NATURAL AND APPLIED SCIENCES

$\mathrm{OF}$

MIDDLE EAST TECHNICAL UNIVERSITY

BY

DENIZ GERÇEK

IN PARTIAL FULFILLMENT OF THE REQUIREMENTS

FOR

THE DEGREE OF DOCTOR OF PHILOSOPHY

IN

GEODETICAL AND GEOLOGRAPHICAL INFORMATION TECHNOLOGIES

MARCH 2010 
Approval of the thesis:

\section{OBJECT-BASED CLASSIFICATION OF LANDFORMS BASED ON THEIR LOCAL GEOMETRY AND GEOMORPHOMETRIC CONTEXT}

submitted by DENİ GERÇEK in partial fulfillment of the requirements for the degree of Doctor of Philosophy in Geodetic and Geographic Information Technologies, Middle East Technical University by,

Prof. Dr. Canan ÖZGEN

Dean, Graduate School of Natural and Applied Sciences

Assoc. Prof. Dr. Mahmut Onur KARSLIOĞLU

Head of Department, Geodetic and Geographic Information Technologies

Prof. Dr. Vedat TOPRAK

Supervisor, Geological Engineering Dept., METU

Prof. Dr. Josef STROBL

Co-Supervisor, Centre for Geoinformatics, Salzburg University

\section{Examining Committee Members:}

Assoc. Prof. Dr. Zuhal AKYÜREK

Civil Engineering Dept., METU

Prof. Dr. Vedat TOPRAK

Geological Engineering Dept., METU

Prof. Dr. Kadir Dirik

Geological Engineering Dept., Hacettepe U.

Assoc. Prof. Dr. Lütfi SÜZEN

Geological Engineering Dept., METU

Assoc Prof. Dr. Şebnem DÜZGÜN

Mining Engineering Dept., METU

Date: 26. 03. 2010 
I hereby declare that all information in this document has been obtained and presented in accordance with academic rules and ethical conduct. I also declare that, as required by these rules and conduct, I have fully cited and referenced all material and results that are not original to this work.

Name, Last Name: DENIZZ GERÇEK

Signature: 


\begin{abstract}
OBJECT-BASED CLASSIFICATION OF LANDFORMS BASED ON THEIR LOCAL GEOMETRY AND GEOMORPHOMETRIC CONTEXT
\end{abstract}

Gerçek, Deniz

Ph.D., Department of Geodetic and Geographic Information Technologies

Supervisor: Prof. Dr. Vedat Toprak

Co-Supervisor: Prof. Dr. Josef Strobl

March 2010, 202 pages

Terrain as a continuum can be categorized into landform units that exhibit common physiological and morphological characteristics which might serve as a boundary condition for a wide range of application domains. However, heterogeneous views, definitions and applications on landforms yield inconsistent and incompatible nomenclature that lack interoperability. Yet, there is still room for developing methods for establishing a formal background for general type of classification models to provide different disciplines with a basis of landscape description that is also commonsense to human insight.

This study proposes a method of landform classification that reveals general geomorphometry of the landscape. Landform classes that are commonsense to human insight and relevant to various disciplines is adopted to generate landforms at the landscape scale. Proposed method integrates local geometry of the surface with geomorphometric context. A set of DTMs at relevant scale are utilized where local geometry is represented with morphometric DTMs, and geomorphometric context is incorporated through relative terrain position and terrain network. "Object-based image analysis (OBIA)" tools that have the ability to segment DTMs into more representative terrain objects and connect those objects in a multi-level hierarchy is adopted. A fuzzy classification approach is utilized via semantic descriptions to represent ambiguities both in attribute and geographical space. 
Method is applied at different case areas to evaluate the efficiency and stability of the classification. Outcomes portray reasonable amount of consistency where the results can be utilized as general or multi-purpose regarding some ambiguity that is inherent in landforms as well.

Keywords: terrain, general geomorphometry, landforms, DTMs, OBIA, fuzzy classification 


\title{
ÖZ
}

\author{
YERŞEKILLERININ GEOMETRIK VE JEOMORFOMETRIKK \\ ÖZELLIKLERINE GÖRE NESNE TABANLI SINIFLANDIRILMASI
}

Gerçek, Deniz,

Doktora, Jeodezi ve Coğrafi Bilgi Teknolojileri Bölümü

Tez Yöneticisi: Prof. Dr. Vedat Toprak

Ortak Tez Yöneticisi: Prof. Dr. Josef Strobl

Mart 2010, 202 sayfa

Süreklilik gösteren bir yapıya sahip olan arazi çok sayıda uygulama için ayırıcı sınır belirlemekte kullanilabilen ortak fizyolojik ve morfolojik karakteristiklere sahip yerşekillerine kategorize edilebilir. Ancak, yerşekilleri ile ilgili çok çeşitli görüş, tanımlar ve uygulamamalar tutarsız ve birbiriyle karşılaştırılamayan isimlendirme sistemleri ortaya çıkarmıştır. Dolayısıyla, farklı disiplinlerce kullanılabilecek ve aynı zamanda insanlara yabancı olmayan temel arazi tanımlarına dayanan genel sınıflandırma modelleri için formal alt yapılar oluşturmak üzere metodlar geliştirmeye ihtiyaç vardır.

$\mathrm{Bu}$ çalışma, arazinin genel jeomorfometrisini ortaya koyan bir sınıflandırma metodu önermektedir. Bu yüzden, peyzaj ölçeğinde yerşekilleri elde etmek için insanlara anlamlı gelen ve çeşitli disiplinler tarafından kullanılabilir arazi sınıfları esas alınmıştır. Önerilen metod yüzeyin lokal geometrisi ile jeomorfometrik içeriğini entegre etmektedir. Lokal geometri; morfometrik Sayısal Arazi Modelleri (SAM), jeomorfometri ise arazi pozisyonu ve arazi ağı ile temsil edilerek uygun ölçekli SAM setleri üretilmiştir. SAMları araziyi daha iyi temsil eden nesnelere bölümleme (segmentasyon) ve bu nesneleri çok katmanlı hiyerarşide birbiriyle ilişkilendirerek sınıflandırma yeteneğine sahip nesne tabanlı görüntü analizi teknikleri kullanılmıştır. Semantik tanımlamalar ve bulanık mantık ile sınıflandırma yaklaşımı kullanılarak hem öznitelik hem de mekansal boyuttaki belirsizlikler temsil edilmeye çalışılmıştır. 
Metod, sınıflandırmanın verimliliğini ve stabilitesini test etmek için farklı çalışma alanlarında uygulanmıştır. Sonuçlar makul düzeyde tutarlılık göstermiştir ve arazi formlarının kendine has belirsizlik durumu da göz önünde bulundurularak genel veya çok amaçlı kullanımlar için uygun bulunmuştur.

Anahtar kelimeler: arazi, yerşekilleri, genel jeomorfometri, Sayısal Arazi Modelleri (SAM), nesne tabanlı görüntü analizi, bulanık sinıflandırma 
To My Family 


\section{ACKNOWLEDGEMENTS}

For his guidance, continuous support and encouragements throughout this study, I express my sincerest thanks and deepest respect to my supervisor Prof. Dr. Vedat Toprak. He has always been very patient to me during the course of this study. I feel very lucky to have worked with him and to be acquainted with his wisdom. He is more than a supervisor to me.

I would like to thank sincerely to my co-supervisor Prof. Dr. Josef Strobl for his continuous support, guidance and valuable contributions to this study. I am in dept to him for providing me with a workspace for my studies in Centre for Geoinformatics (Z_GIS) in Salzburg, Austria which contributed a lot to this study. I appreciate him for devoting his valuable time whenever I asked for his guidance. I am very happy to know an academician, a manager and a person like him.

I would like to thank to Dr. Lucian Drăguț for his support and valuable suggestions. Discussions with him and Clemens Eisank made many contributions to this study. I would also like to thank them both for their evaluations on the results. It is a pleasure for me to have people working on similar topics as friends.

Assoc. Prof. Dr. Bora Rojay and Dr. Jochen Schmidt are also gratefully thanked for making evaluations on the results.

For his technical support, I would like to thank Dr. Dirk Tiede.

I would like to express my special thanks to my committee member Assoc. Prof. Dr. M.Lütfi Süzen for his contributions and continuous support throughout this study. I would also like to thank to my committee member Assoc Prof Dr. Zuhal Akyürek for her suggestions. My other committee members; Prof. Dr. Kadir Dirik and Assoc. Prof. Dr. Şebnem Düzgün are also gratefully acknowledged. 
I would like to thank Definiens team for providing me with a short term academic license of the professional version of software: 'eCognition' for free which made available the partial fulfilment of this study.

I acknowledge OYP programme for employing me and providing me support during my studies.

My friend Leyla Kamoy is gratefully acknowledged for her contributions for the formatting of the thesis and Ezgi Ünal as well.

Moreover, I would like to express my special thanks to Cağrı Raydemir. He could always take my irritating moods easy during the course of this study and even blown them away. Serdar Ahçı is thanked for his friendship and hospitality, I new I could knock his door whenever I feel stressed and need to chat. Umut Karamahmut is thanked for his friendship. Naci Başocak is thanked for his motivations during the course of this study.

Finally, I would like to express my gratefulness to my family; my father Ahmet, my mother Raife, and my brother Cem Özgür. I should thank them sincerely for their patience and continuous support which made available the fulfilment of this work. 


\section{TABLE OF CONTENTS}

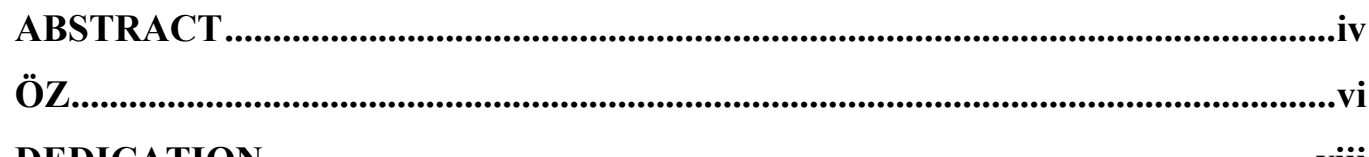

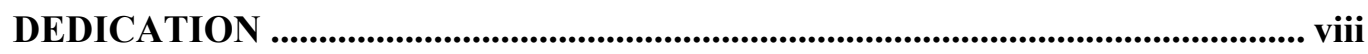

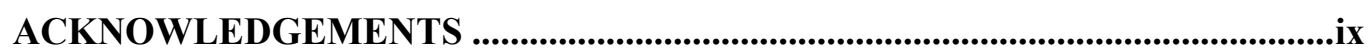

TABLE OF CONTENTS ...................................................................................

LIST OF TABLES ..........................................................................................

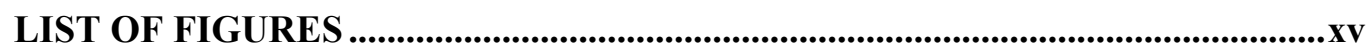

\section{CHAPTERS}

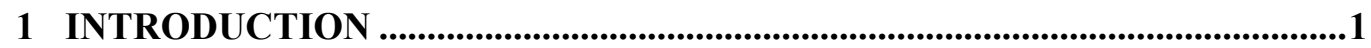

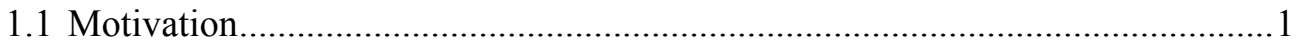

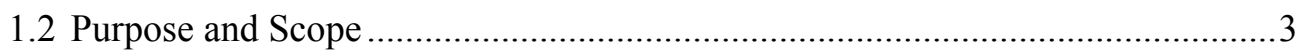

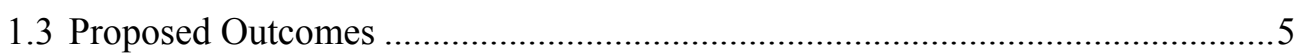

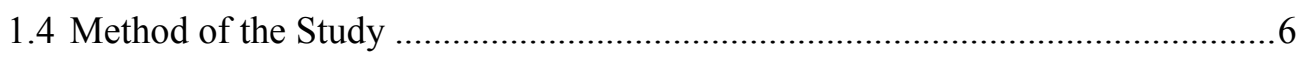

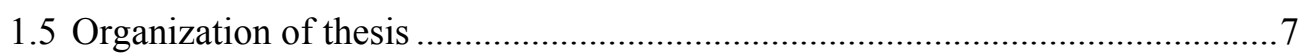

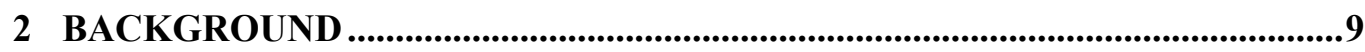

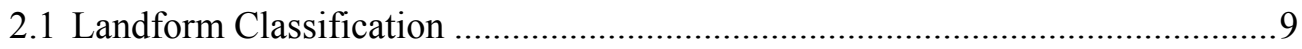

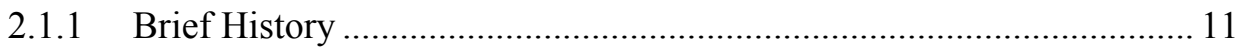

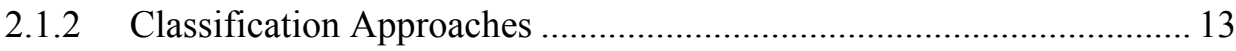

2.1.2.1 Classification based on Specific Geomorphometry .................. 14

2.1.2.2 Classification based on General Geomorphometry .................. 15

2.1.3 Automated Landform Classification.................................................... 16

2.1.4 Trends in automated landform classification ....................................... 17

2.1.4.1 Semantically oriented vs. data-driven classification.................. 17

2.1.4.2 Fuzzy vs. Crisp Classifications ................................................ 19

2.1.4.3 Object-based vs Pixel-based Approaches .................................. 20

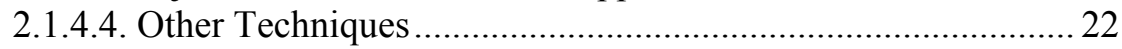

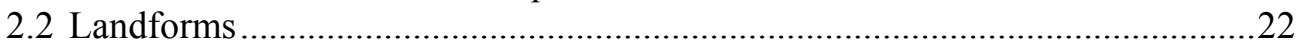

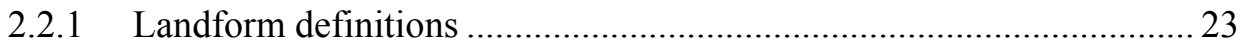

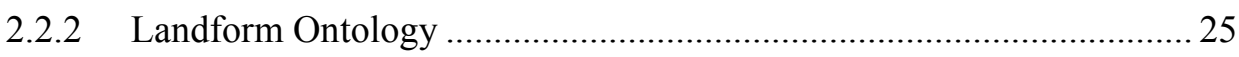

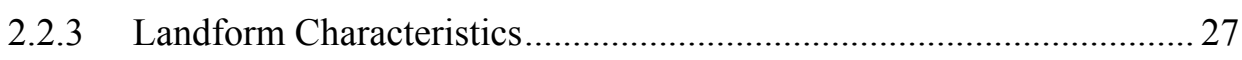

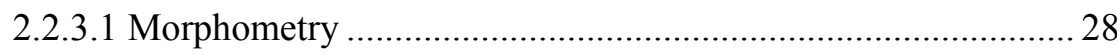




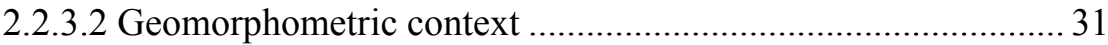

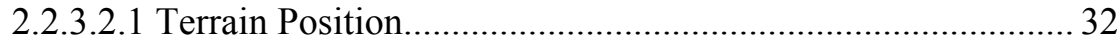

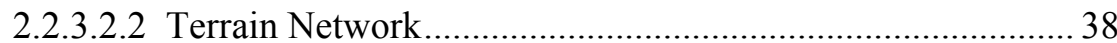

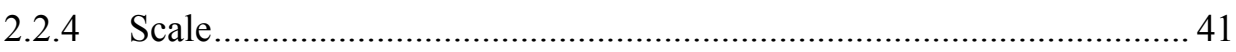

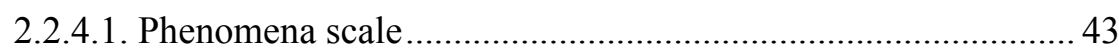

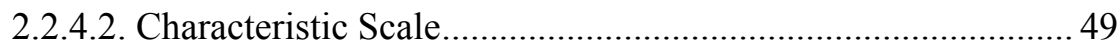

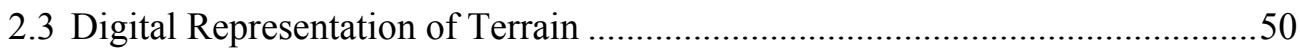

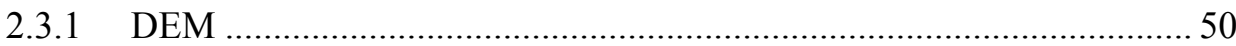

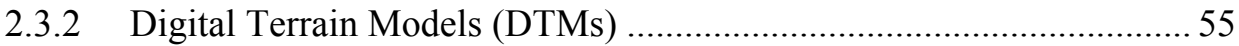

2.3.2.1 Morphometry/Local Geometry .................................................5 56

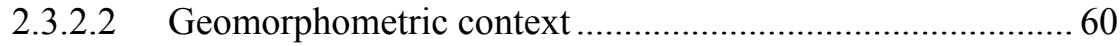

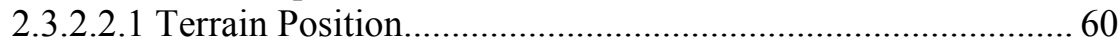

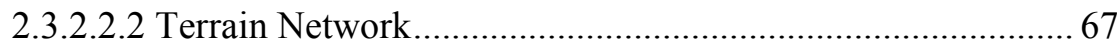

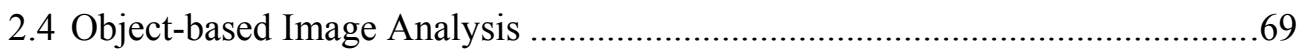

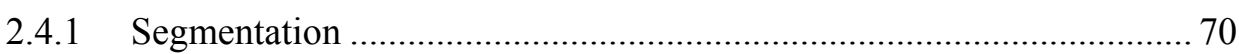

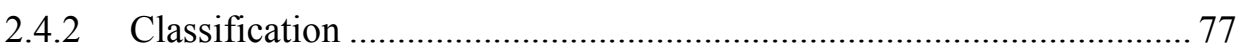

3 METHOD OF LANDFORM CLASSIFICATION ..............................................79

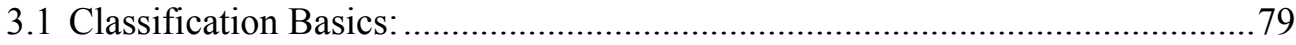

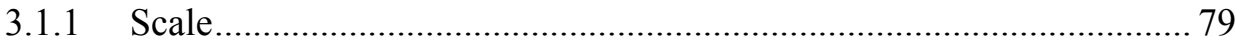

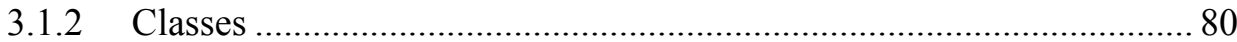

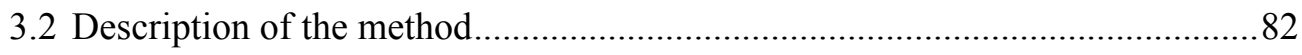

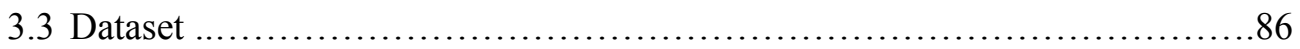

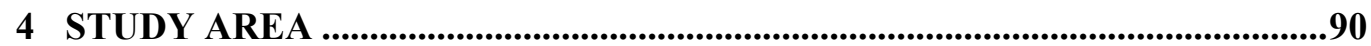

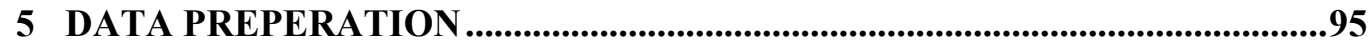

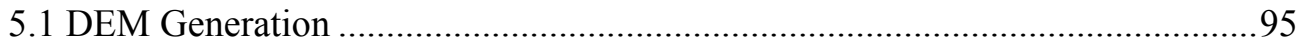

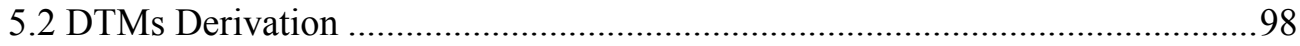

5.2.1 Morphometry /Local Geometry ……..................................................... 98

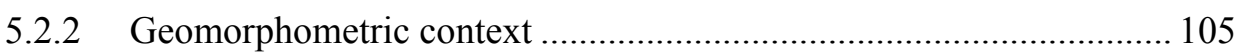

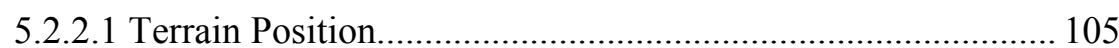

5.2.2.2 Terrain Network.............................................................. 107

6 OBJECT-BASED CLASSIFICATION OF LANDFORMS ...............................112

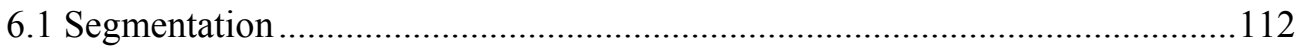

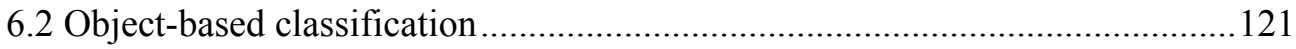

6.2.1 Classification of landforms based on local geometry .............................. 121

6.2.2 Classification based on terrain position ................................................ 135

6.2.3 Classification based on Geomorphometric Context............................... 136

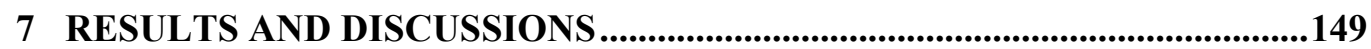




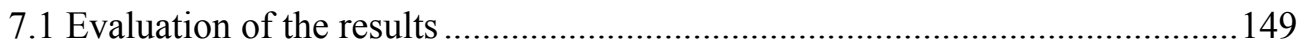

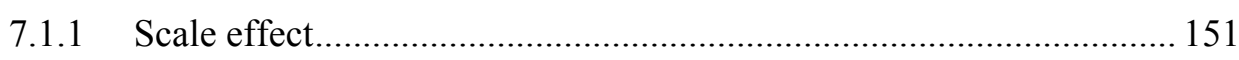

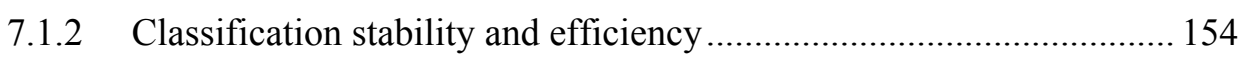

7.1.3 Geomorphometric context ............................................................ 159

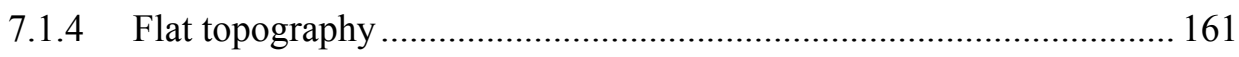

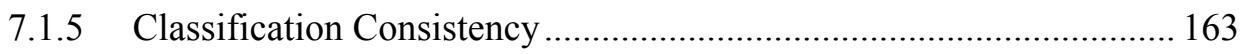

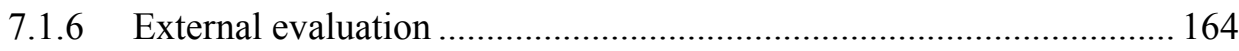

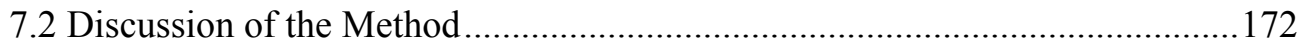

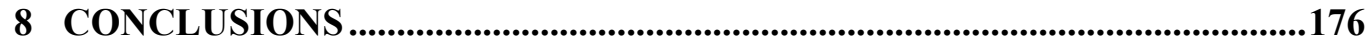

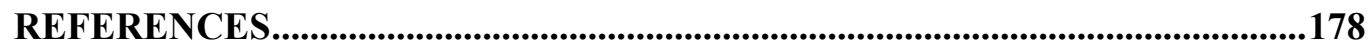

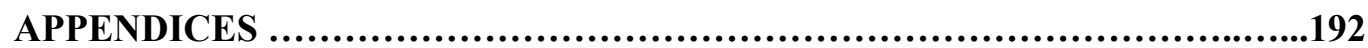

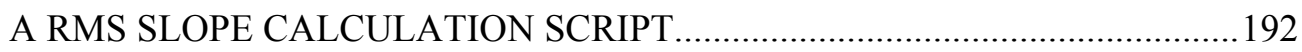

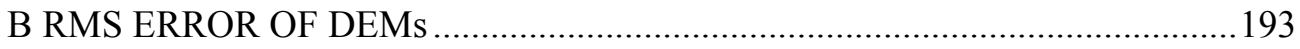

C LANDSERF CHARACTERISTIC SCALE BY WOOD (2009) …....................194

D ESP RESULTS: RATE OF CHANGE (ROC) OF LV ......................................195

E STEPS FOR NORMALIZATION OF DTMs …………...................................196

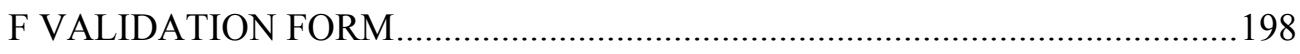

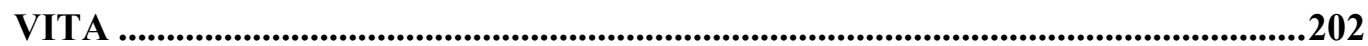




\section{LIST OF TABLES}

Table 2.1: Landform units defined by Conacher and Dalrymple (1977) ............................ 34

Table 2.2: Fifteen landform units proposed by MacMillan and Pettapiece (2000)............... 36

Table 2.3: Topographic position classes by Speight (1990).............................................. 37

Table 2.4: Dimensions of relief units at hierarchical levels and corresponding examples of

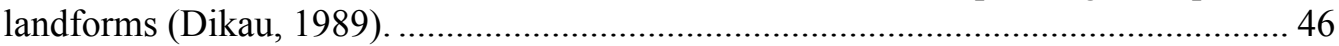

Table 2.5: Attributes of hierarchical levels of relief units: Microrelief and Mesorelief (Dikau, 1992).

Table 3.1: List of different case studies, their intention of being exericised, classification method employed and their relevant scales as DTM window size and data sets ......... 87

Table 4.1: Vicinity, coordinates and extents of case areas: case1, case1next and case2 ...... 93

Table 4.2: Summary statistics of case areas: case1, case1 next and case 2 ........................... 94

Table 5.1: DEM RMS error for 'case1'(top) and 'case2'(bottom) ...................................... 98

Table 5.2: Summary statistics; mean and standard deviation for maximum curvature convexity calculated for a range of window sizes................................................... 102

Table 5.3: Multiple scales of datasets and their corresponding cases................................ 103

Table 6.1: Segmentation parameters for all cases.......................................................... 118

Table 6.2: Summary statistics for morphometric DTMs of case areas: 'case1fine', case1, 'case1 coarse', 'case1next', 'case2'................................................................... 124

Table 6.3: A list of operators to utilize information from several membership functions. . 127

Table 6.4: Topographic Position Index (TPI) value ranges for case areas: 'case1',

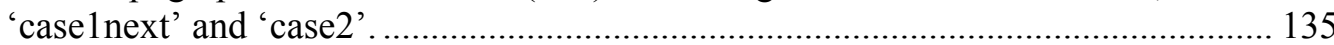

Table 7.1: The percentage of areas of objects at three scales of case1; 'case1 fine', 'case1',

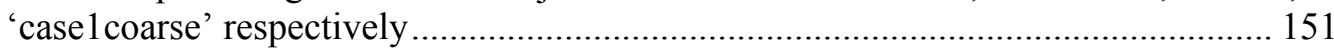

Table 7.2: Classification stability and best classification results for morphometric classification of 'case1fine' sorted in descending order

Table 7.3: Classification stability and best classification results for morphometric classification of 'case1' sorted in descending order.

Table 7.4: Classification stability and best classification results for morphometric classification of 'case1 coarse' sorted in descending order

Table 7.5: Classification stability and best classification results for morphometric classification of 'case 2 and 'case1'

Table 7.6: 'change matrix' for morphometric classification vs geomorphometric classification of 'case1'

Table 7.7: Evaluation results compared with suggestions from four scientists for case1 Exact matches are highlighted in yellow, close matches are highlighted in fair yellow and total mismatch is with red font

Table 7.8: Evaluation results compared with suggestions from four scientists for case2. Exact matches are highlighted in yellow, close matches are highlighted in fair yellow and total mismatch is with red font

Table B.1: Elevation values of sample Ground Control points compared to corresponding grid elevations at varying resolutions of DEMs for case 1

Table B.2: Elevation values of sample Ground Control points compared to corresponding

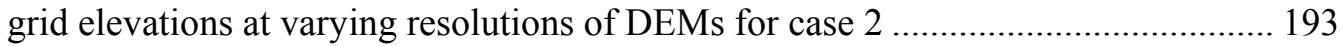

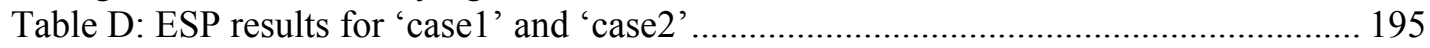




\section{LIST OF FIGURES}

Figure 2.1: Six Landform classes offered by Peucker and Douglas (1974).......................... 29

Figure 2.2: Fifteen 'form elements' offered by Dikau (1989) based on local geometry. ..... 30

Figure 2.3: Shary's system of landform classification according to signs of tangential, profile, mean, difference and total Gaussian curvatures (Shary et al., 2005).............. 31

Figure 2.4: a) Slope classification of Wood (1942) b) Slope model by Dalrymple et al.,

Figure 2.5: Geomorphic units offered by Ruhe and Walker (1968). (SU: summit, SH:

shoulder, BS: backslope, FS: foot slope, TS: toe slope, AL: alluvium).
(in (2000)).

Figure 2.7: Basic slope shapes with surface flow lines (Huggett 1975), source: Ventura and Irvin (2000).

Figure 2.8: Geomorphic units offered by Pennock et al. (1987).

Figure 2.9: (a) Ridges and channel lines of a topographic surface (number in parentheses indicate altitude of the point) (B) Tripartite graph representing the topological structure of the surface; edge weights are indicated on the edges (Wolf, 1991).....

Figure 2.10: Terrain topology on a contour map by Schneider (2003). A drainage area is highlighted.

Figure 2.11: Changing morphometric classes with scale (Fisher et al., 2005) ...................... 42

Figure 2.12: Hierarchy of geomorphographic units of differing complexity derived from hierarchy theory (Dikau, 1992).

Figure 2.13: Landform scales depicting nestedhierarchy in Switzerland (Rasemann et al., 2004).

Figure 2.14: Corresponding scales in hydrology and geomorphology (Anderson and Burt, 1990 in Schmidt, 2000).....

Figure 2.15: Hierarchy of landform entities (MacMillan et.al, 2004). ............................... 48

Figure 2.16: Effect of window size enlargement on a specific location on a hilly terrain.... 49

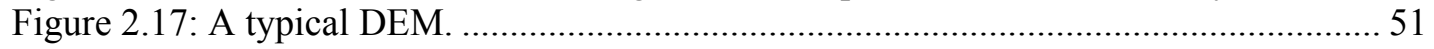

Figure 2.18: Normal sections of four curvatures marked on a hypothetical surface ' $\mathrm{S}$ '....... 58

Figure 2.19: Elevation values in a $3 \times 3$ local window from $Z 1$ to $Z 9$. .................................59

Figure 2.20: a) surface water flow patterns on a planar inclined surface b) surface water flow on a surface with significant plan curvature leading to convergence and divergence of flow paths. Size of arrows indicate the flow accumulation at that point on the surface (Pennock, 2003).

Figure 2.21: Magnitudes of Strahler and Sheve stream orders........................................... 68

Figure 2.22: Flowchart of segmentation based on Fractal Net Evaluation Algorithm by Baatz and Schape (2004). 72

Figure 2.23: Segmentation of seed pixels into objects at a particular scale......................... 73

Figure 2.24: Segmentation with increasing scale parameter on a subset of satellite band a) original band b) segmentation at scale: $5 \mathrm{c}$ ) segmentation at scale: 10 segmentation at scale: 20 .

Figure 2.25: Pixels are merged into objects given a homogeneity/heterogeneity criteria defined by scale. 
Figure 2.26: A typical multi-level hierarchical network of objects with interrelationships and sub-level, super-level relationships.

Figure 2.27 Description of classification rules a) in Sementic Import (SI) Model, b) in similarity relation model via training samples.

Figure 3.1: Representative features that pertain to non-sloping part of the land surface...... 81

Figure 3.2: Representative features that pertain to non-sloping part of the land surface...... 81

Figure 3.3: Flowchart of the proposed landform classification method. ............................. 82

Figure 3.4: A section from the main flowchart which describes classification based on local geometry /morphometric classification....

Figure 3.5: A section from the main flowchart which describes terrain position classification

Figure 3.6: A section from the main flowchart which describes geomorphometric classification.

Figure 3.7. Shaded reliefs of the study areas 'case1', 'case1next' (left) and 'case2' (right) 86

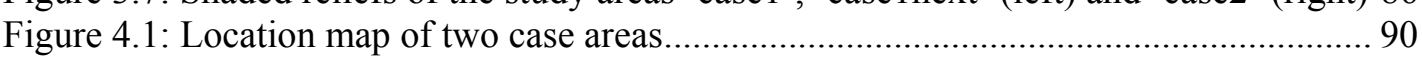

Figure 4.2: Study area: shaded relief map of 'case1'. For location see Figure 4.1 .............. 91

Figure 4.3: Study area: shaded relief map of 'case2'. For location see Figure 4.1 …........... 92

Figure 4.4:. Study area: shaded relief map of 'case1next' that overlap with 'Case1'. 8x8 km overlap to be studied in classification evaluation is shown......................................... 92

Figure 4.5: Hypsometric curves of two case areas: case 1, case 2 ...................................... 94

Figure 5.1: DEM for case1 (bigger scene on the left) and 3 by $3 \mathrm{~km}$ samples of shaded relief for $10 \times 10$ to $30 \times 30$ m resolution DEMs.

Figure 5.2: DEM for case2 (bigger scene on the left) and 3 by $3 \mathrm{~km}$ samples of shaded relief

for 10x10 to 30x30 m resolution DEMs................................................................... 97

Figure 5.3: Shaded relief of case1, maximum curvature over a range of windows sizes; .. 100

Figure 5.4: Characteristic scale results for window sizes from $3 \times 3$ to $25 \times 25 \ldots \ldots \ldots \ldots \ldots . . . .101$

Figure 5.5: Characteristic scale results for window sizes from $3 \times 3$ to $45 \times 45$................... 101

Figure 5.6: Characteristic scale results for window sizes from $3 \times 3$ to $85 \times 85$.................. 101

Figure 5.7: Varying window sizes imposed over a curved feature.................................. 102

Figure 5.8: Graph for mean for maximum curvature convexity calculated for a range of

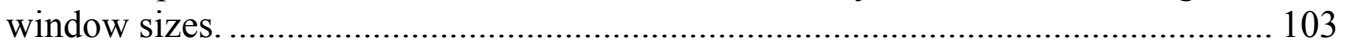

Figure 5.9: Location of $3 \times 3 \mathrm{~km}$ sample area in the mid part of the study area case 1........ 104

Figure 5.10: Morphometric DTMs of $3 \times 3 \mathrm{~km}$ sample area for case 1 calculated at scale $15 \times 15$.

Figure 5.11: Morphometric DTMs of $3 \times 3 \mathrm{~km}$ sample area for case 1 calculated at scale $45 \times 45$

Figure 5.12: Morphometric DTMs of 3x3km sample area for case 1 calculated at scale

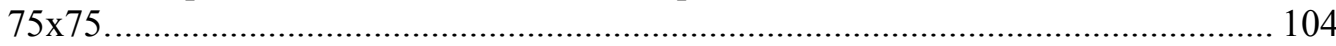

Figure 5.13: $3 \times 3 \mathrm{~km}$ sample area in the mid part of the study area case 2..................... 105

Figure 5.14: Morphometric DTMs of $3 \times 3 \mathrm{~km}$ sample area for case 3 calculated at scale

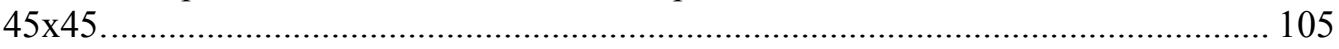

Figure 5.15: Ridge to stream distances for case1 (on the left) and case2 on the right........ 106

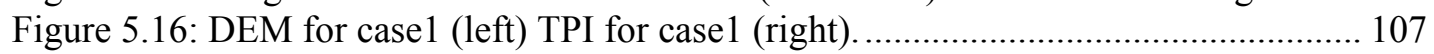

Figure 5.17: DEM for case2 (on the left) TPI for case1 (on the right). ............................ 107

Figure 5.18: Case1 (left), streams of Strahler order (right). Light blue to dark blue represents

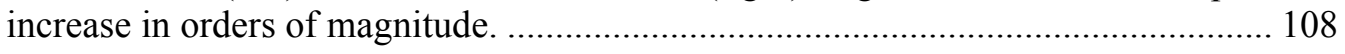

Figure 5.19: Watersheds and basins (on the left), inverse DEM streams (on the right) for case 1 .

Figure 5.20: Streams (left), ridges (right) used as terrain network features in geomorphometric classification of case1.

Figure 5.21: Case1 (left), streams of Strahler order (right). Light blue to dark blue represents increase in orders of magnitude. 
Figure 5.22: Watersheds and basins (on the left), inverse DEM streams (on the right) for case 2

Figure 5.23: Streams (left), ridges (right) used as terrain network features in geomorphometric classification of case2.

Figure 6.1: Hierarchical multi-level segmentation proposed for geomorphometric classification

Figure 6.2: Schematic representation of segmentation parameters (eCognition Developer v.8, Reference Book).

Figure 6.3: Rate of change (ROC) of local variance (LV) of slope calculated over a range of scales (1-20) for 'case1 fine'via ESP tool

Figure 6.4: Rate of change (ROC) of local variance (LV) of slope calculated over a range of scales (1-20) for 'case1' via ESP tool

Figure 6.5: Rate of change (ROC) of local variance (LV) of slope calculated over a range of scales (1-20) for 'case1 coarse' via ESP tool

Figure 6.6: Rate of change (ROC) of local variance (LV) of slope calculated over a range of scales (1-20) for "case2" via ESP utility

Figure 6.7: Relief map of case 1 with a sample area of $3 \times 3$ in the middle (a); segmentation results for 'case1fine' (b); case1 (c), 'case1 coarse' (d) illustrated for the sample area.

Figure 6.8: Morphometric DTMs of 3x3km sample area for 'case1 fine' with relevant segmentation overlaid

Figure 6.9: Morphometric DTMs of $3 \times 3 \mathrm{~km}$ sample area for 'case1' with relevant segmentation overlaid.

Figure 6.10: Morphometric DTMs of 3x3km sample area for 'caselcoarse' with relevant segmentation overlaid.

Figure 6.11: Relief map of 'case2' with a sample area of $3 \times 3$ in the middle (a) and segmentation results for 'case2' illustrated for the sample area of $3 \times 3 \mathrm{~km}$............... 120

Figure 6.12: Morphometric DTMs of 3x3km sample area in the middle for 'case2' with relevant segmentation overlaid.

Figure 6.13: Bell shaped, $z$-shaped, s-shaped membership functions, $a$ and $b$ are the threshold limits.

Figure 6.14: Set of membership functions that can be utilized in classification in eCognition software (eCognition Developer v.8, Reference Book).

Figure 6.15: Fuzzy membership functions used as Semantic Import (SI) model for the study.

Figure 6.16: Membership functions that are used to import semantics on curvedness (top) and straightness (bottom).

Figure 6.17: Membership functions that are used to import semantics on steepness vs. flatness.

Figure 6.18: Schematic representation of membership operator of form descriptors employed for the study.

Figure 6.19: Fuzzy membership functions used for describing non-sloping forms.............. 129

Figure 6.20: Fuzzy membership functions used for describing non-sloping forms............ 130

Figure 6.21: Morphometric classification result for 'case1 fine' ..................................... 131

Figure 6.22: Morphometric classification result for 'case1' ............................................ 132

Figure 6.23: Morphometric classification result for 'case1 coarse' ................................... 133

Figure 6.24: Morphometric classification result for $3 \times 3$ sample area for 'case1fine', 'case1' and 'casel coarse'.

Figure 6.25: Hierarchical multi-level classification proposed for the classification............ 137

Figure 6.26: Conditions for an object to be classified as 'peak' ....................................... 138

Figure 6.27: Logical rule set for geomorphometric classification of 'peak' ...................... 139

Figure 6.28: Logical rule set for geomorphometric classification of 'ridge'...................... 139

Figure 6.29: Logical rule set for geomorphometric classification of 'saddle' .................... 139 
Figure 6.30: Logical rule set for geomorphometric classification of 'plain' .

Figure 6.31: Logical rule set for geomorphometric classification of 'channel' .................. 140

Figure 6.32: Logical rule set for geomorphometric classification of 'pit' ........................... 140

Figure 6.33: Logical rule set for geomorphometric classification of 'nose' ........................ 140

Figure 6.34: Logical rule set for geomorphometric classification of 'spur' ....................... 141

Figure 6.35: Logical rule set for geomorphometric classification of 'spur foot'................ 141

Figure 6.36: Logical rule set for geomorphometric classification of 'shoulder'. .................. 141

Figure 6.37: Logical rule set for geomorphometric classification of 'planar slope'............ 142

Figure 6.38: Logical rule set for geomorphometric classification of 'foot slope'. ............. 142

Figure 6.39: Logical rule set for geomorphometric classification of 'hollow shoulder' . ... 142

Figure 6.40: Logical rule set for geomorphometric classification of 'hollow'. .................. 143

Figure 6.41: Logical rule set for geomorphometric classification of 'hollow foot'............. 143

Figure 6.42: Geomorphometric classification result for 'case1' ...................................... 144

Figure 6.43: 3D views of Geomorphometric classification result for 'case1' ..................... 145

Figure 6.44: Geomorphometric classification result for 'case2' ........................................ 146

Figure 6.45: 3D views of Geomorphometric classification result for 'case2' .................... 147

Figure 6.46: Overlapping area classified as a part of morphometric classification of 'case1next' .......................................................................................... 148

Figure 7.1 Total area of per class of morphometric classification for 'case1 fine', 'case1' 152 and 'case1coarse'. First six of the classes pertain to non-sloping and other nine classes

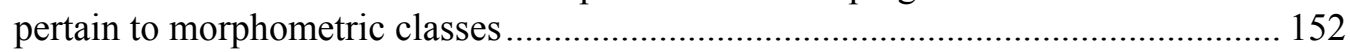

Figure 7.2: Spatial distribution of the intersecting morphometric classes in 'case1' ......... 152 and 'case1fine' (left), all cases (middle), 'case 1' and 'case1 coarse'(right)...................... 152

Figure 7.3 Percent of difference from fine to coarse resolution: case1 fine to case coarse 153

Figure 7.4: Percent of difference from fine to coarse resolution: case 1 to case coarse ...... 153

Figure 7.5: classification stability of three scales of morphometric classification of case 1157

Figure 7.6: Best classification result of three scales of morphometric classification of case1

Figure 7.7: Classification stability results for morphometric classification of "case 2 and 'case1'

Fgure 7.8: Classification stability results for morphometric classification of 'case 2 and 'case1'

Figure 7.9: 3D plot of 'change matrix' for morphometric classification vs. geomorphometric

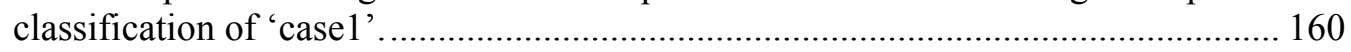

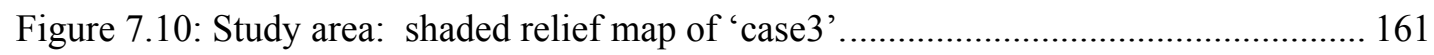

Figure 7.11: Geomorphometric classification result for 'case3' ...................................... 162

Figure 7.12: Overlapping area classified as a part of morphometric classification of 'case1next' (left) and 'case1' (right)..................................................................... 164

Figure 7.13: Stratified random points on case areas: 'case1'(left) and, 'case2' (right)..... 165

Figure 7.14: representation of anticipated landforms across the landscape. Plain ant pit are not inline with particular organisation ................................................................ 170

Figure 7.15: An anticipated weights of similarity of landform classes............................. 171

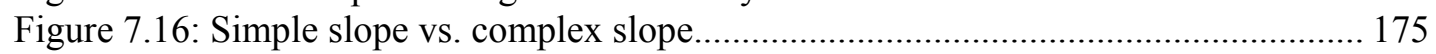




\section{CHAPTER 1}

\section{INTRODUCTION}

\subsection{Motivation}

There are many reasons that justify the need to reduce complexity of terrain by delineating it into relatively homogeneous areas. Those needs can be attributed to human cognition at the first place; multiplicity of objects, properties of them and relationships between them in the material world that we live in is better managed by means of categorization (Lakoff, 1987). Therefore, terrain classification, like any other categorization attempt by human is intrinsic. It is more likely that we can understand what the categories of land reveal, than to perceiving and evaluating continuous representations. Moreover, there is a long tradition of mapping which can be attributed to the relative ease of representing discrete spatial units compared to understanding and evaluating continuous representations of surface (Strobl, 2007).

Variability of land properties is mainly controlled by changes in form and position of terrain that produce gradients in moisture, energy and nutrients across the landscape (Band et al., 1991; Moore et al., 1993; Skidmore et al., 1996). Therefore, delineation of landform elements that functionally and morphologically represent the earth surface land has occupied the minds of earth scientists and planners for decades.

In the literature of terrain modelling, analysis and quantification, there exists a vast amount of inconsistent and incomparable nomenclature (Pike, 1988). This is because terrain classification is usually made in different intentions and every single research may have its own motivations and reasons to produce a map. For the case of landform classification; there are very heterogeneous views, definitions and applications. Various authors have proposed notations on landforms (Hammond, 1964; Dalrymple et al., 1968; Peucker and 
Douglas; 1974; Speight, 1974, Pennock et al., 1987; Zuidam, 1986; Dikau, 1989); however there are no unique and universal definitions for them. Due to this heterogeneity in discipline perception world for landforms (Bishr, 1998, Dehn, 2000), problems arise in exchange of information, interoperability of the maps among users with different contexts in the background.

Nevertheless, terrain still has components that can be explicitly and neutrally described. It is, for example, certainly expected that a 'hollow' is a concave part of the earth surface, whereas a 'spur' has a convex form and both are on the sloping part of a surface. Therefore, a fundamental property of landforms is related to local geometry (Zuidam, 1986; Dikau ,1989; Schmidt and Hewitt, 2004), hence morphometry. Moreover, local geometry / morphometry or simply form is very common to disciplines involved in terrain analysis since all major approaches representing landforms treat land surface as composed of smaller form elements (Dehn et al., 2001, MacMillan et al. 2004). However, form refers to a general condition and may pertain to any object even outside land surface, therefore, landforms need further concern as they are not neutrally labelled in line with their geometry but regarding their geomorphometry as well, e.g.. a 'ridge' is at a relatively higher position across the landscape and it typically overlaps with basin divides, therefore a ridge is not only described with its local geometry but also a higher geomorphometric context (Schmidt and Hewitt, 2006).

General type of classification schemes (Evans, 1972) that avoid subjective definition and delimitation of surfaces (Evans, 1990) is adequate for making general and neutral descriptions of land surfaces. However, they are less common compared to vast amount of specific classification schemes. Because, research and applications are usually oriented in solving a problem or implementing requirements of an application domain where the classification attempts often ends up with products that have restricted use and lack interoperability.

Although there are published research on 'general geomorphometry' and attempts that materialize those approaches, there is still room for developing methods for establishing a formal background for general type of classification models and utilize new techniques that better represent topography,

Object-based Image Analysis (OBIA) combining powerful characteristics to segment images into objects and building relationships between them is currently being evaluated by 
several authors for landscape classification. OBIA tools provide promising utilities for representing landforms in object domain within framework of neighbourhood and hierarchical relationships that is very particular to landforms.

This research focuses on utilizing Object-Based Image Analysis (OBIA) methods for producing a general type of landform classification rather than a specific or on purpose, to provide different disciplines with a basis of landscape description that is also commonsense to human insight.

\subsection{Purpose and Scope}

Emphasizing the importance of interoperability and easy exchange of information, this study aims to produce landform classes that are commonsense to human insight and appropriate or transferable for use of various disciplines. This study tries to keep away from particular description and limitations introduced by different discipline perceptions. Hence a 'general' classification scheme (Dikau, 1989) that reveals general geomorphometry of the landscape is proposed. This scheme describes general geomorphometry and involves a set of 15 generic and exhaustive landforms that can be utilized as general or multi-purpose.

Geometric form constitutes the basis of the proposed classification method where geomorphometric context is incorporated to yield landform classes which pertain to land surface. General geomorphometry involves quantitative characterization of any surface even outside land surface (Wood, 1996); hence form elements gathered based on local geometry should be integrated with geomorphometric context. Classification approaches integrating local geometry and geomorphometric context have proved to yield promising results (Schmidt and Dikau, 1999; MacMillan et al., 2004; Scmidt and Hewitt, 2006; Dragut and Blaschke, 2006). Proposed method integrates local geometry of the surface with geomorphometric context that is represented by relative terrain position and terrain network features.

Landform classification attempts have a long history dating back to mid 18th century with the availability of maps and aerial photographs. Conventional techniques of landform delineation which are based on visual stereo aerial photo interpretation and measurements on topographic maps are subject to expert bias and they extensively lack interoperability, i.e.; variation in the application or interpretation of rules, differences between surveyors, 
suggests that there is room for reproducible and generic approaches to the identification and delineation of landforms (Burrough et al., 2000). Fortunately now it is extensively possible with the advent of computing resources, powerful GIS tools for spatial analysis and widely available, e.g. digital terrain data even at $30 \mathrm{~m}$ publicly accessible i.e. SPOT Image. Landform classification method proposed generates and utilizes quantitative representations of terrain surface and classify terrain in an automated fashion that is robust and reproducible and less prone to subjectivity and bias.

Even though there have been efforts to totally recover from subjectivity in landform classification incorporating statistical clustering techniques (e.g. Irvin et al., 1997; Burrough et al., 2000; Macmillan et al., 2000), there is a tendency of using semantically oriented techniques more recently (Dehn et al., 2001; Schmidt and Hewitt, 2004, Dragut and Blaschke, 2006; Prima et al., 2006). Multivariate terrain data do not usually portray significant clusterings that correspond to particular landforms, as landforms ontologically are not 'real' objects with determinate distinctions, like for instance land cover type 'deciduous forest" compared to its surroundings has. Therefore a semantically oriented /supervised classification technique with the implementation of Semantic Import (SI) model that produces fuzzy landform elements is adopted for this study. Terrain representations depict a multivariate continuum that has little or no distinct class-boundaries as there is an inherent overlap between different classes, in both spatial and attribute space (Burrough and Frank, 1996, Fisher et al., 2004; Romstad, 2001). Therefore it is necessary to make a continuous classification that represent smooth transition of the landform classes and their indeterminate boundaries. Class definitions for this study were built via Semantic Import (SI) model which brought flexibility in transfer of conceptual classes into classification process (Scmidt and Hewitt, 2004), employ fuzziness that maintain indeterminate boundary conditions and express the affiliation of a produced class to ideal landform class (Minar and Evans, 2008).

For the implementation of the proposed method, terrain derivatives (DTMs) that represent local geometry and context at the scale of interest is required at the first place. Classification system that utilize DTMs as input should involve flexible tools to export knowledge base for descriptions into classification, represent continuous nature of terrain classes and indeterminate boundaries via fuzziness, associate spatial entities with interlinkages and assign behaviours to them in order to represent the geomorphometric context and relationships of objects within a hierarchical system. Moreover, per-pixel approach limited by its landscape-independent regular structure is not an efficient way of representing terrain 
having a high spatial autocorrelation. The classification based on attribute similarity of pixels alone, ignoring position is inadequate especially in representing landforms. The minimisation of 'within class' and maximisation of 'between class' differences at pixel domain do not relate to individual landform elements but the whole scene statistically, which is likely to end up with spatially scattered classes across the map.

Current GIS software is not sufficient to fully support complete set of tools to implement those functions. Therefore, this study utilizes an Object-based classification method. Object-based Image Analysis (OBIA) combining powerful characteristics to segment images into objects and building relationships between them which is highly recommended for landscape studies (Blaschke and Strobl, 2001; Drăguț and Blaschke, 2006) is utilized for this study.

Method applies same set of rules with same parameters and thresholds to different case areas with different characteristic and consistent outputs are intended to be gathered. Presenting definitions and parameters used in the proposed method in an explicit manner is also intended since it is believed that explicit criteria would yield results that are more understandable, reproducible and interoperable and adjustable if needed to be adapted for specific purposes.

Study is implemented at a scale that corresponds to a range in between microrelief to mesorelief as defined by Dikau (1989) and it pertains to a scale range coarser than hillslope scale as defined by surface hydrologists, and it is likely to represent landscape scale as defined by ecologist where the majority of land surface processes that has significance in earth sciences take place.

Proposed method is applied on three case areas of $15 \times 15 \mathrm{~km}$ with divergent characteristics of terrain form and complexity.

\subsection{Proposed Outcomes}

Although there have been attempts to materialize the concept of general geomorphometry with general types of landform classifications, they usually have a loose association with a specific discipline like, geomorphology or soil-landscape studies. Classification scheme suggested by Dikau (1989) is quite appropriate to make a general type of landform 
classification, as it exhaustively and generically represents all significant landforms. Proposed classification ends up with a total of 15 landforms namely; nose, spur, spur foot, Shoulder slope, planar slope, foot slope, hollow shoulder, hollow, hollow foot, peak, ridge, saddle, plain, channel, pit. Those classes are not only descriptions of forms but also inherit a higher level context of particular organisation of terrain through relative position and terrain network information.

Study is implemented for a specific scale range that is in between microrelief and mesorelief and it represents landscape scale which is known to be a scale range where most of the significant environmental processes take place.

Classification output can be associated with specific phenomena by relevant discipline, can be further reclassified as landform associations and integrated in nested hierarchies (Minar and Evans 2008), it can be used as a base map for field sampling studies, it can just be used as a general map that characterizes the terrain, or it may even be used as a terrain information map in daily life to serve people; e.g. tourists, as the class names are generic and they are commonsense to people who are outside discipline perception of earth sciences.

\subsection{Method of the Study}

This study is entirely implemented on computer environment with main part in GIS including various GIS software. Method implementation can be thought of as two parts; first part involves preparation of data set, and second part, the object-based classification.

First part (data preparation): Various GIS software have been used for the production of inputs. DEM is produced with Topogrid utility in ArcGIS tool box which is an implementation of ANUDEM algorithm. DTMs were generated out of this DEM. Morphometric DTMs; e.g. slope, plan curvature, etc. were mainly produced in Landserf 2.3 - a freeware by Jo Wood (Wood, 1996)which enables use of user-defined window size for various derivatives calculation at particular scales. Relative terrain position is calculated with TPI which is an ArcView extension developed by Jenness Enterprices (Jenness, 2006). Terrain network is extracted using TNTmips of MicroImages that utilizes an implementation of multi-flow algorithm. There is loose coupling in between all these software and some of them lack standard GIS functions, therefore derived DTMs were imported, analysed and stored in TNTmips and ArcMap. 
Second part (classification): An object-based classification is implemented. For the fulfilment of the proposed method that is object-based rather than pixel-based, an objectbased analysis system that is capable of incorporating semantic import model in class definitions to produce fuzzy classes, and that can build interlinkages within and between objects in a class hierarchy is required. There are OBIA tools rather embedded as tool boxes in recent releases of some GIS software and there are also some limited free wares, however none of those are comprehensive and provide full set of OBIA functionalities required. The commercial OBIA software that is fully capable of implementing proposed method to date is eCognition of Definiens Inc. This software has both evaluation and professional releases, where evaluation release have all the analysis tools but is limited in saving outputs and rules and exporting results to another environment which is also necessary for this study. After a request from my side, a Professional version of the software is allocated for the implementation of this work by Definiens Team, as scientific short term licensing. Classification part and further transfer of the results into other GIS environment is implemented in eCognition Developer Pro v. 8 of Definiens Inc.

Research visits have been made to Z-GIS (Zentrum Für Geoinformatics) Salzburg University which is one of the five 'academic centre of excellence' of Definiens across the world (Definiens Partner Evolve Program, 2010). A workspace with Definiens Professional was provided during these periods and besides chances to attend workshops and seminars on Definiens and OBIA and many occasions to discuss with people on landform, landscape and OBIA.

Some geographers and geomorphologists volunteered evaluation of the results. A reference dataset is provided to them via email to be returned as a list of landforms assigned to random point locations.

\subsection{Organization of thesis}

The thesis includes eight chapters that cover corresponding subjects in an organized manner. A brief description of each chapter is as follows:

Chapter 1 introduces the reasons that motivated me for the proposal of this thesis and describes what is proposed, what the scope of the thesis is and what the intended outcomes are. Method of the study including basic tools that are implemented for the study is 
described. Finally it includes information about what will be included in the proceeding chapters.

Chapter 2 gives background information about theoretical basis on landform classification, characteristics of landforms, literature review that covers trends in landform classification. Scale is of significant importance for landform classification studies therefore a section is devoted to scale concept in landform classification. DTMs that are utilized as input in this study and algorithms that are used to derive them are briefly explained and finally, Objectbased Image Analysis which deserves a brief introduction takes place.

Chapter 3 covers description of proposed method; classification basics, data set used and the classification method.

Chapter 4 describes the case areas where the classification is applied.

Chapter 5 covers description of the methods to derive input dataset being used in the analysis.

Chapter 6 describes the steps of object-based analysis and classification of landforms

Chapter 7 involves evaluation and discussions both on the resultant outcomes and on the method.

Chapter 8 involves conclusions. 


\section{CHAPTER 2}

\section{BACKGROUND}

\subsection{Landform Classification}

Landform classification is basically reducing terrain complexity into a limited number of easily discernible functional units that carry useful information about terrain (Burrough et al., 2000). Landform classification, like any other categorization attempt by human is intrinsic. It is more likely that we can understand what the categories of land reveal, than to perceiving and evaluating continuous representations. Moreover there is a long tradition of mapping, which can be attributed to the relative ease of representing discrete spatial units compared to understanding and evaluating continuous representations of surface (Strobl, 2007). Landform classification emerged due to complexity of the earth surface which has necessitated seeking methods to quantify its form and subdivide it into more manageable components (Evans, 1990) or simply as imperfect generalizations of the reality needed in daily life (Dragut and Blaschke, 2009).

The shape of terrain, i.e. landforms, influence the flow of surface water, transport of sediments, soil production, and determines climate on local and regional scales, furthermore, natural phenomena like vegetation are directly influenced by landform patterns and their relative position across the landscape (Blaszczynski 1997; Coops et al. 1998; Blaschke and Strobl 2003).

Therefore, landform classification has been of great interest in earth sciences and neighbouring disciplines. Ever since landforms could be delineated from maps and other sources (Cayley, 1859) there have been vast amount practice on landforms from various area of interest. Landforms although have different meaning to different disciplines, they reveal common physiological and morphological characteristics of terrain which may guide through understanding past and present processes acting on terrain and provide necessary 
information to related disciplines about land characteristics and potentials. Landform classification has a wide range of application domains including mapping geomorphologic units (e.g Asselen and Seijmonsbergen, 2006), mapping lithology (e.g. Kühni and Pfiffner, 2001) predicting soil properties (e.g. Gessler et al., 1995; McKenzie and Ryan, 1999; Florinsky et al. 2002), landscape ecology (e.g. Ford et al., 1997), vegetation mapping (e.g. Franklin, 2000; Pfeffer et al., 2003), precision agriculture (Verhagen et al., 1995), landslide mapping (e.g. Carrara et al., 1991).

Landform classification indeed constitutes a central research topic in Geomorphometry; science of quantitative description and analysis of land surface (Pike, 2002; Rasemann et al., 2004)

Geomorphometry is usually referred to as a sub discipline of Geomorphology (Dehn et al., 2001; Bolongaro-Crevenna et al., 2005), as it has been established under Geomorphology as an interdisciplinary field from mathematics, and Earth sciences and computer science, it is now often regarded as a separate discipline on its own right (Pike et al., 2009). Geomorphometry until 1950s produced poor qualitative descriptions of landforms due to lack of quantitative methods (Sinha, 2008) with the advent of computer technology, analytical techniques, digital representations of the surface, GIS and image processing tools, Geomorphometry has evolved into a new scientific concept (Pike, 2002). Hence it is more recently described as the science of terrain quantification focused on extraction of landsurface parameters and objects from Digital Elevation Models (DEMs) (Pike et al., 2009).

Landform classification is a central research topic in geomorphometry and geomorphology that seeks form-process relation at its core and effort for attributing physical and functional meanings to land surface considering past processes (Evans., 1998). However beyond this focus rooted in process, landform classification provides important information to other disciplines which are involved in "geo" issues either fully or partially. Main reason for utilizing landforms as basis or supplementary information into their work is that landforms exhibit common physiological and morphological characteristics which might serve as a boundary condition in their application. Moreover, landform units inherently substitute an important information gap on land characteristics and potentials. Rather than getting through difficulties of measuring the biophysical environment that is to a very certain extent controlled by variation in topography, landform boundaries or terrain unit boundaries are utilized (Deng et al., 2006). 
Early attempts to classify landforms - actually term "delineate" is more proper than to "classify" was subject to expert bias and a tedious task to implement since it heavily relied on manual delineation of landform units based on visual interpretation of stereo aerial photographs or field survey (Burrough et al. 2000).

Conventional methods of landform delineations based on stereo aerial photo interpretation and field observations has evolved from those manual methods to computer-based techniques that rely on Digital elevation Models (DEM) and Digital Terrain Models (DTMs) of various scales, sources, and kinds. Since computer-power, GIS tools and a vast amount of digital elevation data became easily accessible, much more research topics and application area has emerged in geomorphometry and neighbouring fields focusing on landform classification (Dehn et al., 2001).

\subsubsection{Brief History}

Mapping landforms, particularly systematic measurement of topography from cartographic sources can be dated back at least to mid-nineteenth century (Cayley, 1859; Maxwell, 1870). The accessibility of computing technology in the 1970's was innovative in the research interest, particularly with the work of Evans $(1972,1980)$ and Mark (1975).

More recently, the advent of Geographic Information Systems (GIS) accompanied by the widespread availability of digital representations of terrain broke new ground in landform mapping (Wood, 1996). Mutually there has been an interest on quantification of the surface measurements and automating the delineation task.

Landform mapping for a long period was based on visual interpretation of stereo aerial photographs and topographic maps supported with field surveys and geomorphometric properties have been measured manually for decades (e.g. Horton, 1945; Miller, 1953). Visual interpretation and delineation of the landforms is tedious and measurement of parameters from topographic maps is labour intensive and time consuming. As a consequence those mapping efforts were restricted to areas of limited extent. Moreover, those conventional landform classification efforts were based on implicit rules which are often subject to expert bias (McBratney et al., 1992). They relied heavily on the expert's experience and conceptual model in mind (McKenzie et al., 2000) which is being materialized implicitly on to maps. There is considerable empirical evidence in the literature 
where different surveyors delineate a complex landscape in a variety of ways, according to their personal insights (e.g McBratney, 1992; VanWesten, 1993).

One of the first geomorphometric-based delineation of landforms dates back to Penck (1894). His model was based on the assumption that all landforms can be assigned to a set of some fundamental type. Simple form elements represented the lowest level and higherlevel landforms were created by aggregation of lower-level landforms. Furthermore, Penck (1894) suggested fundamental landform unit as the "form element" and it was defined by its curvature. Although parameters and their manual measurement methods were not exactly defined, these approaches were representatives of the manual delineation of landforms out of conventional sources.

The earlier attempts to convert manual and expert-driven routines into more explicit and straightforward methodologies emerged even before computers come out. Seminal work by Hammond (1964) represents the earliest of those approaches and it was practically applied to classify landform types for whole US by USGS (Hammond, 1970) and required several years to complete. Hammond's procedures for applying the classification rules were manual, primarily through visual analysis of 1:250.000-scale topographic contour maps. Terrain was characterized within a local window $(9.7 \mathrm{~km} 9.7 \mathrm{~km})$ that was moved without overlap across the contour maps. Within each window, he quantified the percent of the gently sloping topography ( $<8 \%$ slope gradient) and the difference between the lowest and highest elevation points (relief) within the window. Those values were then used to quantitatively identify different landforms with a look-up-table to yield a coarse resolution continental landform map. Hammond's method was primitive and had many shortcomings, even notified by himself (Hammond, 1964); i.e. profile type was not taken into account. This method has been applied very broadly in the U.S and it has pioneered other studies; his approach has been exercised afterwards by various authors, first automated by Dikau et al. (1991) and was modified by Brabyn (1998) and by Gallant et al., (2005).

Progress in automating the theoretical concepts in landform mapping has two significant breaks; first is the advent of computers and the second is the advent of GIS. Starting with the advent of computers by 1950s and availability of Digital Elevation Models (DEMs) and derived data sets, such as slope, aspect, hydrographical pattern and shaded relief have been exploited to examine terrain with automated or semi-automated methods (Evans, 1972; Mark, 1975; Carrara et al., 1977). Development of computer technology, advent of digital data sources and transfer of quantitative analysis and display techniques into computer 
programs provided a primitive environment that enabled the application of numerical techniques for terrain analysis and landform classification (Schmidt-McGibbon,1993).

However, it was the advent of GIS technology and digital image processing techniques that brought significant advances in terrain parameterisation (e.g. Evans, 1980; Zevenbergen and Thorne, 1987; Moore et al., 1991; Wood, 1996; Florinsky, 1998; Wilson and Gallant, 2000). They proved to be crucial to later developments of automated landform classification (e.g. Irvin et al., 1997; Burrough et al., 2000; MacMillan et al, 2004, Schmidt and Hewitt, 2004); particularly after Pike (1988) introduced the concept of describing a landform by geometric signatures. For about two decades, quantitative geomorphometry or digital terrain analysis (Pike, 2002) which also include landform classification with the use of DTMs as input established a formal area of research.

In consequence, challenges associated with terrain modelling, quantification and automated classification of landforms has become easier, yet the whole concept became more sophisticated, as new constraints and concepts were introduced. Automated computer-based procedures require explicit criteria and assumptions that forced experts to systematically encode and quantify their knowledge (MacMillan et al., 2004). Favourably, that led to testable and reproducible results, increased consistency and reliability of the products and enhanced interoperability.

\subsubsection{Classification Approaches}

Increasing demand for producing or adopting landform schemes, various applications and research directions on landform classification has lead to very heterogeneous views and definitions of landforms and there is a vast amount of methods to achieve landforms.

This heterogeneity can be attributed to diversity in research and application domains. Each discipline has its own motivations and reasons to produce or adopt a landform classification. Therefore landform classification produced by a particular discipline perception might be irrelevant for use of another discipline.

Main difference in view points obviously is that; landform possesses at least two important properties: (i) landform is a result of past geomorphic and geologic processes, and (ii) landform is a controlling boundary condition on actual geomorphologic processes and other 
phenomena like microclimate, ecology, surface hydrology, etc.(Dehn et al., 2001) These two characteristics lead to different points of view of landform, where former is focused in static boundary conditions to seek for the reasons that shape the topography, while the latter is interested in the consequences brought by those boundaries. Even under these this two mainstreams there are subsets that pose vast amount of divergence in terrain conceptualization associated with different motivations to produce landform maps relevant to their research topic and application domain. Especially for environmental sciences like landscape ecology, vegetation studies, etc. classifying the terrain into homogeneous classes provides combined representation of spatial and statistical data (Longley et al., 2001) which reduces the complexity of the landscape, where compound topographic indices are often used to classify terrain into landscape units or ecotones or ecoregions rather than landforms.

Evans (1972) who was one of the first to point out this diversity in terrain studies and lack of interoperability in landform classification grouped attempts to classify terrain form as; 'general geomorphometry' and 'specific geomorphometry'. "Specific geomorphometry" is restricted to particular concepts, it is more strict and it is on purpose. "General Geomorphometry" on the other side involves the quantitative analysis of characteristics of terrain, which are applicable to any continuous rough surface - even outside geomorphology. Features described in general geomorphometry can be thought of as morphometric rather than geomorphometric in that they are characteristic of any surface (Wood, 1996). For a 'general' type of classification where classes are not described by specific form and process, a classification scheme which contains a set of appropriate morphometric / geomorphometric classes should be defined.

This distinction by Evans (1972) of the terrain analysis placing it into one of the specific or general geomorphometry provides a framework for classifying morphometric parameterization, so that various approaches to landform classification fall into one of these broad categories eventually. An equivalent discrimination was made by Dehn et al. (2001) who described geographic objects in two ways, i) based on their geometry or ii) based on their semantics. Term 'semantics' in the latter approach imply; objects are named regarding a model in mind or specific background relevant to a discipline perception.

\subsubsection{Classification based on Specific Geomorphometry}

Specific geomorphometry involves detection and delimitation of discrete features like, cirques, drumlins, sand dunes, alluvial fans, landslides, dolines (Pike et al., 2009). 
Delimitation of those specific geomorphologic features requires operational definitions and precise thresholds of parameters to ensure their proper characterization (Evans and Cox, 1974).

Specific geomorphometry also relate to domain applications such as soil-landscape modelling, surface hydrology, ecological mapping, climate studies, since these fields extract specific landforms relevant to their interest using terrain parameters. These attempts like most of the other classification attempts are on specific purpose and wide range of application domains that relate to specific geomorphometry covers a very big part of the landform classification studies.

\subsubsection{Classification based on General Geomorphometry}

General geomorphometry involves quantitative characterization of any surface. It provides a basis for the quantitative comparison of different landscapes and it can adapt methods of surface analysis used outside geomorphology (Pike et al., 2009). Specific geomorphometry has a much longer history, starting with measurements of lunar craters (Schloss, Milton, $1965)$ as specific geomorphometry pertained to earth surface land and more general definitions were needed. Today's general geomorphometry is particularly different than the former. Bbecause conventional general geomorphometry was extremely difficult before the advent of computers: today with the processing capabilities and large availability of DEMs it has become widespread and has many applications in digital terrain modelling (Pike 2000). Especially general geomorphometry in quantification of global/local measures of the surface are very common, e.g hypsometric curves (global), curvature of a surface (local). Landform classification based on general geomorphometry deals with elementary forms and is less common compared to wide range of application domains that relate to specific geomorphometry (Pike et al. 2009).

General geomorphometry is relatively universal; it avoids problems of landform subjective definition to an extent. Subjectivity and operator bias are reduced drastically, though not totally eliminated (Evans, 1990). It appears objective, but it involves choice of data source, resolution, and algorithms for interpolation, smoothing and derivative calculation. One important characteristic and also drawback of landform classification based on general geomorphometry is that it lacks in precision where, specific geomorphometry is precise, but can only be applied in a specific context (Wood, 1996). Even these two aspects of geomorphometry were supposed to two polarized ends (Wood, 1996) they are not 
completely distinct, first because some specific landforms such as hill slopes and drainage networks are so widespread on Earth that their specific geomorphometry acquires a general importance: and second, because some techniques of general geomorphometry can be applied to specific landforms (Evans 1987).

\subsubsection{Automated Landform Classification}

Terrain surface exists as spatial continuum (Deng and Wilson, 2008), and surface form varies considerably moving across landscape. Earth scientists for decades have exercised methods to reduce this totality of surface spatial variation to a limited number of easily discernible functional units that may carry useful information about surface hydrology, soil properties or site stability (Burrough, 2000).

Many authors (Pike 1988, Dikau, 1989; Iwahashi,1992, Moore et al., 1993) at the early stages of incorporation of GIS into landform classification studies have noticed that DTMs generated out of DEMs through certain image-processing operations appeared to reproduce terrain form and context efficiently. The resemblance suggested that satisfactory landform maps might be created rapidly and entirely from DEMs, and also that the bias of each airphoto analyst's "personal equation" could be eliminated. The multiple criteria for terrain classification emerged from extended trial-and-error experiments with different DEMs and DTMs produced via various algorithms in conjunction with geometric interpretations of terrain (Iwahashi and Pike, 2007).

As opposed to conventional landform classification methods, automated landform classification based on numerical data is pragmatic, repeatable, robust (Ventura and Irvin, 2000; Asselen and Seijmonsbergen, 2006), and interoperable (Longley et al., 2005) although not completely eliminating subjectivity (Evans, 1990). A number of automated methods and procedures for landform classification have been developed as geomorphometry has increasingly overlapped with the domain of GIS (Evans, 1998). Although they differ in methodology and the variables that they utilize, what they have in common is that they use DTMs derived from a DEM as input.

Previous attempts to devise automated methods of landscape classification out of DTMs have been frustrated by computational issues related to the size of the data set (Burrough et al., 2000). Recently, along with the development of GIS; Digital Terrain Analysis 
techniques and computation capabilities, processing very fine resolution multivariate datasets became very efficient and the study of automated landform classification has been enhanced. As a consequence, landform classification turned out to be a formal research topic evolved from computer technology and traditional geomorphometry.

\subsubsection{Trends in automated landform classification}

Landform classification has started on the same course as classification of satellite images. DEMs and DTMs are very similar to remotely sensed images in the way they store data i.e. regular gridded raster with DEM/DTM values instead of spectral reflectance values. Landform classification is inspired by the conventional image classification techniques used in remote sensing. e.g. DTMs were put through multivariate statistical analysis like ISODATA or k-means seeking clusters in the data cloud (e.g. Irvin et al., 1997) or statistical proximity e.g. Euclidian distance to a given training set to yield a list of predefined classes (e.g. Prima et al., 2006).

Those classifications usually yield crisp boundaries like in conventional thematic maps as they were practically intended to replace or update old thematic maps. Although the data storage is very similar, the phenomena they represent is quite different, where satellite images have spectral reflectance values of features on earth surface, and DTMs store variability of continuous land surface. As a consequence, landform classification is likely to proceed from the traditional approaches to analysis towards a more stable semanticsoriented definition of terrain units (e.g. MacMillan, 2000; Schmidt and Hewitt; 2004), from crisp classifications to fuzzy or continuous classifications (Irvin et al., 1997, Burrough et al., 2000) that better represents continuous nature of landform classes, from pixel based approaches to object-based approaches (e.g. Dragut and Blaschke, 2006; Asselen and Seijmonsbergen, 2006). Object-based Image Analysis (OBIA) which was primarily established for remote sensing practices is proved to be efficiently adopted in terrain classification applications. Following section describes those general trends in landform classification research.

\subsubsection{Semantically oriented vs. data-driven classification}

The main alternatives to classify landforms given a set of DTMs are: (i) to use statistically based data-driven techniques (e.g. cluster analysis) or (ii) to utilize predetermined semantic 
descriptions or expert knowledge of morphometric characteristics of each landform (MacMillan et al., 2000), also referred to as deductive/inductive classification (Arrell, 2007) or supervised/unsupervised classification (Prima, 2006).

Data-driven landform classification can be performed without a priori knowledge on the classes, and class centroids are iteratively detected using clustering algorithms given the number of classes. Many researchers have employed clustering algorithms with a variety of morphometric derivatives as input into an automated morphometric classification of landforms (Irvin et al., 1997; de Bruin and Stein, 1998; Burrough et al., 2000; Macmillan et al., 2000; Ventura and Irvin, 2000). Classification procedures based solely on statistical clustering can identify the optimum number of "natural" classes for a given site and classes gathered are statistical classes rather than information classes that are optimized iteratively to exhibit minimum differences within and maximum differences amongst each other.

Well-known study of Irvin et al. (1997) is an application of data-driven fuzzy classification on DEM, slope, aspect, profile curvature and plan curvature to automate landform classification. Study aimed at producing a soil map where landform information constitutes a very important component in soil mapping. They hypothetically assumed that DTMs correlate with observed soil properties. DTMs were classified through ISODATA and kmeans and classification yield landforms quite similar to that of manually delineated as they reported.

Clustering algorithms are sensitive to input data and result in classifications that are optimum for a given dataset. Therefore those algorithms can yield considerably different outputs with incompatible classes when applied to another area and hence it is not easy to generalize classification for a wide variety of landscapes. This causes problems in application and reduces model validity.

Most of the authors (MacMillan et al, 2000, Dehn et al., 2001, Schmidt and Hewitt, 2004) suggest adopting a single set of semantic rules based on expert knowledge to overcome this problem. Semantics are the bridge between language and thought, and imply the meaning that is attached to real world objects and concepts (Jackendoff 1983). Pike's (1988) proposal of "geometric signature" transfers knowledge of geomorphometric classes into automated classification. Pike (1988) describes geometric signature as "a set of measures that describes topographic form well enough to distinguish geomorphologically disparate landscapes". Hence, geometric signature can be utilized as a basis for training set that represents the class 
central concept in a supervised image classification. Many studies in landform classification has adopted supervised classification techniques either fuzzy or crisp (e.g. Dikau, 1989; Dikau et al., 1991; Dymond et al., 1995; Brabyn, 1998; Brown et al., 1998; MacMillan et al., 2000 , Prima et al., 2006). If fuzzy; classification is either based on similarity relation model or semantic import models. Similarity relation or semantic import model in supervised classification relate to fuzzy implication of supervised classification and presented in the next section.

\subsubsection{Fuzzy vs. Crisp Classifications}

Classification of multivariate data by means of statistical analysis, either data-driven or semantically oriented is a common practice within the fields of GIS and remote sensing.

The use of such algorithms for classification has proved to be successful when applied to data that represent naturally crisp classes with little overlap in attribute or geographical space. This is often the case for remotely sensed imagery; where the classes that are extracted from such data exist naturally. These classes reflect different types of land-cover that are more or less distinct; any overlap between classes is mainly an issue of scale rather than ambiguity in class definitions (Romstad, 2001).

DTMs however depict a multivariate continuum that has little or no distinct class boundaries because there is an inherent overlap between different classes, in both spatial and attribute space (Burrough and Frank, 1996, Fisher et al., 2004; Romstad, 2001). With the exception of some on purpose landform classification tasks where rigid and explicit definitions can be made on classes (Graff and Usery, 1993; Prosser et al., 1997), high degree of uncertainty in the semantic descriptions of land elements makes a unique and unambiguous classification into land elements hardly possible (Schmidt and Hewitt, 2004). If landform classes are separated by crisp, unambiguous boundaries, the attributes of landscape that vary gradually over space are represented by sharp transitions that may result in a loss of useful information plus an increase in error due to the arbitrary placing of inappropriate boundary lines (Heuvelink and Burrough, 1993).

The theory of fuzzy sets (Zadeh, 1965; Robinson, 1988) provided a possible solution to the 'crispness' problem in traditional landform classifications. Fuzzy classification - also referred to as 'continuous classification' that take the natural class overlap into account allow individual spatial entities to have partial belongings (memberships) to multiple 
classes, and therefore allow existence of overlapped classes that have gradual transition from one another. The degree to which an individual belongs to a given class is not expressed in terms of a Boolean logic ( 0 or 1$)$, but fuzzy logic (a continuous membership value that ranges in 0 to 1 ). This means that they are suitable in situations where there is similarity between an individual and a class, rather than exclusive membership (Burrough, 2000). Methods of fuzzy classification are replacing crisp methods of multivariate classification because they allow class overlap which is very common for natural groups in the terrain phenomena.

There are two methods of defining the membership values of the fuzzy sets; 'similarity relation model' and 'semantic import model' (Robinson, 1988; MacMillan et al., 2000; Fisher, 2004). Similarity relation model uses surface derivatives as input to a multivariate fuzzy classification via a training set to define its 'class central concepts' and yields the class membership values for each case i.e. raster cell (Brabyn, 1998; Burrough et al 2000; MacMillan et al.2000; Prima et al. 2006). Method requires central concept to be defined by means of a training set, then image elements (pixels or objects) are classified in to one of the classes based on attribute similarity given the class central concept. However this is not always a very flexible way of translating the conceptual model in mind.

Semantic Import (SI) model however permits formal recognition and incorporation of the imprecise and overlapping semantics used to describe or classify data (MacMillan et al. , 2000). SI model is useful in situations where users have a good, qualitative idea of how to group data and membership function should ensure that he grade of membership is 1 at centre offset (Burrough, 1989; Matternicht, 1999). In the case of terrain classification, the SI model permits experts to identify conceptual landform elements and to make class definitions using imprecise semantics (Cheng and Molenaar, 1999; MacMillan et al., 2000; Dehn et al., 2001; Schmidt and Hewitt, 2004). User interaction and weaknesses coming from translation of class descriptions i.e. definition of thresholds or shape of the SI curve is still of concern.

\subsubsection{Object-based vs Pixel-based Approaches}

DEMs and DTMs that are derived from DEMs, can give us valuable information on the characteristics of landforms. Each pixel on the regular grid has a value of the approximated measurement of specific terrain attribute; e.g. slope, curvature and these pixels can be categorized into classes regarding statistical clusters or similarity to class centroids. A 
problem, however is that, features on the land surface are poorly represented with individual cells which have dimensions that are regular and independent of the landscape character. Pixels are not geographical objects and pixel topology is limited in representing particular organization of landscape (Hay and Castilla, 2006). Moreover, current pixel-based image classification largely neglects spatial autocorrelation that significantly exist in terrain data (Strobl, 2007). Hence, spatial aspect, which is very essential in terrain context, can be only poorly represented in a pixel-based domain (Romstad, 2001).

Features that are larger than the pixel size require a higher level of context for accurate identification in a DTM grid (Giles, 1998). Higher level context in the search for landform features can incorporate feature boundaries using contextual clues (Mackay et al., 1992).

Mackay et al. (1992) were one of the earliest who advocated use of higher-order geographic objects rather than pixels for topographic characterization, and incorporated fuzziness to represent indeterminate boundary delineations. They generated objects called HOGO (Higher-Order Geographic Objects) to represent topographically homogeneous entities.

Landscape ecology theoretically involves the similar concept of homogeneous entities. Landscape element or patch is agreed upon as the smallest unit in the landscape (Forman, 1995; Wiens, 1997). Ecotope, biotope, habitat, site, (Forman and Godron, 1986) all can be referred to as "patch' or "landscape element" that motivated many researchers to utilize object-based domain in landscape studies (Blaschke and Strobl, 2001; Burnett and Blaschke 2003; Mallinis et al., 2008)

"Objects" in the context of image analysis can be defined as groups of grid cells of a raster data, created from a segmentation process. They contain far more information than individual cells addition to spectral information from the cells (Asselen and Seijmonsbergen, 2006). They carry information about their e.g., shape, size, adjacency, enclosure, proximity to other objects Landform segmentation thus provides a basis for further classification with many capabilities specific to object-based image analysis.

Advent of image segmentation dates back to mid 1970s (Haralick et al., 1973), however it became available in software packages and studied as a standard image analysis procedure for more than a decade only. Object-based Image Analysis (OBIA) was primarily established for remote sensing practices (Dragut and Blaschke, 2006). Studies have shown that object-based classification is effective for the analyses of digital elevation data (Hoffmann and Vegt, 2001), and it is being explored in terrain classification applications 
(e.g. Miliaresis and Argialas; 1999; MacMillan et al., 2000; Miliaresis, 2001; Strobl, 2001; Blaschke and Strobl, 2003; Drăguț and Blaschke, 2006).

For instance; Dragut and Blaschke (2006) reported promising results on their object-based classification of landforms. They employed multi-scale image segmentation algorithm in combination with fuzzy classification to automatically extract landform elements for two contrasting terrains. In their segmentation algorithm, the 'scale parameter' was defined as the maximum change in the total heterogeneity that may occur when merging two image objects. They derived some of the classes that correspond to 'form element' model of Dikau (1989) with their classification built both on the surface shape and on the altitudinal position of objects. They employed slope, plan curvature, profile curvature and relative altitude as input into their classification.

\subsubsection{Other Techniques}

Advanced techniques in image classification like Artificial Neural Networks, Support Vector Machine, and Genetic Algorithms are also being evaluated in landform classification research. Artificial Neural Networks (ANN) can handle non-convex decisions. The use of textural features helps to resolve misclassification that is very common in pixel-based classification of landforms. The support vector machine (SVM) is a group of theoretically superior machine learning algorithms. It was found competitive with the best available machine learning algorithms in classifying high-dimensional data sets, e.g. SVM for a test site on Mars, produced the most accurate results compared to other conventional techniques for classification of topographic objects (Stepinski et al., 2006). The genetic algorithm searches a space of image processing operations for a set that can produce suitable feature planes, and a more conventional classifier which uses those feature planes to output a final classification. ANN employs learning algorithms to supervise classification and it is applied in landform classification studies usually with specific purposes (e.g. Hosakawa et al., 2002)

\subsection{Landforms}

Terrain is a continuous phenomenon structured into landforms as a result of the cumulative influence of geomorphological, geological, hydrological, ecological, and soil forming processes that have acted on it over time (MacMillan and Shary, 2009). Landforms are therefore widely recognised as natural objects that partition the Earth's surface into 
fundamental spatial entities. Landform entities differ from one another in terms of characteristics such as shape, size, orientation, relief and contextual position. They also differ in terms of the physical processes that were involved in their formation and that continue to operate within them at the present time (Etzelmüller and Sulebak, 2000).

All major approaches of terrain classification treat land surface as composed of smaller units that are usually called landform elements or units. Operations that are carried out on the landform are delimitation of units following a set of definitions regarding homogeneity of form or structural constraints and/or aggregation of those units into larger forms or objects (Dehn et al., 2001).

There are many definitions of landform from different discipline perceptions, which causes heterogeneity in definition and labelling of landforms. Next section is devoted to some of those definitions on landforms suggested in the literature.

\subsubsection{Landform definitions}

According to Bishr (1998) objects and their interrelations forms a mental model which is part of the 'discipline perception world', hence 'semantics' are strongly dependent on the mental model i.e. discipline itself (Dehn et al., 2001). This means that objects are labelled in line with specific background or within the context of a particular discipline. Here are some of the definitions on landforms from different perspectives:

A subjective semantic definition consistent with 'specific geomorphometry' describes landform as "a terrain unit created by natural processes in such a way that it may be recognised and described in terms of typical attributes where ever it may occur" (Hammond, 1965). A landform unit that fits to this description should have a strong formprocess relationship; hence it is most probably a cirque, alluvial fan, etc.

From a physical geographers point of view (Whittow, 1984) defined landform is 'the morphology and character of a land surface that results from the interaction of physical processes upon the surface material'. 
Hammer (1997) who is a soil scientist suggest that a landform is defined not only by its surface shape, but also its location in relation to other landforms, its underlying geologic materials, and its soil attributes.

Pike et al., (2009) described landforms as division of the land surface, at a given scale or spatial resolution, bounded by topographic discontinuities and having (relatively) uniform morphometry.

There is a very broad description of landform in 'Glossary of Geology' (Bates and Jackson, 2005). Accordingly, a landform is defined as "any physical feature of the Earth's surface having a characteristic, recognisable shape". This definition of landform is generic and relates to human cognition of landforms; i.e. landform is recognisable.

As humans in our daily lives have labels for recognizable shapes of the terrain like mountain, hill slope, peak, ridge, etc. and those classes address more or less the same landform. It is noted "more or less" as everybody is using terms in different ways, especially if they are translated into another language, e.g. what is called a 'Berg' in German may not coincide perfectly with what is called a 'mountain' in English (Smith and Mark, 2003). Mark and Turk (2003) for instance have found fundamental differences in descriptions of landforms between a native Australian language and English; hence it is very likely that we have a degree of heterogeneity in landform nomenclature. A name of the class for instance may refer to two forms which do not perfectly overlap or a particular landform may be entitled with different names. This heterogeneity in nomenclature however is only part of the problem. Perception worlds lead to a cognitive semantic heterogeneity which is situated deeper in the perception of reality and is difficult to address (Dehn et al., 2001).

Heterogeneity in definition of landforms is basically related to semantics. 'Semantics are the bridge between language and thought, and imply the meaning that is attached to real world objects and concepts' (Jackendoff 1983). Ontology has been discussed in GIS as a standardization procedure through which easier translation between different information sources can be achieved (Fonseca et al. 2002).

In the next section there is brief explanatory information on landform ontology. Actually, even landform itself due to its indeterminate characteristics and vagueness (Fisher et al., 2004) constitutes a formal discussion topic in ontology. 


\subsubsection{Landform Ontology}

Ontology deals with the existence of all kinds of entities, abstract and concrete, that make up the world (Sowa 2000). In the Oxford English Dictionary (OED), ontology is defined as the 'science or study of being' (OED, 2010) and on Wikipedia (2010) it is defined as the philosophical study of the nature of being, existence or reality in general, as well as the basic categories of being and their relations. As a part of the major branch of philosophy known as metaphysics, ontology deals with questions concerning what entities exist or can be said to exist, and how such entities can be grouped, related within a hierarchy, and subdivided according to similarities and differences.

Landform as components of a continuous land surface which has connections to human thought and action are largely discussed and evaluated in ontology. 'Mountain' is for instance very commonly used metaphor in ontology; Discussion on ontology of mountains represents necessary information on ontology of landforms as well.

'Mountain' is supposed to be 'vague' in a philosophical sense (Sainsbury 1989; Williamson 1994; Fisher et al., 2004), which means; either the boundary condition is not actually distinct, or the class, which describes a site may vary with perception where those ambiguities are being managed with the incorporation of fuzzy classification methods (Gale, 1972; Robinson, 1988; Fisher, 2004).

Smith and Mark (2003) in their seminal paper titled 'Do mountains exist?' are discussing about the ontology of mountain as geographic feature. This type of questions seeking the answer of 'does it exist?', “is this real” or specifications of 'what constitutes reality' forms the basis of ontology described from a pure philosophical sense (Smith 1995).

The term 'ontology' is formally used by information scientists, in a sense distinct from that disseminated by philosophers. It is not concerned with questions of existence; rather, it is designed to assist in the task of specifying and clarifying the concepts employed in given domains and formalizing them within the framework of some syntactic and semantic structure, this variety of ontology is concerned with questions such as: 'Should mountain be a category in our knowledge base?', 'if so, how the category mountain should be defined?' and 'how individual mountains should be represented in a database?' (Smith and Mark, 2003). Accordingly; mountain is the label of a geographic feature that is cited by English 
speakers and it clearly exist in human thought and action. However, as Smith and Mark (2003) suggest a mountain does not exist in the way that a 'cat' exist independent of a humans citation, and there is no determinate boundary that separates a mountain from an adjacent geographic feature as sharp as the boundary that cat is separated form the carpet which is a real object as well. The explanation lies in the ontology; hence closely related to categorization in cognitive science; Mountains are not 'real' but they are 'fiat' objects.

Deng et al. (2007) in their review paper provide ontological evaluation of recent progress in terrain analysis and outlined categories of landforms in the way they exist in human knowledge. Accordingly; they categorized landforms into five ontological types, where three of them will be mentioned here, as the other two is thought of as versions of these three;

Bona fide/real landform objects: Bona fide landform objects are 'real' or of 'natural kinds' because they have the least dependency on human definition (Plewe, 1997). The term 'natural kind' has been defined as: 'the objective reality investigated by science is populated by mind-independent natural kinds of things, which are characterized by the fundamental, intrinsic causal powers which they possess' by Sankey (1997).

A watershed for instance, is a real object and is surrounded by real, crisp boundaries beyond which surface runoff would contribute to a different outlet (Graham et al., 1999; Band et al., 2000). It is therefore surface/subsurface runoff processes that determine the thematic meaning and geographic delineation of watersheds and boundaries. Prominent summits may also be treated as bona fide because their existence as distinguishable highest points is unquestionable.

Semantic/Fiat landform objects: Semantic or fiat landform objects do not have absolute references as bona fide objects or prototype objects. They rely almost entirely on human definition and there is usually more than one way of defining a fiat object (Dehn et al., 2001; Smith and Mark, 2003) such as a steep slope vs. gentle slope. As a result, delimitation of thematic and geographic boundaries of fiat objects is strongly subjective (Fisher, 2000; Dehn et al., 2001).

Prototypical objects: Prototype is a mode of graded categorization, where some members of a category are more central than the others. Prototype objects reflect the central tendency of 
features or properties of 'real' entities (Rosch, 1973). For example; for prototype 'bird'; sparrow is more central than a penguin, where both are birds.

According to this brief information on landform ontology it is understood that real objects exist at their own right with their boundary conditions where fiat/semantic objects are subject to a conceptual model in mind, or semantics that are strongly dependent on the scientific discipline itself (Dehn et al., 2001). This subjectivity / objectivity issue is similar to that have been mentioned formerly for 'specific' vs. 'general' geomorphometry in Section 2.1.2, and it seems that epistemological grounds of 'specific geomorphometry' depends on the extent to which landforms can be viewed as 'real' objects. Delimiting an alluvial fan - a real object in the sense that it is characterized by mind-independent natural phenomena and that exist as a separate entity with its specific form across the regular landscape- can be extracted automatically as well, with a set of rules introduced by an expert, is a good example of application in specific geomorphometry.

Deng et al. (2007) affirmed form elements of Dikau (1989) as fiat objects with some exceptions like summit in peak areas or a stream channel in a valley which pose real entities. They claim that no point or component in a fiat object (e.g., in a form element, see Dikau, 1989) can be undeniably more typical. However, Qin et al. (2009) suggest that prototype "shoulder slope" is an example in geomorphology and it can be described as locations with maximum change of slope gradient adjacent to both a ridge and a back slope, hence maximum change of slope which refers to highest profile convexity constitutes central concept for 'shoulder slope' one of the form elements of Dikau (1989). Therefore fuzzy memberships could be calculated for those elements based on SI model, just like Schmidt and Hewitt (2004) did in their study.

Similarly; peaks as areal spatial entities can be categorized as prototypical objects and, summit points as the bona fide objects constitutes the conceptual core, thus represent the extreme characteristics and locations of prototypical objects. Bona fide objects this way help defining membership distributions within prototypical objects (eg, Graff and Usery, 1993; Smith and Mark, 2003; Wood, 1996; Schmidt and Hewitt, 2004).

\subsubsection{Landform Characteristics}

Form or local geometry constitutes the basis of a general type of landform classification and it reveals morphometric classes. However, form elements are neutral and they lack linkages to wider topographic context (Deng, 2007), if the surface is pertaining to a piece of land 
then it needs further concern as it is not neutrally labelled in line with its geometry but geomorphometric context as well. Geomorphometric context to reveal organization of form elements across the landscape should be incorporated in order to justify that form elements correspond to real land surfaces.

\subsubsection{Morphometry}

Land surface morphology determined by local geometry and the concept of elementary forms can express the ideal structure of the lowest hierarchical order of taxonomic complexity of landforms (Minar and Evans, 2008), therefore it is fundamental to geomorphologic mapping and many other application domains in earth sciences. Local geometry of the landscape constitutes the fundamental component of terrain units as it reveals the best surrogate to directly/indirectly portray similarities vs. differences on land where there are no or few abrupt changes. Hence form / local geometry typically constitutes the basis of a system of classification of landforms.

Form; not necessarily pertaining to a land surface but any other surface is effectively represented by means of change in the variation of elevation in its first and second order derivatives; slope and curvature. Among various attributes that can be derived from Digital Elevation Models; 'slope' and 'curvature' are the two indispensable fundamentals of the study of morphometry. Primary morphometric attributes derived from DEMs are slope gradient and slope aspect, usually just denoted as 'slope' and 'aspect'. Secondary attributes are the rate of change of gradient and aspect. Rate of change of slope measures the curvature in downslope direction, and is called 'profile curvature'. The rate of change across-slope is the 'plan curvature'. Primary attributes are measured in degrees $\left(^{0}\right)$ where secondary attributes are measured in degrees of change per meter $\left(\mathrm{m}^{-1}\right)$. Form of a surface can be represented as all possible combinations of categorized slope and curvature values Dikau (1989).

For a 'general' geomorphometric classification the best known schemes belong to Peucker and Douglas (1974); a six class scheme; ridge, peak, pass, channel, pit, planar and scheme of Dikau (1989) with 15 classes which represent all possible combinations of terrain slope and curvature.

Scheme of Peucker and Douglas (1974) (Figure 2.1) is a based on a $3 \times 3$ evaluation of DEM, e.g. if the altitude of the centre of the window is higher compared to other neighbouring 
cells than central pixel is classified as 'peak'. Even this scheme has been adopted by many authors (Evans, 1980; Wood, 1996; Bolongaro-Crevenna et al., 2005), it has some major drawbacks i.e. it characterizes each DEM cell but cannot fully describe a group of more complex forms of actual landforms, even if the window size is enlarged (e.g. Wood, 1996). Scheme simply focuses on specific features; peak, ridge, pass, channel, pit. There is only one class that is allocated to planar elements; 'plane', and sloping elements are largely neglected.

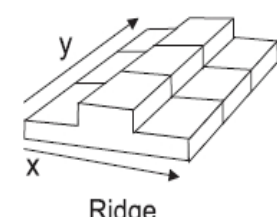

Ridge

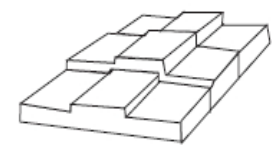

Peak

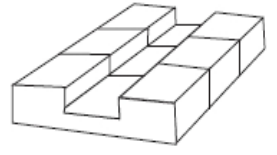

Channel

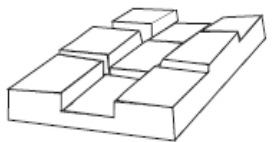

Pass

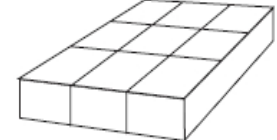

Plane

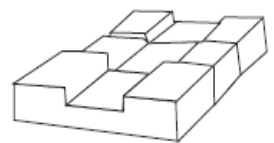

Pit

Figure 2.1: Six Landform classes offered by Peucker and Douglas (1974)

However Dikau's (1989) scheme (Figure 2.2) includes all possible combinations of form thus, is very appropriate for an exhaustive, general geomorphometric classification. It is adopted by many recent authors (Schmidt and Hewitt, 2004; Drăguț and Blaschke, 2006) either fully or partially. Scheme of Dikau (1989) is actually a modification and synthesis of former research by Richter (1962) and Ruhe (1975). Those previous work only described sloping elements of a surface and have no categories for channels, ridges, peaks, etc.

According to this scheme form facets are described with their homogeneous 'slope' and 'aspect', and form elements are defined with their 'slope' and 'curvature'. Above scheme (Figure 2.2) belongs to 'form element' model which describes elementary forms with all possible combinations of slope and curvature. Accordingly slope has two states; flat or sloping. The scheme is divided into two as sloping elements (left) and non-sloping elements (right) in Figure 2.2. Further discrimination is made by curvature. Sloping elements have curvature on plan and profile where curvature has three states; concave, straight and convex, nine sloping form elements were described according to their state of curvature. 

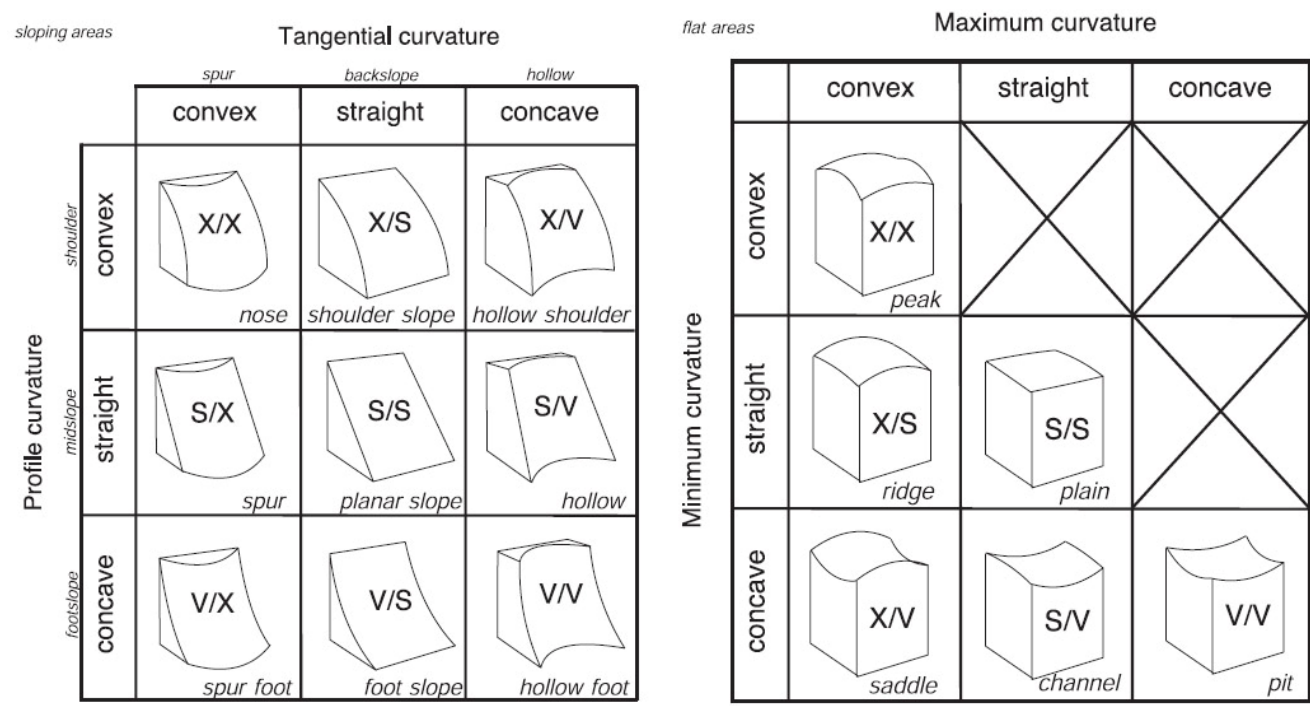

Figure 2.2: Fifteen 'form elements' offered by Dikau (1989) based on local geometry. ( $\mathrm{X}=$ convex, $\mathrm{V}=$ concave, $\mathrm{S}=$ straight $)$.

Dikau (1989) in his classification system defined 'form facets' and 'form elements'.

Non-sloping elements on the other side have slope values near zero where plan and profile curvature values become undefined (Evans, 1972), and minimum and maximum curvature instead are used to describe those elements (Shary, 1995). Features described in the scheme of Dikau (1989) can be thought of as morphometric rather than geomorphometric in that they are characteristic to any surface (Wood, 1996). A more expanded scheme that define a complete system of landform classification according to tangential, profile, mean, difference and total Gaussian curvatures that include the previous classifications of Gauss (1828) and Troeh (1965) has been presented by Shary et al. (2005) (Figure2.3).

This scheme is more sophisticated with more than two curvature indices; however it represents sloping elements only. Moreover this scheme might remain theoretical rather than being applicable as it is not practically very effective to extract these components from DTMs and label them using a generic nomenclature in landform classification context. An important issue not to be neglected is the describing parameters for these DTMs for classification. Given a set of useful DTMs, one should relate them with landform classes. Then a threshold is needed to rule that slope of an image element is satisfactory to be classified as a sloping element or the extent to which an element is convex enough to be classified in to a class e.g peak. Unfortunately there's no explicit and satisfactory criteria defined for general geomorphometric purposes. 


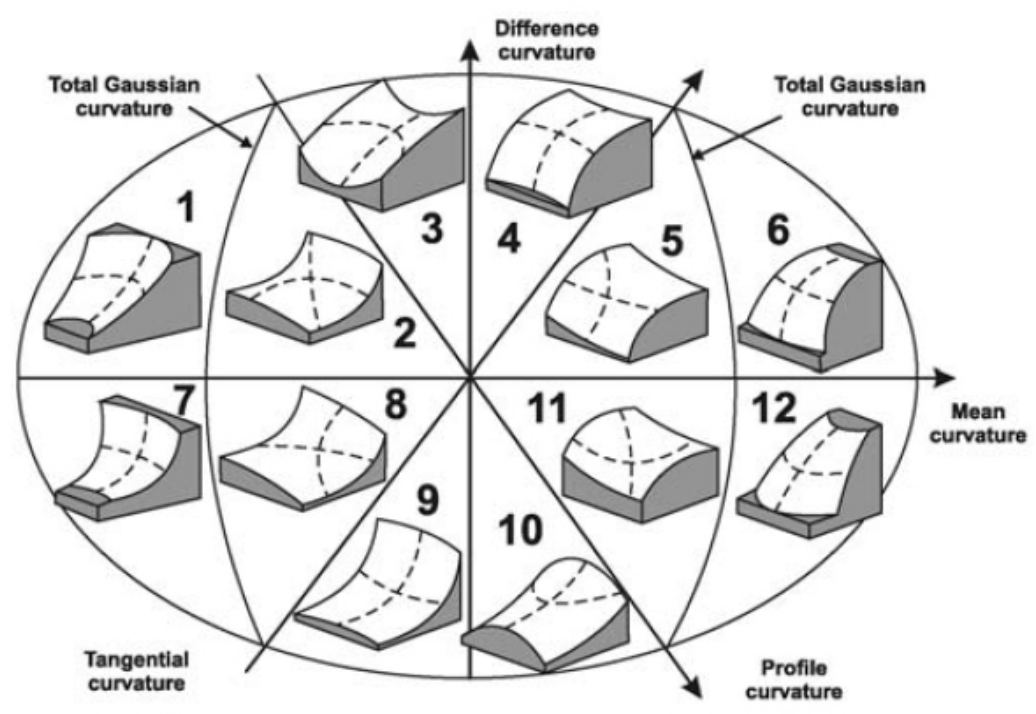

Figure 2.3: Shary's system of landform classification according to signs of tangential, profile, mean, difference and total Gaussian curvatures (Shary et al., 2005).

There are vast amount of slope thresholds to describe flat areas in various studies; e.g: $<5^{0}$ according to Giles, (1998), $<4^{0}$ according to MacMillan and Shary (2004), $<3 \%$ according to Speight (1990),$<3^{0}$ according to Reuter et al. (2006). Curvature criteria are usually very loosely described. Dikau (1989) in his classification scheme for form elements provides a curvature threshold where values lower than of $600 \mathrm{~m}$ radius which is described as $0.16 / 100 \mathrm{~m}$ represents uniformity and values higher represent curvedness. Reuter et al. (2006) suggest $>0.11 / 100 \mathrm{~m}$ for convexity and $<0.11 / 100 \mathrm{~m}$ for concavity for an agricultural landform classification research. Coops et al. (1998) who improved Speight's (1990) classification scheme defined $>0.5$ and $<-0.8 / 100 \mathrm{~m}$ plan curvature for crests and depressions respectively.

\subsubsection{Geomorphometric context}

Local geometry constitutes the basis of a general type of landform classification and it reveals morphometric classes. However, landforms are not random or chaotic, but rather structured as a result of the history of geologic and geomorphic processes which have been acting on the earth surface and produced the current landform (Evans and Cox, 1999; Dehn et al., 2001). Forms have relative positions across the landscape and a structure of organisation, e.g. class 'foot slope' in addition to being a form element with a significant slope and concavity in profile curvature; it is placed at relatively lower positions of the 
landscape. Therefore, those locally delimited landform elements need linkages to wider topographic context (Deng, 2007).

Broadly, two attributes of terrain can be thought of as exhibiting its particular characteristics other than geometry; they are 'relative terrain position' and 'terrain network'.

Relative terrain position approaches threat terrain as a continuous surface and quantitatively describe the relative position of any point, for instance, whether a specific point is it located on the higher or lower positions across the landscape. Terrain is usually classified into categories like upper, mid and lower. Terrain network, depict discontinuities and it subdivides terrain where there is any break in slope or calculates global/local minima or maxima, to detect ridges, basin divides or valley bottoms that constitute the skeleton of the terrain (Dong et al., 2008).

\subsection{Terrain Position}

Shape and topographic position influence flow of surface water, transport of sediments or pollutants and determines climate on local and regional scales. Further, natural phenomena and habitats of plants are directly related to landform patterns and the relative position within a landscape (Blaszczynski 1997; Coops et al. 1998; Blaschke and Strobl 2003). These models are adequate when gravity-induced processes are considered, like e.g. runoff.

Terrain positions are usually labelled as; ridge tops, shoulders, back slopes, foot slopes, etc. Terrain positions depict geographic meanings and spatial processes and they reflect the terrain context.

Locations with the same morphometric properties might belong to different slope positions and are associated with different geomorphic processes, for example, a location on a ridge and a location in a valley might have the same slope gradient or curvature, but their geographic (spatial) context and operating geomorphic processes are different (Qin et al., 2009). Unlike slope and curvature which define morphometry, slope positions convey contextual information which is essential for modelling geomorphic processes and other natural phenomena.

In this section numerous approaches to classify terrain based on relative position are introduced. Most of those schemes were originated from soil-landscape modelling studies. 
Soil-forming processes over the period of soil formation lead to a distinctive, repeated series of soil taxonomic units in the landscape and that idea constructs the basis of the soil catena concept introduced by Milne (1935). Accordingly, at any point in a given landscape, the type and intensity of these processes are dominantly controlled by the redistribution of water, solutes, and sediments by hydrological and microclimatic processes which are in turn, most strongly related to differences in landform shape and position (Pennock and Corre, 2001).

Four-unit slope model of Wood (1942) (Figure 2.4 a) and Nine-unit slope model of (Dalrymple et al., 1968) (Figure 2.4 a) that delimit slope profiles represents typical examples of classifying landscape into relative positions.
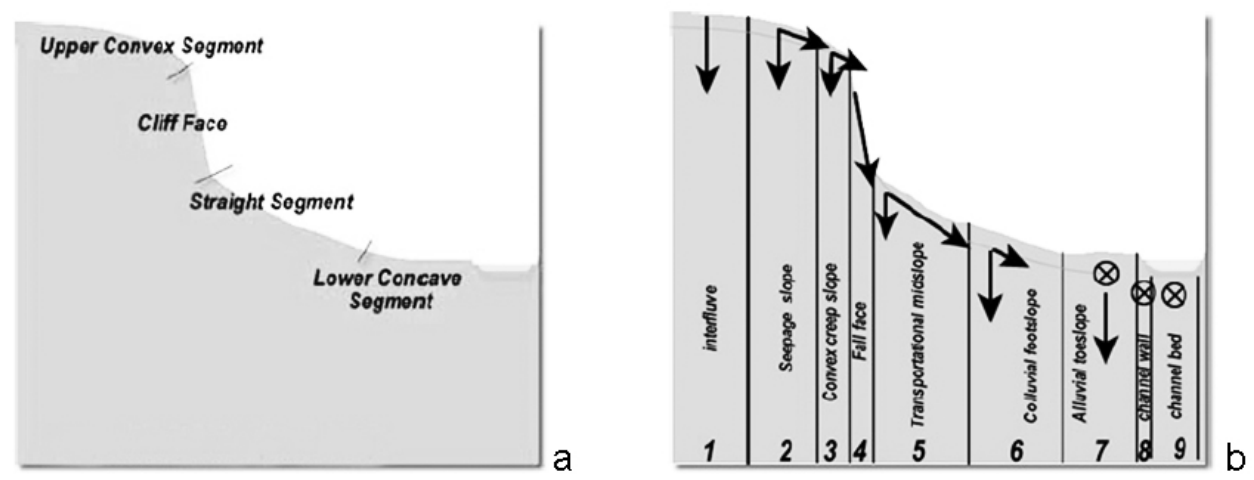

Figure 2.4: a) Slope classification of Wood (1942) b) Slope model by Dalrymple et al., (1968)

Dalrymple et al.'s (1968) model describes each of the nine units by position, slope, profile curvature and actual processes. A list of unit descriptions can be found in Table 2.1 from the study of Conacher and Dalrymple (1977). Units closely relate to the position of a given cell across the landscape. Unlike morphological mapping, units are delimited also by specification of process transitions at the boundaries (Minar and Evans, 2008).

Ruhe and Walker (1968) similarly identified geomorphic units in downslope sequence. They divide hillslope into five segments; summit, shoulder, backslope, foot slope, toe slope, alluvium using slope, slope length and slope width (Figure 2.5).

Those approaches were basically divide a slope profile regarding rate of change in slope on a vertical plane that is represented by cross-sections. Therefore these methods could be 
hardly applied on continuous surfaces and the most important shortcoming was that they simply neglected rate of change in slope on horizontal plane (plan curvature) that described forms like spur and hollow. Ruhe and Walker (1968) also identified geomorphic units of headslope, noseslope and sideslope that add divergent, convergent and linear possibility of plan curvature to their scheme (Figure 2.6).

Table 2.1: Landform units defined by Conacher and Dalrymple (1977).

\begin{tabular}{|l|l|l|}
\hline \multicolumn{2}{|l|}{ Land-surface unit } & Characteristics \\
\hline 1 & Interfluve & $\begin{array}{l}\text { Interfluve with predominant pedomorphologic processes caused by } \\
\text { vertical (up and down) soil-water movements; } 0-1^{\circ} \text { slope gradient }\end{array}$ \\
\hline 2 & Seepage slope & $\begin{array}{l}\text { Upland area where responses to mechanical and chemical eluviation } \\
\text { by lateral subsurface soil-water movements predominate }\end{array}$ \\
\hline 3 & $\begin{array}{l}\text { Convex creep } \\
\text { slope }\end{array}$ & $\begin{array}{l}\text { Convex slope element where soil creep is the predominant process, } \\
\text { producing lateral movement of soil materials }\end{array}$ \\
\hline 4 & Fall face & $\begin{array}{l}\text { Areas with gradients greater than } 45^{\circ} \text { characterized by the process of } \\
\text { fall and rockslide }\end{array}$ \\
\hline 5 & $\begin{array}{l}\text { Transportational } \\
\text { midslope }\end{array}$ & $\begin{array}{l}\text { Inclined surfaces with 1-45 }{ }^{\circ} \text { gradients and responses to transport of } \\
\text { large amounts of material downslope by flow, slump, slide, erosion } \\
\text { and cultivation }\end{array}$ \\
\hline 6 & $\begin{array}{l}\text { Colluvial } \\
\text { footslope }\end{array}$ & Concave areas with responses to colluvial redeposition from upslope \\
\hline 7 & Alluvial toeslope & $\begin{array}{l}\text { Areas with responses to redeposition from upvalley alluvial materials; } \\
0-4^{\circ} \text { gradient }\end{array}$ \\
\hline 8 & Channel wall & A channel wall distinguished by lateral corrosion by stream action \\
\hline 9 & Channel bed & $\begin{array}{l}\text { A stream channel bed with transportation of material downvalley by } \\
\text { stream action as the predominant process }\end{array}$ \\
\hline
\end{tabular}

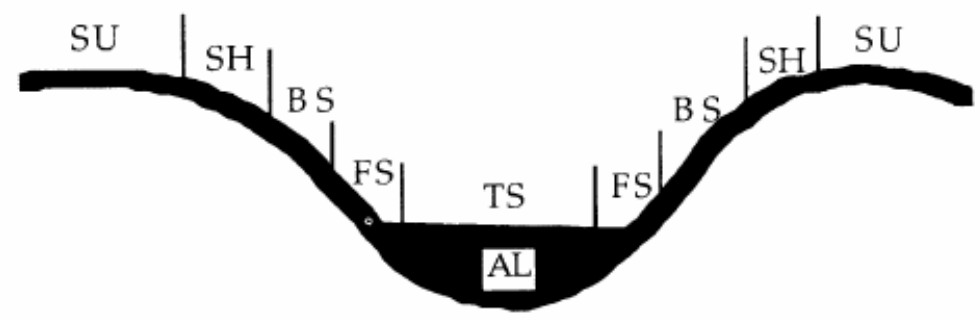

Figure 2.5: Geomorphic units offered by Ruhe and Walker (1968). (SU: summit, SH: shoulder, BS: backslope, FS: foot slope, TS: toe slope, AL: alluvium) 


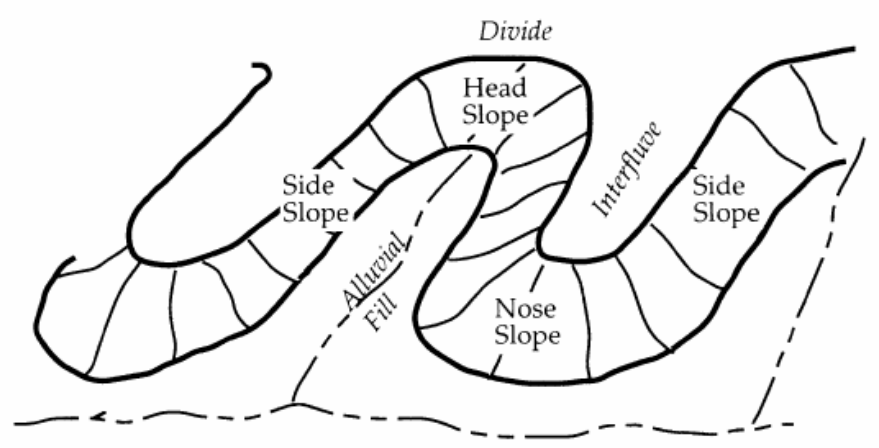

Figure 2.6: Geomorphic units offered by Ruhe and Walker (1968) (in Ventura and Irvin (2000)).

Similarly, Hugget (1975) presented a soil landscape system, which overcame the twodimensional character of the catena principle and simulated the behavior of a three dimensional system (Figure 2.7)

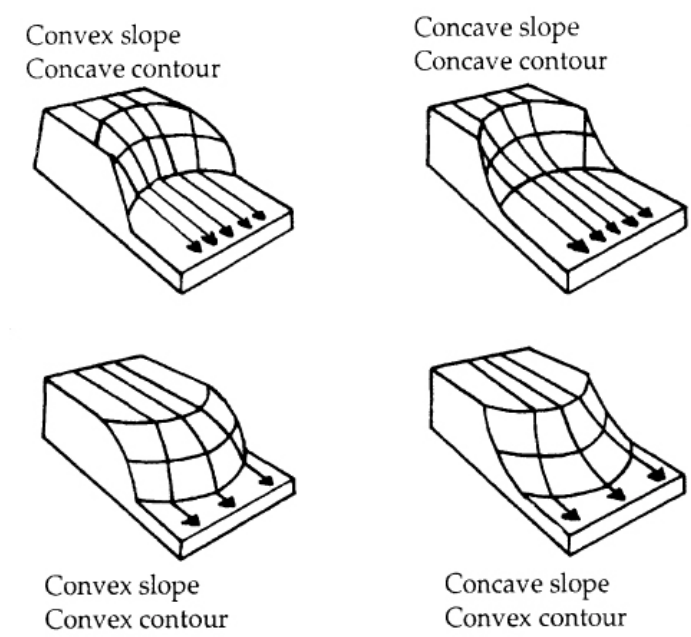

Figure 2.7: Basic slope shapes with surface flow lines (Huggett 1975), source: Ventura and Irvin (2000).

Pennock et al. (1987) used data published by Young (1972) to classify nine threedimensional landform elements by measures of slope, plan and profile curvature (Figure 2.8). Elements were described with rate of change of slope in both vertical and horizontal plane. This scheme is also compatible with the scheme of Richter (1962) and Ruhe (1975) and Dikau (1989); Figure 2.2 (left side). 


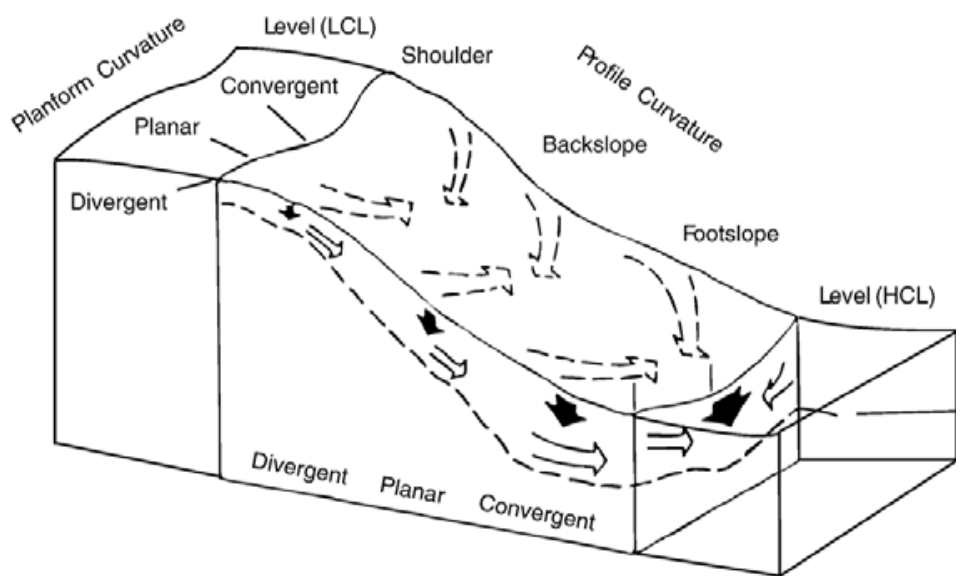

Figure 2.8: Geomorphic units offered by Pennock et al. (1987).

An extension of Pennock's (1987) definitions is presented by MacMillan and Pettapiece (2000). The level class was replaced by six separate units to differentiate level areas and depressions in upper, mid and lower landscape positions respectively. An extra unit was added in both the mid-slope and lower-slope landscape positions that were neither convex nor concave, but rather planar in the across-slope direction. The final 15 classes are listed in Table 2.2 .

Table 2.2: Fifteen landform units proposed by MacMillan and Pettapiece (2000).

\begin{tabular}{|lll|}
\hline $\begin{array}{l}\text { Landform } \\
\text { category }\end{array}$ & Landform element & Description \\
\hline Upper slope & Level crest & Level area in Upper slope \\
& $\begin{array}{l}\text { Divergent shoulder } \\
\text { Upper depression }\end{array}$ & $\begin{array}{l}\text { Convex upper, watershedding element } \\
\text { Depression in upper slope position }\end{array}$ \\
\hline Midslope & Backslope & Rectilinear transition mid-slope segment \\
& Divergent backslope & Sloping "ridge" \\
& Convergent backslope & Sloping "through" \\
& Terrace & Level mid-slope $>2 \mathrm{~m}$ above base level \\
& Saddle & Special case of a divergent footslope \\
& Midslope depression & Depression in midslope position \\
\hline Lower slope & Footslope & Concave, water receiving element \\
& Toeslope & Rectilinear in lower slope $>20 \%$ of low slope \\
& Fan & Special case of a divergent toeslope \\
& Lower slope mound & Crown in lower slope $<2$ m above base level \\
Level lower slope & Level in lower slope, $>20 \%$ of low slope \\
Depression & Concave element in lowest landform position \\
\hline
\end{tabular}


A key Australian classification of landforms was developed by Speight $(1974,1990)$ who proposed a two-level descriptive procedure for a systematic and parametric description of landforms into landform patterns and landform element. Table 2.3 provides classification system of profiles across the terrain divided into morphological types of landform elements as classified by Speight (1990).

Most of the approaches mentioned, especially the earlier schemes that subdivide slope in downslope sequence were adopted from manual measurement of cross-sections. Even if they had descriptions on three dimensions they did not address particular techniques to extract them out of digitally represented terrain i.e. DEMs and DTMs.

Table 2.3: Topographic position classes by Speight (1990).

\begin{tabular}{|l|l|}
\hline Name & Topographic position classes \\
\hline Crest & $\begin{array}{l}\text { Area high in the landscape, having positive plan and/or profile } \\
\text { curvature }\end{array}$ \\
\hline Depression (open, closed) & $\begin{array}{l}\text { Area low in the landscape, having negative plan and/or profile } \\
\text { curvature, closed: local elevation minimum; open: extends at } \\
\text { same or lower elevation }\end{array}$ \\
\hline Slope & Areas having a slope $<3 \%$ \\
\hline Simple slope & $\begin{array}{l}\text { Planar element with an average slope }>1 \% \text {, subclassified by } \\
\text { relative position }\end{array}$ \\
\hline Upper slope & $\begin{array}{l}\text { Adjacent below a crest or flat and adjacent above a flat or } \\
\text { depression }\end{array}$ \\
\hline Mid slope & $\begin{array}{l}\text { Adjacent below a crest or flat but not adjacent above a flat or } \\
\text { depression }\end{array}$ \\
\hline Lower slope & $\begin{array}{l}\text { Not adjacent below a crest or flat and not adjacent above a flat or } \\
\text { depression }\end{array}$ \\
\hline Hillock & $\begin{array}{l}\text { Not adjacent below a crest or flat but adjacent above a flat or } \\
\text { depression }\end{array}$ \\
\hline Ridge & $\begin{array}{l}\text { Compound element where short slope elements meet at a narrow } \\
\text { crest }<40 \mathrm{~m}\end{array}$ \\
\hline
\end{tabular}

Classification approaches that delineate landscape into relative position categories recently being explored are index-based terrain attributes. They combine the environmental meanings of terrain shape and position (Schmidt and Dikau, 1999; Wilson and Gallant, 2000) and have been attractive in representing particular characteristics of topography (Deng et al., 2007). Some of the indices that are used to categorize terrain into positions generated out of DEMs are; 'topographic position index' of Jenness (2006), 'local elevation' 
of Hatfield (1999), 'relative hillslope position' of (Behrens et al., 2009). Those methods will be mentioned in Section 2.3.2 (DTMs).

Some authors use breaks of slope to categorize slope units (Giles, 1998), however it is not likely that one can grab a continuous line that connects breaks of slope using slope data or other DTMs derived out of DEMs.

Index-based techniques were adopted by some recent authors to organize landforms, i.e. Schmidt and Hewitt (2004) differentiated spurs and ridges which are very similar in form, using a valley/hill index, Dragut and Blaschke (2006) used relative altitude to obtain upland midland and low land classes.

\subsection{Terrain Network}

Discontinuities of terrain surface i.e.; specific features like break-lines or extrema points across the landscape has long been recognised to contain important topological information that characterises a surface. In general terms, terrain network can be defined as set of statements that identify the connections between points on a surface and the nature of the edges that connect those (Wood, 2000). Terms such as 'weighted surface network' (Pfaltz, 1976; Wolf, 1988) also called 'Pfaltz-graph' by following researches, 'metric surface network (Rana and Wood, 2000), 'surface specific features' (Fowler and Little, 1979), 'ridge and channel network' (Werner, 1988), Drainage and Divide Network (Bennett, Armstrong, 1996), 'surface topology' (Wood and Rana, 2000), 'terrain skeleton' (Clarke, 1990; Dong et al., 2008), are used almost analogously and will be referred to as 'terrain networks' throughout.

Terrain network is extracted based mainly on discontinuities. Surface is traced where there is any break in slope or global/local minima or maxima is calculated to detect ridges, basin divides or valley bottoms that constitute the terrain network (Dong et al., 2008).

There are broadly three approaches each originated from different concepts but deal with surface network features. They are; topologic approach, hydrographic approach and morphometric approach.

\section{Topologic Approach}

Terrain network is represented in a topological structure. Local minima and maxima points are extracted, and they are connected to each other to construct a network regarding a set of 
topological rules. Those models are known to be used for generalization purposes (Mackaness and Beard, 1993; Wolf, 1989) and they are widely used out of geomorphometry, i.e., in modelling industrial surfaces. In a Terrain Network; vertices/points represent pits, passes, and peaks, the edges/lines represent channels and ridges and the edges can have weights specifying the differences in elevations if it is a weighted surface network. A representative Weighted Surface Network by Wolf (1991) where he modelled connectivity relationships using graph theory is given in Figure 2.9.

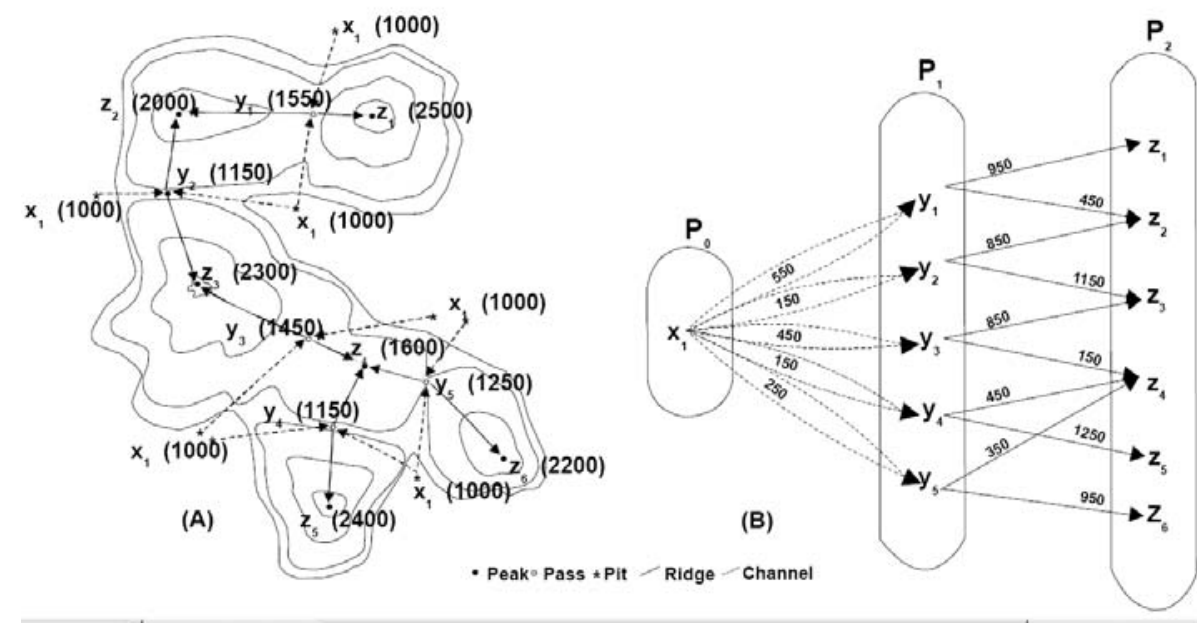

Figure 2.9: (a) Ridges and channel lines of a topographic surface (number in parentheses indicate altitude of the point) (B) Tripartite graph representing the topological structure of the surface; edge weights are indicated on the edges (Wolf, 1991).

A topologic network of terrain surface has several local minima and maxima and other similar surface-specific elements, also known as morphologically important points (Rana, 2004). The most important surface-specific elements as described by Cayley are (1859)

- pits: local minima (pits or bottoms of depressions, holes);

- peaks: local maxima (tops of hills and mountains, summits);

- ridge lines: lines connecting points that are local maxima in transverse section;

- course lines: lines connecting points that are local minima in transverse section (channels, flow lines);

- passes: crossing points of ridge and course lines;

- break lines: where the slope change is sudden

Abstract models of topographic surfaces should satisfy the some properties suggested by Pfaltz 1976) and Wolf(1998) such as: 
- intersection of ridges and channels is not allowed.

- channels connect pits and passes, and ridges connect peaks and passes.

- number of pits minus the number of pass points, plus the number of peaks must always be two.

- from all passes exactly two channels and exactly two ridges originate

An illustration on a topographic map represents an implementation of those rules on a terrain topology that is fitted to the topography (Figure 2.10).

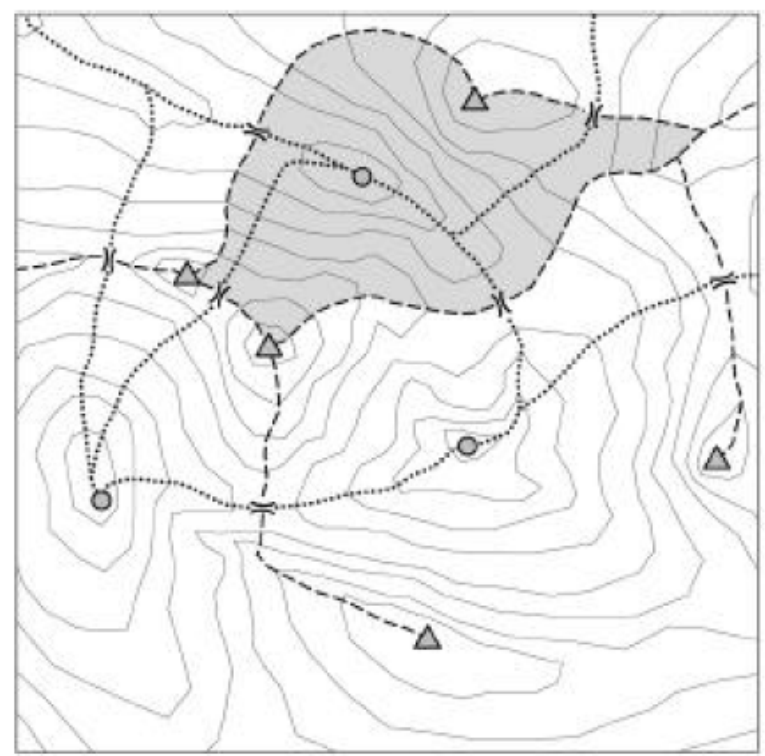

Xass

$\circ$ Pit

$\triangle$ Peak

W. Valley Line

八. Ridge Line

Contour

Figure 2.10: Terrain topology on a contour map by Schneider (2003). A drainage area is highlighted.

\section{Hygrographic Approach}

Hydrographic approach models the surface flow across the landscape. A flow direction and flow accumulation is produced where each hypothetic rain drop flows to a direction and accumulates into another cell. Where flow accumulation per pixel exceeds a minimum threshold is described as flow path that construct a stream network or drainage pattern across the landscape. Flow paths are usually put into hierarchal orders of magnitude (Horton, 1945; Strahler, 1964; Shreve, 1966). 


\section{Morpholometric Approach}

Terrain network constitutes surface specific features of the terrain, i.e. peak, ridge, saddle, channel, pit. Those elements are also morphometric features (Dikau, 1989) and can be extracted using morphometric DTMs that describe local geometry. However features gathered this way hardly have topological consistency and are not connected e.g. there will be gaps along channels and ridge lines. Outcome of morphometric approach is also scale dependent. Schemes that describe morphometric forms have been mentioned in previous section (2.2.3.1, Morphometry). Technical details and algorithms about these methods will be mentioned in Section 2.3 (DTMs).

\subsubsection{Scale}

Scale in the wide range of use refers to multiple concepts, thus it is an ambiguous term. Scale may refer to

(i) Cartographic scale: Depicted size of a feature on a map relative to its actual size in the world

(ii) Generalization: Level of abstraction;

(iii) extent of the area

(iv) Phenomenon scale: size at which human or physical earth structures or processes exist, regardless of how they are studied or represented;

(v) Analysis scale: Size of the unit at which some problem is analyzed, particularly when geographers work with digital representations of the earth's surface i.e. DEMs and DTMs in GIS environment (Montello, 2001; Hengl and Evans, 2009)

However, there are also strong interrelations between concepts that these terms refer to. For instance; in cartography, maps are produced at different scales, e.g; 1:100,000, that is; a ground area of $1 \times 1 \mathrm{~km}$ will be a mapped as $1 \times 1 \mathrm{~cm}$ at a scale of 1:100,000. A map at $1 / 100,000$ scale and 1/10,000 scale has different levels of abstraction, they can be used as source for different analysis scales and they can be interlinked to different phenomena scale.

If we consider the earth we live in, scale would range from a single sand grain to the whole earth crust. There is vast amount of terms that describe ranges of scales; for instance use of terminological continua such as 'local-global' or 'micro-meso-macro' scale is commonly used by geographers (Montello, 2001). 
Determination of scale of a study is very crucial. One should evaluate what is the appropriate scale for the analysis. According to Wood (2009), this is clearly depends to an extent on the nature of the application and inherent scale of features under study by the researcher. This may be already determined as a phenomena scale where a specific physical or biophysical event takes place or the researcher may search for characteristic scale of the study of interest.

Even not strictly determined with upper and lower limits, natural phenomena usually operate functionally at particular scale range or ranges. Those scales can be approximately represented using a set of data produced at the relevant scale. On the other hand, size and spacing of landforms often show clustering around characteristic scales (Evans, 2003). One can search for those scales and perform the analysis with or without necessarily relating it with particular phenomena. Analysis scale can be directly adjusted according to the phenomena scale or a characteristic scale is examined. In this context, scale will be mentioned in terms of phenomena scale and characteristic scale.

Scale is the window of perception hence, it has a direct influence on the landform type on a particular location. As for instance, at a finer scale topography can be perceived as a small peak, however when the window is enlarged it becomes a part of planar slope and at a coarser scale even a channel as in Figure 2.11.

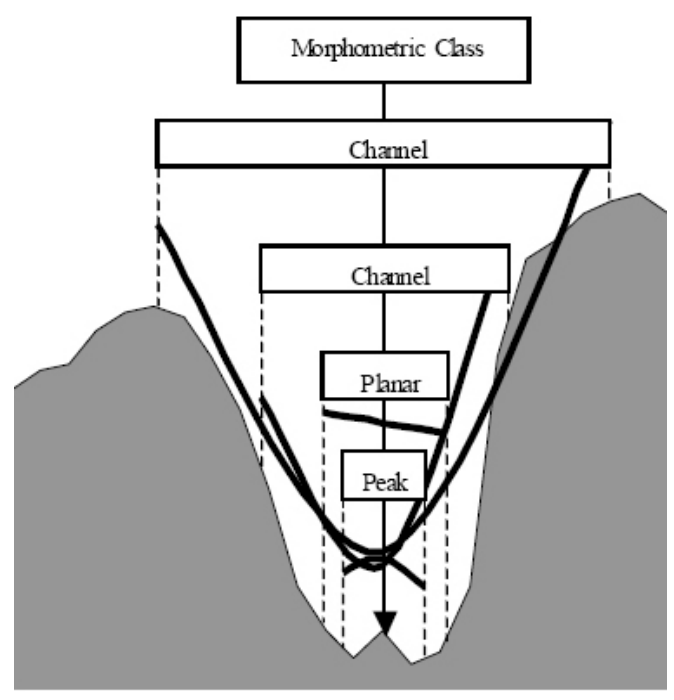

Figure 2.11: Changing morphometric classes with scale (Fisher et al., 2005) 
If a morphometric class is observed to be persistent in the same area of geographical space at different spatial resolutions than it is recognised as scale-independent. Changes to a landform's classification with resolution provides information on the characteristics of that landform, and how its function in the landscape changes when viewed at different resolutions, describing the allometry of a class (Bull, 1975). A morphometric class can be viewed as isometric if its shape and form do not change with scale (Arella et al., 2007).

\subsubsection{Phenomena scale}

There are many physical processes on the earth surface and they operate at different orders of magnitudes. Wide review on the literature suggests that some scale ranges are very commonly referred to as 'Landscape scale', 'hillslope scale' microrelief' and 'mesorelief'. They are described as the scales where any particular phenomena or a set of different physiologic and biologic phenomena are evidently observed. These scales can not be said to fall into exact same range of scales, however they are within a range in between local and global, or micro and macro relief. Landscape scale is a scale range commonly employed in soil-landscape modelling, ecology and environment management disciplines. Hillslope scale is common in hydrology and soil science, microrelief and mesorelief is common in geomorphology as briefly described below;

Landscape scale is described as the scale range where ecological units are defined by general topography, geomorphic process, surficial geology, soil associations, and potential natural communities, patterns, and local climates (Forman and Godron 1986). Landscape comprises of many nested or overlapping ecological processes that can be observed at a space at a time (Bailey, 1996).

At the landscape scale relief is an important feature because, the surface energy budget (evapotranspiration and photosynthesis rate) correlates highly with topography through solar radiation and temperature (e.g. Moore et al. 1993b), climatic conditions change with the altitude, topographic attributes depict a high degree for correlation with soil attributes (Gessler et al. 1995; Gessler \& Ryan 1999; Moore et al. 1993a), as a conclusion, many important ecological processes operate primarily at landscape scales (Dunning et al., 1992). According to the definition in ECOMAP (1993), a landscape is 'a heterogeneous land area composed of a cluster of interacting ecosystems that is repeated in a similar form throughout; and can be viewed at one time from one place'. ECOMAP is a derivative of Bailey's (1996) ecoregional approach that focuses on terrestrial aspects of land 
environments including land form and some other landform-controlled parameters like climate and current vegetation.

Land Types (LT) or Ecologic Land Types (ETLs) and Land Type Associations (LTAs) are commonly used to refer to fundamental conceptual units for ecological modeling at the landscape scale. LTs are subdivisions of LTAs based on similarities in soils, landform, geomorphic processes, and plant associations (ECOMAP, 1993). LTs are most relevant to ecological analysis, understanding and manipulation at landscape scale. Therefore they are of crucial importance to ecologists and natural-resources managers. Topographic factors constitute a major part in delineating LTs.

As designed by the National Hierarchical Famework of Ecological Units (NHFEU) (Clealand et al., 1997), general size of LTs range from ten to hundreds acres which correspond to approximately $10^{5}$ to $10^{6} \mathrm{~m}^{2}$, just pertaining to the Mesorelief as described by Dikau (1989) and MacMillan (2004). LTs are are described by: landform and topography (elevation, aspect, slope gradient, and position) Phases of soil subgroups, families, or series Rock type, geomorphic process , plant associations (Clealand et al., 1997)

Hillslope scale is widely pronounced in hydrological studies. Hydrological responses show systematic down slope variations due to the water and soil material and nutrient movement controlled by surface forms along the hillslope (Park and Giesen, 2004). In general, local scale, hillslope scale and where most of the are often used to distinguish different spatial scales in hydrology (Schmidt et al., 2000). The smallest scale at which landscape evolution can be studied reasonably is the hillslope scale (Martin and Church, 2004). All hillslopes have a general morphological similarity by definition and they are bounded by a lower slope and a crest at the top. Hillslopes together constitute the drainage basin surface. This distinctive form indicates a distinctive process unit. Sediment is transported from the upper portions of the hillslope and is deposited along the slope base by run off (Martin, 2000).

Hillslope is a finer scale than landscape scale according to (Phillips, 2005) and it is equivalent to 'Microrelief' (Dikau, 1989) according to Schmidt and Hewitt (2004).

Following terms relate to relief classification that describes scale ranges introduced by Dikau (1989) associated with different geomorphologic processes. 
Micro-meso relief scale: Landform features range from small landforms, such as cirques, to large or coarse-scale landforms, such as mountain ranges (Rasemann et al., 2004). Levels of relief units at ranges of scales described by Dikau (1989) are very informative for understanding scales and their relevant landforms provided with particular measures of quantification.

According to Dikau (1992); whole land surface is subdivided into six exhaustive scale ranges namely; picorelief, nanorelief, microrelief, mesorelief, macrorelief, megarelief, from finest to coarsest respectively. Following figure illustrates a description of the complete system where relief forms / geomorphography are given in order of size, taxonomical hierarchy and time dimension with some representative examples (Figure 2.12). Landforms according to hierarchical levels of relief units by Dikau (1989) and corresponding examples of landforms are shown in Table 2.4 and a more detailed version including microrelief and mesorelief with representative attributes is given in Table 2.5. Dikau's (1989) definitions of scale ranges of relief associated with landforms (Table 2.5) were illustrated for Switzerland by Rasemann et al., (2004) (Figure 2.13).

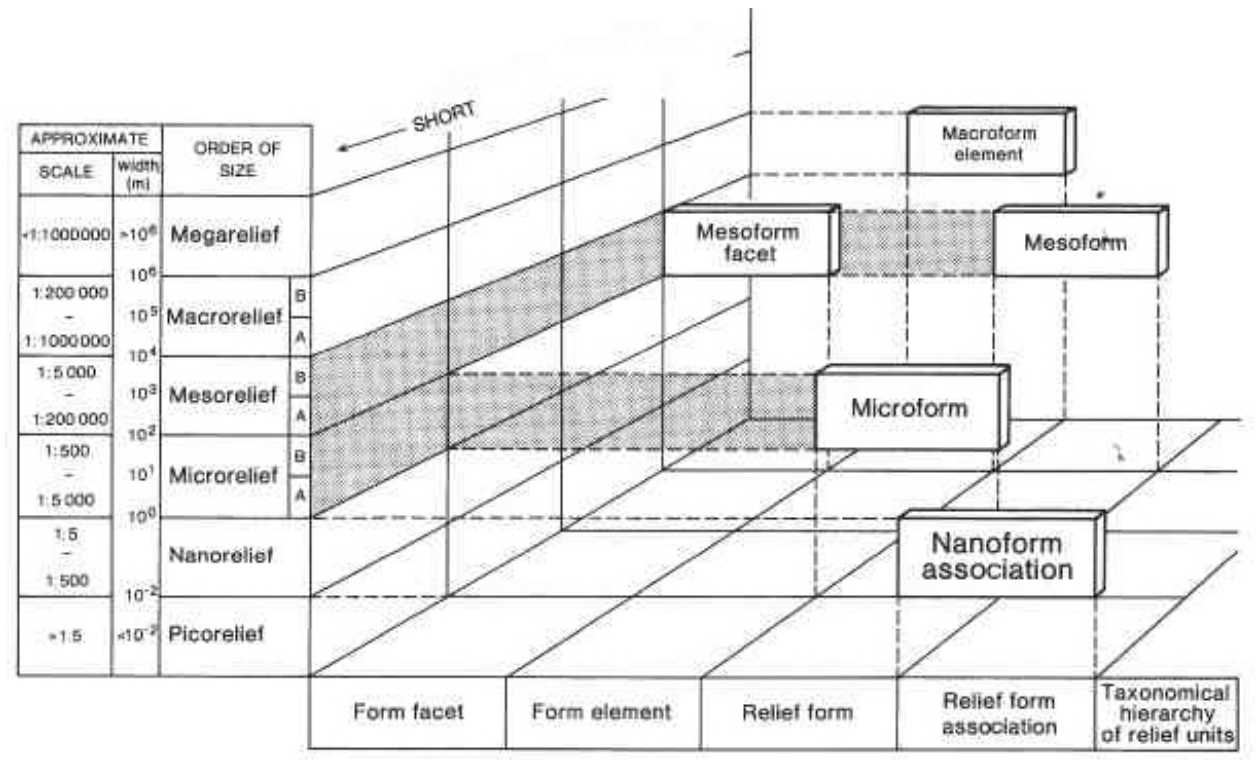

Figure 2.12: Hierarchy of geomorphographic units of differing complexity derived from hierarchy theory (Dikau, 1992). 
Table 2.4: Dimensions of relief units at hierarchical levels and corresponding examples of landforms (Dikau, 1989).

\begin{tabular}{|c|c|c|c|c|c|}
\hline \multirow[b]{3}{*}{ Megarelief } & & \multicolumn{3}{|c|}{ Dimension of landform } & \multirow[b]{2}{*}{ Example landforms } \\
\hline & & $W(\mathrm{~m})$ & $A\left(\mathrm{~m}^{2}\right)$ & $H / D(\mathrm{~m})$ & \\
\hline & & $>10^{6}$ & $>10^{12}$ & & Canadian shield \\
\hline Macrorelief & $\begin{array}{l}\text { B } \\
\text { A }\end{array}$ & $-10^{5}$ & $10^{10}$ & $>10^{3}$ & $\begin{array}{l}\text { Mountain area, the Alps, the } \\
\text { Rhinegraben }\end{array}$ \\
\hline Mesorelief & $\begin{array}{l}\text { B } \\
\text { A }\end{array}$ & $-10^{3}$ & $10^{6}$ & $-10^{2}$ & $\begin{array}{l}\text { Valley, moraine hills, cuesta } \\
\text { scarps }\end{array}$ \\
\hline Microrelief & $\begin{array}{l}\text { B } \\
\mathrm{A}\end{array}$ & $-10^{1}$ & $10^{2}$ & $10^{\circ}$ & $\begin{array}{l}\text { Gully, ice wedge, doline, } \\
\text { dune, terrace }\end{array}$ \\
\hline Nanorelief & & & & & Karren, tafoni, erosion rills \\
\hline Picorelief & & $<10^{-2}$ & $<10^{-4}$ & & Glacial striations \\
\hline
\end{tabular}

Table 2.5: Attributes of hierarchical levels of relief units: Microrelief and Mesorelief (Dikau, 1992).

\begin{tabular}{|c|c|c|}
\hline \multirow{2}{*}{$\begin{array}{l}\text { Hierarchic level of } \\
\text { relief unit/ } \\
\text { characteristic dimension }\end{array}$} & \multicolumn{2}{|c|}{ A selection of primary attributes } \\
\hline & $\begin{array}{l}\text { Object attributes } \\
<<\text { inherent }>>\end{array}$ & $\begin{array}{c}\text { Toposequences/patterns } \\
<<\text { context }>>\end{array}$ \\
\hline $\begin{array}{l}\text { Mesorelief B: } \\
\text { Scale: 1:50,000 - 1:200,000 } \\
\text { DEM: } 100-600 \text { m (approx.) }\end{array}$ & $\begin{array}{l}\text { Attrlbute distribution, } \\
\text { Attrlbute variabillty, Grain, Relief, } \\
\text { Type of toposequences, } \\
\text { Ridgeichannel density }\end{array}$ & $\begin{array}{l}\text { Type of low level unit, } \\
\text { Position within macrorellef, } \\
\text { Type of inhomogeneity, } \\
\text { Process pattern }\end{array}$ \\
\hline $\begin{array}{l}\text { Mesorelief A: } \\
\text { Scale: 1:5,000 - 1:50,000 } \\
\text { DEM: } 20-300 \mathrm{~m} \text { (approx.) }\end{array}$ & $\begin{array}{c}\text { Grain, Texture, } \\
\text { Shape, } \\
\text { Gradlent, } \\
\text { Curvature, Aspect }\end{array}$ & $\begin{array}{c}\text { Toposequence position, } \\
\text { Pattern of low-level units, } \\
\text { Neighbours, } \\
\text { Functlonal relationshlps }\end{array}$ \\
\hline $\begin{array}{l}\text { Microrellef A/B: } \\
\text { Scale: 1:500-1:5000 } \\
\text { DEM: } 1 \text { - } 50 \text { m (approx.) }\end{array}$ & $\begin{array}{c}\text { Size, } \\
\text { Shape, } \\
\text { Gradient, Aspect, } \\
\text { Curvature }\end{array}$ & $\begin{array}{c}\text { Connectlveness, } \\
\text { Density, Dispersion, } \\
\text { Position within mesoreflef }\end{array}$ \\
\hline
\end{tabular}




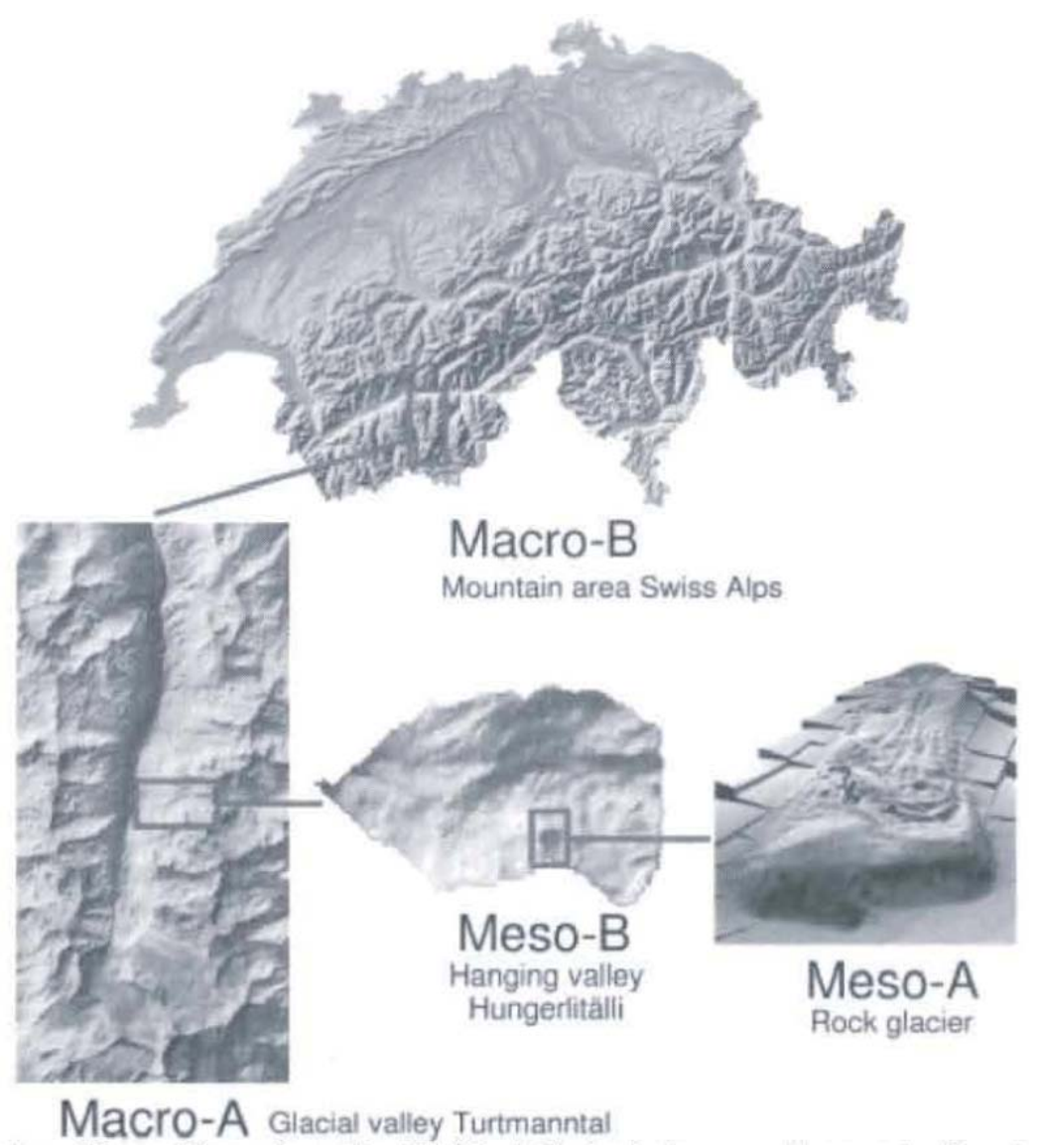

Figure 2.13: Landform scales depicting nestedhierarchy in Switzerland (Rasemann et al., 2004).

However, every discipline perception may have its own purpose and perception window, socalled phenomenon scale, and scale will differ depending on the problem formulation and the specific hypotheses being tested (Hurlbert, 1984). The assumption is that the measured value for the phenomenon can be reliably associated with the landform element (Pennock and Corre, 2001).

Anderson and Burt (1990 in Schmidt, 2000) for instance relates geomorphic features with hydrologic processes (Figure 2.14). Authors note that; dominant features of each discipline in a spatial and spatio-temporal context, translating scale properties from one discipline to the other is an open research question.

Figure 2.14 shows dominant features of each discipline in a spatial and spatio-temporal context, translating scale properties from one discipline to the other is an open research question (Anderson and Burt, 1990 in Schmidt, 2000.) 


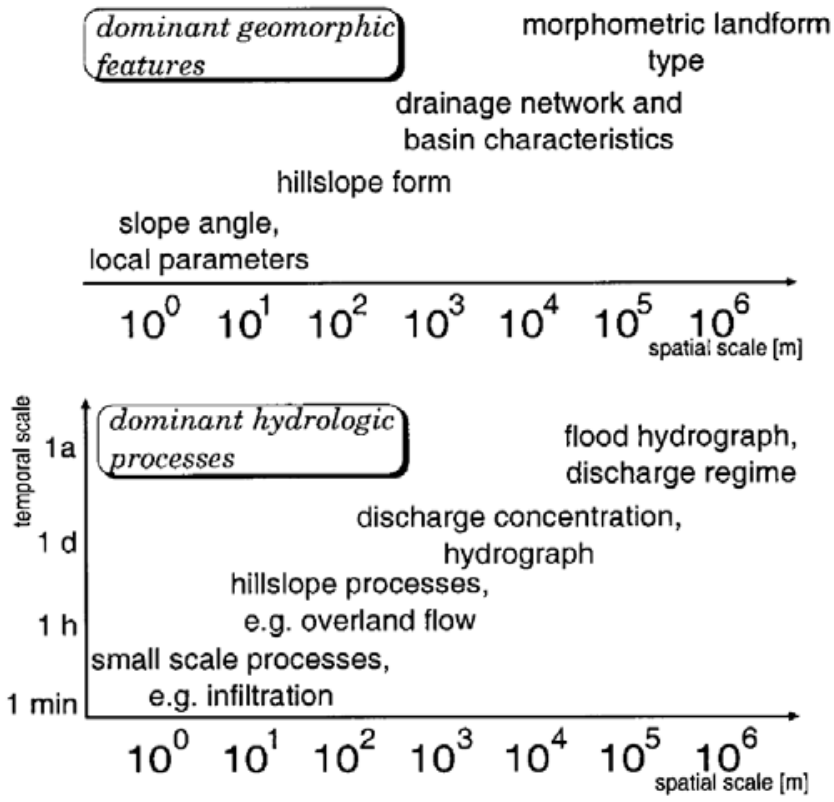

Figure 2.14: Corresponding scales in hydrology and geomorphology (Anderson and Burt, 1990 in Schmidt, 2000)

As a result it can be said that mesorelief seems to cover a scale range that is common to most of the disciplines and its described by set of input parameters like for instance 1/5000 to $1 / 5000$ is appropriate source for studying mesorelief with approximately 20 to $300 \mathrm{~m}$ DEM resolution. That is also in the range to map at landscape/LT scale (MacMillan et al., 2004) (Figure 2.15).

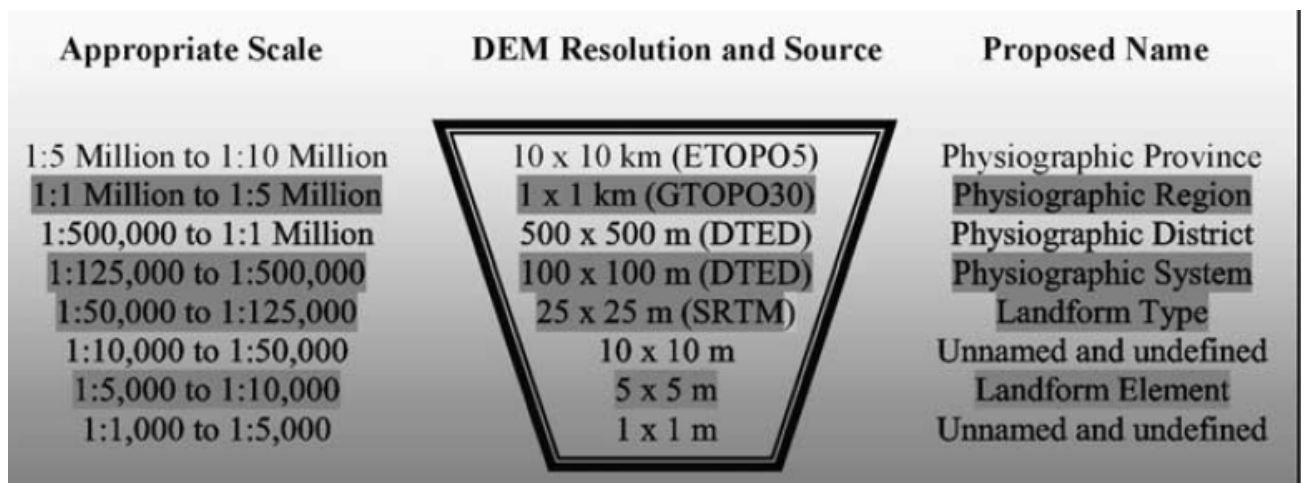

Figure 2.15: Hierarchy of landform entities (MacMillan et.al, 2004). 


\subsubsection{Characteristic Scale}

In any classification attempt to derive landforms one should consider multi-scalar nature of landscape. A peak on a local scale may be negligible if one could look at it on a regional scale because, interpretation and identification of landforms is inherently multi-scalar (Strobl, 2007). Any location can be allocated to a specific class, but the class to which a location is assigned may change with the window of perception, hence scale of the observation (Figure 2.16) giving rise to ambiguity and so vagueness. (Skidmore 1990; Fisher et al., 2004). The question is 'is the statement that this location is a peak correct under all observations?' The answer is presumably no.
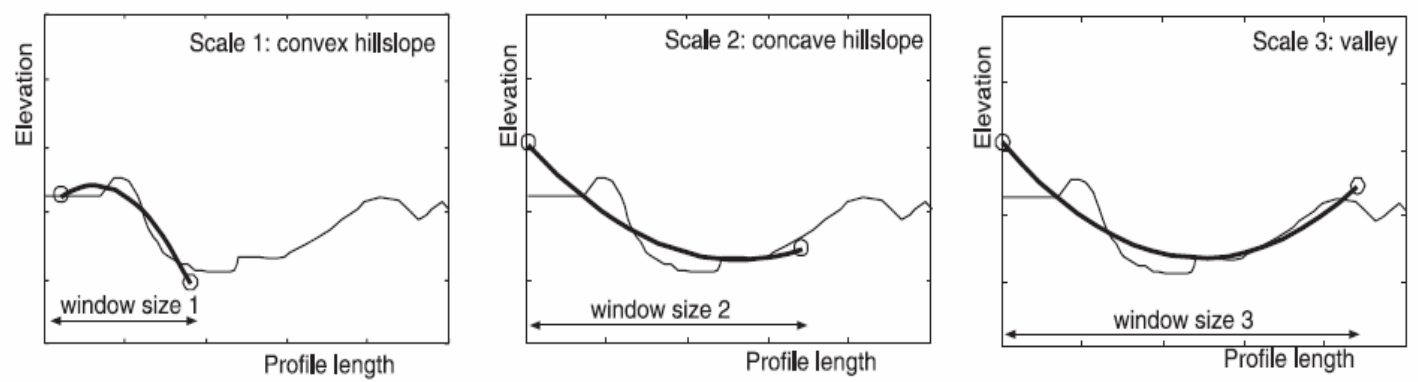

Figure 2.16: Effect of window size enlargement on a specific location on a hilly terrain.

Most of the studies relate scale with a specific phenomenon, e.g. Schmidt and Hewitt, (2004) who evaluated correlation of profile curvature calculated at various window sizes with soil depth measurements and employed the window size that yields best correlation.

Geomorphic phenomena that shape the terrain is also scale-specific, hence size and spacing of landforms are clustered around characteristic scales or limits related to either processes that operate on them or space available (Evans, 2003; Wood, 2008). Evans (2003) demonstrated that most of the geomorphologic forms depict specificity to particular scale ranges and nature of any one scale signature can be used to identify characteristic scales at which a surface parameter is strongest. According to Wood (2009), terrain parameters may pose $\mathrm{s}$ higher values at specific ranges, if these scales described with window sizes are plotted against level-specific indicators, scale signatures are obtained. These signatures may exhibit some appropriate scales for analysis. 
In the past, DEMs were considered to represent scale however, window of perception or window size to caldulate DTMs are more proper way of exhibiting scale as the DEM resolution available is already fine, and it is possible to produce scale levels through multiscale analysis, either in a cell- or an object-based environment. Expanding window size is widely employed to represent scale (e.g. Mackay et al. 1992; MacMillan et al. 2000, Schmidt and Hewitt 2004)

\subsection{Digital Representation of Terrain}

The basis for terrain analysis and landform classification has conventionally been topographic maps, airphotos and field observations (e.g. Cayley, 1859; Maxwell, 1870). Those methods heavily depend on expert knowledge and visual interpretation skills combined with sort of manual or semi automated techniques. To utilize quantitative analysis and classification of terrain, digital representations of terrain which were capable of numerically representing the terrain surface were developed. Digital Elevation Model (DEM) constitutes the basis of all quantitative representations of a terrain surface. The term Digital Terrain Model (DTM) often implies landscape attributes rather than the elevation of the surface, where the term DEM is preferred for models that contain elevation data only (Burrough and McDonnell 1998).

\subsubsection{DEM}

A DEM consists of elevation values assigned to location coordinates of the land surface and forms the basic dataset for any further digital terrain modelling. Elevation values in a DEM are either in regular or irregular form, where regular DEM is also called raster-based or grid-based and irregular DEM is vector-based so called TIN (Weibel and Heller, 1991; Rana, 2004). Triangular Irregular Network (TIN) is widely known model for irregularly spaced elevation points, whereas DEM is typically referred to as a regular gridded matrix (Figure 2.17).

Most widely accepted description of Digital Elevation Model is made by Burrough (1986) accordingly; DEM is described as "digital representation of the continuous variation of relief over space". Throughout this study DEM is referred to as regularly gridded matrix of elevation values of a land surface. 


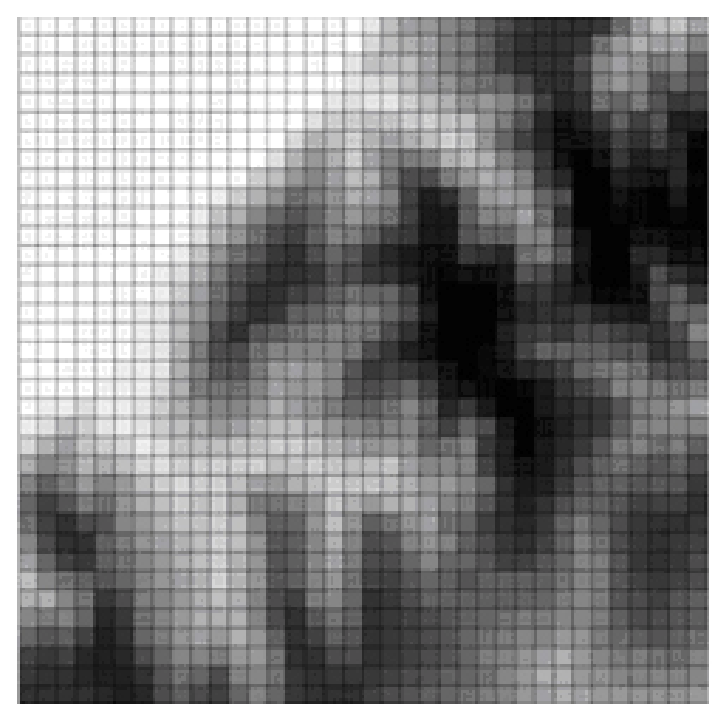

Figure 2.17: A typical DEM.

There are various sources of information to construct a DEM. They can be broadly categorized into three, according to Nelson et al. (2009).

(i) Ground survey techniques: The accurate surveying of point locations ( $x, y, z$ values). There are both traditional and high-tech ground survey techniques

(ii) Existing topographic maps: Deriving contours from hardcopy topographic maps that are originated Photogrammetric stereo interpretation

(iii) Remote sensing: The interpretation of image data acquired from airborne or satellite platforms. There are conventional methods to: (1) Photogrammetric/ stereo methods (including both airborne and satellite); and (2) Laser (mostly airborne at present, but will be from satellites in the future); and (3) Radar (both airborne and satellite using interferometry).

Different sources of DEM data and different data structures associated with those source information need further attention to construct DEM in a proper way since different data structures require different methods and algorithms of production and refinement of DEMs.

\section{DEM Source Data Types}

DEMs can be generated out of different types of topographic data. These can be broadly categorized into three groups as suggested by Hutchinson and Gallant (2000); (i) surface- 
specific point elevation data, (ii) contour and stream line data, (iii) remotely sensed elevation data. These data have different structures and different DEM generation techniques are applicable for each.

\section{Surface-specific point elevations data}

Surface-specific point elevation data refers to the points that are critical on the topography like peak, pit, saddle, points on streams and points on ridges which form the skeleton of the terrain (Clarke 1990). Therefore surface specific points can be used to characterize (Werner 1994) and generalize terrain surface (Wolf 1988; Rana and Wood 2000). These data can be obtained by ground survey and by manually assisted photogrammetric stereo models. Ground survey data however is more common ever since GPS enhanced the accuracy and availability of ground surveyed data, yet still not easy to cover large extents of areas (Hutchinson and Gallant, 2000). Another way of obtaining Surface-specific point elevation data is to extract it from DEMs, however this requires a DEM in the first place. Surfacespecific point elevation data gathered this way is typically used in topological surface representations (Pfaltz, 1976; Wolf, 1988) and to assist building Triangular Irregular Network (TIN).

\section{Contour data}

Contour data together with some important elevation points (e.g. on hill tops) and streamlines constitute the basis of topographical maps. Contour data digitized from topographical maps are still the most widely used terrain data source for large extents of areas (Hutchinson and Gallant, 2000; Li et al., 2004). A contoured topographic map is perhaps the most common way of representing terrain. A topographic map usually contains important features that are orthogonally projected onto a 2D horizontal plane (Li et al., 2004), where surface altitude is represented with contours/isolines passing through each every specific interval generated automatically from photogrammetric stereo models.

It is believed that the contour map is one of the most important inventions in the history of mapping due to its convenience and being easily perceived (Li et al., 2005). They are very suitable for displaying purposes, however they are not particularly suitable for numerical analysis or modelling (Burrough, 1996). The reason is, terrain as a continuous phenomenon can not be adequately represented with discrete features like contour lines. In a contour map, there are typically gaps in between isolines/contours without any information. This shortcoming has been the motivation for development of techniques to estimate elevation 
values at no-data locations using the measurements taken at surveyed or mapped features. These techniques are known as "interpolation techniques".

There are a great variety of methods available for terrain surface interpolation (e.g. Inverse Distance, Kriging, Minimum Curvature, Polynomial Regression, Triangulation, Nearest Neighbour, Moving Average, Local Polynomial, etc.). Watson (1992) has broadly identified two types of interpolation methods for terrain surfaces: (i) fitted functions and (ii) weighted averaging.

'Local polynomial' and 'minimum curvature' are commonly used fitted functions. Minimum curvature is a Spline-based method where a minimum-curvature surface is fitted through the input features. The interpolation produces a continuous and smooth surface; however rapid changes in slope may occur in vicinity of the data points or contour lines.

Inverse Distance Weighted (IDW) and Kriging algorithms are common weighted averaging interpolations. Inverse distance weighted is a linear interpolation technique. Elevation values of unsampled points are estimated with the surrounding sampled points, that is inverse distance weighted average of the point elevations within a search window. Kriging is also a linear interpolation technique, where the estimated value is a weighted average of the surrounding sampled values. The value of variable $Z$ at an unmeasured location is estimated using a minimized estimation variance derived from a semi-variogram model.

The quality of DEMs can be improved by using an appropriate interpolator. However there is no universal technique that is clearly superior to others, and appropriate for all sampling techniques and DEM applications (Weibel and Heller, 1991; Li et al., 2005). Mitas and Mitášová (1999) evaluated various interpolation approaches to produce DEM, and concluded that the most important aspects are how well smoothness and tension are described, and how well streams and ridges are incorporated.

For instance IDW is a very common method in estimating any measurement across the space e.g. rate or pollutants or amount of nutrients measured at specific points across the land of interest. Altitude across the landscape varies continuously however it has particular characteristics, it is not random or chaotic but is has a particular system, it has structural elements like streams and ridges. Therefore interpolation of terrain measurements requires further interest. 
A procedure based on the thin-plate spline technique devised by Hutchinson (1989) creates hydrologically correct DEM using a multi-resolution iterative finite difference interpolation method. This method is called ANUDEM (Australian National University Digital Elevation Model), and later adopted into the Topogrid program available in ESRI products (ArcGIS Desktop Help 9.3). The Topogrid program is a process of interpolating grid DEMs from contour line data or scattered point elevation data. This method has a drainage enforcement algorithm that automatically removes spurious sinks and imposes constraints that ensure (i) a connected drainage structure, and (ii) correct representations of ridges and streams from input contour data.

\section{Remotely sensed Elevation Data}

DEMs can be generated by stereoscopic interpretation of aerial photographs, satellite images or directly from Air/spaceborne scanners.

\section{Stereo aerial photography and satellite images}

Most of the currently available digital elevation datasets are the product of photogrammetric data capture (Moore et al. 1991). Manual or automatic stereo plotters are used for stereoscopic interpretation of aerial photographs and satellite images to generate DEMs (Wilson and Gallant, 2000). In particular stereo images from SPOT, JERS-1 and ASTER satellites have been used for extracting topographic information (e.g. Hirano et al. 2003) using similar techniques. However, use of high resolution visible and infrared satellite sensors is limited due to atmospheric conditions, cloud cover and temporal resolution and they produce coarser DEMs compared to other sources.

\section{Air-and spaceborne scannerS}

It is possible to gather altitude data directly by remote sensing. Radar Interferometry and Laser-scan are the two new technologies that measure altitude of surface directly and have the potential to reduce current shortcomings in accuracy (Maune, 2001) and they can provide broad spatial coverage.

\section{Radar interferometry}

An emerging technology which utilizes Synthetic Aperture Radar (SAR) sensors that are capable of obtaining land surface information independent of weather conditions and external illumination source (e.g. Terunuma et al. 2005; Canisius et al., 2009). SAR interferometry or INSAR technique measures the amplitude and phase of the signal from SAR data using a pair of SAR images from the same area, acquired with slightly different 
sensor positions. Radar interferometry (InSAR or IfSAR) yields terrain-height accuracies and resolutions comparable with those generated by optical methods (Hanssen, 2001). Shuttle Radar Topographic Mission (SRTM) that uses the interferometric technique to estimate terrain altitude reached a recent coverage $80 \%$ of all the land on Earth (Shuttle Radar Topography Mission, 2010). DEMs from SAR are becoming more available with Radarsat, Envisat, and ALOS.

\section{Laser-Scan}

Other emerging technology; known as Laser scan (LS) or Laser altimetry; in particular LiDAR (light distance and ranging) provide DEMs of fine resolution and very high accuracy (Pike, 2002). LiDAR, a relatively new technology, uses the emission and reflection of light pulses usually from an airborne scanner. One of the most attractive characteristics of LiDAR is its very high vertical accuracy i.e. $1 \mathrm{~m}$ resolution and the other is laser beams can penetrate the vegetation cover (Clark et al., 2004).

The data collected by active systems may not only require interpolation, but also considerable processing to obtain the resultant DEM. Because these systems measure the elevations with systematic and random errors, these errors can be both due to limitations of the observing instruments and/or surface slope and roughness (Dixon, 1995; Wilson and Gallant, 2000). Processing has a big influence on the accuracy of the DEM as well. Processing methods also require precise Ground Control Points (GCPs) to minimize the systematic error, that are not always very easy to locate especially in remote and mountainous regions.

\subsubsection{Digital Terrain Models (DTMs)}

Digital Terrain Models (DTMs) quantitatively describe characteristics of form and structure of the land surface (Dong et al., 2008). While term 'DEM' is used for representing the variability of altitude across the landscape, the term DTM often implies landscape attributes that relate to terrain, but not necessarily elevation (Burrough and McDonnell 1998). DTM, Terrain Attribute (TA), terrain parameter, are the terms that are being used interchangeably and they all refer to quantitative measures of terrain. Attributes that quantify terrain properties are referred to as DTMs throughout this study. Examples of DTMs are slope, aspect, and the rate of change of these expressed by profile and plan curvature, the surface 
topology of run-off, compound indices like wetness, ridges, streams, distance to ridges or streams, and other more complex attributes like incident solar radiation (Burrough, 1998).

There is a vast amount of DTMs from different views gathered using different algorithms that represent different characteristics of the land surface. There have been attempts to categorize DTMs according to their calculation methods, spatial extents or purpose of use. Wood (1996) distinguished DTMs as general and hydrological according to application domain, where general DTMs quantify morphometry. DTMs were classified into single and composite DTMs (Wilson et al., 2000), local and non-local (Florinsky, 1998), local, regional, global (Shary et al., 2002), first order, second order (Wilson and Gallant, 2000), local and regional/combined (Behrens et al. 2009).

Regarding the previous attempts of categorization of DTMs and scope of this study, DTMs are categorized broadly as morphometric and geomorphometric where geomorphometric DTMs involve relative position and terrain topology models.

\subsubsection{Morphometry/Local Geometry}

Local geometry of the landscape constitutes the fundamental component of terrain units. Form; not necessarily pertaining to a land surface but any other surface is effectively represented by means of change in the variation of elevation with its first and second order derivatives; slope and curvature respectively. Therefore, among various DTMs; 'slope' and 'curvature' are the two indispensable fundamentals of the study of morphometry.

Local geometry of a surface is calculated based on one of the two major approaches. First approach calculates the terrain parameter at a specific location using finite difference approximation (e.g. Tobler, 1970; Eyton, 1991). Second approach fits a polynomial equation e.g. least square polynomial or a quadratic, to the land surface to approximate local geometry for the specific location (e.g. Young, 1978; Evans, 1980; Zvenbergen and Thorne, 1987). Local quadratic approximation of surface as specified by Evans (1972) and Young (1978) is widely used to derive morphometric DTMs and demonstrated more accurate terrain derivatives compared to other common methods (Florinsky 1998). Quadratic approximations are calculated at specific local window/kernel. In polynomial-fitting based algorithms, kernel is considered similar to a set of regularly spaced points, each one typically derived from the geometric centre of the kernel cells. The result is assigned to a 
cell in the output raster, which is located at the same place as the kernel's central cell (Rana, 2006).

'Slope' and 'curvature' are first and second derivatives of DEM respectively. Slope is the rate of change of elevation in both $\mathrm{x}$ and $\mathrm{y}$ directions and is used to identify the direction and magnitude of steepest gradient (Jones, 1998). Curvature is the measure of convexity or concavity of terrain surface.

The measurement of second derivative of elevation i.e. curvature is more problematic than slope and aspect, since the partial derivative of the bi-variate quadratic yields an infinite number of solutions, surface convexity must therefore be expressed in some defined direction. Evans (1979) separates curvature into two orthogonal components; profile and plan convexity, former being the vertical component in the direction of aspect and latter being the horizontal component. These measures can only be calculated if the slope normal is not vertical. For this special case, two alternative measures are used; minimum and maximum curvature (Evans, 1979).

Terrain curvature for a particular cell represents the curvature of a line formed by intersecting a plane of some chosen orientation with the terrain surface.

profile curvature is the curvature of a line formed by intersecting a plane with the plane of the $\mathrm{Z}$ axis and aspect direction, similarly;

plan curvature; the plane intersects with the xy plane

(Evans, 1972).

For the flat topography the plan and profile curvature becomes null because they remain undefined for quadratic patches with zero gradient (Evans, 1972). For these cases minimum and maximum curvatures are used as a substitute to represent curvature of a line orthogonal to the xy plane which intersects with xy plane (Shary, 1995). The plane for which minimum curvature is calculated is orthogonal to the plane of the maximum curvature and visa versa. To describe curvature of a surface geometrically, the concept of a normal section is useful.

Let ' $n$ ' be a normal vector (at the point $\mathrm{X}$ ) to the surface S (Figure 2.18). 


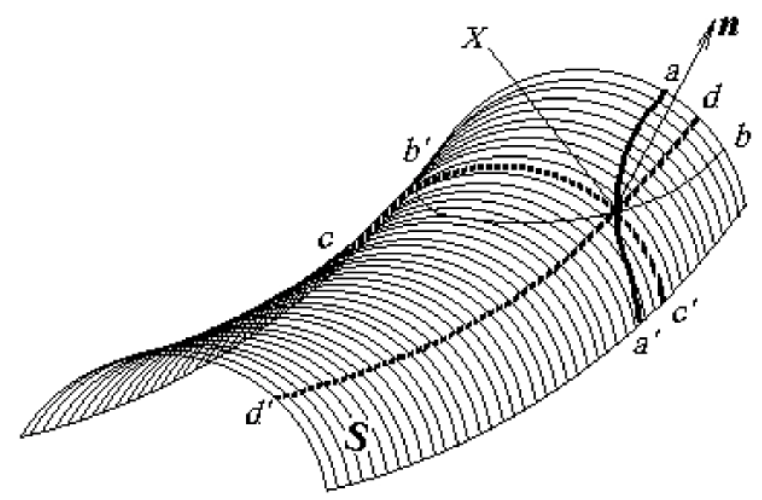

Figure 2.18: Normal sections of four curvatures marked on a hypothetical surface ' $S$ '.

$\mathrm{n}$ : normal vector to $\mathrm{S}$ at the point $\mathrm{X}$. aa': normal section of profile curvature bb': normal section of plan curvature (also hypothetic contour line), cc'and dd' normal section of maximum and minimum curvatures respectively (adapted from Shary et al., 2002).

First; gravitational field marks at point $\mathrm{X}$ two normal sections; tangential to the contour line (bb') is normal section of plan curvature or horizontal curvature which is very similar, and perpendicular to it, along which water flows (aa') is normal section of profile curvature. The form of the surface itself marks two other normal sections on it having a maximum of its curvature (cc') and that having a minimum (dd') among all possible normal sections at the point X (Shary et al. 2002). Maximum and minimum curvatures sections has no enforced directions, they are rather the maximum and minimum curvatures of normal sections at a specific location. Minimum and maximum curvatures are used to describe geometrical forms like ridge, peak and pit. Dikau (1989) in his classification scheme proposes the use of slope and four curvature parameters namely; plan, profile, minimum, maximum curvatures to categorize terrain into landform classes (Figure 2.2).

A moving window is passed over a DEM and change in gradient and a central point in relation to its neighbours is derived by a second-order polynomial function the best fitting quadratic surface passing through that window is estimated using least-squares regression. Slope and curvature are calculated by taking first derivatives and partial second derivatives of a bi-quadratic polynomial representing expression a local patch of a surface (Wood, 2009) (Eq. 2.1):

$$
Z=a x^{2}+b y^{2}+c x y+e y+f
$$

Where $\mathrm{x}, \mathrm{y}$, and $\mathrm{z}$ are local coordinates, and $\mathrm{a}, \mathrm{b}, \mathrm{c}, \mathrm{d}, \mathrm{e}, \mathrm{f}$ are quadratic coefficients. 
To solve this equation (Eq. 1) the number of points sampled; 'i' must be at least 6 . Minimum number would be 9 if sample of elevation values are limited to a square window of $3 \times 3$ centered around the point of interest, 25 if window is $5 \times 5, \ldots \mathrm{n}^{2}$ if window is $\mathrm{nxn}$, where $\mathrm{i}=9$, the resultant quadratic expression will be identical to Evans' quadratic derivation (Evans, 1979). 6 coefficients are expressed as functions of the 9 cells in a $3 \times 3$ local window arranged as follows with elevation values $\mathrm{z} 1$ to $\mathrm{z} 9, \mathrm{z} 5$ being the central cell, with a grid size of $g$ (figure 2.19):

\begin{tabular}{|l|l|l|}
\hline$Z_{1}$ & $Z_{2}$ & $Z_{3}$ \\
\hline$Z_{4}$ & $Z_{5}$ & $Z_{6}$ \\
\hline$Z_{7}$ & $Z_{8}$ & $Z_{9}$ \\
\hline
\end{tabular}

Figure 2.19: Elevation values in a $3 \times 3$ local window from $\mathrm{Z} 1$ to $\mathrm{Z} 9$.

Six coefficients are calculated as follows:

$\mathrm{a}=(\mathrm{z} 1+\mathrm{z} 3+\mathrm{z} 4+\mathrm{z} 6+\mathrm{z} 7+\mathrm{z} 9) / 6 \mathrm{~g} 2-(\mathrm{z} 2+\mathrm{z} 5+\mathrm{z} 8) / 3 \mathrm{~g} 2$

$\mathrm{b}=(\mathrm{z} 1+\mathrm{z} 2+\mathrm{z} 3+\mathrm{z} 7+\mathrm{z} 8+\mathrm{z} 9) / 6 \mathrm{~g} 2-(\mathrm{z} 4+\mathrm{z} 5+\mathrm{z} 6) / 3 \mathrm{~g} 2$

$\mathrm{c}=(\mathrm{z} 3+\mathrm{z} 7-\mathrm{z} 1-\mathrm{z} 9) / 4 \mathrm{~g} 2$

$\mathrm{d}=(\mathrm{z} 3+\mathrm{z} 6+\mathrm{z} 9-\mathrm{z} 1-\mathrm{z} 4-\mathrm{z} 7) / 6 \mathrm{~g}$

$\mathrm{e}=(\mathrm{z} 1+\mathrm{z} 2+\mathrm{z} 3-\mathrm{z} 7-\mathrm{z} 8-\mathrm{z} 9) / 6 \mathrm{~g}$

$f=(2(z 2+z 4+z 6+z 8)-(z 1+z 3+z 7+z 9)+5 z 5) / 9$

(Evans, 1979)

Then first and second derivatives DEM are calculated as shown in equations from Eq 2.2 to Eq.2.7.

$$
\begin{gathered}
\text { slope }=\arctan \left(\sqrt{d^{2}+e^{2}}\right) \\
\text { aspect } \arctan \frac{e}{d} \\
\text { plan curvature }=-\frac{\left(b d^{2}+a e^{2}-2 c d e\right)}{\left(e^{2}+d^{2}\right)^{3 / 2}} \\
\text { profile curvature }=-\frac{\left(a d^{2}+b e^{2}+2 c d e\right)}{\left(e^{2}+d^{2}\right)\left(1+d^{2}+e^{2}\right)^{3 / 2}}
\end{gathered}
$$




$$
\begin{aligned}
& \text { Maximum curvature }=\left(-a-b+\sqrt{\left((a-b)^{2}+c^{2}\right)}\right) \\
& \text { Minimum curvature }=\left(-a-b-\sqrt{\left((a-b)^{2}+c^{2}\right)}\right)
\end{aligned}
$$

This simplification is the least squares solution for 3 by 3 case, however, a matrix solution is required for the more general case of an nxn local window (Wood, 1996) (Eq 2.8).

$$
Z_{i}=b_{0}+b_{1} x_{i}+b_{2} y_{i}+b_{3} x_{i}^{2}+b_{4} x_{i} y_{i}+b_{5} y_{i}^{2}
$$

The six parameters are calculated by multiplying the $9 \times 1$ vector of altitudes by a $6 \times 9$ matrix of coefficients: matrix inversion is unnecessary (Evans, 1979). To solve the general case, the unknown coefficients are expressed as a set of 6 normal equations (Unwin, 1975) (Eq2.9):

$$
\begin{aligned}
& b_{0} N+b_{1} \sum x_{i}+b_{2} \sum y_{i}+\quad b_{3} \sum x_{i}^{2}+b_{4} \sum x_{i} y_{i}+\quad b_{5} \sum y_{i}^{2} \quad=\sum_{z_{i}} \\
& b_{0} \sum x_{i}+\quad b_{1} \sum x_{i}^{2}+\quad b_{2} \sum x_{i} y_{i}+\quad b_{3} \sum x_{i}^{3}+\quad b_{4} \sum x_{i}^{2} y_{i}+b_{5} \sum x_{i} y_{i}^{2} \quad=\sum_{z_{i}} \cdot x_{i} \\
& b_{0} \Sigma y_{i}+b_{1} \Sigma x_{i} y_{i}+\quad b_{2} \Sigma y_{i}^{2}+b_{3} \Sigma x_{i}^{2} y_{i}+b_{4} \sum x_{i} y_{i}^{2}+\quad b_{5} \Sigma y_{i}^{3}=\sum_{z_{i}} \cdot y_{i} \\
& b_{0} \sum x_{i}^{2}+\quad b_{1} \sum x_{i}^{3}+b_{2} \sum x_{i}^{2} y_{i}+\quad b_{3} \sum x_{i}^{4}+b_{4} \sum x_{i}^{3} y_{i}+b_{5} \sum x_{i}^{2} y_{i}^{2} \quad=\sum z_{i} \cdot x_{i}^{2} \\
& b_{0} \sum x_{i} y_{i}+b_{1} \sum x_{i}^{2} y_{i}+b_{2} \sum x_{i} y_{i}^{2}+b_{3} \sum x_{i}^{3} y_{i}+b_{4} \sum x_{i}^{2} y_{i}^{2}+b_{5} \sum x_{i} y_{i}^{3}=\sum z_{i} \cdot x_{i} y_{i} \\
& b_{0} \Sigma y_{i}^{2}+b_{1} \sum x_{i} y_{i}^{2}+\quad b_{2} \Sigma y_{i}^{3}+b_{3} \sum x_{i}^{2} y_{i}^{2}+\quad b_{4} \sum x_{i} y_{i}^{3}+\quad b_{5} \sum y_{i}^{4} \quad=\sum z_{i} \cdot y_{i}^{2}
\end{aligned}
$$

'Landserf' (Wood, 1996) a freeware GIS provides a set of tools to derive morphometric and other DTMs based on quadratic approximation. Wood (1996) customized Evans's (1972) algorithm to approximate surface form for window sizes larger than $3 \times 3$ and provided a set of tools to derive fine or coarse scale DTMs in Landserf.

\subsubsection{Geomorphometric context}

\subsection{Terrain Position}

Almost all of the approaches to describe the position of a particular location across the landscape subdivide terrain into slope positions as mentioned in detail in section 2.2.3.2.1 (Relative Terrain Position). However those methods rather provide an insight about terrain position, and position has been loosely defined to characterize the local topography in relation to its spatial context. Terrain position is usually described with terms such as 
hillcrest, upper slope, foot slope, depression etc. However these terms are commonly used without any quantitative criteria to define them. Moreover those methods simply lack adaptation to surfaces represented by DTMs, as they date back to a period before DTMs, an are commonly represented on cross sections. But after the advent of DEM and DTMs, there have been many efforts to quantify and classify terrain position with particular DTMs. This section is allocated to present those approaches. Speight's work $(1974 ; 1990)$ for instance was one of the most recognized and widespread as it has been materialized for the soillandscape modelling in whole Australia. However, criteria for subdivision into slope classes (upper, mid and lower slope) as defined by Speight (1990) are not provided. One reason may be that the models have not been sufficiently defined with quantitative terrain properties (Klingseisen , 2004). Delineation of terrain positions e.g. crest, slope, and depression was made on topographic maps and field survey for decades. However, need to develop algorithms that can generate terrain positions according to the models used in field survey has emerged with the availability of DTMs and an adaptation of methodological approaches into application via transfer of rules came forward.

There are many approaches that utilize DTMs to describe terrain position. These approaches utilize DTMs of quite different nature and motivation of use. According to the DTMs that are used, approaches can be categorized as based on 'local elevation', 'surface flow' and 'proximity to terrain network' and there are also approaches that combine DTMs of different types which can be referred to as combined approaches.

\section{Local elevation}

Local variation in topography can be useful to consider the nature of each point with reference to a wider context (Blaszczynski, 1997). Terrain position quantification based on local elevation is basically comparison of elevation point with the elations in a local window of a given size. Some of the representative techniques are described below.

Percentile: Ranking of the point at the centre of the window relative to all points in the window gives a measure of relative highness or lowness of a point of interest across the others within a window (Gallant and Wilson, 2000). It is simply calculated by counting the number of points lower than the central point, the percentile ranges in between $0-100,0$ indicating the lowest position and 100 the highest. However it doesn't quantify the magnitude of highness or lowness. 
Difference from Mean Elevation: The difference between elevation at the centre of the local window and the mean elevation in the window is a measure of the relative terrain position of the point of interest (Gallant and Wilson, 2000).

Diff $=Z_{0}-\bar{Z}$

$Z_{0}=$ elevation of the model point under evaluation

$\bar{Z}=$ mean elevation within the window

Topographic Position Index (TPI) is an adaptation of this method which compares the elevation of each cell in a DEM to the mean elevation of a specified neighborhood around that cell. Local mean elevation is subtracted from the elevation value at centre of the local window. Algorithm is provided as an ESRI script by Jenness Enterprises (Jenness, 2006), and it has local window options of; rectangular, circular and annulus.

$\mathrm{TPI}_{\mathrm{i}}=Z_{0}-\frac{\sum_{1-n} Z_{n}}{n}$

Where;

$\mathrm{Z}_{0}=$ elevation of the model point under evaluation

$Z_{n}=$ elevation of grid within the local window

$\mathrm{n}=$ the total number of surrounding points employed in the evaluation

Positive TPI values represent locations that are higher than the average of the local window e.g. ridges. Negative TPI values represent locations that are lower e.g. valleys. TPI values near zero are either flat areas (where the slope is near zero) or areas of constant slope (where the slope of the point is significantly greater than zero), high positive values relate to peaks and ridges.

\section{Index of Landscape Position}

A similar method developed by Fels (1995) for ecological purposes yields a quantitative index of landscape position (LPOS) by evaluating elevation differences between a given point and other distance-weighted model points within a specified search radius. Specifically, landscape position is calculated as:

$\operatorname{LPOS}=\frac{\sum_{1-n} \frac{\left(Z_{n}-Z_{0}\right)}{d}}{n}$

Where;

$\mathrm{Z}_{0}=$ elevation of the model point under evaluation 
$Z_{n}=$ elevation of grid within the local window

$\mathrm{d}=$ horizontal distance between the two model points

$\mathrm{n}=$ the total number of surrounding points employed in the evaluation

The value calculated is the mean of the distance-weighted elevation differences between a given point and all other model points within a specified search radius. Greater positive values indicate lower topographic positions (proximal to streams) and greater negative values indicate higher landscape positions (ridges, summits) while values approaching zero indicate mid-slope positions. Where relief is minimal within the search radius, values will also tend to approach zero.

Those methods besides being robust and simple, they are sensitive to size of the local window. Using a small window produces fine outputs, which is not favourable; for instance suggests use of local windows at least at hill slope size. Fels and Matson (1996) suggest that radius of search distance should be one-half of the fractal dimension of the landscape, that is, one half of the ridge-to-stream distance in that landscape. Average ridge-to-stream distance varies considerably among different landscapes but is fairly consistent within a particular physiographic province. Fels and Matson (1996) obtained estimates of ridge-tostream distance for various physiographic provinces by visualizing digital terrain models within each region, measuring ridge-to-stream distance for a number of typical landforms, and taking the mean of these measurements to obtain a representative value.

Local elevation techniques ignore hydrological connectivity of the surface topography (Gallant and Wilson, 2000); they simply quantify the relative position of a point of interest compared to its surroundings.

\section{Surface flow}

Flow accumulation, Catchment Area (CA) and specific catchment area, beyond their hydrological purpose of use; provide measures to quantify relative terrain position across a landscape. Terrain position is hypothesized to be related with continuous grid of surface flow that accumulatively increases through upward to downward positions. Theoretically from each cell, all flow is passed to the lower neighbour with the steepest slope. Flow amount at the highest positions is zero or very near zero, moving downwards accumulation increases and at the channels and depressions accumulation reaches its highest values. Therefore a range of values between 0 to large positive values can be utilized to quantify relative terrain position. Catchment area is defined as area draining to catchment outlet and 
specific catchment area is similarly upslope area per unit width of contour (Moore et al., 1991). All land-surface parameters related to surface flow require a DEM that has been preprocessed to remove artifact depressions and flat areas.

Flow accumulation is the basis of other measures such as Catchment Area (CA) and specific catchment area, upslope contributing area, wetness index and extraction of streams and ridges. In its simplest form flow accumulation is the number of upslope cells that flow into each cell. It is calculated via flow algorithms e.g. D8, Rho8, FD8, FRHo8.

D8 (Deterministic eight node) algorithm is developed by O'Callaghan and Mark (1984). It is a single flow direction algorithm. From each cell, all flow is passed to the neighbour with the steepest downslope gradient in a $3 \times 3$ window resulting in 1 out of 8 possible drainage. D8 can model convergence i.e. several cells draining into one, but not divergence i.e. one cell draining into several cells (Gruber and Peckham, 2009). Rho8 (Random eight node) algorithm is developed by Fairfield and Leymarie (1991). It is a randomized single direction algorithm, an improved version of D8 and stochastic rather than deterministic. It prevents parallel flows to a degree and produces more realistic looking flow networks. FD8 and FRHo8 algorithms are multi-flow algorithms and differently allow divergence to be represented (Moore et al., 1993). Therefore algorithm highly eliminates parallel flows and is more proper to represent catchment area and upslope contributing area (Gallant and Wilson, 2000). For instance; Behrens et al. (2009) in their study computed catchment area based on the multi-flow algorithm. This variable is used both as a stand-alone attribute and as the basis to calculate relative hillslope position. However they did not provide criteria for describing terrain positions as they have run multivariate statistics on those data together with others.

Flow accumulation or other DTMs calculated out of it e.g. catchment area are continuous rasters with grid values of amount of flow passing through. Ideally on a smooth surface with no curvatures, flow accumulation increases gradually through downward positions (Figure 2.20 a). However terrain has concavities in plan where the flow runs into or convexities where it disperse (Figure $2.20 \mathrm{~b}$ ). Therefore those concavities in mid positions may gain large amount of flow accumulation which may end up with a bias in describing terrain position. 


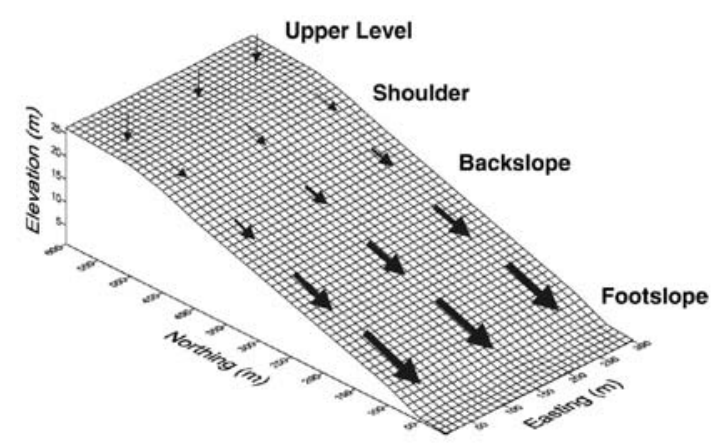

a

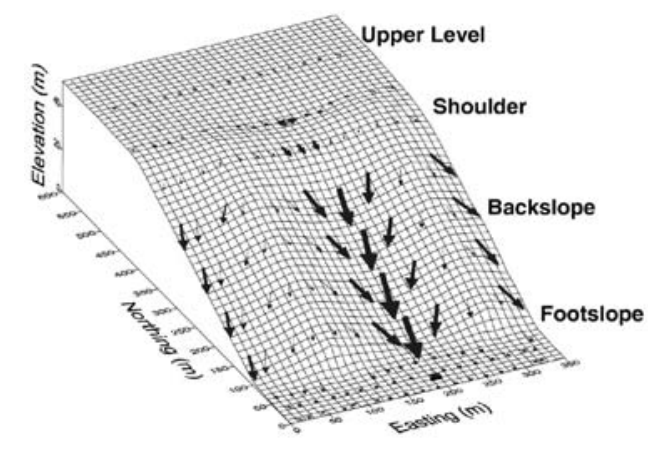

b

Figure 2.20: a) surface water flow patterns on a planar inclined surface b) surface water flow on a surface with significant plan curvature leading to convergence and divergence of flow paths (Pennock, 2003). Size of arrows indicate the flow accumulation at that point on the surface

Another shortcoming of surface flow based methods in describing terrain position is that accumulation might reach very high values at channels where as accumulation rules create main stream lines that are only one cell wide, and therefore large contrasts may arise in the flow accumulation computed for a cell on a main stream line and its neighbour (Burrough et al., 2000). This is not proper for use in describing relative terrain position, as we expect cells close to each other to have similar conditions, especially in the bottom of a valley.

\section{Proximity to terrain network}

Terrain network is comprised of streams and ridges that characterize the structure of the land surface. Those features so-called surface specific features constitute minima and maxima of surface, hence are used as a basis to describe position across terrain. Basically, proximity to those features quantifies the relative position of a location across the landscape.

Euclidean distances of a given location to the nearest streamline and ridgeline can be used to represent a relative position. Simple arithmetic operation via Euclidean distance from the identified ridges and the streams can approximate the slope position by dividing the distance from the nearest ridge by the sum of the stream distance and ridge distance:

$$
P i j=\frac{E D s}{E D s+E D r}
$$

For any cell $\mathrm{xij}, \mathrm{Pij}$ is the relative position index of that cell

Where;

EDs is the Euclidean distance to the nearest stream-line, and

EDr is the Euclidean distance to the nearest ridge-line. 
Index create a $0-100$ scale of the slope position, with 0 being the ridge and 100 the bottom of the slope.

Skidmore (1990) categorized landscape into five proportional classes namely; valley, lower mid-slope, mid-slope, upper mid-slope, ridge based on above index. Hall (2008) proposed a three-class scheme; high (ridge), medium (side slope) and low slope positions according to the same index.

Euclidian distance is straight line distance measured on xy plane and it produces a distance raster. The main problem of this approach is that the Euclidean distance is often not sufficient to represent topographic characteristics (Shi, 2005). For example morphometry is assumed smooth and it weakly respect topographic structure, e.g. any point is assumed in between a ridge and stream line however this may not always be the case, the nearest stream can be in between point and the ridge, then local topographic context is not truly represented. Especially where the ridge lines and stream lines converge, index values are not reliable.

\section{Compound approaches}

Compound approaches are based on indices that incorporate more than one of the measures of relative position in an arithmetic operation. Some of them are mentioned below;

Relative Hill Position (RHP); a compound index is based on elevation difference between the elevation of a local pixel and the elevation of its closest stream pixel. After delineating the streamline from the DEM, the pixel values of the stream are replaced by the original elevation values of the DEM. Afterwards, a nearest neighbour approach is used to assign the elevation on the streamline to other non-stream pixels. This elevation data layer is referred to as streamline elevation data layer (sEL). Finally, the streamline DEM is subtracted from the original DEM, resulting in a grid containing local elevation (e.g., MacMillan et al., 2000; Behrens, 2003).

$\mathrm{RHP}=((E L-s E L) /(c E L-s E L)) \times 100$

Where;

$\mathrm{EL}$ is the original elevation

sEL streamline elevation

cEL is crest elevation 
Another method is subtracting the CA of the original DEM and the CA calculated over an inverted DEM (Behrens, 2003). Thus, in mid slope positions the values are close to zero. In upper slopes the values are negative, whereas in toe slopes the resulting values are positive.

Compound indices are more sophisticated versions of above methods however they suffer from same weaknesses, and measures gathered are not easily described as they do not directly refer to a specific characteristic of terrain.

\subsection{Terrain Network}

There are broadly three approaches of producing terrain networks which were mentioned in section 2.2.3.2.2. They are; topologic approach, hydrographic approach and morphometric approach. In this section terrain network extraction methods will be limited for hydrographic approach since the other two approaches are not relevant methods of extracting terrain networks for this study. The reason why topologic approach is eliminated is; it is a graph based approach and it is rather interested in the topological relations between peaks, pits, saddles that are represented with nodes and ridge and channel lines that are connecting them. Spatial properties are not important and number of links and their degree of connectedness is described (Wood, 1996). Therefore ridge and channel lines that connect specific points do not necessarily regard topography. Terrain topology methods can be referred to for constructing connectivity relationship of specific points; i.e. peaks and ridges for this study. Morphometric approach is already employed for the study for gathering morphometric forms of Dikau (1989) where specific features of terrain network are included as well, i.e. peaks, ridges, channels. However morphometric forms lack connectivity and they are areal units rather than linear and they portray form rather than structure.

\section{Hydrographic Approach}

Extracting stream lines and drainage patterns is a standard procedure of watershed and surface flow analysis that is mentioned in section 2.3.2.2.1 Terrain Position under 'Surface Flow'.

Hydrographic approaches rely on two basic DTMs called flow direction and flow accumulation. Flow accumulation can be used to extract stream network as well. When flow accumulation per pixel exceeds a minimum threshold, those pixels are considered as streams / flow paths. Streams can be later put into a hierarchy of orders of magnitude e.g. Strahler; (1964), Shreve, (1966) (Figure 2.21) and Horton (1945). 


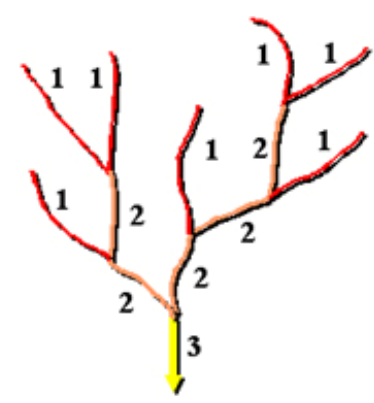

Strahler

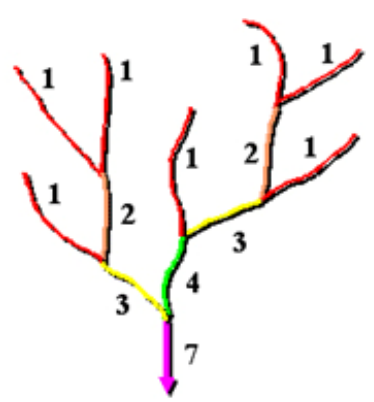

Shreve

Figure 2.21: Magnitudes of Strahler and Sheve stream orders.

Basins and watershed are similarly extracted based on surface flow algorithms. The Watershed process addresses the influence of terrain on surface water hydrology by modeling the movement of water over the land surface. Watersheds are derived out of flow direction and flow accumulation. From these intermediate results, stream network and the boundaries between watersheds the areas drained by particular stream systems are extracted. Watersheds can be further subdivided into basins associated with particular branches of the stream network. A watershed process yields watershed boundaries representing areas of land that catch rain or snow and drain to specific pour point to streams, and in addition smaller basins. Number of those basins can be adjusted by branch and basin parameters of a given analysis system. Watershed/basins cover the whole area with connected polygons.

This particular structure of watershed is not very appropriate for directly adopting them as terrain network. Because they have a polygonal topology but not a network, and basin polygons need to be closed and their boundaries might coincide even with river beds. There are also ridge extraction algorithms but they usually produce disconnected ridges. One interesting option to extract ridges even in orders of magnitude is to utilize inverse DEM as input and apply stream extraction procedure.

Existing algorithms applied on DEMs can be implemented without modification on an inverted DEM. An inverted DEM can be obtained by subtracting the DEM from a constant value larger than the highest elevation of the DEM. This simple arithmetic operation invert DEM values such that new maximum value gets the minimum value of the original DEM and visa versa. Peaks in the surface become sinks when the DEM it is inverted and in this case these sinks are genuine termination points and must not be filled (Gallant and Wilson, 
2000). There are some formal use of this method, e.g. calculating dispersal area that is downslope equivalent of catchment area (Gallant and Wilson, 2000).

\subsection{Object-based Image Analysis}

Object-based image analysis (OBIA) is a relatively novel approach in digital image analysis and classification. According to widely accepted definition of OBIA proposed by Hay and Castilla (2006); “Object- Based Image Analysis (OBIA) is a sub-discipline of GIScience devoted to partitioning remote sensing imagery into meaningful image objects, and assessing their characteristics through spatial, spectral and temporal scale". At its most fundamental level, OBIA performs image segmentation, attribution, classification and it has the ability to query and link individual objects (Hay and Castilla, 2006). Segmentation of images dates back to mid 1970s (Haralick et al., 1973), however analysis tools for OBIA have became available in software packages and studies have become widespread only for more than a decade.

The term OBIA however is recently being considered to cover wide range of application area, relating to other fields such as biomedical imaging (Hay and Castilla, 2008). A new term "GEOBIA" (Geographic Object-based Image Analysis) has emerged to be used for geospatial object-based image analysis. GEOBIA is now declared formally in the literature (eg. Blaschke, 2010), moreover, every 2-year symposium since 2004 being held under the name OBIA has shifted its name into GEOBIA for 2010.

First earth observation satellites came along with pixels typically coarser than or similar to size of the objects of interest and per-pixel techniques were capable of handling domain specific image analysis. With the increasing spatial resolution, alternative methods to represent objects that are made up of a group of pixels have necessitated. The rationale behind is that classic per-pixel classification approaches do not explicitly make use of the spatial characteristics inherent within the image (Blaschke and Strobl, 2001) and it is intended to produce image objects that match objects on the ground (Woodcock and Harward, 1992).

Object-based Image Analysis (OBIA) was primarily established for remote sensing practices. DEM and DTM data are represented with on a regular grid matrix that is very similar to that of satellite imagery. More over terrain data in nature is continuous and per- 
pixel methods had several insufficiencies in handling them e.g. per-pixel classes do not relate to individual landform elements and yield scattered classes across the scene. For a decade, OBIA being explored in terrain classification applications (e.g. Miliaresis and Argialas; 1999; MacMillan et al., 2000; Miliaresis, 2001; Strobl, 2001; Blaschke and Strobl, 2003; Drăguț and Blaschke, 2006) and proved to yield promising results.

OBIA at its most fundamental level involves segmentation, once the objects are produced they inherent information from pixels which contain far more information than individual pixels do, and can be later classified regarding object information and interlinkages of objects.

\subsubsection{Segmentation}

Segmentation of the image into discrete meaningful objects constitutes a key step in objectbased image analysis and classification.

Segmentation however is a loosely defined term. It generally refers to process of dividing an image into smaller regions having homogeneous colour, texture, or both (Stepinbsky et al., 2006), and in the literature especially older, it is sometimes used synonymously as classification. Segmentation however lies somewhere in between classification and regionalization (Strobl, 2008), where classification is based on spectral similarity and regionalization is delineation based on not necessarily similarity but other functional properties or significant edges. Segmentation is rather based on contiguity criterion. This produces relatively homogeneous areas bounded by lines where the homogeneity is disrupted (Minar and Evans, 2008), so that similar, neighbouring pixels are grouped into discrete image objects such that some measure of variance within the object does not exceed a specified threshold.

There is vast amount of image segmentation algorithms developed. Meinel and Neubert (2004) according to an application-oriented quality test performed on seven segmentation algorithms widely used, reported that region growing based algorithm of eCognition yield the best results. The procedure is based on 'Fractal Net Evolution Algorithm' (FNEA) approach which is an efficient method to describe semantics within largely self constructing and dynamic networks. It combines fractal structure of the world and semantics with object 
orientation (Baatz and Schape, 2000). FNEA is already embedded in a commercial software 'eCognition' of Definiens Inc.

FNEA is an iterative heuristic optimization procedure that is developed to get the lowest possible overall heterogeneity across an image. The algorithm is an iterative local optimization which performs pair wise merging of pixels that minimizes the average heterogeneity within image objects (Figure 2.22).

First a homogeneity criterion is defined for the segmentation. For a d-dimensional feature space the heterogeneity h (or degree of fitting as named by Baatz and Schape, 2000) is described as:

$\mathrm{h}=\sqrt{\sum_{d}\left(f_{1 d}-f_{2 d}\right)^{2}}$

where;

$\mathrm{f}$ is any object feature that is used to determine heterogeneity between objects.

Heterogeneity/homogeneity criterion has both spectral and a spatial components. Spectral homogeneity is defined by the spectral values of the pixels belonging to the object; spectral values can be weighted by arbitrary by spectral band weights. The spatial homogeneity component is based on the deviation of the object's shape from a compact and a smooth shape. Compactness is defined as the ratio of the perimeter of the object and the square root of its area (the number of pixels it contains), and smoothness is defined as the ratio of the object's perimeter and the length of its bounding box (parallel to the image borders) (Feitosa et al., 2005).

Objects grow from single pixels and are merged pair vise to neighbouring objects. In each processing step an object can be merged together with the neighbouring object/objects that provide the smallest growth of global heterogeneity. The basis for growing is the degree of difference between two regions. If the difference is small, two regions are likely to merge. In the FNEA, these differences are optimized in a heuristic process by comparing the attributes of the regions in a pair wise fashion, if they the objects do not exceed heterogeneity criterion defined by scale they merge into one object and process iterates until the heterogeneity criterion is reached (Figure 2.23). 


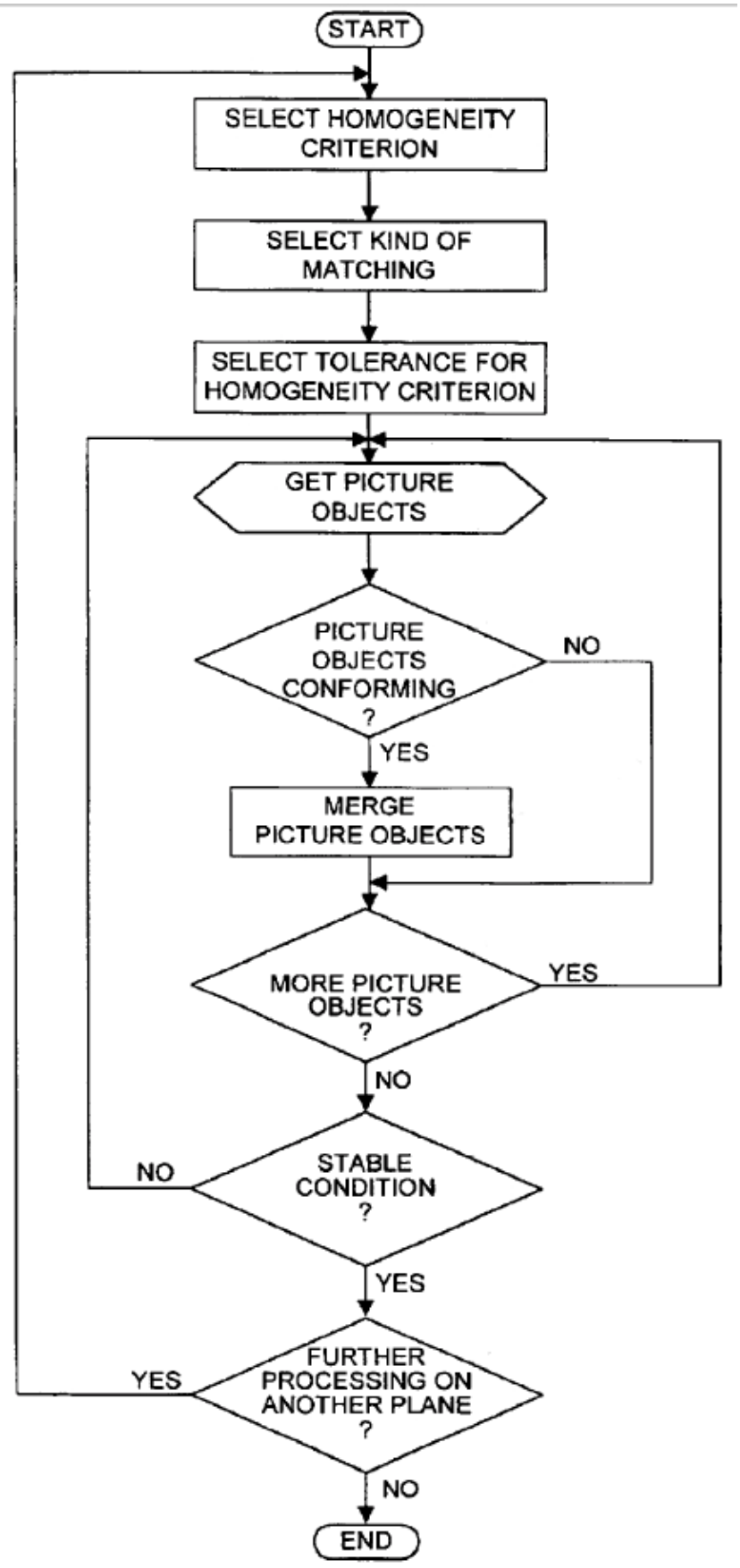

Figure 2.22: Flowchart of segmentation based on Fractal Net Evaluation Algorithm by Baatz and Schape (2004). 


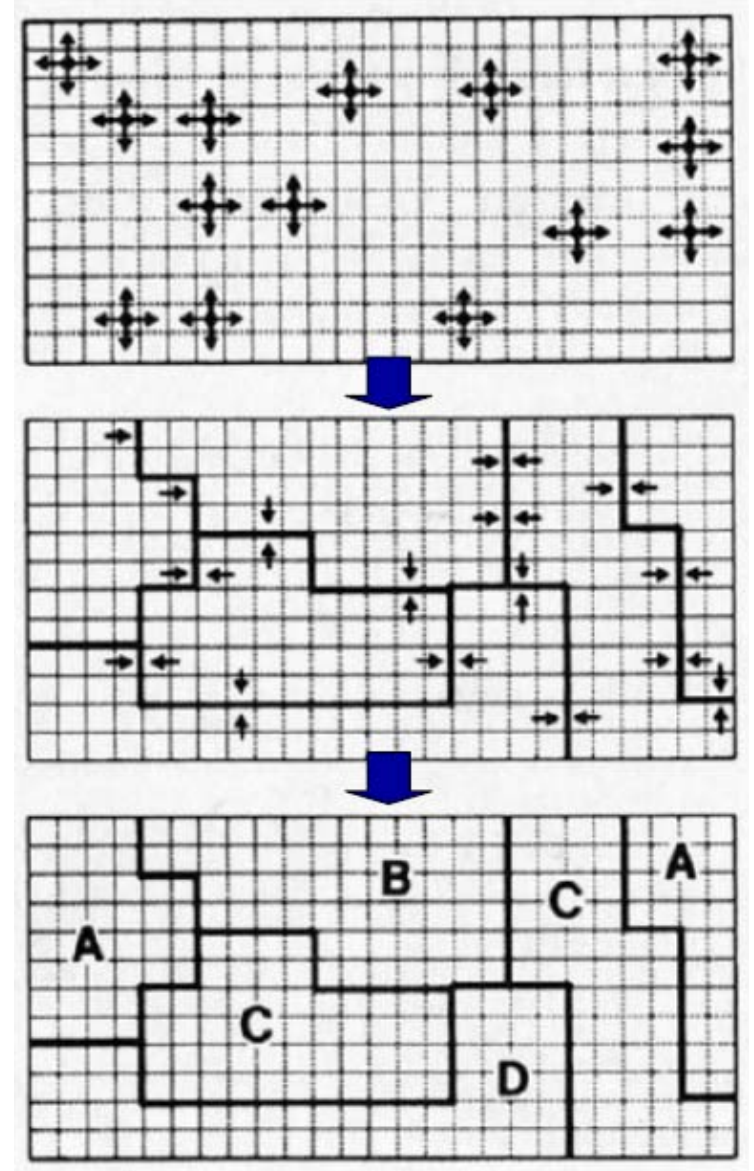

Figure 2.23: Segmentation of seed pixels into objects at a particular scale.

Performance of the object-based image analysis depends essentially on the segmentation quality, and that depends on segmentation algorithm and the segmentation parameter settings. Homogeneity criteria are defined by the user. Segmentation parameter set for FNEA based segmentation comprises basically of scale, spectral and spatial heterogeneity.

The degree of heterogeneity within an image object is controlled by a subjective unitless measure called the 'scale parameter'. Scale parameter is related with level of segmentation or size or image objects, it is set similarly as a threshold value such that some variance within the object does not exceed it. If this value is small one would end up with fine segments, if it is large, then the image objects will be larger as the heterogeneity is set to a high value for each object. Figure 2.24 represents the effect of scale for a given image layer at three different scales. 

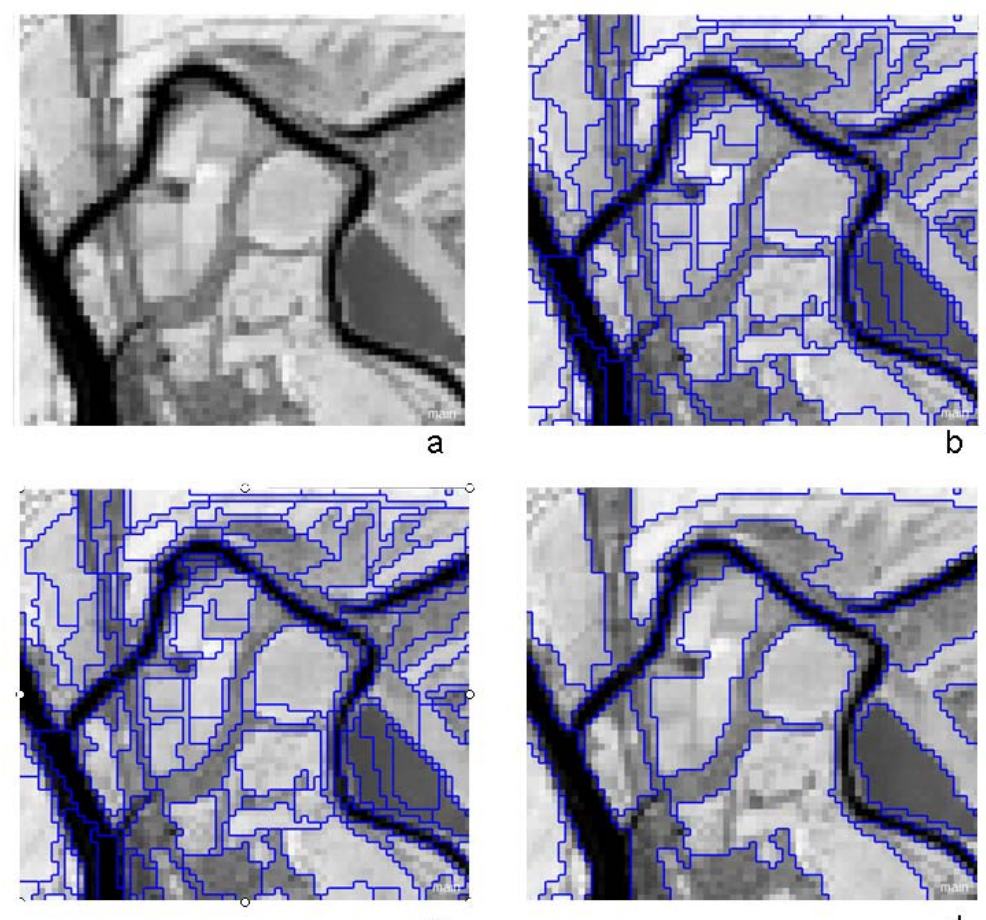

C

d

Figure 2.24: Segmentation with increasing scale parameter on a subset of satellite band a) original band b) segmentation at scale: 5 c) segmentation at scale: 10 segmentation at scale: 20 .

However, theoretical framework of FNEA is not provided and users have to find useful segmentation levels in a 'trial and error' style (Blaschke and Hay, 2001; Burnett and Blaschke, 2003; Walker and Blaschke, 2008). According to Addink et al. (2007), optimal scale of observation for a given objective would depend on: ii) the spatial heterogeneity of the landscape, and ii) the spatial and temporal extent and frequency of the phenomenon of interest and the processes responsible for it.

Some authors have exercised of optimal levels of segmentation that maximize accuracy of results with regard to a single response variable. Addink et al. (2007) found different levels of segmentation produced the most accurate results for estimates of leaf-area index and biomass. Luscier et al. (2006), using a manual, iterative process, identified the segmentation level that resulted in objects that gave the highest coefficients of agreement between field plot photos and control data. Kim and Madden (2006) used variance of pixel spectral values within an image object to determine the level of segmentation that would give the highest accuracy for classifying forest types. 
Mentioned approaches set their scale parameters based on a quality measure that is computed by comparing the outcome produced by the segmentation algorithm with an available reference result. Feitosa et al. (2005) however proposed a genetic algorithm that determines optimal segmentation parameters. Genetic approaches typically do not require any explicit model of the underlying process and can work with virtually any fitness function. Dragut et al. (2010) developed a technique for estimating the scale parameter in image segmentation of remotely sensed data using an estimation function called Estimation of Scale Parameter (ESP) that builds on idea of local variance (LV) originally described by Woodcock and Strahler (1987). ESP can be loaded as a tool in eCognition software.

A single optimal segmentation level is usually inefficient in representing a scene of interest. It is very likely that there are a hierarchy of patterns and image information in different scales and these can be simultaneously represented via multi-scale segmentation. The challenge and flexibility of the multi-scale segmentation/object relationship modelling approach lies in the proper definition of the semantic rules which relate the lower level landscape units to higher levels of organization (Burnett and Blaschke, 2003).

FNEA typically segment images simultaneously at both fine and coarse scales, then building image semantics between levels and their elements. Multi-level segmentation is implemented in either in bottom-up or top-bottom approach. Initial procedure is segmentation; pixels should be first shifted from pixel domain to object domain (Figure 2.25).

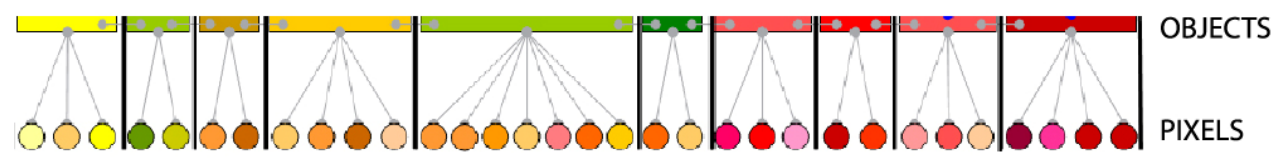

Figure 2.25: Pixels are merged into objects given a homogeneity/heterogeneity criteria defined by scale.

An image object is a group of homogeneous pixels that provide information about the area it represents. Moreover, every image object is linked to its neighbours and to its sub-objects and super objects if there is a multi-level hierarchy of object levels. Together the image objects construct a network, which enables access to the contextual information. 
The principal challenge and flexibility of this multi-scale approach lies in defining the aggregation rules for the lower level entities, which result in improved image classifications, and a new framework for integrating semantic rules in image processing.

Multi-scale segmentation: Segmentation can be performed on several scales with different scale parameters. This produces a set of object layers within a hierarchical network. This procedure is constrained so that boundaries of objects in one level fits into objects of other levels enabling consistency of sub-objects and super-objects and their relationships in the classification step. Segmentation of the images at fine and coarse scales is important in the object-based multi-scale analysis in order to extract boundaries of the dominant objects occurring at the corresponding scales (Hall et al., 2004). In the case of FNEA in eCognition, the user determines the processes of interest within the scene and generates the corresponding levels of segmentation forming a network of horizontally (neighbour) and vertically (upper and lower) connected objects (Figure 2.26).

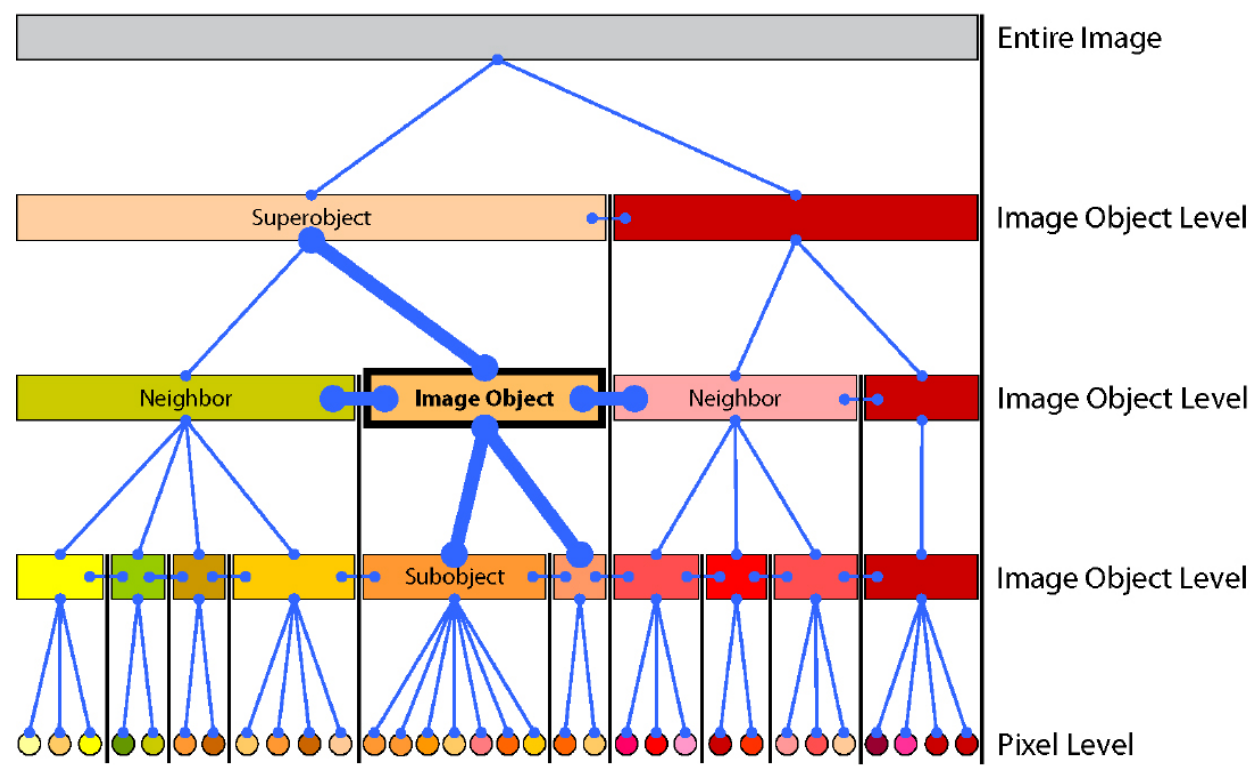

Figure 2.26: A typical multi-level hierarchical network of objects with interrelationships and sublevel, super-level relationships.

Image objects are interrelated and interrelated so that each object knows its context (neighborhood), its super-object and its sub-objects. And they have spatial consistency as they share common boundaries. 


\subsubsection{Classification}

Classification stage comes after segmentation and object attributes based on attribute statistics, shape, topology, and hierarchy are used by crisp or fuzzy rule sets to construct features representing real world entities from the basic segmentation results (Strobl, 2008).

For a fuzzy classification there are basically two ways of producing classes with membership values for each class; they are 'similarity relation model' and 'semantic import (SI) model' (MacMillan et al., 2000; Fisher, 2004). Objects can be either classified according to one of them. In SI model, each image object is compared to the defined membership functions and assigned to the class which produces the highest membership value for the object (Figure 2.7a a) Similarity relation model performs a multivariate fuzzy classification via a training set to define its 'class central concepts'. Each image object is compared to the training samples given and assigigned to the class the sample of which is the nearest in the feature space (Figure $2.7 \mathrm{~b}$ )

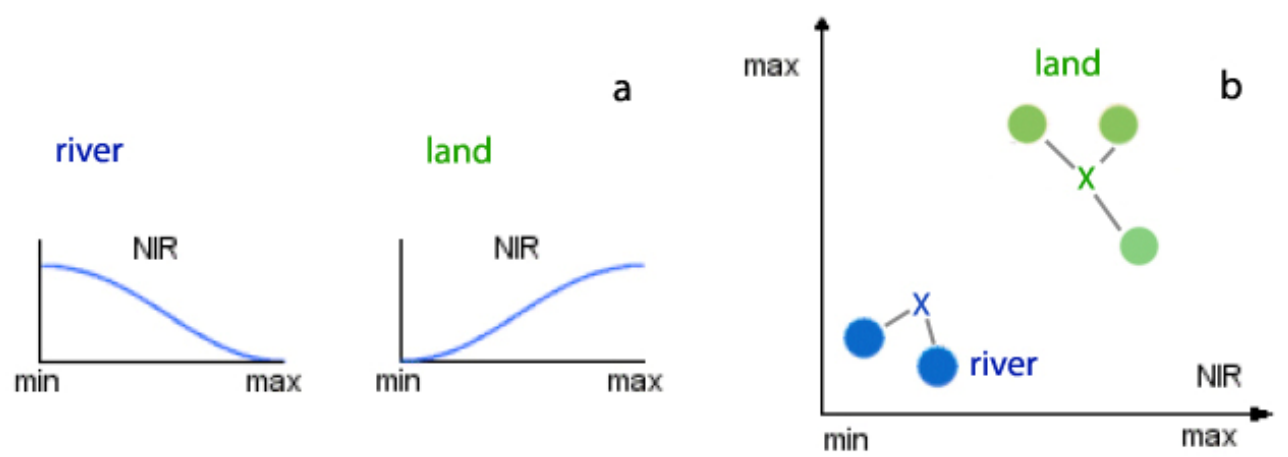

Figure 2.27 Description of classification rules a) in Sementic Import (SI) Model, b) in similarity relation model via training samples.

Either a 'similarity relation' or 'SI model' is employed; classification procedure makes use of object properties so-called 'features' for classification. Image objects contain far more information than individual pixels do. For instance; mean vale of a band or DTM within the object or shape of the object, adjacency to a particular class object are all examples for 'features' that constitute the basis for formulation of conditions for classification or further segmentation steps. It is crucial to find the right feature and the right threshold for conditions to yield better classification results. 
Taking eCognition as reference, an exceedingly wide range of features is provided to be used for classifying objects into classes. These features can be very broadly categorised into object-related features, and class-related features as it is described in eCognition tutorial (Definiens Developer 7, ReferenceBook, 2010).

\section{Object-related features:}

Object features are obtained by evaluating image objects themselves as well as their embedding in the image object hierarchy.

Layer values: These are features concerning the pixel channel values of an image object (spectral Features)., e.g.; mean values, brightness, standard deviation

Shape: With these features, the shape of an image object can be described using the object itself or its sub-objects, e.g., area, length, compact ratio, elongation

Texture: Texture features evaluate the texture of an Image object based on its sub-objects.

Hierarchy: These features provide information about an embedded image object within the image object hierarchy.

Thematic attributes: These are attributes of the thematic layer objects. This type of feature is only available if such a thematic layer has been imported into the project.

Customized Features: New features can be calculated in the customized feature dialog.

\section{Class-Related Features:}

Class-related features refer to the classification of other image objects that are taken into account for the classification of the image object in question.

Relations to neighbour objects: These features refer to existing class assignments of image objects on the same level in the image object hierarchy, e.g.; relative border to neighbours, distance to a certain class, and number of neighbours.

Relations to sub-objects: These features refer to existing class assignments of image objects on a lower level in the image object hierarchy, e.g.; number of sub objects of a specific class.

Relations to super-objects: These features refer to existing class assignments of image objects on a higher level in the image object

Relations to Classification: These features refer to the actual classification of an object. Example: The object is currently classified as what class, class name, and membership to a class.

Customized Features: New features can be calculated in the customized feature dialog. 


\section{CHAPTER 3}

\section{METHOD OF LANDFORM CLASSIFICATION}

In this study, an object-based classification method based on local geometry and geomorphometric context to produce landforms that represent general geomorphometry of the landscape is proposed. A 'morphometric classification' where DTMs that characterize form is employed, subsequently 'morphometric classes' and geomorphometric context that is represented with 'relative terrain position' and 'terrain network' are reclassified through a set of logical operators. Accordingly; morphometric classes are reorganized regarding geomorphometry of the landscape.

Some basics of the classification prior to analysis are defined in the next section. Method will be briefly described in section 3.2, and in section 3.3, data set used for the study will be introduced. Case areas are described in chapter 4. Procedures for implementing the study are described in more detail in chapter 5 and chapter 6 .

\subsection{Classification Basics:}

\subsubsection{Scale}

Study is implemented at a scale range that corresponds to 'Mesorelief A' that is; a scale range coarser than microrelief as defined by Dikau (1989). Landscape scale, hillslope scale, microrelief and mesorelief are commonly referred to when describing the phenomenon or an analysis scale. Hillslope scale corresponds to microrelief (Schmidt and Hewitt, 2004). Landscape scale corresponds to a wider range that is in between microrelief and mesorelief (Dragut and Blaschke, 2006). Description of these scale terms can be found in section 2.2.4.1 (Phenomeana Scale). Accordingly, is very likely that most of the significant physical and biophysical processes that act on the earth surface takes place at microrelief to 
mesorelief, particularly 'Mesorelief A' as defined by Dikau (1989). Therefore scale range 'Mesorelief A' is adopted as the analysis scale for this study.

Furthermore, characteristic scale (Wood, 2009) is examined for the case areas and some measures helped in defining particular scales for the analysis. Scale is managed by window size that is determined to calculate DTMs. DEMs of $15 \times 15 \mathrm{~m}$ resolution are generated and window sizes that are considered to represent particular scales are utilized to produce input DTMs as described in section 5.1 (DEM generation) and 5.2 (DTMs derivation) .

$1 / 25000$ scaled topographic contours with $10 \mathrm{~m}$ vertical interval are employed for the analysis. This data is quite appropriate to make a classification on Mesorelief A (described range covers topographic maps of 1/5000 to $1 / 50000$ (See Table 2.5). Moreover, 1/25 000 scaled topographic maps are the standard maps that cover the whole country and they are widely available.

\subsubsection{Classes}

'Form element' model of Dikau (1989) (Figure 2.2) which is very appropriate for an exhaustive 'general' geomorphometric classification of a surface constitutes the basis of classification for this study. This scheme describes elementary forms with all possible combinations of slope and curvature and efficiently describes both sloping; e.g. spurs, hollows and non-sloping; e.g. ridges, planes of the terrain. There are 15 form elements including 9 sloping and 6 non-sloping forms. This scheme however regards local geometry only, hence can be adapted to any surface, even an artificial objects' surface. Therefore classes gathered pertaining to this scheme is better labeled as, $\mathrm{XX}, \mathrm{XY}$, etc. (Figure 2.2), i.e. $\mathrm{X}$ for concavity, $\mathrm{Y}$ for convexity instead of ridge, spur, etc. or called 'peak like feature'. Form elements alone may not properly represent land surface hence they should be associated with geomorphometric context before being addressed as a landform class. Classification scheme involves 15 landforms namely; peak, ridge, saddle, plain, channel, pit, nose, spur, spur foot, shoulder slope, planar slope, foot slope, hollow shoulder, hollow, hollow foot. Some good representatives of those classes have been captured from map scenes with topographic contours of $10 \mathrm{~m}$ vertically interval overlaid on shaded relief of a hilly terrain for $1 \times 1 \mathrm{~km}$ sample areas First group of features (Figure 3.1) pertain to nonsloping part of the land surface and second group of features pertain to the sloping part (Figure 3.2). 

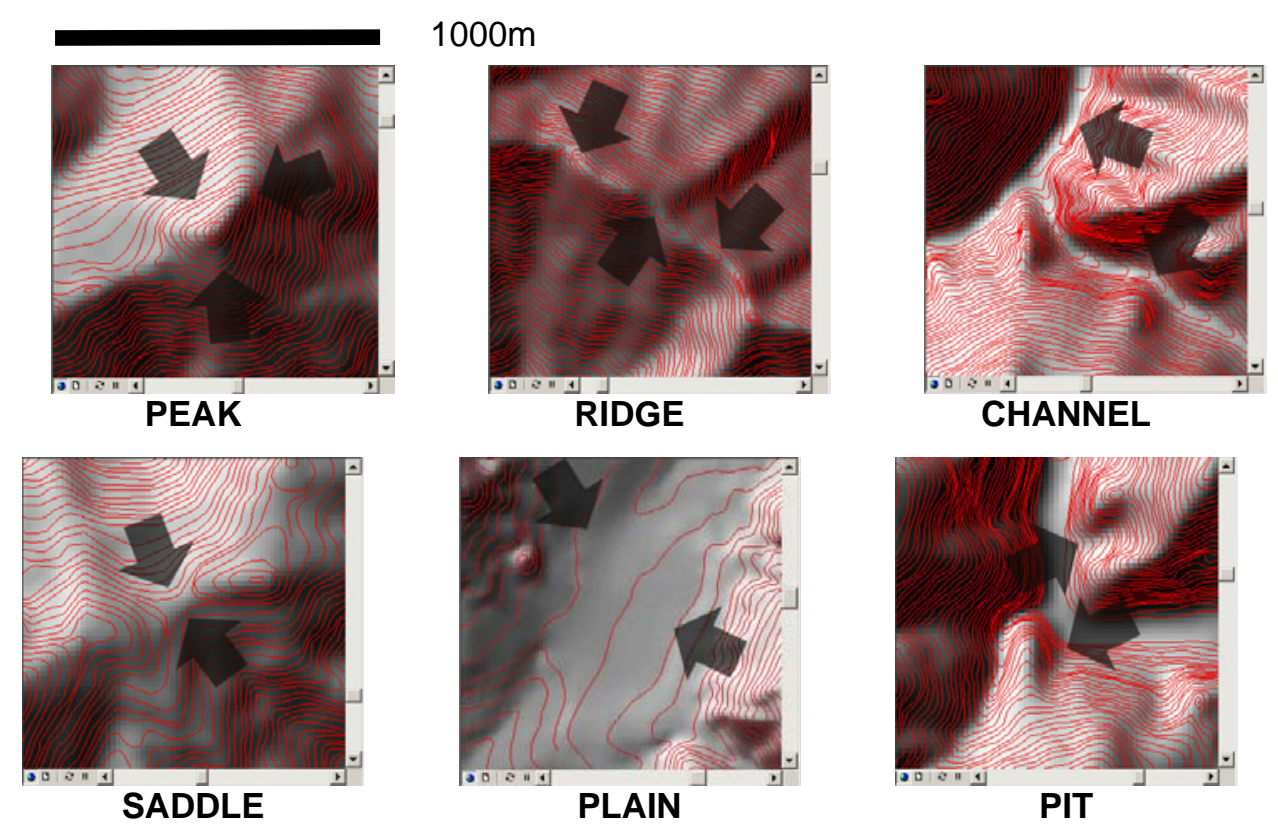

Figure 3.1: Representative features that pertain to non-sloping part of the land surface
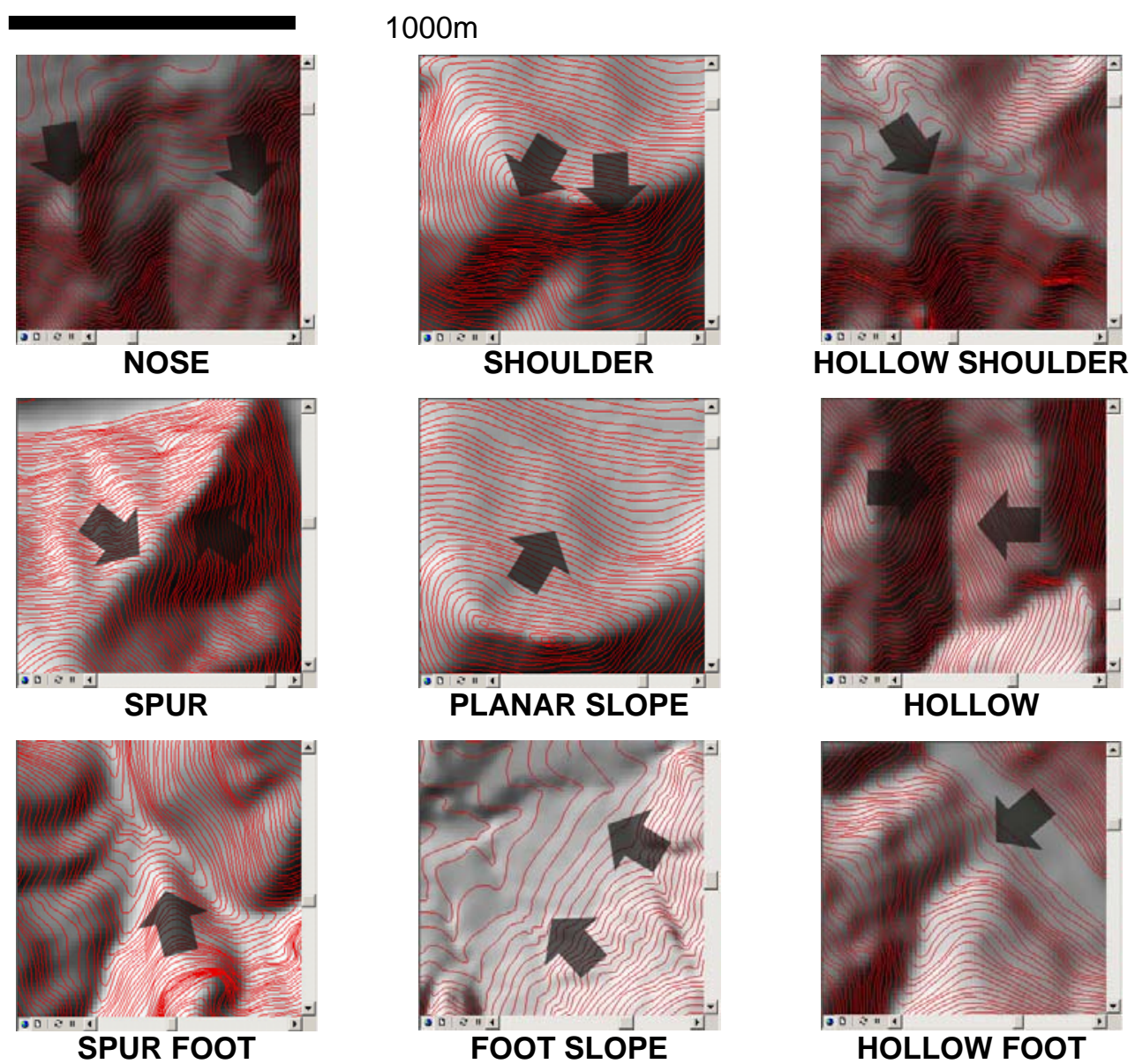

Figure 3.2: Representative features that pertain to non-sloping part of the land surface 


\subsection{Description of the method}

Flowchart for the study (Figure 3.3) provides basic steps in implementing the proposed study where morphometric classification and 'geomorphometric classification (highlighted with texture) constitutes the main part.

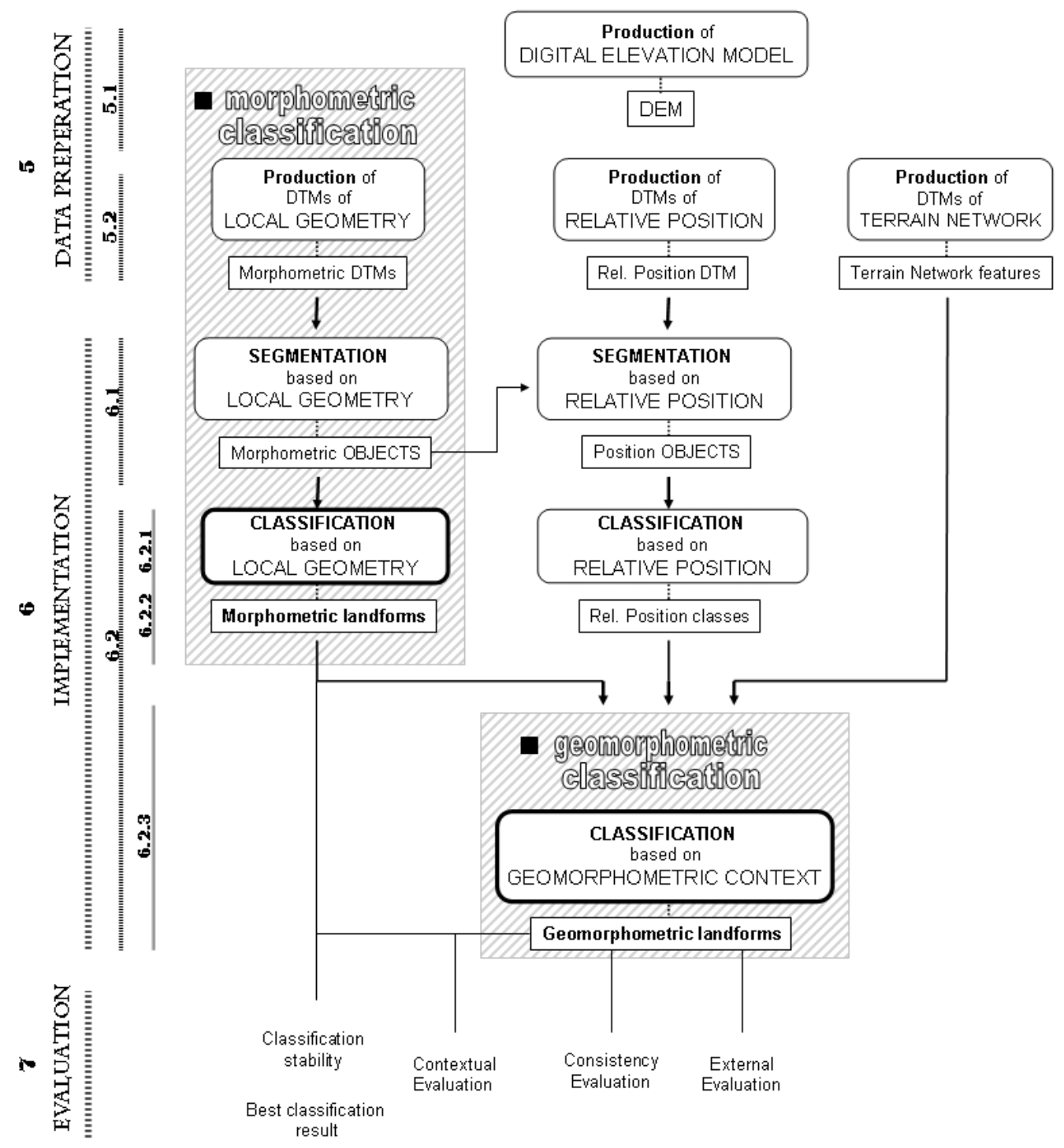

Figure 3.3: Flowchart of the proposed landform classification method.

Numbers on the left side of the flowchart are chapter and section numbers where the relevant analyses are described. 
Accordingly; classification based on local geometry / morphometric classification is performed, then classification based on geomorphometric context / geomorphometric classification is implemented. A minor classification step to be mentioned is terrain position classification prior to geomorphometric classification.

Morphometric classification: A section from the main flowchart (Figure 3.3) is given to describe the steps of morphometric classification (Figure 3.4).

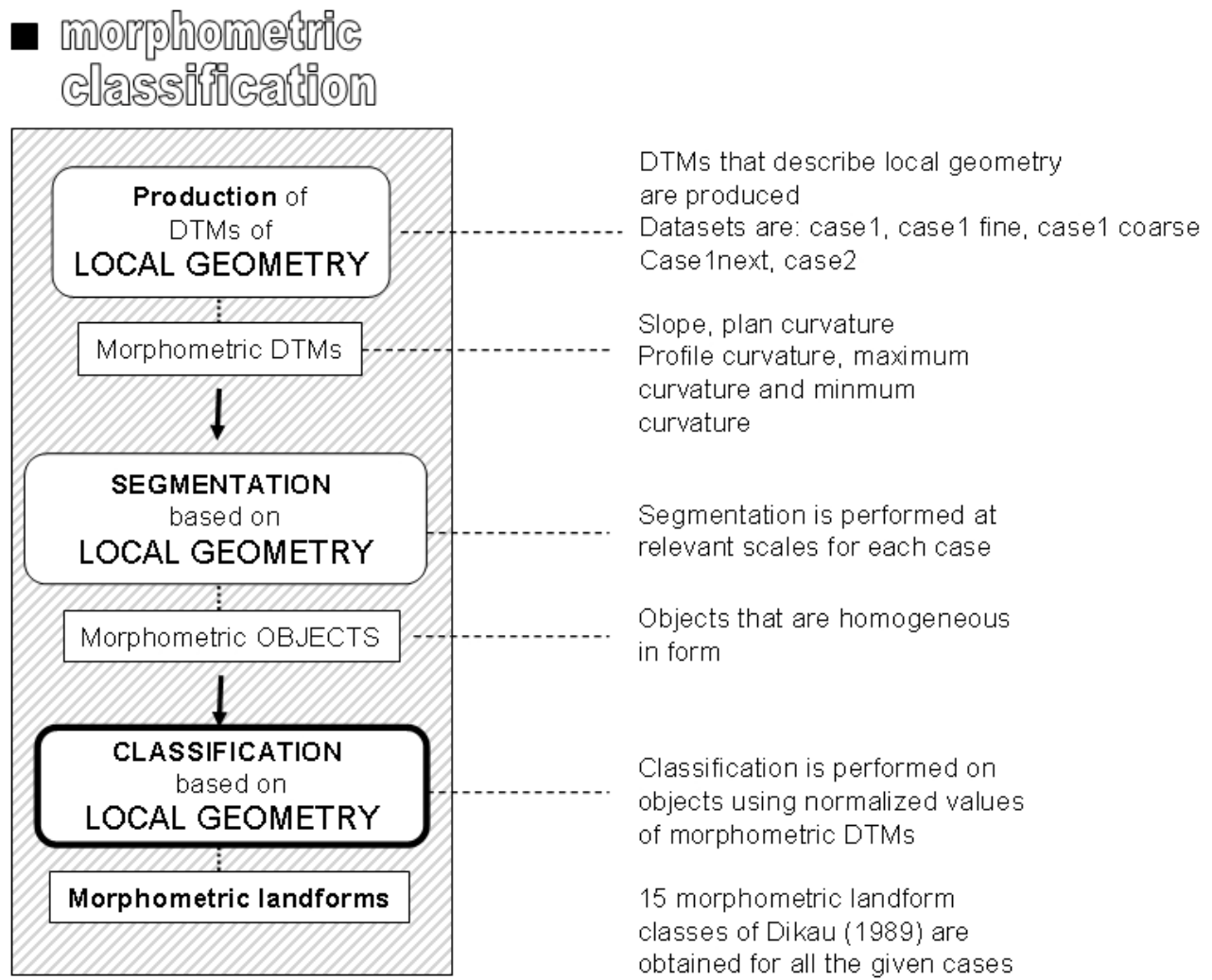

Figure 3.4: A section from the main flowchart which describes classification based on local geometry /morphometric classification

A set of DTMs that represent the local geometry of the terrain are used as input for morphometric classification. Before classification, DTMs are transformed from pixel domain into object domain to overcome weaknesses of pixel-based analysis in landform classification. A fuzzy classification to represent continuous nature of landform classes is employed. Among the two methods of implementing fuzzy classification ('similarity relation model' and 'semantic import (SI) model; SI is preferred. Because; 'similarity relation model' requires central concept to be defined by means of a sample set that trains multivariate classification. Landforms of general geomorphometry are considered as 
prototype objects (see section 2.2.2: Landform ontology) where a central concept in a landform class is described by the minimum or maximum levels of a particular characteristic i.e. convexity, concavity rather than any interval within a data range, similarly as in remote sensing imagery. SI model on the other side permits employing flexible semantic descriptions like high, low, lowest, directly into image classification. Therefore SI model is a more proper way of translating the conceptual model into classification and permits formal recognition and incorporation of the imprecise and overlapping semantics used to describe or classify data. Form objects are classified through a fuzzy SI model and 15 fuzzy morphometric classes are obtained.

Terrain position classification: Terrain position constitutes a minor part of the classification and its steps are given in Figure 3.5 which is a section from main flowchart. DTM that represent relative terrain position is produced, and then a higher level segmentation is created out of morphometric segmentation. Morphometric objects are duplicated into a higher level and objects are classified using relative position as spectral information. As a consequence, boundaries come from the morphometric segmentation and spectral values are utilized from terrain position index: TPI. There are three classes of terrain position, 'upper', 'mid' and 'lower'.

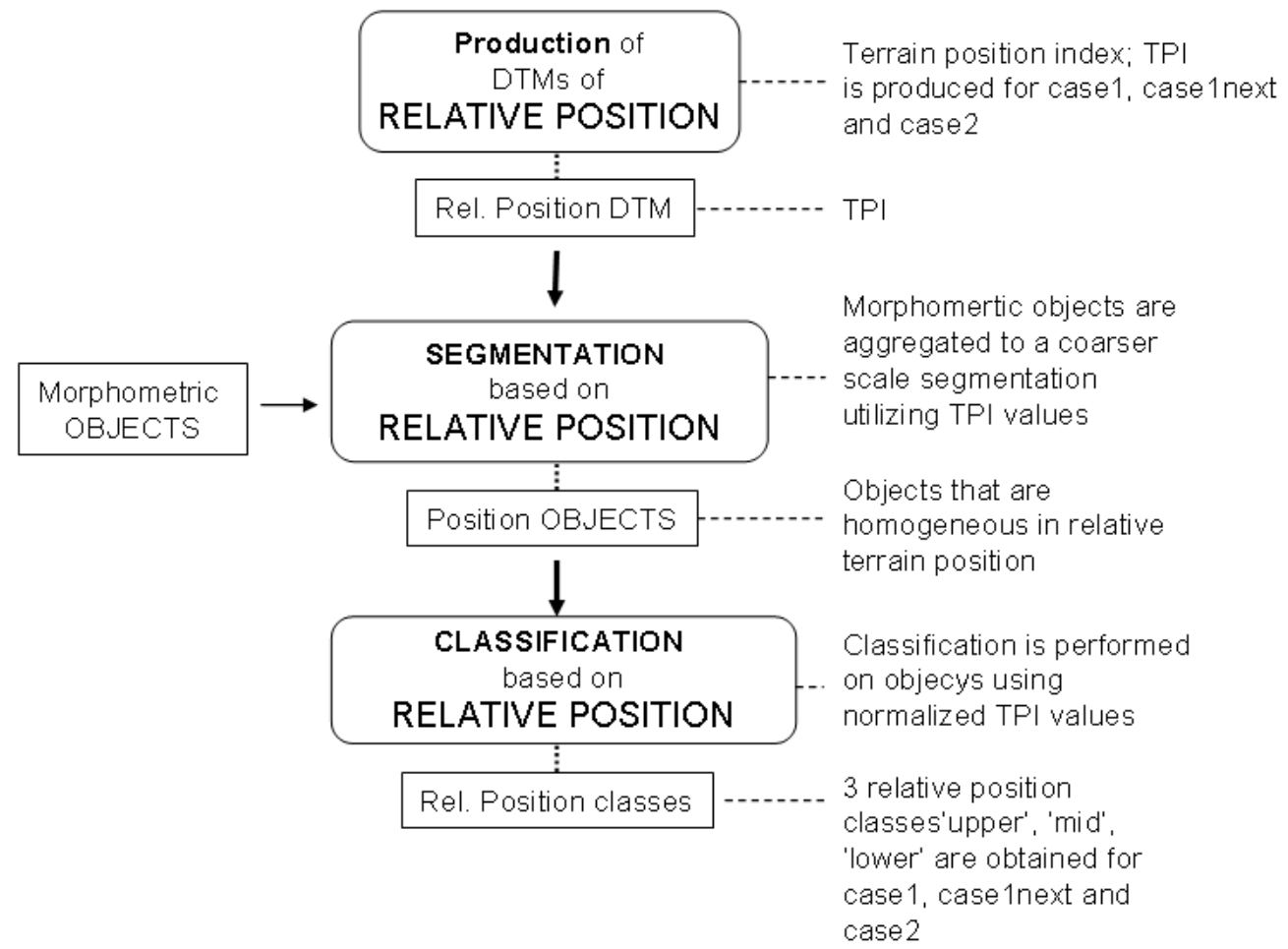

Figure 3.5: A section from the main flowchart which describes terrain position classification 
Other set of DTMs that represent geomorphometric context are structural linear features; 'streams' and 'ridges' which constitutes terrain network. They are extracted out of DEM. Higher orders of magnitudes in the hierarchy (Strahler, 1964) are utilized directly as thematic inputs into geomorphometric classification.

Geomorphometric classification: Geomorphometric classification employs three input information (Figure 3.6). Morphometric landforms obtained from morphometric classification at the lowest level, position categories at a higher level, streams and ridges as independent thematic linear features were all covered in a multi-level hierarchy of objects in eCognition. Geomorphometric classification is actually a reclassification of form elements based on logical rules regarding typical organization of landforms across the landscape. Form elements this way are reorganized to portray their formal relative position across the landscape. Form elements are checked and for instance a form element that is not expected to be observed at a particular position is reclassified into its proper landform class. Structural information; streams and ridges were utilized to determine specific features; ridge, peak and channels, as these forms are very likely to coincide with surface specific features.

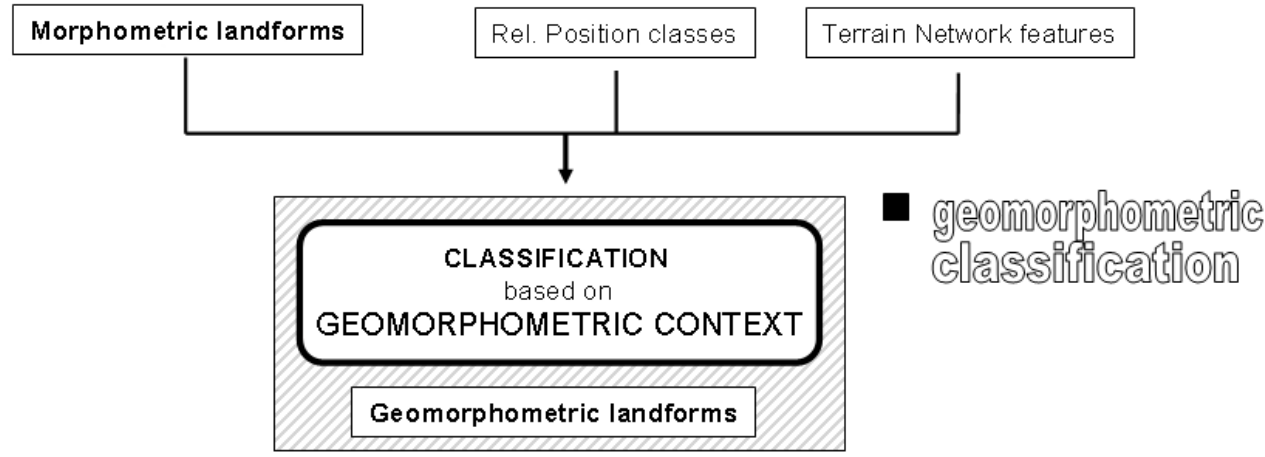

Figure 3.6: A section from the main flowchart which describes geomorphometric classification

Proposed method of classification is applied to two case areas. These are referred to as 'case1' and 'case2' throughout this study. 'Case1' is a mountainous area with dissected valleys. 'Case2' is relatively smoother area with some dominating hills. An adjacent area with 'case1' that has an overlapping part with 'case1' is also studied to evaluate the stability of the classification and this area is referred to as 'case1next' (Figure 3.7). An additional case that is convincingly flat is also exercised. 

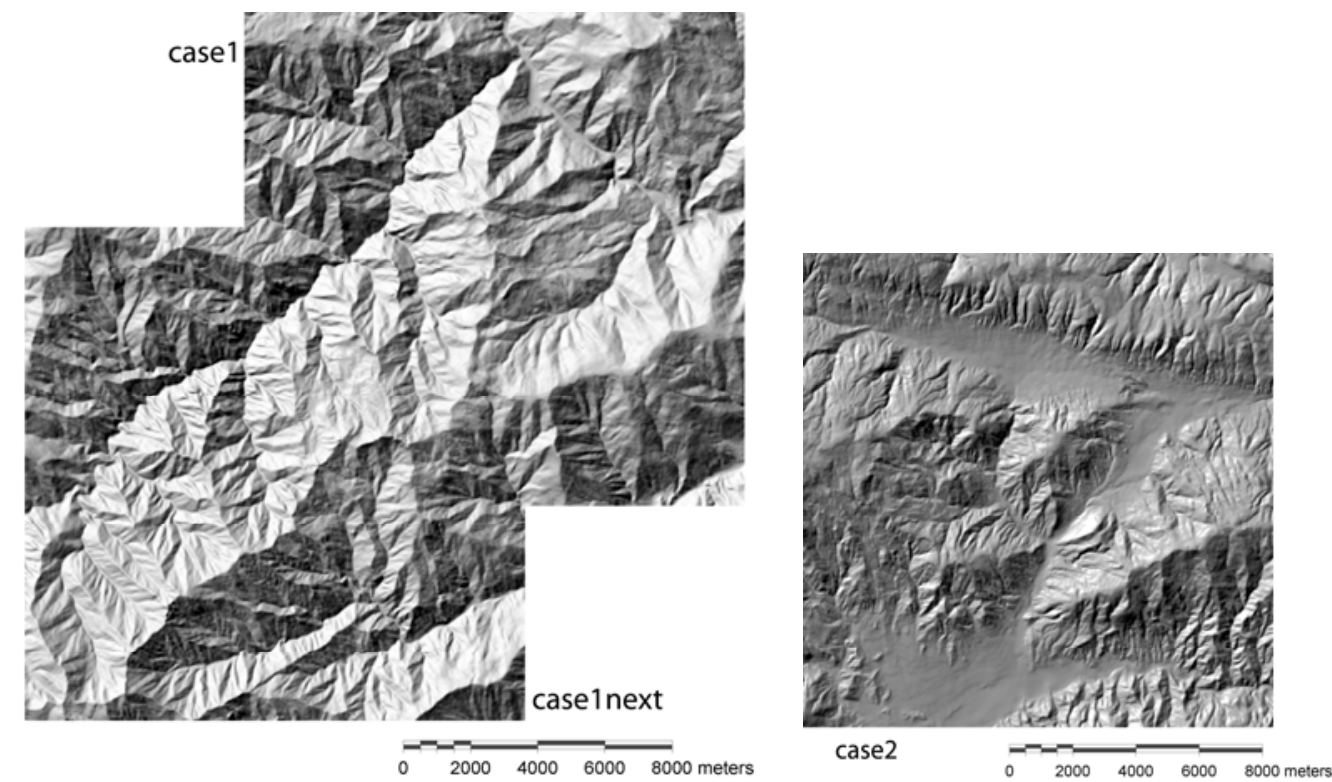

Figure 3.7: Shaded reliefs of the study areas 'case1', 'case1next' (left) and 'case2' (right)

Geomorphometric classification that is proposed for this study was intended to be exercised for two areas that have different characteristics of topography. Then it was considered some additional classification exercise will add value to the research, for instance; applying classification method on an overlapping area to test if the results are stable, applying the method to different scales of the same area. Multi-scale classification is however exercised for 'morphometric classification' only. Different scales of dataset pertain to a finer and a coarser scale of 'case1' and they are referred to as 'case1 fine' and 'caselcoarse' throughout this study. Method is also applied at a convincingly flat topography to test if the it works for a flat topography where some of the classes of Dikau (1989) do not necessarily exist. As a result different classifications were evaluated on different datasets and case areas using exactly the same classification method, that is keeping all thresholds and all classification rules the same. These classification attempts and their intention are listed (Table 3.1).

\subsection{Dataset}

DTMs generated out of DEMs are utilized for classification analysis. A set of DTMs are produced for each case area. They can be broadly categorized into two as:

(i) Morphometric DTMs,

(ii) Geomorphometric DTMs

- terrain position DTM

- terrain network DTMs 
Morphometric DTMs represent local geometry of land. Terrain position and terrain network DTMs represent geomorphometric context.

Table 3.1: List of different case studies, their intention of being exericised, classification method employed and their relevant scales as DTM window size and data sets

\begin{tabular}{|c|c|c|c|c|}
\hline Case & intention & classification & $\begin{array}{l}\text { Scale } \\
\text { (window size) }\end{array}$ & dataset \\
\hline $\begin{array}{l}\text { Case1 } \\
\text { Case2 }\end{array}$ & $\begin{array}{l}\text { To test if the } \\
\text { method works on } \\
\text { different case } \\
\text { areas with } \\
\text { different } \\
\text { topography }\end{array}$ & Geomorphometric & $\begin{array}{l}45 \times 45 \\
(675 \times 675 \mathrm{~m})\end{array}$ & $\begin{array}{l}\text { Morphometric } \\
\text { DTMs, } \\
\text { Geomorphpmetric } \\
\text { DTMs }\end{array}$ \\
\hline $\begin{array}{l}\text { Case } 1 \text { fine } \\
\text { Case } 1 \\
\text { Case 1 coarse }\end{array}$ & $\begin{array}{l}\text { To test if the } \\
\text { method works on } \\
\text { different scales }\end{array}$ & Morphometric & $\begin{array}{l}15 \times 15,45 \times 45, \\
75 \times 75 \\
(225 \times 225 \mathrm{~m}, \\
675 \times 675 \mathrm{~m}, \\
1125 \times 1125 \mathrm{~m})\end{array}$ & $\begin{array}{l}\text { Morphometric } \\
\text { DTMs }\end{array}$ \\
\hline $\begin{array}{l}\text { Case1 } \\
\text { Case1next }\end{array}$ & $\begin{array}{l}\text { To test } \\
\text { classification } \\
\text { stability on an } \\
\text { overlapping area }\end{array}$ & Geomorphometric & $\begin{array}{l}45 \times 45 \\
(675 \times 675 \mathrm{~m})\end{array}$ & $\begin{array}{l}\text { Morphometric } \\
\text { DTMs } \\
\text { Geomorphometric } \\
\text { DTMs }\end{array}$ \\
\hline Case 3 & $\begin{array}{l}\text { To test if the } \\
\text { method works on } \\
\text { a very flat } \\
\text { topography }\end{array}$ & Geomorphometric & $\begin{array}{l}45 \times 45 \\
(675 \times 675 \mathrm{~m})\end{array}$ & $\begin{array}{l}\text { Morphometric } \\
\text { DTMs } \\
\text { Geomorphometric } \\
\text { DTMs }\end{array}$ \\
\hline
\end{tabular}

\section{DEM}

Topographic contour data from $1 / 5000$ to $1 / 50000$ is quite appropriate to generate DEMs and DTMs at the scale range adopted for this study that is Mesorelief A (Dikau, 1989). DEM at resolution $15 \times 15 \mathrm{~m}$ is found to be relevant to portray similarities vs. differences of landscape form and structure optimally. Moreover topographic data is widely available and has standards. Therefore, this study is implemented on $15 \mathrm{~m}$ DEMs generated out of digital contour data sets of $10 \mathrm{~m}$ vertical interval and further used as input for deriving DTMs.

\section{DTMs}

DTMs are derived out of DEMs of $15 \mathrm{~m}$ resolution and constitute the source data to implement landform classification. DTMs used in the study can be grouped broadly into two as mentioned in previous chapters as, morphometric and geomorphometric where geomorphometric DTMs are subdivided into two as 'terrain position' and 'terrain network'.

Morphometric DTMs: Morphometric DTMs represent local geometry of the surface even outside a land surface. Slope and curvature parameters are used to describe the form of the terrain. (Dikau, 1989) proposes the use of slope and four curvature parameters namely; plan, profile, minimum, maximum curvatures to categorize terrain into landform classes. 
Complete set of these parameters are adopted to describe form of the terrain effectively both for the sloping and non sloping pieces of land.

DTMs that represent local geometry is derived using quadratic polynomials that are commonly and efficiently used in deriving first and second order derivatives; slope, curvature respectively out of DEMs. This method applies a quadratic approximation on a set of pixels within a window, and size of the window for calculating the derivatives is strongly related with the level of detail, thus scale of the classification product. Therefore, a series of evaluations were made to determine an appropriate window size that better represent landforms within a specific scale range.

Appropriate scale is compromised after a set of evaluations on the data set that will be extended in Chapter 4, and window size of $675 \times 675 \mathrm{~m}(45 \times 45$ cells $)$ was found appropriate to implement the core part of the analysis. A coarser and a finer scale dataset produced using $225 \times 225 \mathrm{~m}(15 \times 15$ cells $)$ and $1125 \times 1125(75 \times 75)$ window sizes are also studied to understand the effect of scale on extracting morphometric landform classes.

Geomorphometric DTMs: Form elements have a relative positioning across the landscape and a structure of organisation. Therefore, those locally delimited landform elements need linkages to wider topographic context (Deng, 2007) that are relative terrain position and terrain network.

Terrain position: Form elements should be organized according to their relative position across the landscape using a terrain position indicator, e.g. class 'foot slope' beyond being a form element with a significant slope and concave profile curvature, it is placed relatively at lower positions of the landscape.

Topographic Position Index (TPI) by Jenness (2006) is used to determine relative position of a specific location across a given landscape. TPI calculates a position index from elevation grids given a window size, and provides a simple and repeatable method to classify the landscape into position and landform category using the TPI values. Given range of TPI values landscape is subdivided in to three as 'upper', 'mid' and 'lower' positions. Theoretical slope profile models based on soil-landscape modelling studies mentioned in section 2.3.2.2.1 (Terrain Position) were used as basis to reorganize form elements given their category of relative position. 
Terrain Network: Terrain network is important topological information that characterises a surface; basin divides, ridges, flow paths, point features like local maxima; i.e. peaks construct the skeleton of the terrain and some specific form elements coincide with those surface specific features, i.e., ridge, peak and channel.

In this study 'streams' and 'ridges' are extracted and utilized as structural elements in landform classification. Streams are derived from DEM using flow path algorithm that converts flow accumulation raster into connected flow path linear features attached with orders of magnitude; i.e. Strahler order (Strahler, 1964). Strahler order of 4 and more is assumed as significant channels and utilized in landform classification.

Ridges are derived using the same algorithm and parameters but on an "inversed DEM" that typically ends up with ridge lines rather than flow paths. Some minor modifications are made regarding watershed basin structure which is computationally more robust. Similarly as in streams; Strahler order of 4 and more are assumed as significant ridges. 


\section{CHAPTER 4}

\section{STUDY AREA}

Two case areas were selected from two different locations in northeast Turkey (Figure 4.1).

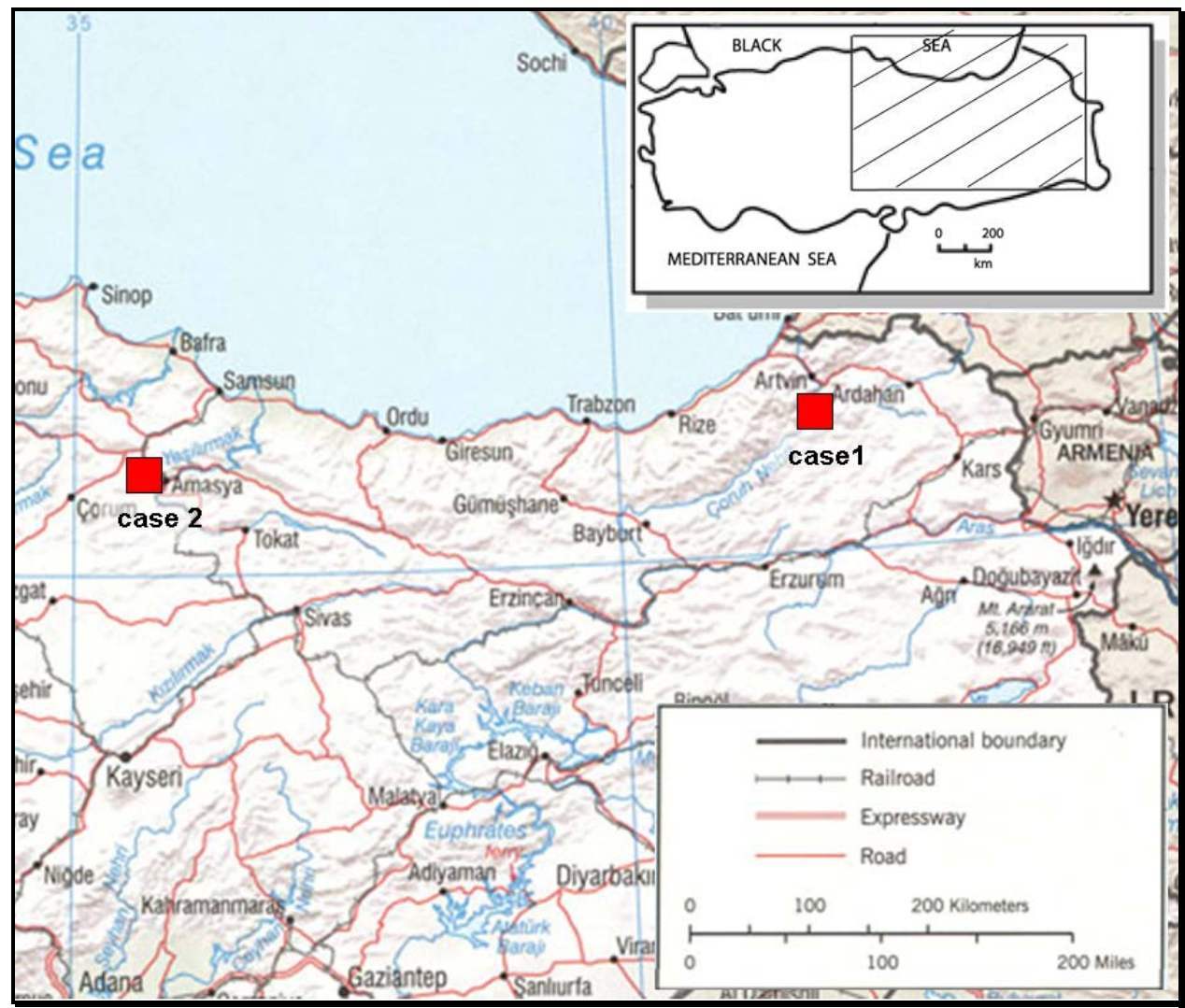

Figure 4.1: Location map of two case areas

'Case1' (Figure 4.2) has high relief, dissected valleys with steep hill slopes and sharp crests dominating the landscape where the geomorphology of the landscape can be dated to young stage. 


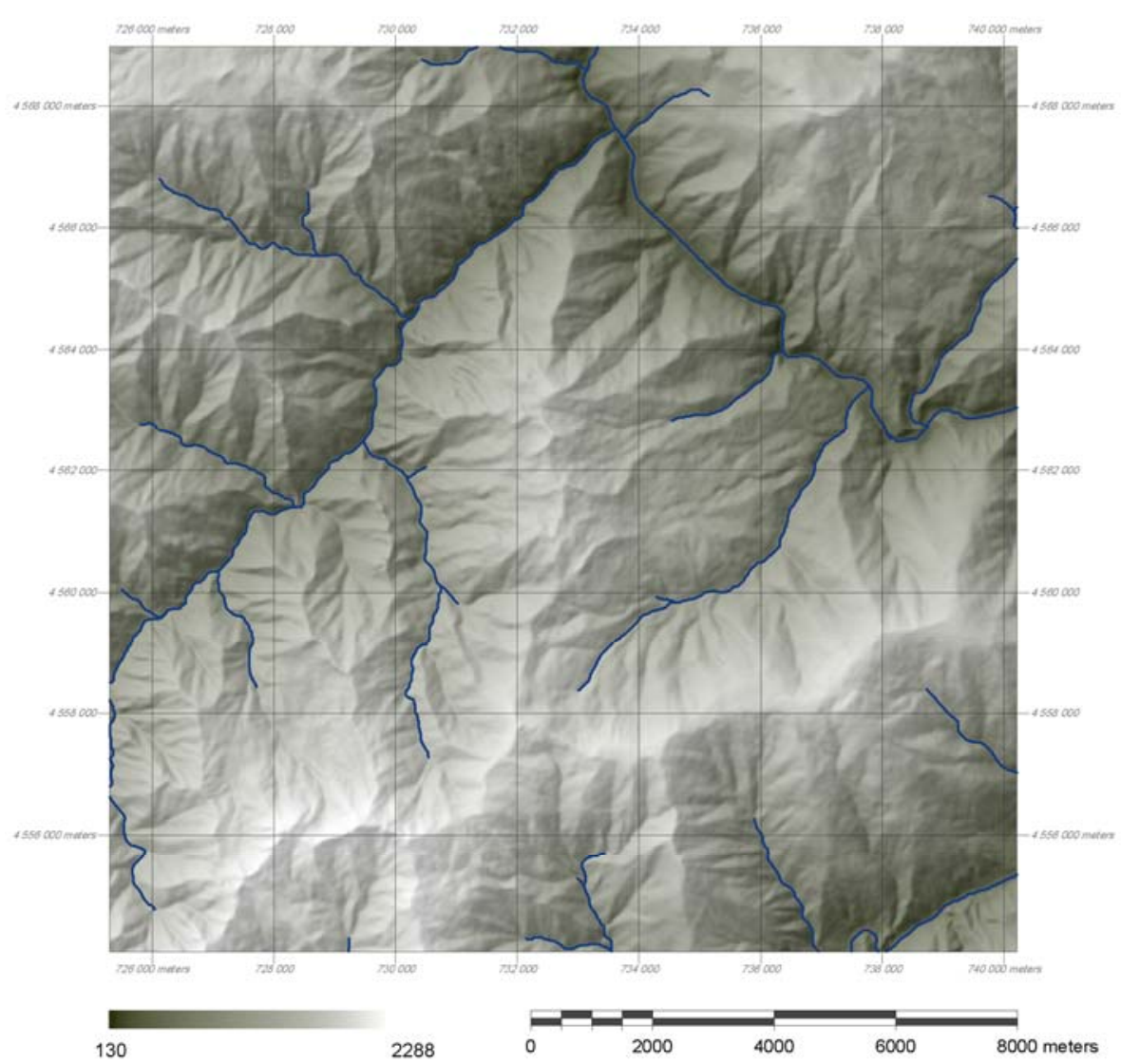

Figure 4.2: Study area: shaded relief map of 'case1'. For location see Figure 4.1

Where as in 'case2' (Figure 4.3) topography is smoother compared to 'case1', there are hills that dominate the landscape. There is a fault passing through east-west direction on the northern part of the study area and in the remaining part there are flat areas. Landscape seems to characterize geomorphologic conditions that can be dated to an older stage.

An additional case is tested for classification consistency and selected from an overlapping area with case1, so-called 'case1next'. It has similar characteristics with case1 as it is in the close vicinity (Figure (4.4).

Dataset for cases are originating from 1/25 000 topographical elevation contours of $10 \mathrm{~m}$ vertical interval, and they all have the same extents of $15 \times 15 \mathrm{~km}$. Case1 is in 'Kaçkar' mountains close to Artvin, and case 2 in is in Amasya. An additional case that is smooth and flat is also included for the evaluation of the method, however it is not illustrated in this chapter, it can be found at section 7.1.4 (Evaluation of classification on a flat topography) together with its classification result. Some information on location and extents of the case areas are listed in (Table 4.1). 


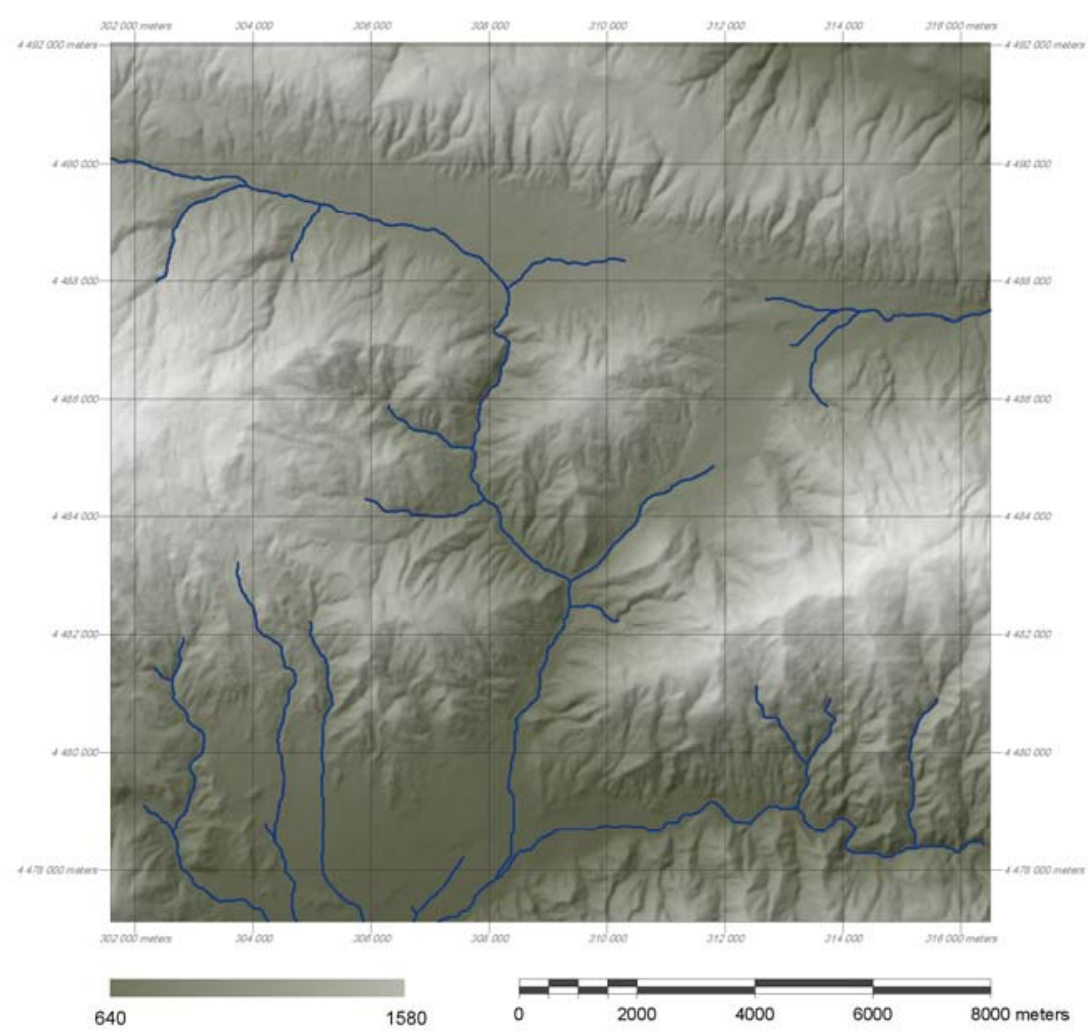

Figure 4.3: Study area: shaded relief map of 'case2'. For location see Figure 4.1

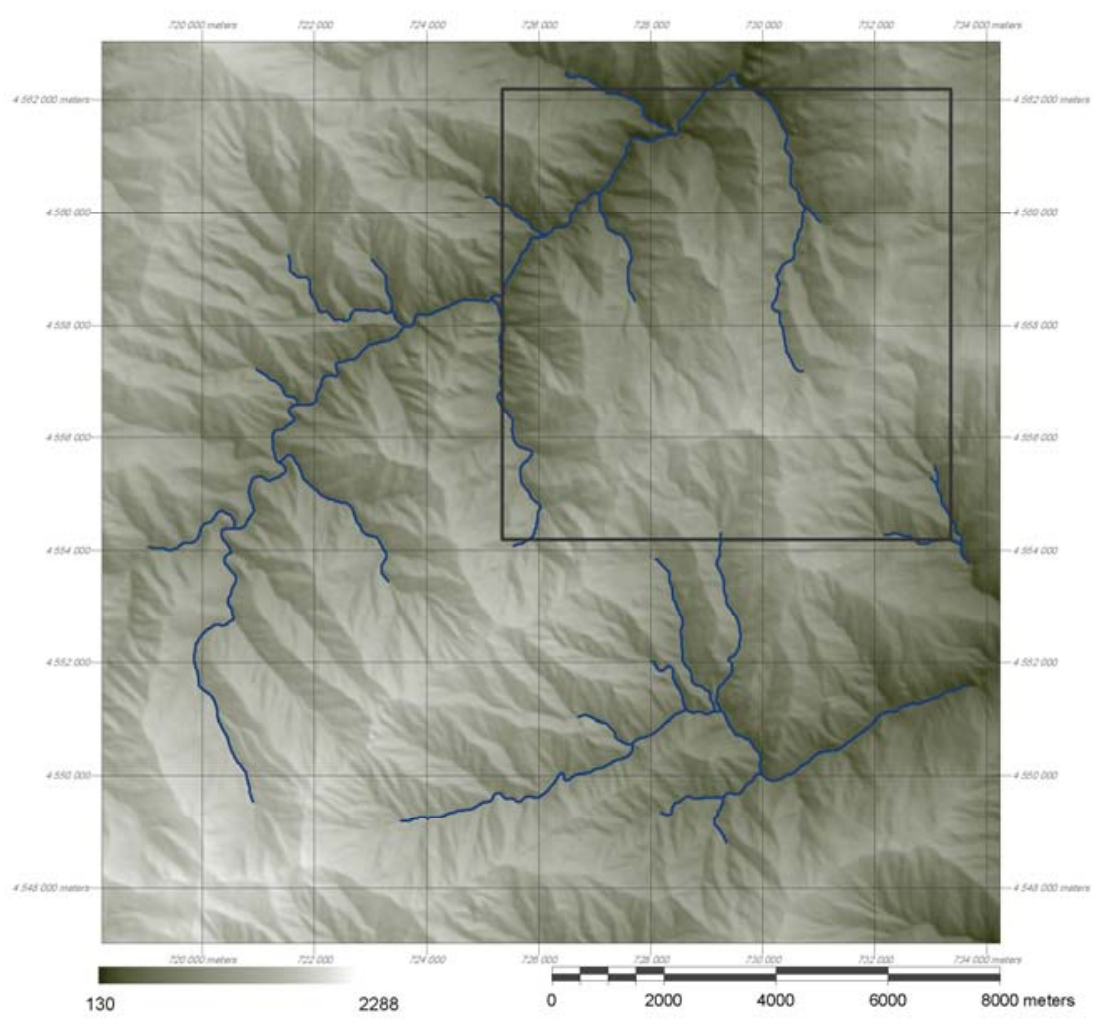

Figure 4.4:. Study area: shaded relief map of 'case1next' that overlap with 'Case1'. 8x8 km overlap to be studied in classification evaluation is shown 
Table 4.1: Vicinity, coordinates and extents of case areas: case1, case1next and case2

\begin{tabular}{lllll}
\hline Feature & Case1 & Case1next & Case2 & Case3 \\
\hline Dimensions & $15 \times 15 \mathrm{~km}$ & $15 \times 15 \mathrm{~km}$ & $15 \times 15 \mathrm{~km}$ & $15 \times 15 \mathrm{~km}$ \\
Area & $225 \mathrm{~km} 2$ & $225 \mathrm{~km} 2$ & $225 \mathrm{~km} 2$ & $225 \mathrm{~km} 2$ \\
& Top left & Top left $(718322 \mathrm{E}$, & Top left & Top left \\
& $(725297 \mathrm{E}$, & $4563005 \mathrm{~N})$ & $(301579 \mathrm{E}$, & $(424529 \mathrm{E}$, \\
UTM & $4568963 \mathrm{~N})$ & Bottom right & $4491991 \mathrm{~N})$ & $4305361 \mathrm{~N})$ \\
coordinates & Bottom right & $(734268 \mathrm{E}$, & Bottom right & Bottom right \\
of extents & $(740197 \mathrm{E}$, & $4547008 \mathrm{~N})$ & $(316528 \mathrm{E}$, & $(439428 \mathrm{E}$, \\
& $4554064 \mathrm{~N})$ & & $4477108 \mathrm{~N})$ & $4290467 \mathrm{~N})$ \\
Vicinity & Artvin, Turkey & Artvin, Turkey & Amasya, & Konya, Turkey \\
& & & Turkey &
\end{tabular}

Case areas: Case1, Case2 significantly portray classes proposed by Dikau (1989). However they are different in their morphology and landscape characteristics. Reason for selecting case 1 and case 2 as study area is basically to test validity of the method on two different topographies that portray proposed landform classes. Case3 however is a very flat topography significantly different from Case 1 and Case 2 it hardly portrays all landform classes adopted from Dikau's (1989) scheme, especially there are no or very few of sloping landforms as the area is dominated by flat plain. Criteria and parameters were organized in a manner to extract same conceptual classes of landforms on different data sets that have different data ranges given the same classification methodology and same parameters and sets of criteria. DTMs were derived using appropriate window sizes for each of the data set as mentioned in Section 5.2 (DTMs derivation)

Hypsometric curves can be employed to interpret the stage of a landscape in the cycle of erosion Strahler (1952) and to characterize landforms or the topography as well. Hypsometric curve represents altitude area relationship; it's a simple morphological index expressing the relative proportion of upland to lowland within a sample region. Altitude is plotted against proportion of area under that altitude. Hypsometric curves that belong to 'case1' and 'case2' areas portray the difference of general geomorphometry (Figure 4.5). Rapid decrease of relative area at high elevations is an indication that the area is mountainous with peaks and ridges.

Hypsometric integral has proven to be mathematically identical to "elevation relief ratio"; a topographic descriptor expressing the relative proportion of upland to lowland within a sample region. The elevation relief ratio $(\mathrm{E})$ is defined as: 


$$
\mathrm{E}=\frac{\text { mean }(\text { elevation })-\min (\text { elevation })}{\max (\text { elevation })-\min (\text { elevation })}
$$

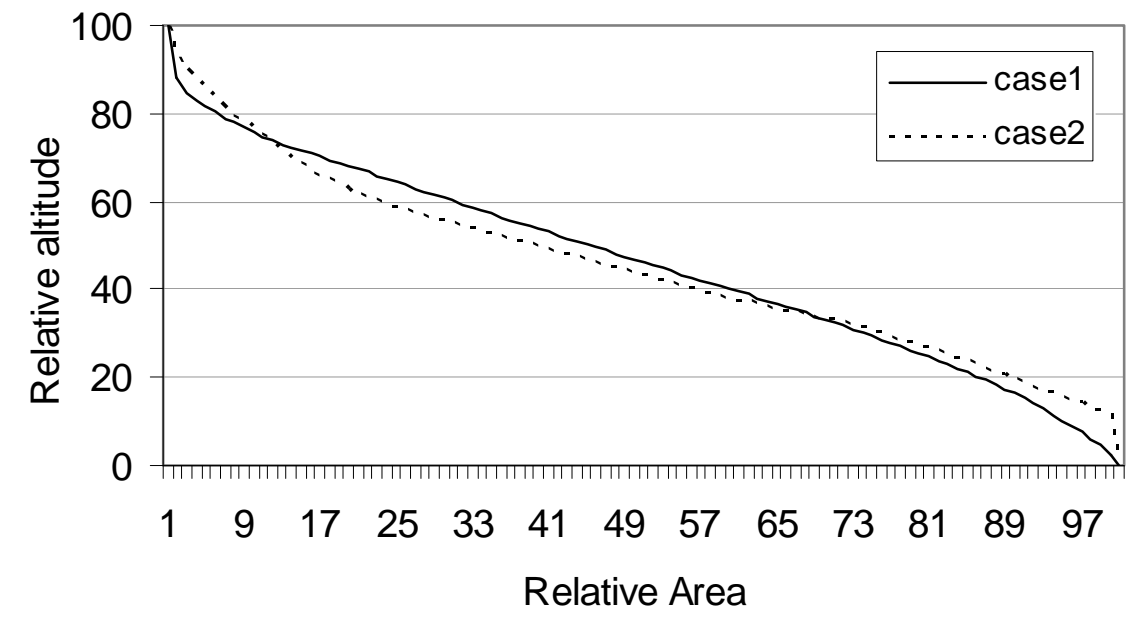

Figure 4.5: Hypsometric curves of two case areas: case 1, case2

E usually ranges from 0.15 to 0.85 , with values tending to cluster between 0.40 and 0.60 . Low values of $\mathrm{E}$ occur in terrains characterized by isolated relief features standing above extensive level surfaces, whereas high E values describe broad, somewhat level surfaces broken by occasional depressions (Pike and Wilson, 1971). 'E' of 'case1' is reasonably higher than that of 'case 2'. Data ranges for the two different cases also differ significantly e.g. slope is higher for 'case $1^{\prime}\left(0^{0}-41^{0}\right)$ with a mean value of $22.3^{0}$ compared to 'case $2^{\prime}$ ' $\left(0^{0}\right.$ $28.5^{\circ}$ ) with a mean value of $9.5^{\circ}$ and overall curvature is higher for 'case 1 ' i.e. maximum curvature fairly high compared to 'case 2' (Table 4.2).

Table 4.2: Summary statistics of case areas: case1, case1next and case2

\begin{tabular}{|c|c|c|c|c|}
\hline Feature & Case1 & Caselnext & Case 2 & Case 3 \\
\hline elevation & $\begin{array}{l}\text { min: } 130 \mathrm{~m} \\
\text { max: } 2288 \mathrm{~m} \\
\text { mean: } 1133 \mathrm{~m}\end{array}$ & $\begin{array}{l}\text { min: } 389 \mathrm{~m} \\
\text { max: } 3218 \mathrm{~m} \\
\text { mean: } 1685 \mathrm{~m}\end{array}$ & $\begin{array}{l}\text { min: } 640 \mathrm{~m} \\
\text { max: } 1580 \mathrm{~m} \\
\text { mean: } 998 \mathrm{~m}\end{array}$ & $\begin{array}{l}\text { min: } 935 \mathrm{~m} \\
\text { max: } 1080 \mathrm{~m} \\
\text { mean: } 1000 \mathrm{~m}\end{array}$ \\
\hline $\begin{array}{l}\text { elevation relief } \\
\text { ratio }(\mathrm{E})\end{array}$ & 0.46 & 0.46 & 0.38 & 0.44 \\
\hline slope & $\begin{array}{l}\min : 0^{0} \\
\text { max: } 40.8^{0} \\
\text { mean: } 22.3^{0}\end{array}$ & $\begin{array}{l}\min : 0^{\circ} \\
\text { max: } 40.7^{0} \\
\text { mean: } 22.23^{0}\end{array}$ & $\begin{array}{l}\min : 0^{\circ} \\
\max : 28.5^{0} \\
\text { mean: } 9.5^{0}\end{array}$ & $\begin{array}{l}\min : 0^{0} \\
\text { max: } 4^{0} \\
\text { mean: } 1.08^{0}\end{array}$ \\
\hline $\begin{array}{l}\text { maximum } \\
\text { curvature }\end{array}$ & $\begin{array}{l}\min :-3.0 / \mathrm{m}, \\
\max : 5.9 / \mathrm{m}\end{array}$ & $\begin{array}{l}\min :-3.2 / \mathrm{m} \\
\max : 5.4 / \mathrm{m}\end{array}$ & $\begin{array}{l}\min :-1.2 / \mathrm{m} \\
\max : 3.4 / \mathrm{m}\end{array}$ & $\begin{array}{l}\min :-0.2 / \mathrm{m}, \\
\max : 0.33 / \mathrm{m}\end{array}$ \\
\hline
\end{tabular}




\section{CHAPTER 5}

\section{DATA PREPERATION}

In this section, methods for producing data required as input for the landform classification described.

\subsection{DEM Generation}

First step in data preparation is generating proper DEMs to be used as input for deriving DTMs that constitute the input dataset to be used. There are plenty of sources of DEM as mentioned in section 2.3.1 (DEM). Contours from 1/25000 topographic maps are selected as source elevation data for producing DEMs for the study. This dataset considered proper for representing landscape scale for the study, is widely available, and has standards that are compatible with other topographic contour maps from other countries. Digital contours of 1/25000 scale topographic maps with $10 \mathrm{~m}$ vertical space is obtained from HGK (General Command of Mapping, Turkey). This data also includes several point elevations. Contour lines together with several points that represent specific features like hill tops were utilized for DEM production.

There are two fundamental parameters of DEM generation out of elevation points or contours; method of interpolation and resolution. DEMs for the study are generated using ANUDEM (Austrian National University Digital Elevation Model) procedure (Hutchinson, 1989). This algorithm is particularly developed for contour data and it creates hydrologically correct DEMs (Reuter et al., 2006) which is especially important for deriving DTMs of terrain network, namely; streams and ridges. Grid size depicts the level of abstraction and is related with the scale. Fine resolution DEMs provide detailed representations of terrain where coarse DEMs provide less detail and more generalization. Selection of the DEM resolution constitutes a part of the DEM production progress. Actually, DEMs for the study are not directly used; rather they are used as basis for DTMs 
calculation and scale is mainly managed by means of DTMs. Therefore DEMs are produced at a resolution that portrays the level of detail in the source of elevation, i.e. matching the spatial resolution of the interpolated DEM to the information content of the source contour data.

A series of DEMs were generated with grid sizes of $10 \times 10,15 \times 15,20 \times 20,25 \times 25,30 \times 30$ meters. DEM values are set as floating point to better represent terrain. Samples from shaded reliefs for 'case1' and 'case2' can be seen at Figure 5.1 and Figure 5.2 respectively. Finer resolution DEM i.e. 10x10m DEM depict high local variation where the contour lines pass, so-called contour effect. Coarser resolutions generalize detail that inherently exists in the source dataset. Even though there are methods e.g. standard approach recommended by Hutchinson (1996) to monitor root mean square slope of the DEM as a function of DEM resolution, this method did not point out a significant resolution for DEMs for this study. RMS slope function written in TNTmips macro language SML is given in Appendix A. Error check procedure mentioned below also shows that match between measured values and corresponding cell values are highest for DEM with resolution $15 \times 15 \mathrm{~m} .15 \mathrm{~m}$ DEM is also in the range to map at landscape/mesorelief scale (Dikau, 1989; MacMillan et al., 2004). Therefore DEM resolution for the study is determined as $15 \times 15$ meters.

\section{Evaluation of DEM error}

Evaluation of error in its standard form is comparison of measured values so-called ground truth with the samples from data produced. If the total difference in between is small, than the result is admitted to be proper for particular use. RMS error is very common procedure for evaluation of error; RMS error of a product is compared with RMS error of others produced using different methods or compared to a threshold criteria that represent maximum degree of inaccuracy allowed. RMSE is the root of the square of the errors and defined as:

RMSR $\sqrt{\frac{\sum r_{i}^{2}}{n}}$

Where;

ri: residual of each point

$\mathrm{n}$ : number of test points 

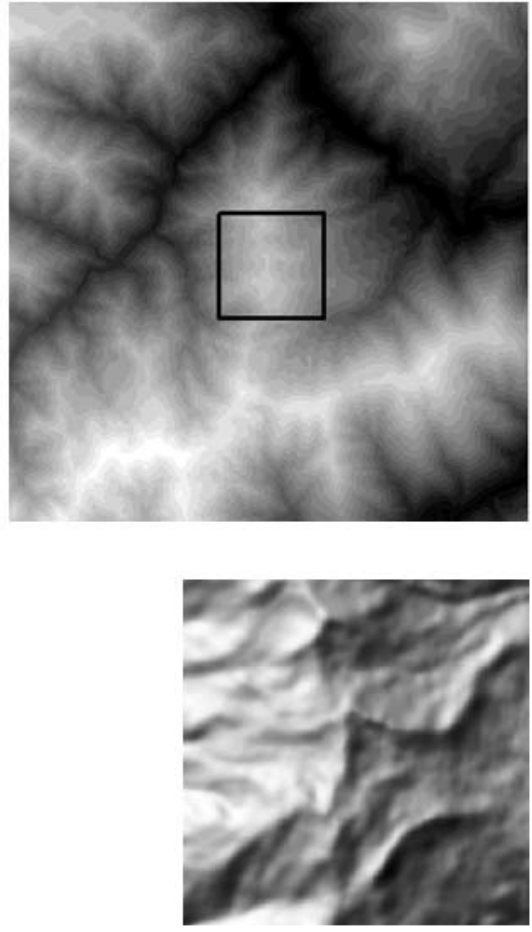

$20 \times 20 \mathrm{~m}$

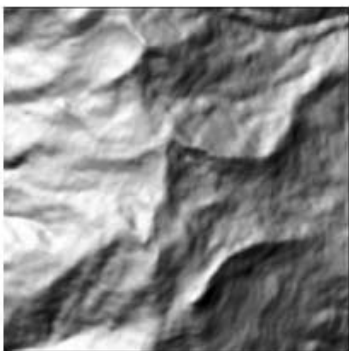

$10 \times 10 \mathrm{~m}$

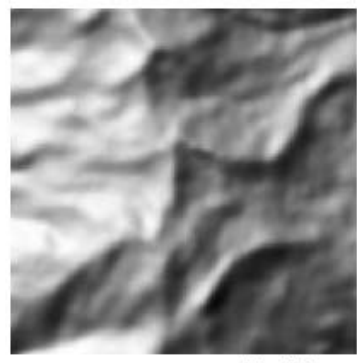

$25 \times 25 m$

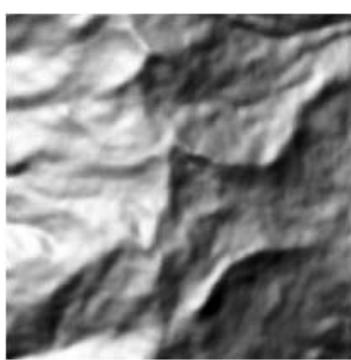

$15 \times 15 \mathrm{~m}$

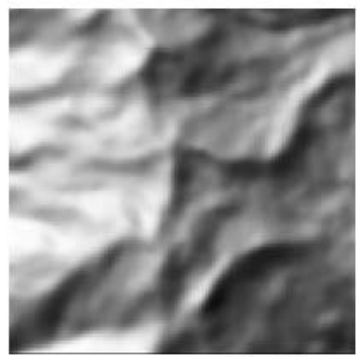

$30 \times 30 \mathrm{~m}$

Figure 5.1: DEM for case1 (bigger scene on the left) and 3 by $3 \mathrm{~km}$ samples of shaded relief for $10 \times 10$ to $30 \times 30$ m resolution DEMs
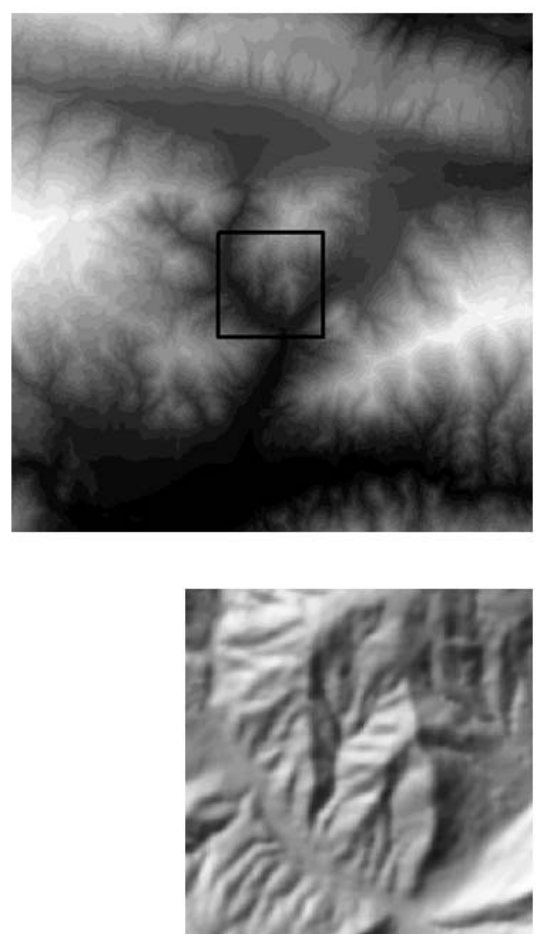

$20 \times 20 \mathrm{~m}$
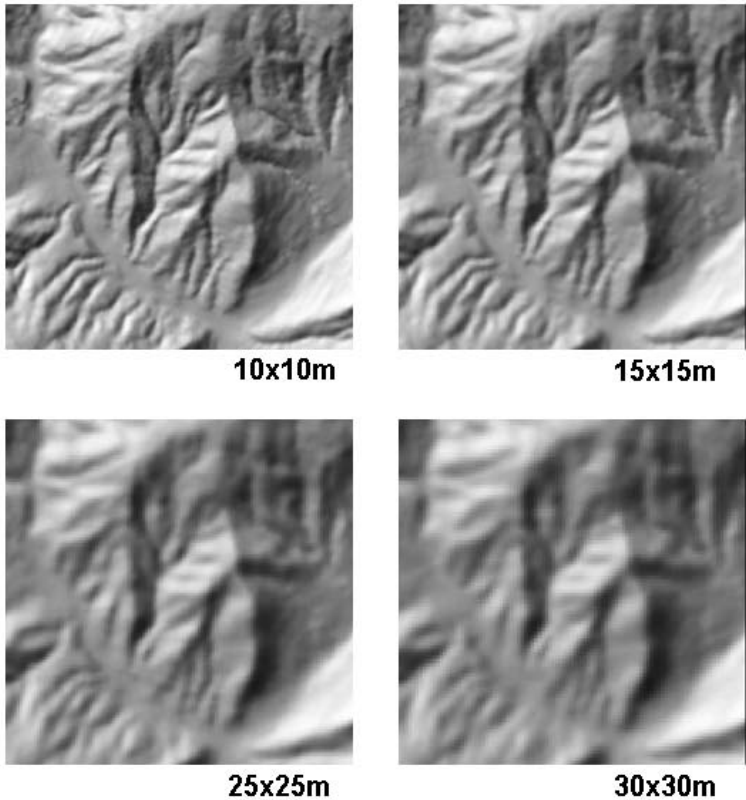

Figure 5.2: DEM for case2 (bigger scene on the left) and 3 by $3 \mathrm{~km}$ samples of shaded relief for $10 \times 10$ to $30 \times 30$ m resolution DEMs 
For error evaluation one needs a set of accurate measurements that are randomly distributed across the area of interest. However these data are usually not readily available and it is not always very easy to collect them on the ground. Measurements with hand GPS also introduce sort of error into ground truthing. Fortunately, source elevation data involved some amount of point measurements in addition to contour lines. About half of those points fall into specific locations such as hill tops which were used as input when interpolating DEMS, remaining points were utilised for error check. A total of 10 points for 'case1' and 8 points for 'case2' were evaluated with corresponding cell values of DEMs with resolutions of $10 \times 10$ to $30 \times 30$ meters, although it is suggested to use more ground control points for DEM accuracy check (USGS, 2009). RMS error for both case1 and case 2 has shown to be lowest for $15 \times 15$ meter DEM (Table 5.1) (see Appendix B for error list of a point elevations versus DEM grids)

Table 5.1: DEM RMS error for 'case1'(top) and 'case2'(bottom)

\begin{tabular}{|r|r|r|r|r|r|}
\hline Case1 & DEM10x10 & DEM15x15 & DEM20x20 & DEM25x25 & DEM30x30 \\
\hline RMS error & 6.307692 & 5.307692 & 18.538462 & 19.076923 & 30.538462 \\
\hline
\end{tabular}

\begin{tabular}{|r|r|r|r|r|r|}
\hline Case2 & DEM10x10 & DEM15x15 & DEM20x20 & DEM25x25 & DEM30x30 \\
\hline RMS error & 2.6020385 & 0.4360853 & 1.007115 & 1.311431 & 0.897977 \\
\hline
\end{tabular}

DEM of $15 \mathrm{~m}$ resolution is small enough to represent inherent scale in the data set given the contour intervals and large enough to escape from the contour effect that is specific to small cell size when interpolating given line or point feature into a continuous surface. Moreover it is more efficient to keep the DEM resolution fine as possible where any generalization can be applied further especially when deriving DTMs.

\subsection{DTMs Derivation}

\subsubsection{Morphometry /Local Geometry}

In this section derivation of two fundamental parameters describing form; 'slope' and 'curvature' from DEM (first and second derivatives) at particular scales is described. Slope is the rate of change of elevation in both $\mathrm{x}$ and $\mathrm{y}$ directions and can be used to identify the direction and magnitude of steepest gradient (Jones, 1998). Terrain curvature for a particular cell represents the curvature of a line formed by intersecting a plane of some chosen orientation with the terrain surface. 
In this study full set of five morphometric DTMs is used; slope, plan, profile, minimum and maximum curvature similarly as described in Dikau's scheme (1989).

Quadratic approximations are known to produce more accurate terrain derivatives compared to other common methods (Florinsky 1998). For this study, a local quadratic approximation of surface as specified by Evans (1972) and Young (1978) and customized by Wood (1996) for larger kernels is employed. Algorithm is sensitive to size of the local window that is used to calculate the derivatives. A single compromised window size that portrays characteristics of terrain at a specific scale that is landscape scale is proposed for this study. Specific window size successfully represent features at the particular scale defined by the window, however poorly represents features that are reasonably small or larger than that window size. Therefore, windows size should regard the scale of the intended work and be capable of capturing the landscape character. Some authors associated terrain parameters with some specific phenomena like soil (Schmidt and Hewitt, 2004) and chose the window size providing best correspondence as the optimum window size, but this is rather a confirmation that the window size is set to approximate the scale of the specific phenomena. For this study window should; (i) represent scale of the interest, which is predefined as mesorelief A (Dikau, 1989) (ii) cover majority of features dominating the terrain at the scale range of interest.. This range is coarser than hill slope scale or 'microrelief' hence can be associated with 'Mesorelief A' according to the hierarchical levels of landform units as described by Dikau (1989). Mesorelief A covers landform elements that broadly range between $10^{4}$ to $10^{6} \mathrm{~m}^{2}$ in areal extents. Initial morphometric classifications shown that average size of elements that approximate mean areas of $10^{5}$ could be gathered using window sizes of about $500 \times 500 \mathrm{~m}$ to $1000 \times 1000 \mathrm{~m}$.

Scale in this study is managed through window sizes that are used to calculate DTMs. Accordingly, quadratic approximation of surface through a range of successively increasing window sizes given; $15 \times 15,25 \times 25,35 \times 35,45 \times 45,55 \times 55,65 \times 65$, and $75 \times 75$ is employed to calculate slope and curvature parameters. A series of maximum curvature for 'case1' at varying scales which portrays important features of terrain is illustrated in Figure 5.3.

Search for characteristic scale on the other side is implemented by employing Wood's characteristic scale approach and with another self suggested method built on a set of assumptions. 

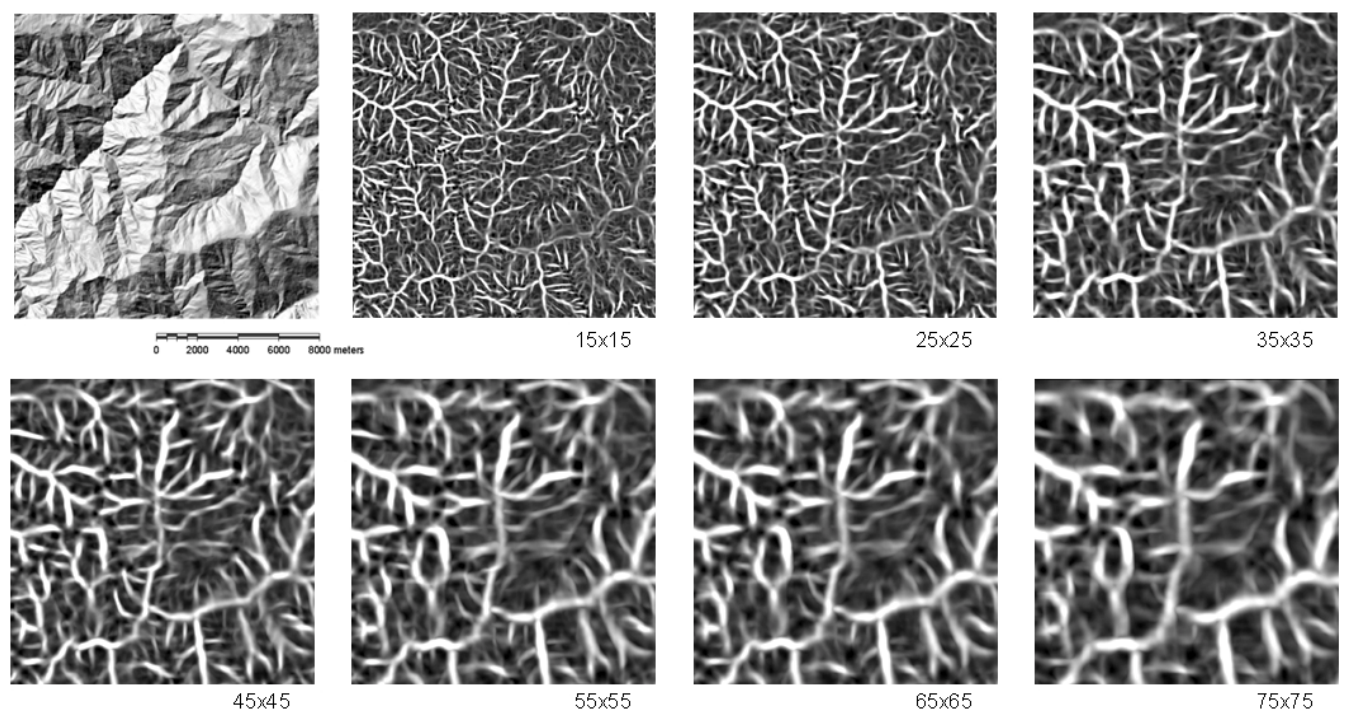

Figure 5.3: Shaded relief of case1, maximum curvature over a range of windows sizes; $15 \times 15,25 \times 25,35 \times 35,45 \times 45,55 \times 55,65 \times 65$, and $75 \times 75$ that is $75 \times 75 \mathrm{~m}, 225 \times 225 \mathrm{~m}, 375 \times 375 \mathrm{~m}$, $525 \times 525 \mathrm{~m}, 675 \times 675 \mathrm{~m}, 825 \times 825 \mathrm{~m}, 1125 \times 1125 \mathrm{~m}$ respectively for 'case 1 '.

Wood's method of searching a characteristic scale involves calculation of DTMs i.e. curvatures using a successively increasing window sizes, for each grid, window size where curvature reaches its maximum value is recorded. Frequency histograms of those window sizes are plotted. Those window sizes are considered to reveal characteristic scales at which a particular surface parameter appears most extreme. DTMs are calculated by and evaluated for characteristic scale with Landserf script 'Charscale' (Appendix C). Evaluations started with small scale ranges i.e. $3 \times 3$ window to $25 \times 25$ window, it was observed that the largest window size yields the maximum value, it is enlarged up to $300 \times 300$ window size, which also takes a lot of computation time, because there are usually some features like mountains that remain larger than the window size, so they produce high curvature values even the window is very large that it can cover the whole scene. This problem is also mentioned by Barringer et al. (2009) as; 'Attempts to determine a dominant landscape scale have so far tended to recognize the larger features as dominant, and resulted in oversimplification of the landform elements on hillslopes (spurs, hollows, etc.)'.

Some of the evaluation results for which maximum window size of $25 \times 25$ is used (Figure 5.4), maximum window size of $45 \times 45$ is used (Figure 5.5) and maximum window size of $85 \times 85$ is used (Figure 5.6) shows it is likely that there is a clustering around scale determined by $15 \times 15$ window. However it is still a question how to eliminate largest window size, as it is consuming some amount of area and obscuring results. 


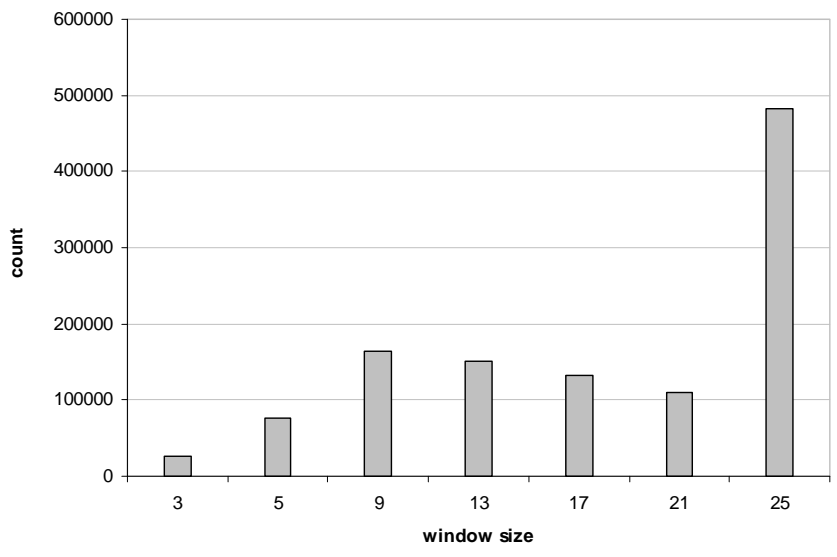

Figure 5.4: Characteristic scale results for window sizes from $3 \times 3$ to $25 \times 25$.

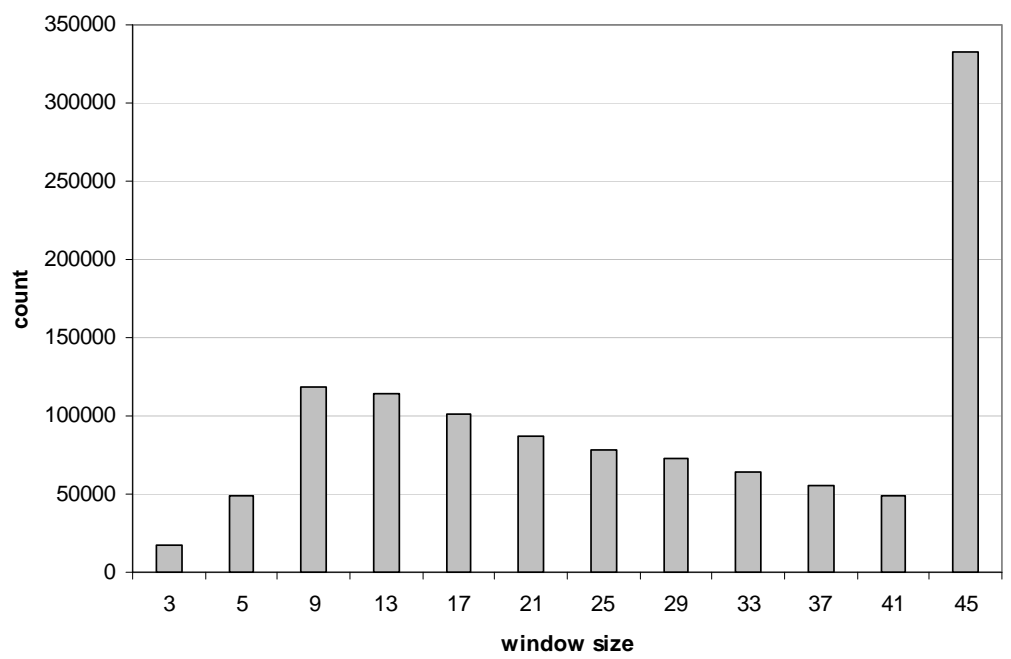

Figure 5.5: Characteristic scale results for window sizes from $3 \times 3$ to $45 \times 45$.

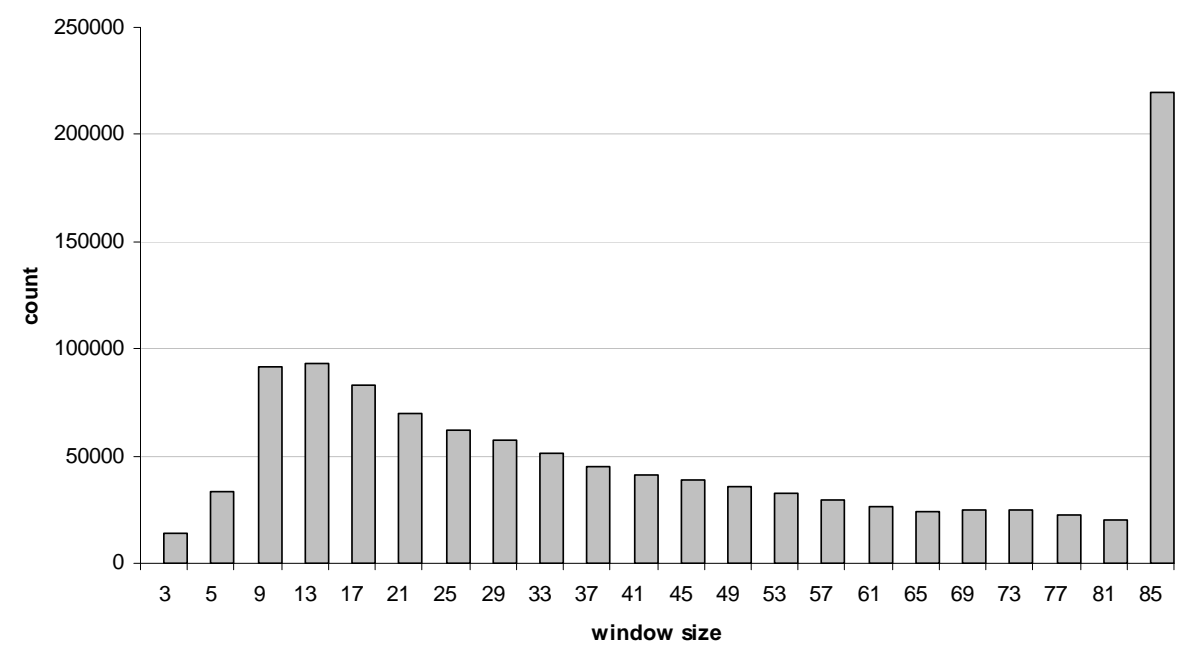

Figure 5.6: Characteristic scale results for window sizes from $3 \times 3$ to $85 \times 85$. 
The other characteristic scale evaluation rely on the assumption that high curvature value indicates that a given window size effectively captures the full curvedness of a feature, a smaller or a larger size of window would yield lower curvature values (Figure 5.6)

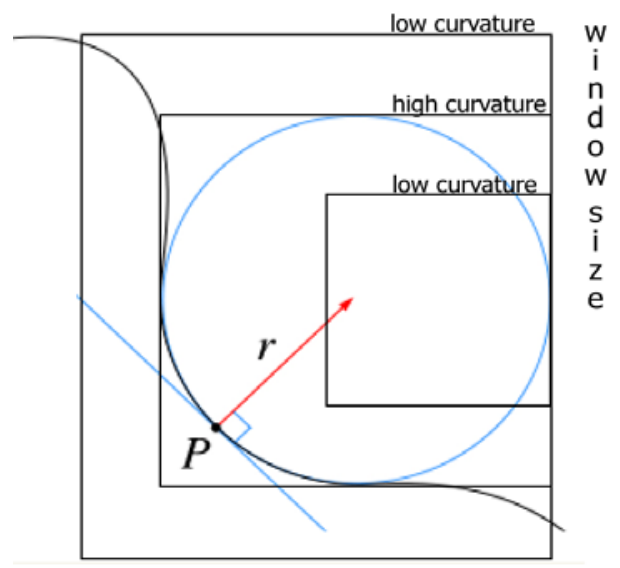

Figure 5.7: Varying window sizes imposed over a curved feature.

If the overall values of a curvature parameter calculated with a given window size is relatively high compared to others, then this window size is likely to capture majority of features that pertain to the scale inherent in the given window size.

Maximum curvature among the other morphometric DTMs is considered to represent landform morphometry more closely. Therefore, maximum curvature is evaluated for characteristic scale, but before concave part is removed from the computations as curvature values both negative and positive tend to shift the mean value towards zero which may yield improper results. Characteristic scale evaluation is made on convexities of maximum curvature. Table 5.2 includes global mean of convexity calculated for a series of window sizes, and Figure 5.7 illustrates the plot of the mean values.

Table 5.2: Summary statistics; mean and standard deviation for maximum curvature convexity calculated for a range of window sizes.

\begin{tabular}{|c|r|r|}
\cline { 2 - 3 } \multicolumn{1}{c|}{ Window size } & mean & Standard deviation \\
\hline $15 \times 15$ & 1.077263 & 0.985139 \\
\hline 25 & 1.26405 & 1.099788 \\
\hline 35 & 1.218457 & 1.017353 \\
\hline 45 & 1.314952 & 1.077629 \\
\hline 55 & 1.20649 & 0.97001 \\
\hline 65 & 1.296651 & 1.03862 \\
\hline 75 & 1.182856 & 0.933335 \\
\hline
\end{tabular}




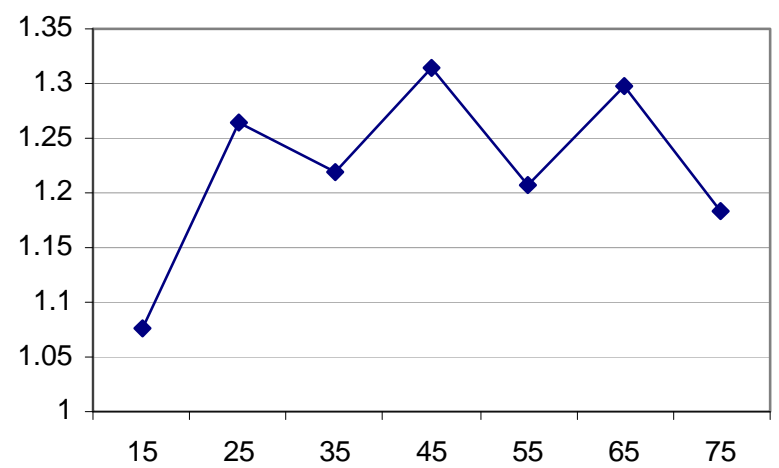

Figure 5.8: Graph for mean for maximum curvature convexity calculated for a range of window sizes.

Window size $45 \times 45$ which is $675 \times 675 \mathrm{~m}$ on the ground is likely to capture a characteristic scale, and classes of average size around $30000 \mathrm{~m}^{2}$ can be produced which is in the range of Mesorelief A, therefore this window size can be accepted as the core window size of the analysis scale. $15 \times 15$ also depicted a characteristic scale according to Wood's (2009) characteristic scale analysis, therefore it is taken as the finer analysis scale, $75 \times 75$ is taken as the coarse scale. Therefore a range in between $15 \times 15$ to $75 \times 75$ kernel for DEM of $15 \mathrm{~m}$ which corresponds to $225 \times 225 \mathrm{~m}$ and $1125 \times 1125 \mathrm{~m}$ is taken as fine and coarse scale limits for the study. Mid scale is represented by $45 \times 45$ matrix, $675 \times 675 \mathrm{~m}$ is considered to well represent Mesorelief A. Although it is better to give window size in metric units to reveal real measurements, in this study window size in cell units is preferred for simplicity, one should understand that $45 \times 45$ window points out $675 \times 675 \mathrm{~m}$ on surface, and similarly as below 'case1' is produced in multiple scales and each of the case datasets produced this way is referred to as 'case1 fine', 'case1' and'case1 coarse' (Table 5.3).

Table 5.3: Multiple scales of datasets and their corresponding cases

\begin{tabular}{|c|c|c|c|}
\hline DEM resolution & Window size in cell units & Window size in meters & Case \\
\hline \multirow{3}{*}{$15 \times 15 \mathrm{~m}$} & $15 \times 15$ & $225 \times 225 \mathrm{~m}$ & case 1 fine \\
\cline { 2 - 4 } & $45 \times 45$ & $675 \times 675 \mathrm{~m}$ & case 1 \\
\cline { 2 - 4 } & $75 \times 75$ & $1125 \times 1125 \mathrm{~m}$ & Case1coarse \\
\hline
\end{tabular}

DTMs for the area are calculated for case 1 (Figure 5.9) at three scales $15 \times 5$ (Figure 5.10), $45 \times 45$ (Figure 5.11), $75 \times 75$ (Figure 5.12). That is to evaluate effect of scale on landform classification and to test if the method still works for different scales. DTMs for 'case2' (Figure 5.13) are calculated at $45 \times 45$ scale (Figure 5.14). 
Morphometric DTMs illustrated for sample areas that lay in the mid of the cases with areas of $3 \times 3 \mathrm{~km}$ for better visualization. DTMs for 'case1next' are calculated at $45 \times 45$ scale but they are not are not illustrated as their characteristics are quite the same as case1.

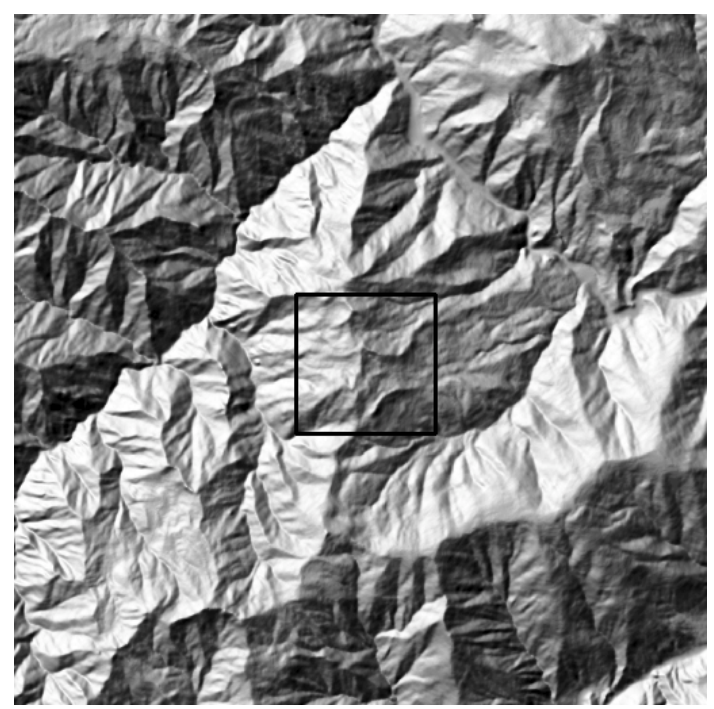

Figure 5.9: Location of $3 \times 3 \mathrm{~km}$ sample area in the mid part of the study area case 1 .
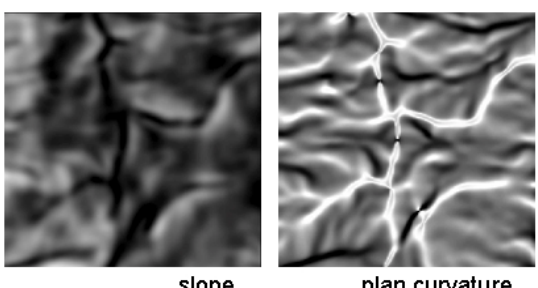

plan curvature

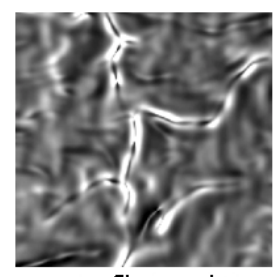

profile curvature

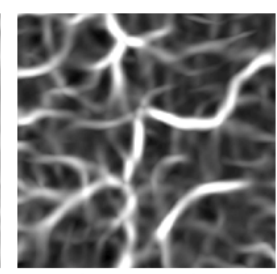

maximum curvature

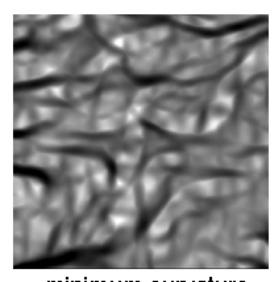

minimum curvature

Figure 5.10: Morphometric DTMs of $3 \times 3 \mathrm{~km}$ sample area for case 1 calculated at scale $15 \times 15$.

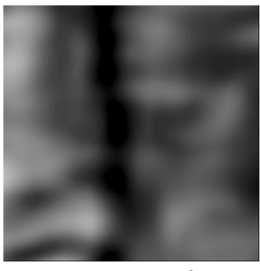

slope

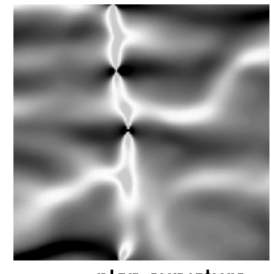

plan curvature

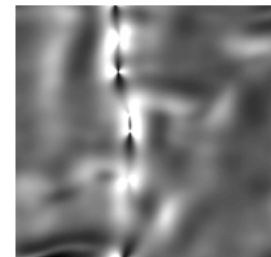

profile curvature

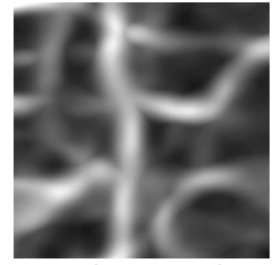

maximum curvature

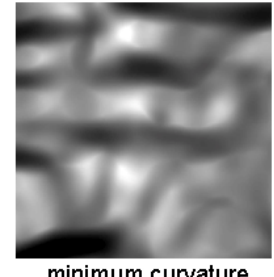

minimum curvature

Figure 5.11: Morphometric DTMs of $3 \times 3 \mathrm{~km}$ sample area for case 1 calculated at scale $45 \times 45$.
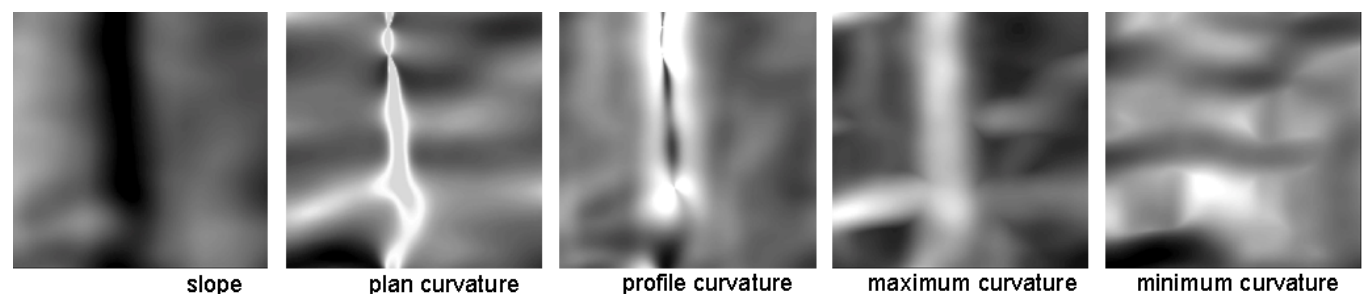

Figure 5.12: Morphometric DTMs of $3 \times 3 \mathrm{~km}$ sample area for case 1 calculated at scale $75 \times 75$. 


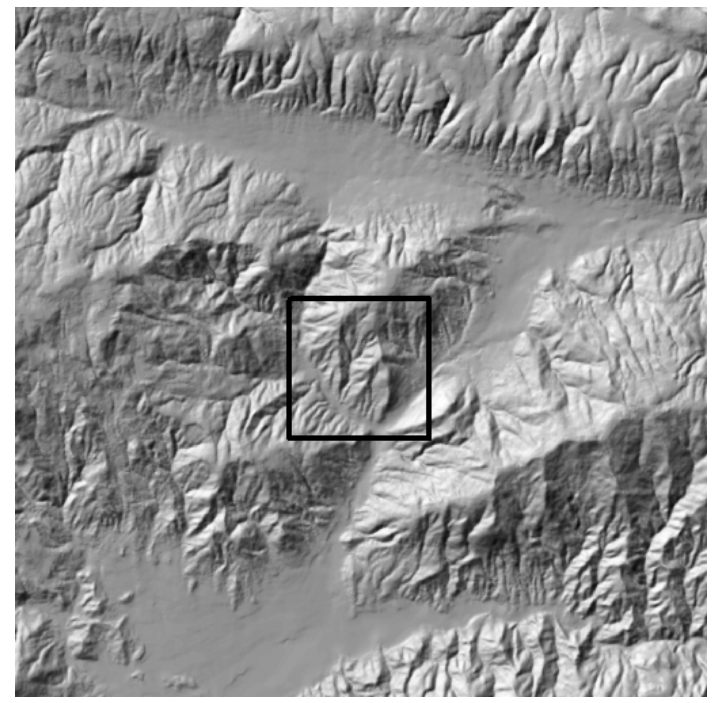

Figure 5.13: $3 \times 3 \mathrm{~km}$ sample area in the mid part of the study area case 2 .
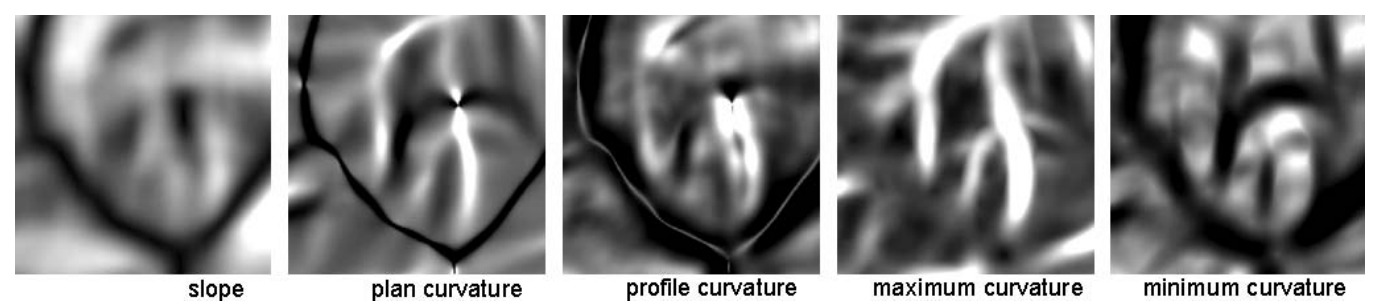

Figure 5.14: Morphometric DTMs of $3 \times 3 \mathrm{~km}$ sample area for case 3 calculated at scale $45 \times 45$.

\subsubsection{Geomorphometric context}

\subsubsection{Terrain Position}

Among the terrain position indices mentioned in Section 2.3.2.2.1 (Terrain Position); methods based on 'surface flow' had inefficiencies in depicting high differences of flow values especially between streams and adjacent pixels, 'proximity to terrain networks' methods yield unexpected values at complex terrain. Therefore Topographic Position Index (TPI) which is a 'local elevation' based method is employed. TPI simply compares the elevation of each cell in a DEM to the mean elevation of a specified neighborhood around that cell. Therefore topographic position calculated this way is scale-dependent. It is important to set window size to properly represent the relative position across all the given area. Fels and Matson (1996) suggest that radius of search distance should be one-half of the 
fractal dimension of the landscape, that is, one half of the ridge-to-stream distance in that landscape. A proper window size is obtained based on this assumption.

Ridge-to-stream distances where some of which are shown in Figure 5.15 are measured both for 'case1' and'case2'. Only major ridges and streams are considered. Accordingly ridge-to stream distance for case 1 can be said to be clustering around approximately $3000 \mathrm{~m}$, and for case 2 distance is larger around $3500 \mathrm{~m}$. One-half of these distances which equals to 1500 and 1750 for case 1 and case 2 respectively were employed as search distance radius for TPI calculation suggested by Jenness Enterprices (Jenness, 2006).
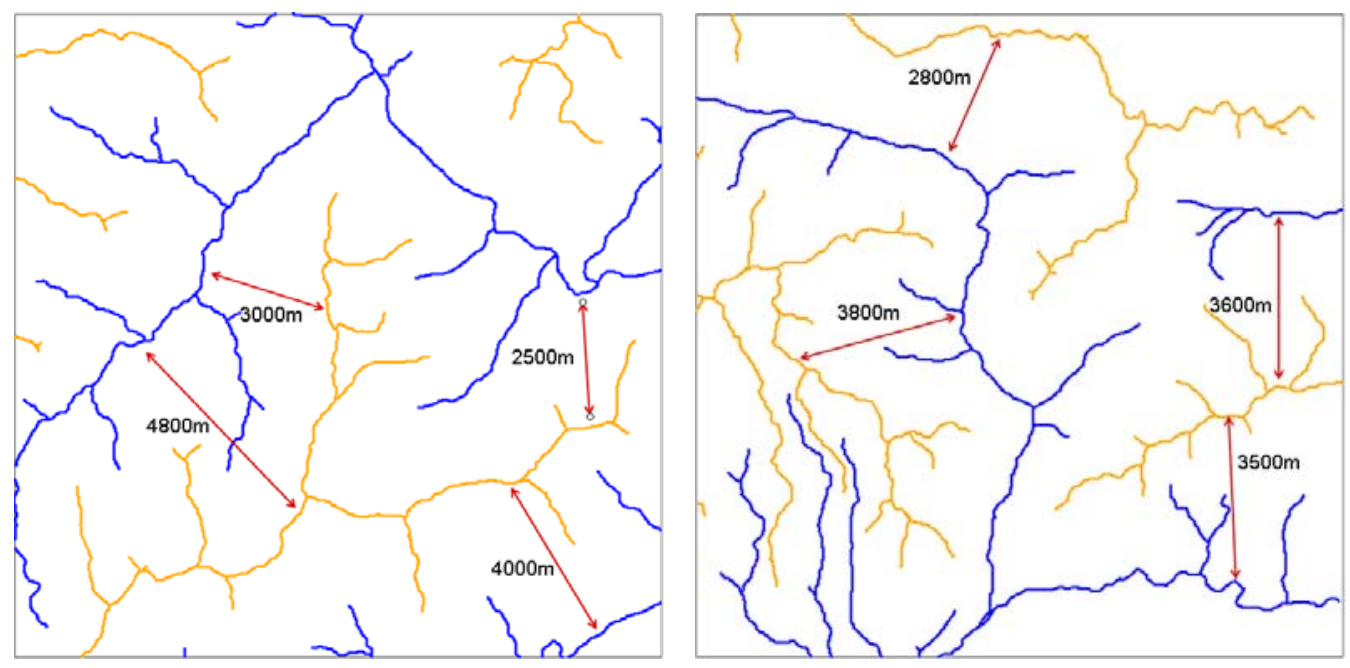

Figure 5.15: Ridge to stream distances for case1 (on the left) and case2 on the right. Blue color indicates streams and yellow color indicates streams

Results for TPI for 'case1' and 'case2' are provided in figures 5.16 and 5.17 respectively. Both of the TPIs show similarity to DEMs, however higher altitudes are not necessarily assigned with highest TPI values. That is what characterizes the relative position; where high altitudes, but not necessarily highest altitudes constitute the upper positions. 

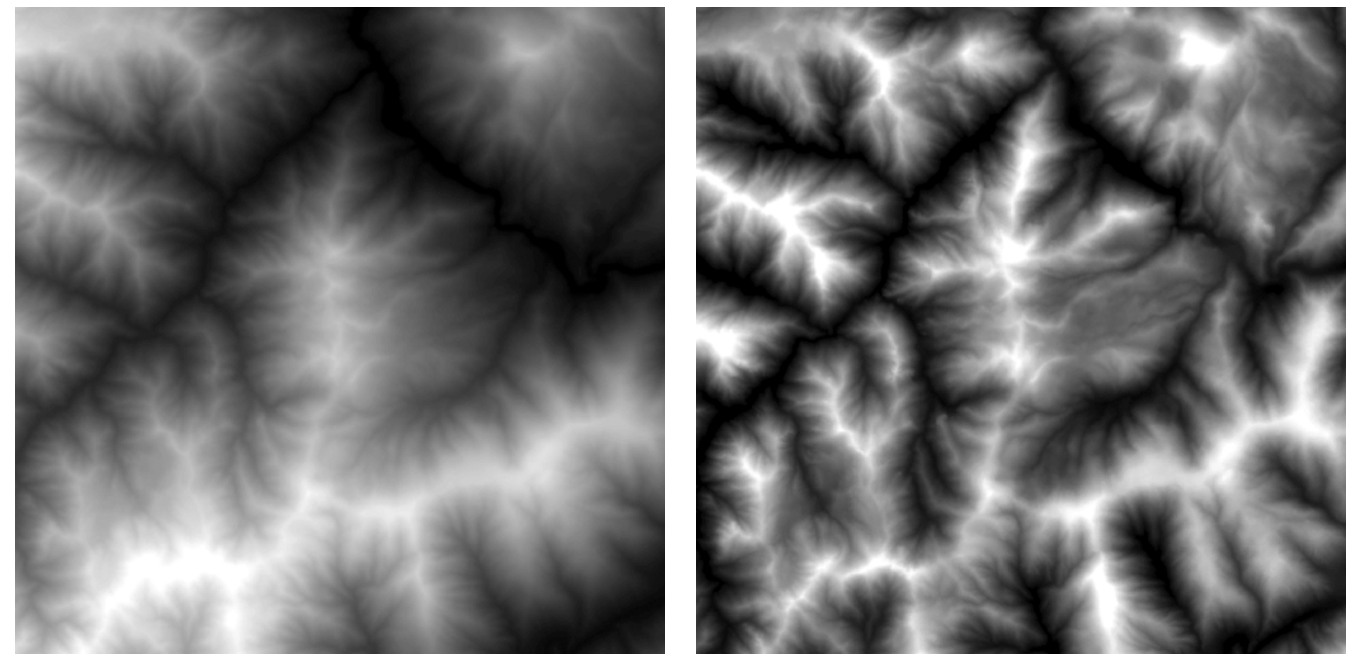

Figure 5.16: DEM for case1 (left) TPI for case1 (right).
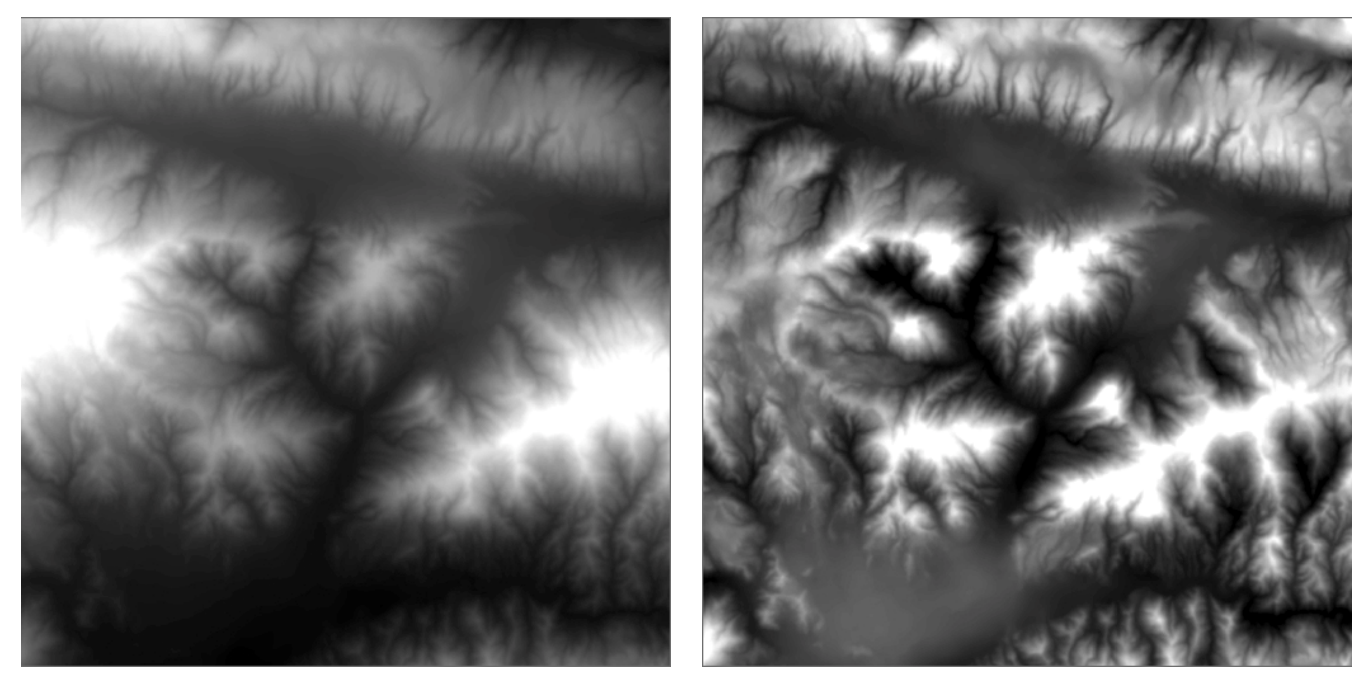

Figure 5.17: DEM for case2 (on the left) TPI for case1 (on the right).

\subsubsection{Terrain Network}

Among the terrain network extraction algorithms given in section 2.2.3.2.2 (Terrain Network), Hydrographic Approach is considered to be proper way of extracting terrain networks, so-called streams and ridges or divides in hydrographic terminology.

Hydrographic approach models the surface flow across the landscape. A flow direction and flow accumulation is produced where each hypothetic rain drop flows to a direction and accumulates into another cell. Where flow accumulation per pixel exceeds a minimum threshold is described as flow path that construct a stream network or drainage pattern 
across the landscape. Flow paths are usually put into hierarchal orders of magnitude (Horton, 1945; Strahler, 1964; Shreve, 1966).

A multi-flow algorithm available in TNTmips was employed to construct streams and ridges. This algorithm is superior in a way that it produces connected streams and streams can be produced at some orders of magnitude. In this study, Strahler order which is most widely used is employed. There is up to 7 Strahler orders of magnitude for case1 (Figure 5.18) where 7 denote the strongest stream.

To extract ridge lines for the study, watersheds and basins are extracted first. Sub-basin boundaries trace ridges at some parts but they can trace valley bottoms as well so as to close the boundaries of sub basins. At this point inverse DEM is utilized to obtain inversed streams which become ridges. Flow accumulation algorithm is applied on an inversed DEM to calculate streams, which become ridges. DEM is inverted using a simple arithmetic operation such;

$$
D E M_{i n v}=\operatorname{abs}(D E M-(D E M \max +D E M \min )
$$
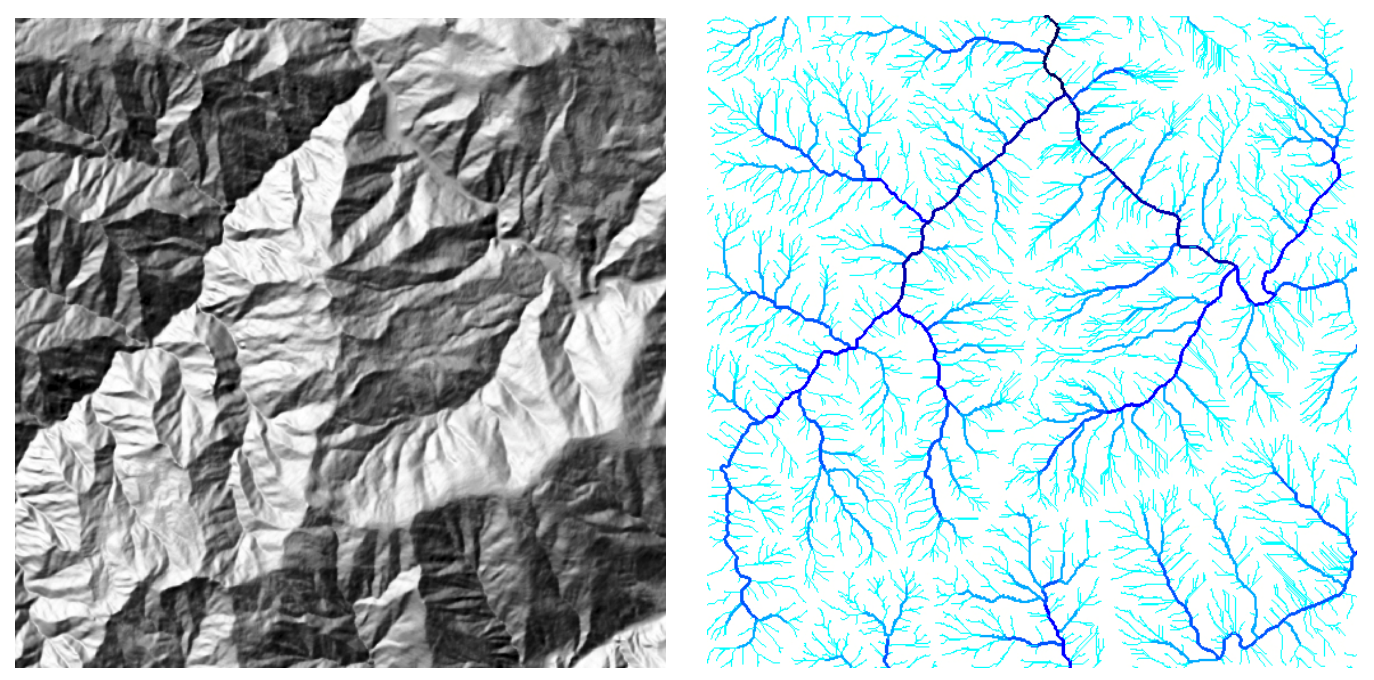

Figure 5.18: Case1 (left), streams of Strahler order (right). Light blue to dark blue represents increase in orders of magnitude. 
So that minimum altitude replaces with maximum altitude and the data in-between is inverted linearly. In the flow accumulation algorithm Sinks are not filled because they become the peaks.

This method also produces ridges in orders of magnitudes similarly as streams so that only higher orders of magnitudes can be selected to represent ridges (Figure 5.19). After inversed streams (ridges) are derived, some inconsistencies in ridges are corrected taking basin boundaries as reference that are more correct compared to ridges derived this way.

Streams and inverse streams (ridges) of orders of magnitude 4 and higher for case 1 are illustrated in Figure 5.20. These two linear features are used as input to represent 'terrain network' for geomorphometric classification of case1.
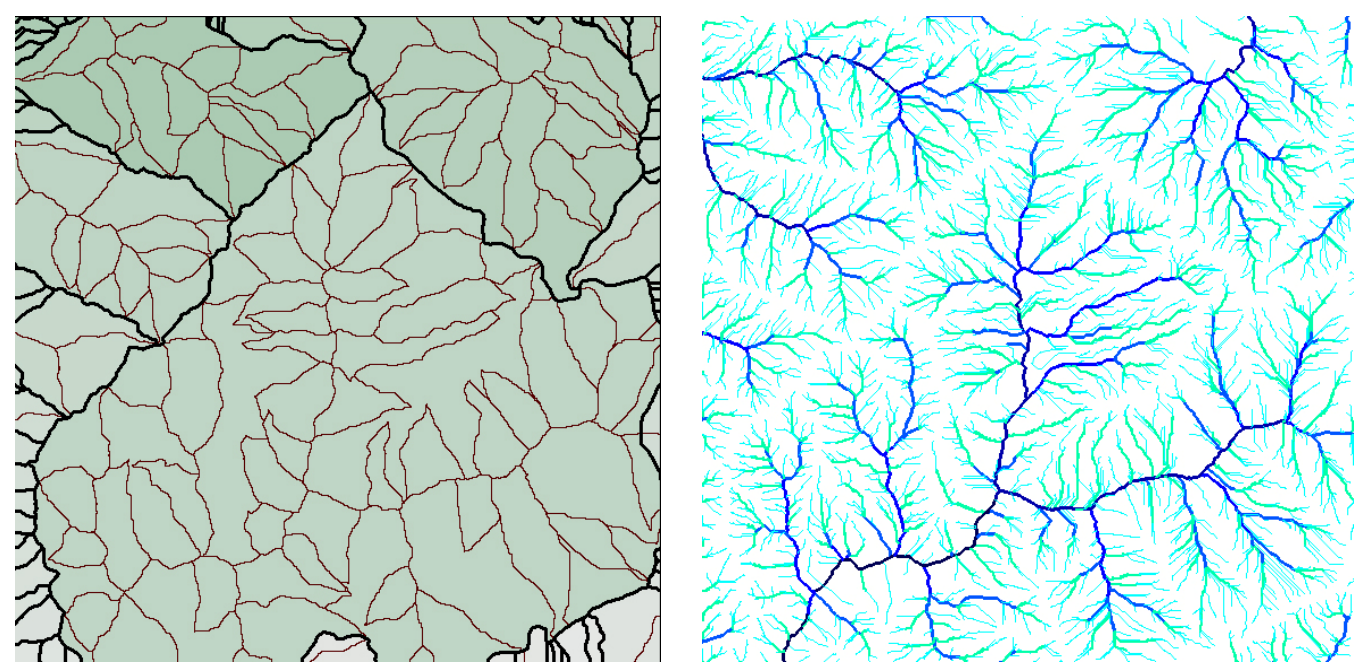

Figure 5.19: Watersheds and basins (on the left), inverse DEM streams (on the right) for case1. 

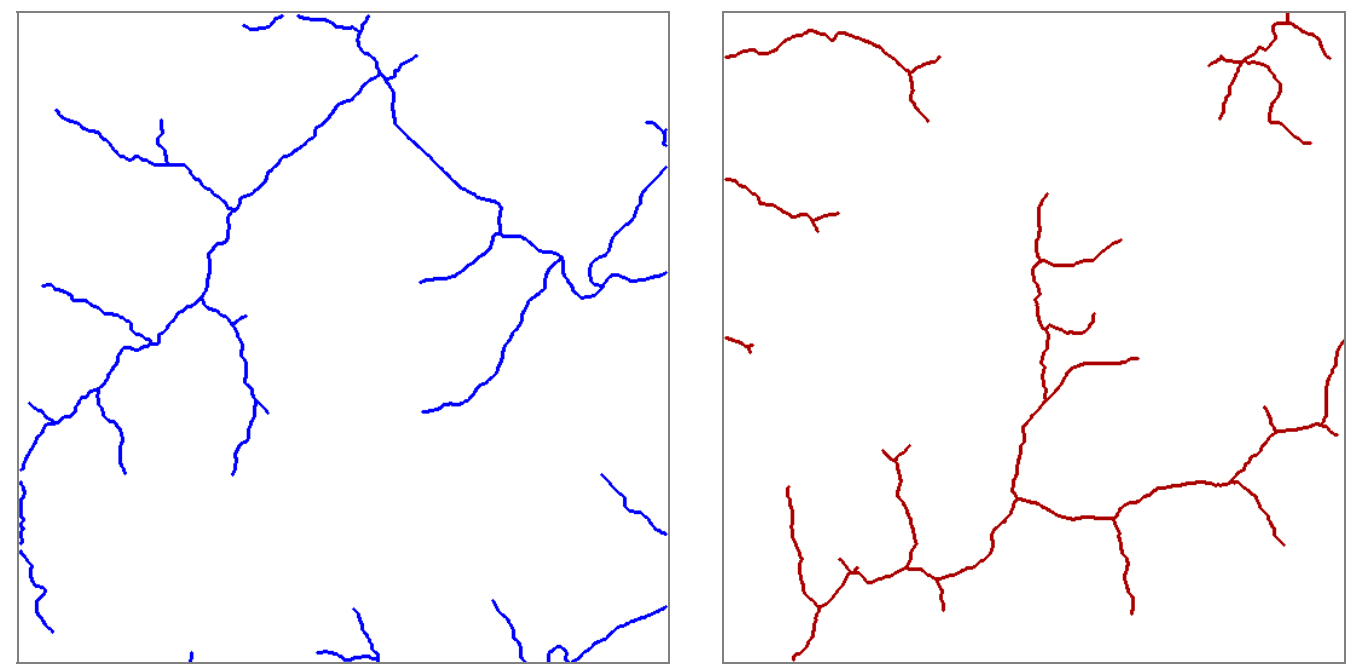

Figure 5.20: Streams (left), ridges (right) used as terrain network features in geomorphometric classification of case1.

Same set of procedures is applied for deriving terrain network features for case2. A flow accumulation raster is generated out of DEM and streams are extracted in Strahler orders of magnitude (Figure 5.21).
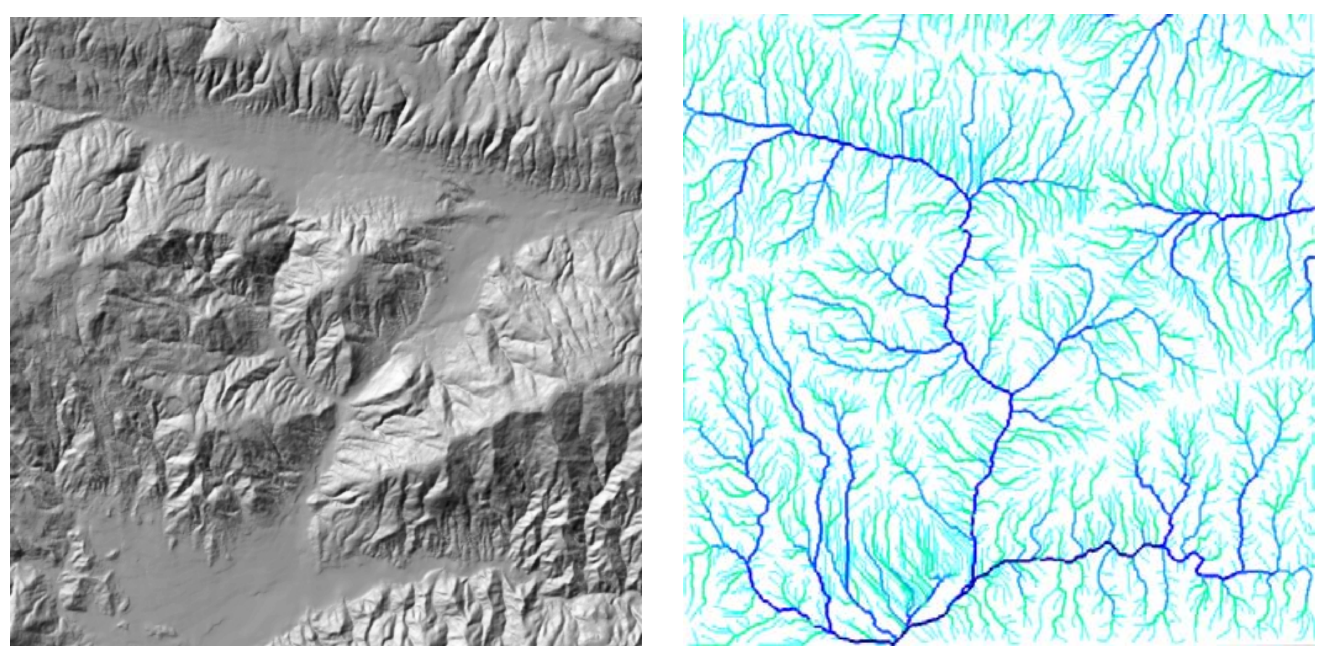

Figure 5.21: Case1 (left), streams of Strahler order (right). Light blue to dark blue represents increase in orders of magnitude.

Watershed and sub-basins are derived, an inverse stream (ridge) is generated out of an inverted DEM of case2 (Figure 5.22). 

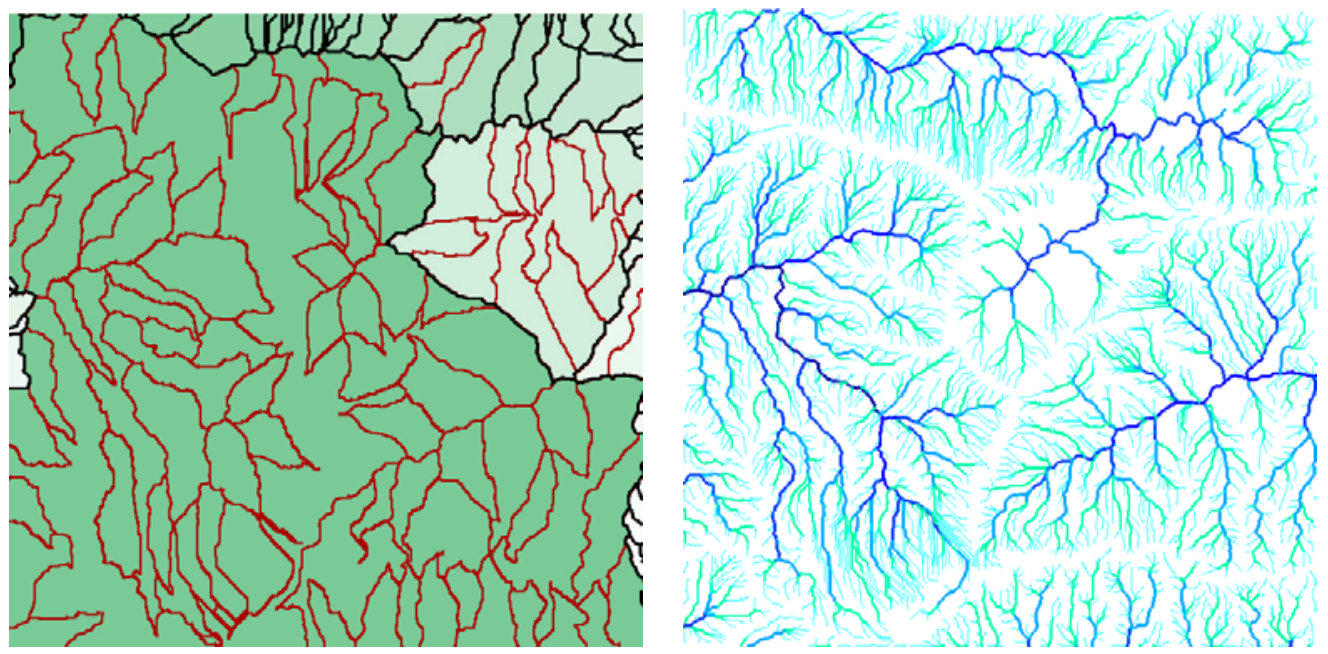

Figure 5.22: Watersheds and basins (on the left), inverse DEM streams (on the right) for case2.

Streams and inverse streams (ridges) for cse 2 are produced features with orders of magnitude 4 and higher are extracted Figure 5.23. These two linear features are utilized as terrain network features for geomorphometric classification of case2.
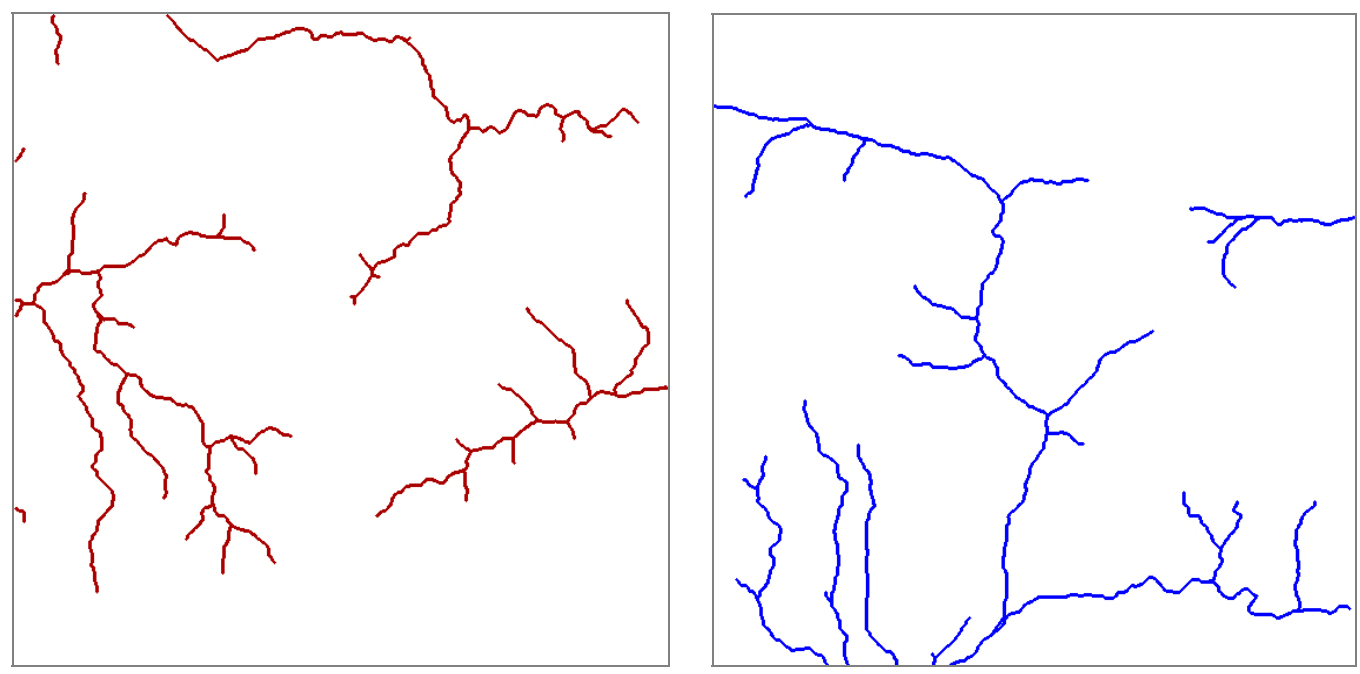

Figure 5.23: Streams (left), ridges (right) used as terrain network features in geomorphometric classification of case2. 


\section{CHAPTER 6}

\section{OBJECT-BASED CLASSIFICATION OF LANDFORMS}

A methodology is presented for the object-based analysis and classification of landforms based on local geometry and geomorphometric context. Segmentation constitutes initial part of the analysis. Homogeneous objects are obtained and utilized as basic entities for the fuzzy classification of morphometric landforms through semantic import model (SI). Morphometric classes are then reclassified using a set of logical rules that incorporate geomorphometric context through multi-level relationships of objects.

\subsection{Segmentation}

Segmentation operation yields homogeneous contiguous objects that form the basis of Object-based image analysis. Segmentation procedure aggregates individual pixels into 'objects' and 'objects' which carry far more information than spectral values become the basic spatial carriers of information for further analysis. This is a shift from pixel domain into objects domain for classification.

A multi-resolution segmentation based on Fractal Net Evaluation Algorithm (FNEA) (Baatz and Schape, 2000) is employed for this study. A segmentation that is utilized to produce three hierarchical levels is employed. Initial segmentation uses morphometric DTMs as input, i.e.; slope, plan, profile, maximum, minimum. Whole scene is segmented into homogeneous entities (objects) of form at the relevant scale. This segmentation level is labelled as 'Level -1 ' as it constitutes the bottom level in the hierarchy. 'Level 1' which carries positional information constitutes the top level. Geomorphometric classification is implemented at the mid level that is 'Level 0' (Figure 6.1). 


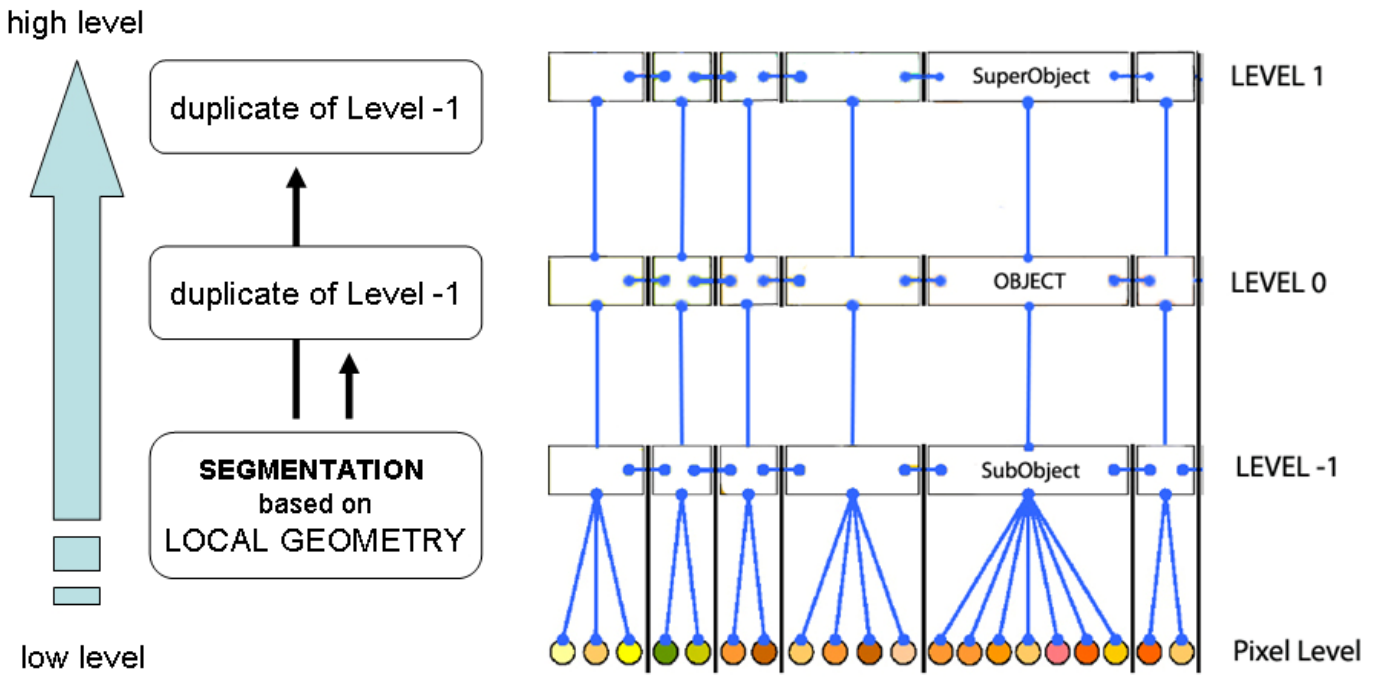

Figure 6.1: Hierarchical multi-level segmentation proposed for geomorphometric classification.

Once a level of objects are created by segmentation, another level can be created at a higher or lower by segmenting the original level again with a lower or a higher scale parameter respectively or another segmentation can be obtained just by simply duplicating the original level into a higher or lower position in the hierarchy. In the proposed study, segmentation based on morphometric DTMs at "Level -1" is duplicated without any modification to 'Level 0' which is a higher level. 'Level 1' is similarly produced by duplication of 'Level 1', over 'Level 0' and located at the top of the hierarchy.

Multi-level segmentation is widely used to represent different scales for a given dataset; hence it involves nested hierarchies of scales (Hall et al., 2004). However, this study adopts particular scales and performs the classification for each scale independently. Segmentation is rather employed to utilize sub-object super object relationships within a hierarchy of levels for classification. Objects of 'Level -1 ' and 'Level 1' according to 'Level 0' are formally called 'sub-objects' and 'super objects' in eCognition environment.

Morphometric classification is implemented at 'Level -1', terrain position classification is implemented at 'Level 1'. Level 0 which is in between incorporates information from both 'Level -1' and 'Level 1'. This sub-level, super-level information is further utilized in geomorphometric classification that is implemented at Level 0. 


\section{Segmentation Parameters:}

A region growing segmentation algorithm 'Fractal Net Evaluation Algorithm (FNEA)' developed by Baatz and Schäpe (2000) that is embedded in eCognition software is employed to obtain objects prior to classification for the study. Accordingly, algorithm merges individual pixels in a pairwise fashion into larger objects until a heterogeneity criteria defined by the user is reached. Segmentation procedure requires particular parameters to be defined before the operation takes place. These are: (i) scale parameter (ii) weight of the spectral attribute versus shape (iii) within shape criteria; weight of compactness versus smoothness, (iv) weight of the input layers used in segmentation. First three of these criteria are schematically represented in Figure 6.2.

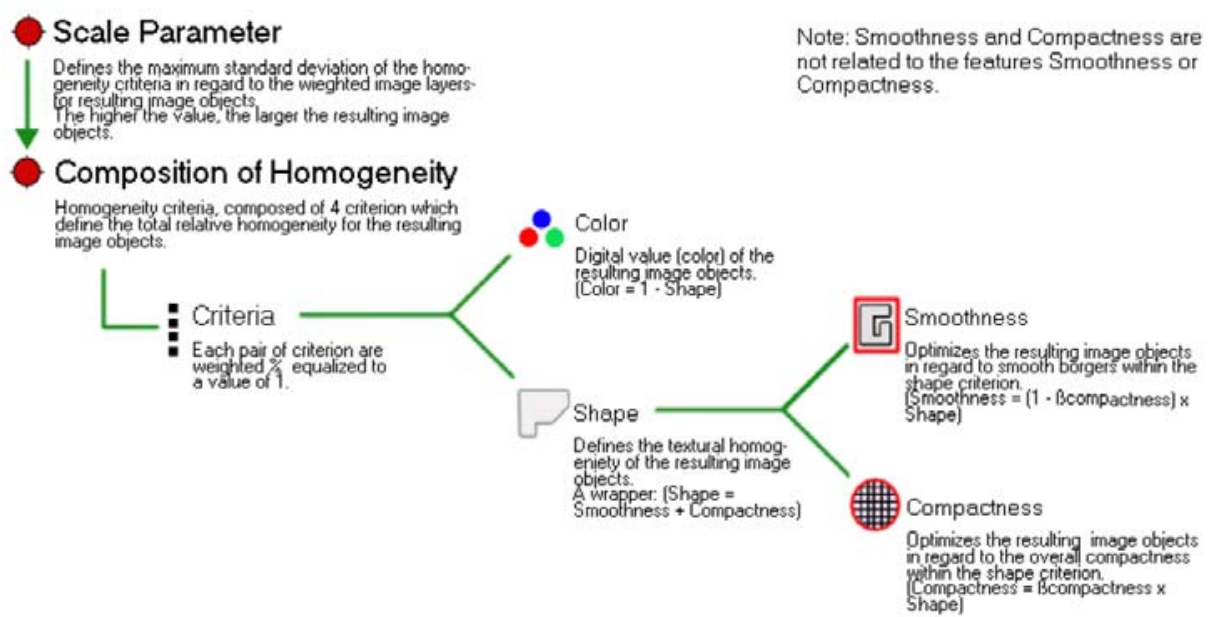

Figure 6.2: Schematic representation of segmentation parameters (eCognition Developer v.8, Reference Book).

To start with the input layers, and leave the scale which is the most crucial part to the end; slope, plan curvature profile curvature, maximum curvature and minimum curvature are given as input into segmentation process at 'Level -1'. Aim is to obtain objects of homogeneous form characteristics. Even though the data ranges of layers are different, FNEA handles them at equal weights, given the equal weight parameters. For the study all input layers are weighted the same. 'Color' represents attribute space and 'Shape' represents geometry of the object. 'Color' and 'Shape' criterion are 0.9 , and 0.1 respectively by default. Their values should both sum up to 1 , e.g. if shape criterion is increased to 0.2 , 'color' criterion automatically decreases to 0.8 . After a series of evaluations it was observed that increasing weight of the shape yield misrepresentative segments of terrain form. That is considered to be related with the continuous nature of terrain which do not pose some 
regular 2D shapes as could be observed in the satellite imagery. Therefore shape criterion is left 0.1 which automatically give weight of 0.9 to attributes coming from morphometric DTMs.

Scale parameter is a crucial threshold that determines the maximum allowed heterogeneity for segmentation, which has a direct influence on the size of the objects to be obtained. Scale parameter after a trial and error process is recognized to be within particular ranges for the case areas and sets of DTMs of different scales. This scale range is approximately between $5-12$. A scale threshold that is smaller than ' 5 ' produced very fine objects where segmentation over-subdivides the scene and creates small groups of pixels. Utilizing an oversegmented layer with very fine objects is not an efficient way of employing full effectiveness of segmentation. Over segmented scene is little far from the pixel domain as the objects are composed of few pixels. This layer might still suffer from the weaknesses of pixel domain like scattered classes all over the scene. A threshold that is greater than '12'on the other side produced coarse objects where some of the important inherent detail gets lost. An optimal scale within the range was needed however it is also important to obtain a quantified measure of scale rather than just visually compromised one. Most of the authors suggest a series of trial and error procedure and some others suggest some optimization tools for an optimal segmentation. A novel method that is introduced by Dragut et al. (2010) 'Estimation of Scale Parameter (ESP) that builds on the idea of 'Local Variance' (LV) is employed for this study to obtain optimum scale out of a range of scales. ESP is based on the idea of LV introduced by Woodcock and Strahler (1987). This method was originally utilized to describe pixel resolution of a satellite imagery that optimally represents features of the earth surface. LV is obtained by calculating standard deviation in a small neighbourhood of nxn moving window, then computing the mean over the entire image. Woodcock and Strahler (1987) state that; 'If the spatial resolution is considerably finer than the objects in the scene, most of the measurements in the image will be highly correlated with their neighbours and a measure of local variance will be low. If the objects approximate the size of the resolution cells, then the likelihood of neighbours being similar decreases and the local variance rises. As the size of the resolution cells increase and many objects are found in a single resolution cell, the local variance decreases'. Similarly mean value of within object variation can be utilized to quantify a scale that better represents the scale inherent in the dataset. This idea is evaluated to be adapted for image segmentation (e.g. Kim et al., 2008). Dragut et al (2010) have materialized it as a standard procedure. This tool upon request was made available by the first author and loaded into eCognition software for analysis to obtain appropriate scale for a set of different cases for the study. 
Each case area including; 'case1', 'case1fine', 'case1coarse', 'case1next'and 'case2' were evaluated with ESP tool which produces a set of LV values for successively increasing scale intervals. ESP can evaluate single input at a time, and the authors exercise ESP on slope (Eisank and Dragut, 2009). Therefore particularly slope is exercised for this study. Scale parameter of 1 to 20 is evaluated as this range covers the adequate range of 5 to 12 scales mentioned before. LV value is calculated as the average of all objects' standard deviation. It is expected that LV increases as the objects get larger, however it tends to increase less at some scales which means; although new pixels are included, object's variation doesn't increase that much. As it is not easy to visually interpret the change of LV. Rate of change (ROC) is calculated and plotted. Accordingly, particular scale or scales where the ROC levels out or drops, points out the proper scale that represent the characteristic of the dataset better. ROC plots that are produced for each case are shown. LV and ROC of LV results for the case areas are given in Appendix D. Figure 6.3 represents ROC of LV plot for 'case1 fine', similarly; Figure 6.4 is for 'case1'. Figure 6.5 is for 'case1coarse', and Figure 6.6 is for 'case2'. Arrows denote where there is a level out or tendency of level out.

case1fine

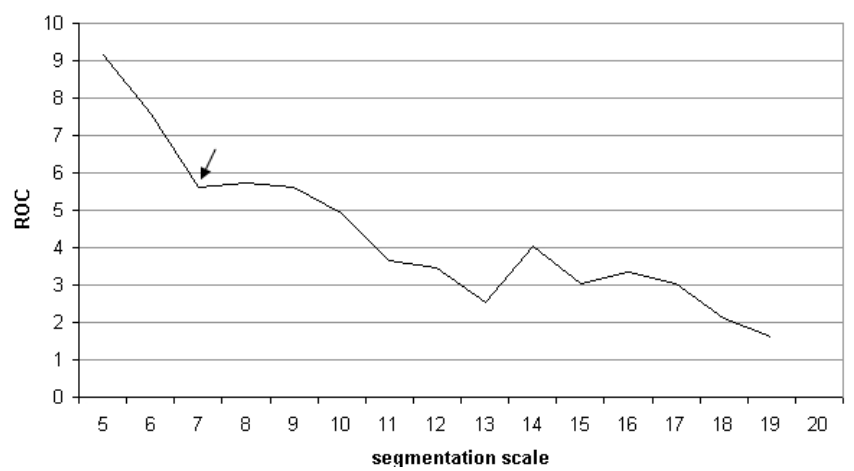

Figure 6.3: Rate of change (ROC) of local variance (LV) of slope calculated over a range of scales (1-20) for 'case1 fine'via ESP tool

case1

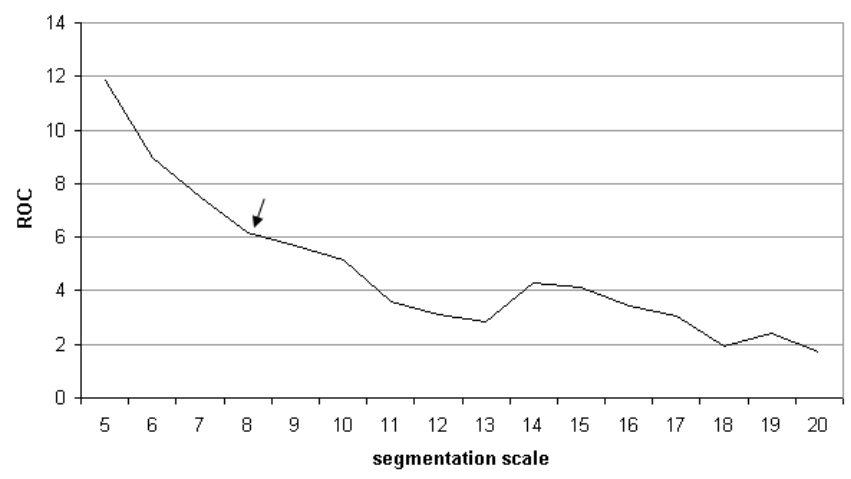

Figure 6.4: Rate of change (ROC) of local variance (LV) of slope calculated over a range of scales (1-20) for 'case1' via ESP tool 


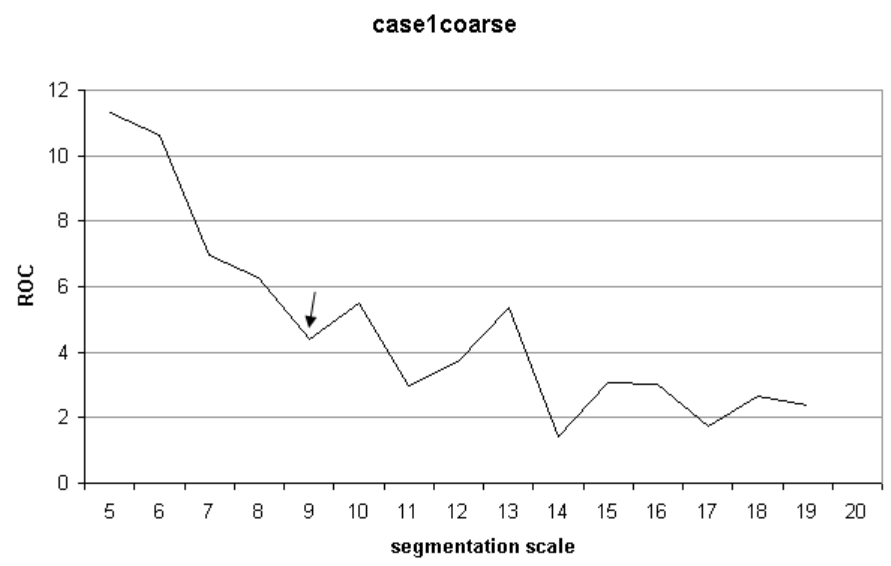

Figure 6.5: Rate of change (ROC) of local variance (LV) of slope calculated over a range of scales (1-20) for 'caselcoarse' via ESP tool

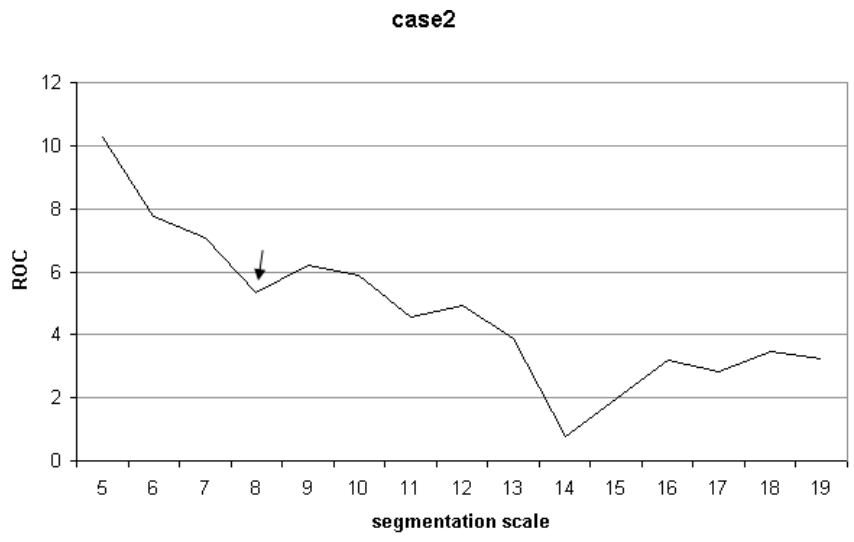

Figure 6.6: Rate of change (ROC) of local variance (LV) of slope calculated over a range of scales (1-20) for "case2", via ESP utility

Scale where ROC curve tends to level out is taken as the optimum scale to be studied. ESP did not pose quite different scales although more difference was expected especially in between 'caselfine', 'case1' and 'case1coarse'. According to the results, scale parameter is taken as 7 for 'case1fine'; 8 for 'case1' and 9 for 'case1coarse'. Overlapping 'case1next' pose almost the same results with 'case1', and scale parameter is taken as ' 8 '. ROC plot yield of 'case2' yield a little bit different from the plots for case1. Scale parameter is set as 8 for 'case2' where the ROC of LV evidently decreases. As all the segmentation parameters are mentioned, parameter set provided for segmentation process is given in Table 6.1. Segmentation results for three datasets; 'case1fine', 'case1', and 'case1 coarse' are given in (Figure 6.7) overlaid on the relief map of the area. A small sample area of $3 \times 3 \mathrm{~km}$ for better visualization is provided with the shaded relief map of 'case1' locating sample area boundary. 
Table 6.1: Segmentation parameters for all cases

\begin{tabular}{|c|c|c|c|c|c|}
\hline Feature & Case1 fine & Case 1 & Case 1 coarse & Case1 next & Case 2 \\
\hline $\begin{array}{l}\text { Segmentation } \\
\text { level }\end{array}$ & \multicolumn{5}{|l|}{ Level -1 } \\
\hline Scale & 7 & 8 & 9 & 8 & 8 \\
\hline color & 0.9 & 0.9 & 0.9 & 0.9 & 0.9 \\
\hline shape & 0.1 & 0.1 & 0.1 & 0.1 & 0.1 \\
\hline
\end{tabular}

It should be regarded that analysis scale of especially for 'case1' and 'caselfine' is reasonably coarser than the relief map used as a base for illustration in Figure 6.7.

Five input layer namely; slope, plan, profile, maximum and minimum curvatures were employed for segmentation at 'Level -1 ' which is the level of morphometry. A set of illustrations that include the morphometric DTMs for the sample area of $3 \times 3 \mathrm{~km}$ with and without relevant segmentation overlaid for different scales of case1 (Figure 6.8, Figure 6.9, Figure 6.10); 'case1 fine', 'case1' and 'case1 coarse' respectively are provided.

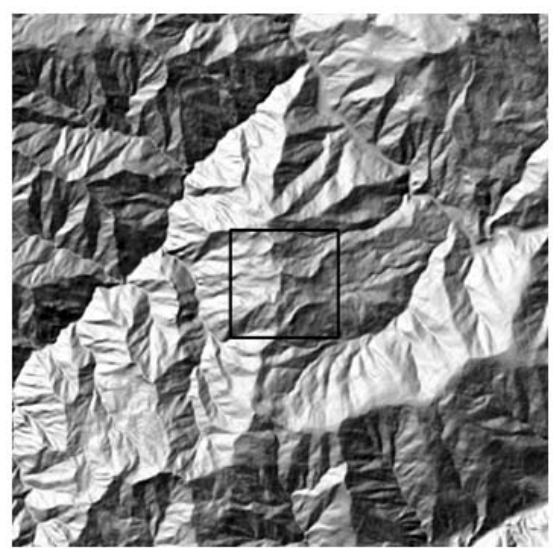

a

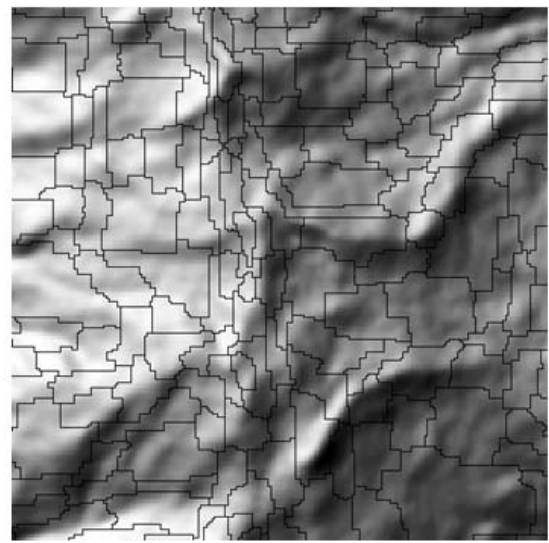

c

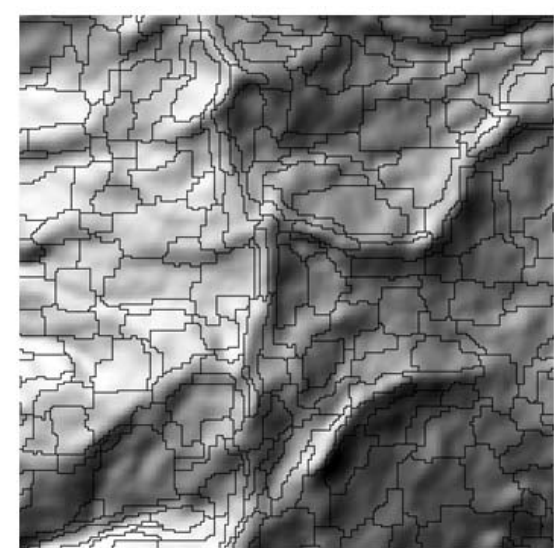

b

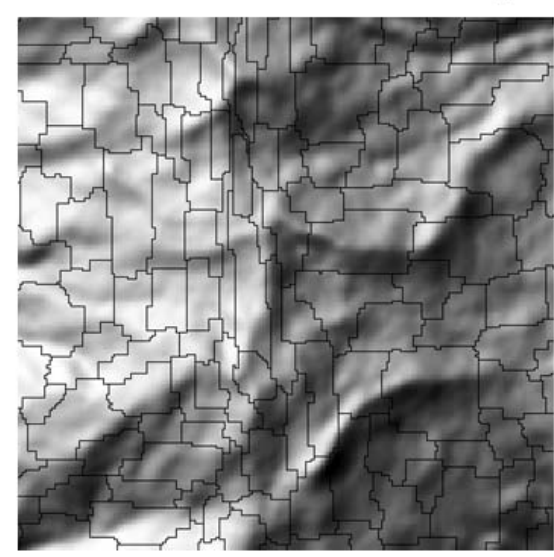

d

Figure 6.7: Relief map of case 1 with a sample area of $3 \times 3$ in the middle (a); segmentation results for 'case1fine' (b); case1 (c), 'case1 coarse' (d) illustrated for the sample area. 

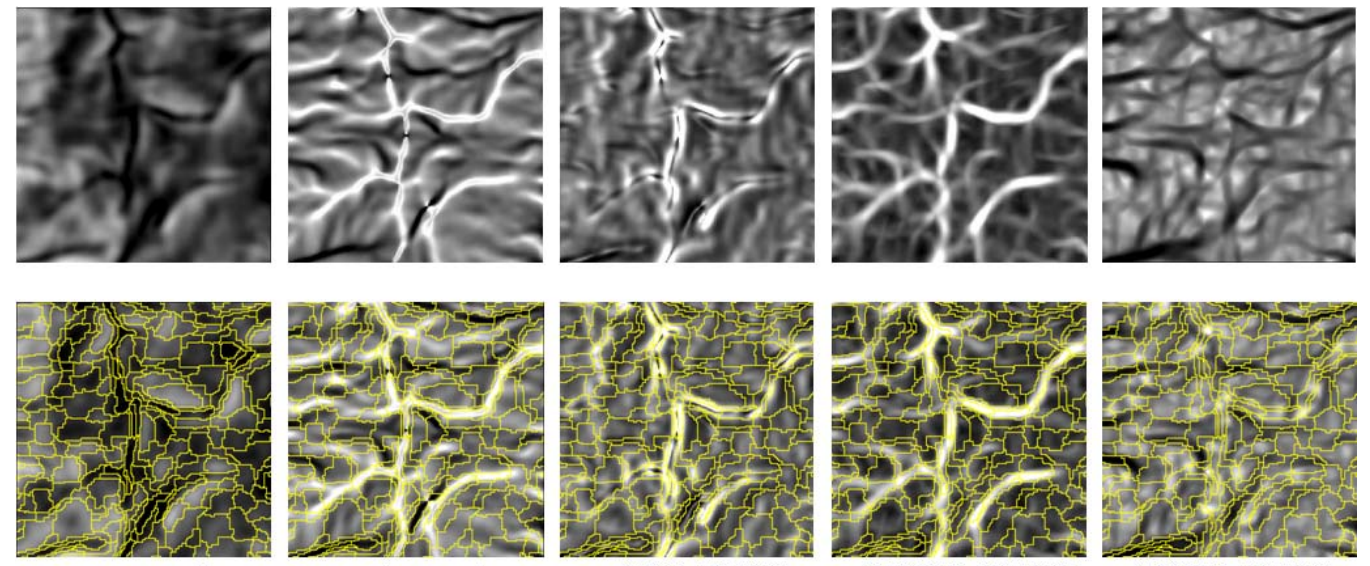

Figure 6.8: Morphometric DTMs of $3 \times 3 \mathrm{~km}$ sample area for 'case1fine' with relevant segmentation overlaid
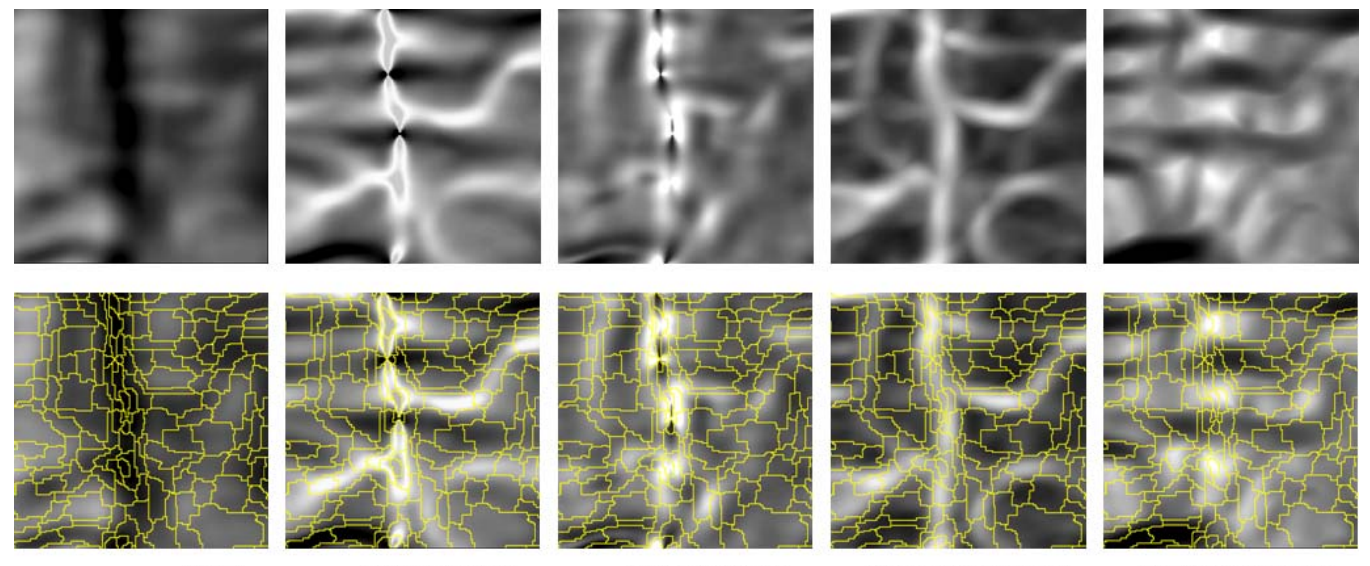

slope

profile curvature

maximum curvature

minimum curvature

Figure 6.9: Morphometric DTMs of $3 \times 3 \mathrm{~km}$ sample area for 'casel' with relevant segmentation overlaid.
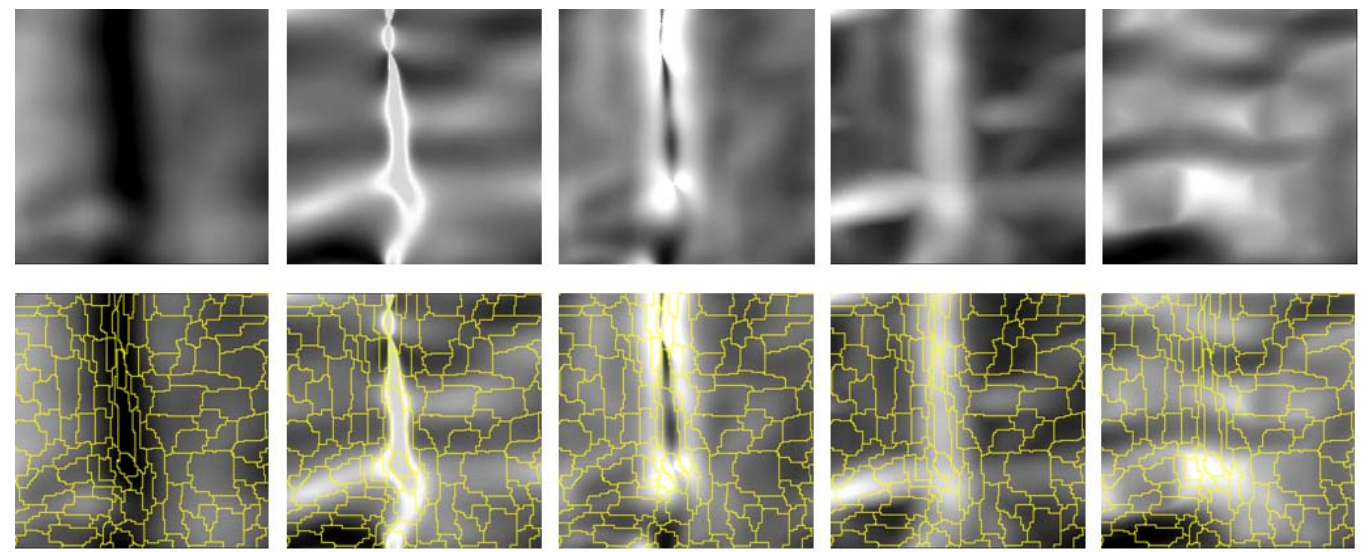

slope

plan curvature

profile curvature

maximum curvature

minimum curvature

Figure 6.10: Morphometric DTMs of $3 \times 3 \mathrm{~km}$ sample area for 'caselcoarse' with relevant segmentation overlaid. 
Segmentation result for 'case2' is given in (Figure 6.11). Segmentation is illustrated for a small sample area of $3 \times 3 \mathrm{~km}$ from the mid part of the scene for better visualization.

DTMs that are employed for segmentation at 'Level -1' for 'case2' and aset of illustrations that include the morphometric DTMs for the sample area of $3 \times 3 \mathrm{~km}$ with and without relevant segmentation overlaid for 'case2' is provided in figure 6.12. Segmentation result for 'case1next' is not illustrated here as the results are quite the same as 'case1'.

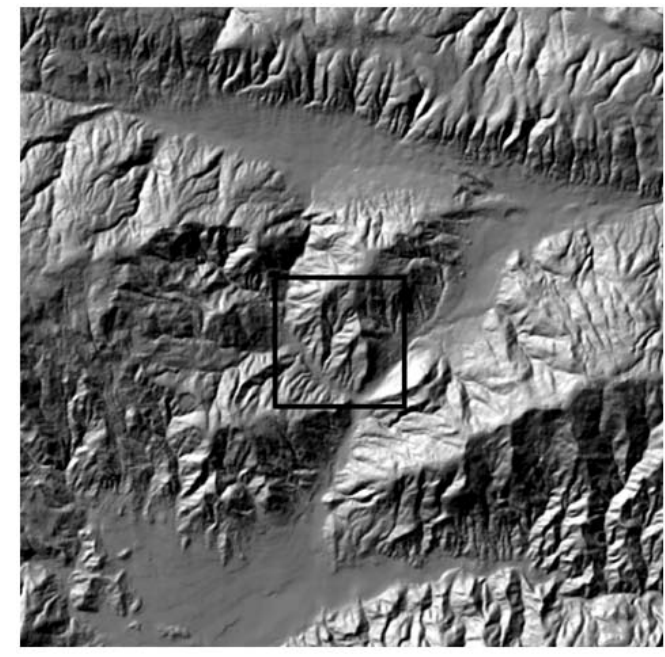

a

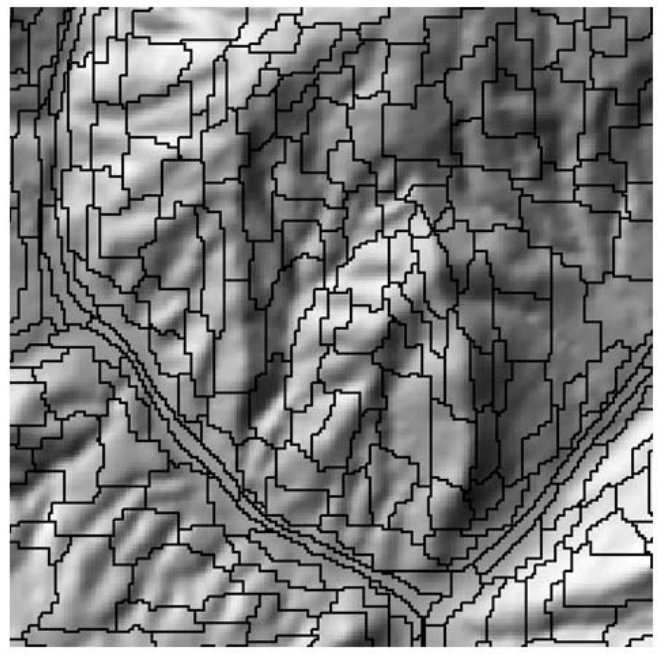

b

Figure 6.11: Relief map of 'case 2 ' with a sample area of $3 \times 3$ in the middle (a) and segmentation results for 'case 2 ' illustrated for the sample area of $3 \times 3 \mathrm{~km}$
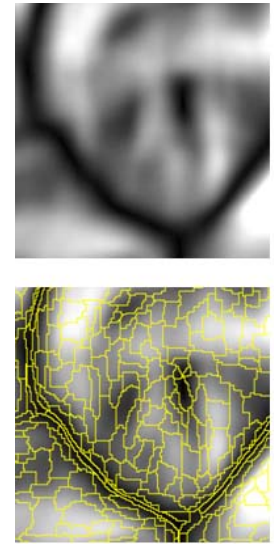

slope
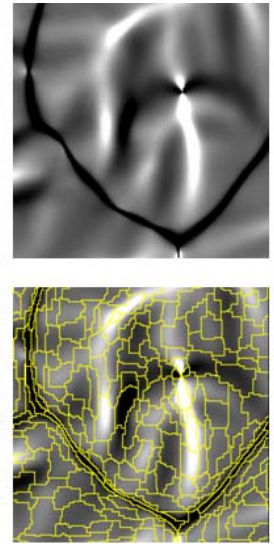

plan curvature
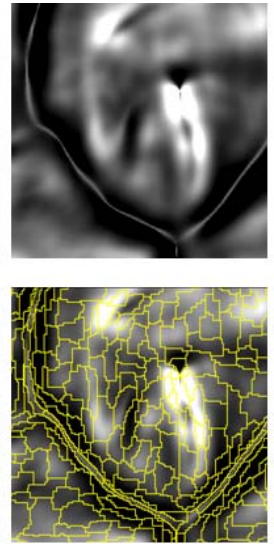

profile curvature
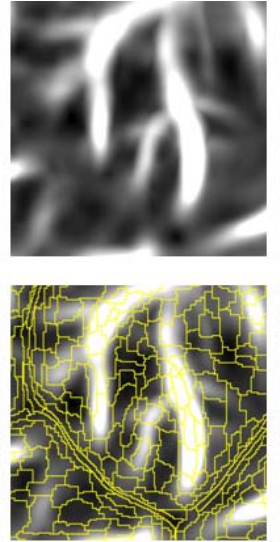

maximum curvature
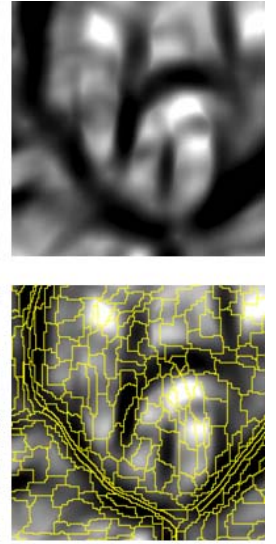

minimum curvature

Figure 6.12: Morphometric DTMs of $3 \times 3 \mathrm{~km}$ sample area in the middle for 'case2' with relevant segmentation overlaid 
After the segmentation on the lower level: 'Level -1 ' is implemented, other levels are produced out of it. Higher level segmentations are produced using initial segmentation based on form (Level -1). Image objects from 'Level -1' are duplicated and they are put on the higher levels of hierarchy, i.e. 'Level 0' and 'Level 1'. These levels are then utilized in geomorphometric classification as mentioned in section 6.2.3.

\subsection{Object-based classification}

Classification for the study mainly comprises of two parts; initially, objects gathered out of segmentation of morphometric DTMs are classified based on local geometry, and 'morphometric classes' are obtained. Then, 'morphometric classes' and geomorphometric context that is represented with 'relative terrain position' and 'terrain network' are all utilized together to end up with 'geomorphometric classes'. The procedure is actually reclassification of 'morphometric classes' regarding geomorphometric context through a set of logical operators to yield 'geomorphometric classes' utilizing multi-level hierarchy of object levels.

\subsubsection{Classification of landforms based on local geometry}

A fuzzy classification is implemented that well represent the continuous nature of terrain for classification of landforms based on local geometry / form. Among the two methods of implementing fuzzy classification; 'semantic import (SI) model' is employed to describe class concepts for landform classes. SI models that utilize fuzzy membership functions require two parameters to be defined; (i) type of curves (e.g. linear, bell shaped, z-shaped, sshaped (Figure 6.12) and (ii) threshold values (a, b in Figure 6.13).

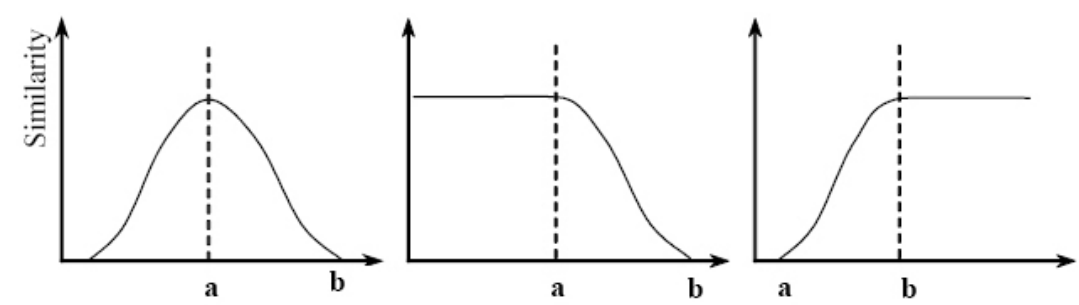

Figure 6.13: Bell shaped, $\mathrm{z}$-shaped, s-shaped membership functions, a and b are the threshold limits.

Threshold values ' $a$ ' and ' $b$ ' are the values for particular criteria describing an entity of a class where membership of belonging to it reaches is 1 at ' $a$ ' and decreases to 0 at ' $b$ '. A 'cross over' point in a membership function is also of critical importance. This value is somewhere in between ' $a$ ' and ' $b$ ' and corresponds to data value where membership gets 
0.5. When two membership functions are evaluated together e.g. steepness and flatness; cross over is the corresponding value of intersection point between ' $a$ ' and ' $b$ ' that represent absolute flatness or steepness. Cases with values higher than cross over are assigned to a class, and lower than that are assigned to the other.

ECognition provides a set of membership functions; both fuzzy and crisp that can be utilized in classification (Figure 6.14). Among the provided set of membership functions 'linear larger than', 'linear smaller than' and Approximate Gaussian' functions are employed. Linear function and simple Gaussian approximate functions are utilized for describing membership functions for this study (Figure 6.15).

\begin{tabular}{|c|c|c|c|}
\hline \multicolumn{2}{|c|}{ Fuzzy } & \multicolumn{2}{|c|}{ Crisp } \\
\hline Function Slope & Description & Function Slope & Description \\
\hline & Larger than & & $\begin{array}{l}\text { Larger than } \\
\text { (boolean, crisp) }\end{array}$ \\
\hline & Smaller than & & $\begin{array}{l}\text { Smaller than } \\
\text { (boolean, crisp) }\end{array}$ \\
\hline & Linear larger than & & $\begin{array}{l}\text { Singleton (single } \\
\text { exact value) }\end{array}$ \\
\hline & Linear smaller than & & Full range \\
\hline & Linear Range (V) & & \\
\hline & $\begin{array}{l}\text { Linear Range } \\
\text { (inverted V) }\end{array}$ & & \\
\hline & About Range & & \\
\hline$\Lambda$ & $\begin{array}{l}\text { Approximate } \\
\text { Gaussian }\end{array}$ & & \\
\hline
\end{tabular}

Figure 6.14: Set of membership functions that can be utilized in classification in eCognition software (eCognition Developer v.8, Reference Book).

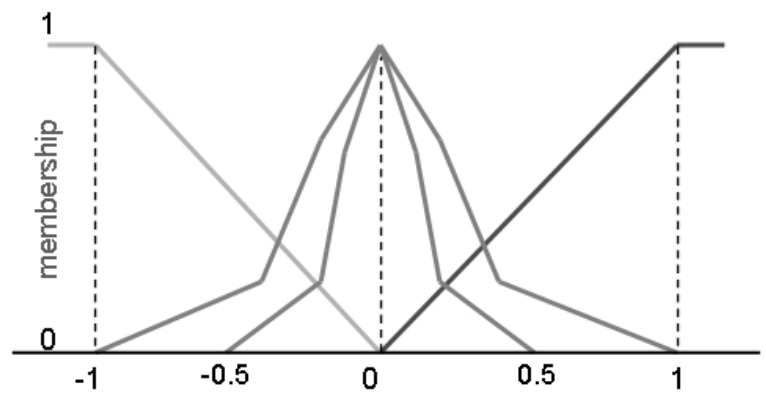

Figure 6.15: Fuzzy membership functions used as Semantic Import (SI) model for the study. 
Linear functions are used for importing semantics for flatness vs. steepness in slope and curvedness for curvatures, straightness (no curvature) is represented with approximate Gaussian function as the frequency distribution of curvature DTMs are bell-shaped with most of the values clustered around 0 , therefore better represented using a simple Gaussian curve, a narrow version is employed to describe straightness in planar forms; 'plain' and 'planar slope'. Using simple functions satisfy simplicity constraints and important for reproducibility. Another reason for preferring simple membership functions is relative ease to construct a look-up table to evaluate membership values and their corresponding original DTM values if needed.

Slope and curvature are descriptors of form. Slope is used to quantify whether the topography is flat or sloping. Sloping areas are then subdivided into nine classes regarding their plan and profile curvatures, and flat areas are subdivided into six classes regarding their minimum and maximum curvatures pertaining to the criteria whether they are convex, straight or concave (Figure 2.2) (Dikau 89). The question then arises; what is the extent to which a form is sloping, or a form is curved. Unfortunately there's no explicit and satisfactory criteria defined for general geomorphometric purposes, there are vast amount of thresholds described for slope that cluster around 30 (Giles, 1998; MacMillan and Shary, 2004; Speight, 1990; Reuter et al., 2006) which mainly rely on field observations. Curvature on the other side is very loosely defined to describe convex or concave features across the landscape especially for general purposes. Dikau (1989) provides a curvature measure for his scheme where values lower than of $600 \mathrm{~m}$ radius that is; $0.16 / 100 \mathrm{~m}$ represent uniformity and values higher represent curvedness. Reuter et al. (2006) suggested $0.1 / 100 \mathrm{~m}$ for curvedness. Considering a data range of curvatures obtained e.g. 3.0 to $5.9 / 100 \mathrm{~m}$ for case1, these values remain very small (close to straightness) to be used as a 'crossover' in this study. If a crossover value of $0.1 / 100 \mathrm{~m}$ is defined, than all the values higher than that should be assigned to a curved class, which correspond to about $90 \%$ of case 1 . Therefore a higher curvature value that better fits data is evaluated. Coops et al. (1998) defined $>0.5$ and $<$ $0.8 / 100 \mathrm{~m}$ plan curvature for crests and depressions respectively and that seems quite high as those values are typically used for extracting local minima and maxima. A moderate value should be obtained to be used a s cross over for fuzzy descriptions.

Other important issue to be considered is that; those thresholds can not be accepted as absolute values, as window of perception has an influence on characteristics of the terrain and DTM values tend to change with enlarging window sizes that are used to calculate them. Assuming that one produced a set of DTMs using enlarging windows to classify 
landforms at specific scale or for specific phenomena, thresholds for each DTM should be adjusted to get a similar output, e.g., Reuter et al. (2006) employed a non-linear parameter optimisation algorithm to adjust thresholds for a range of DTMs derived at different scales to classify several landforms. Moreover those thresholds can not be considered global; a threshold that yields good results in a particular area is less likely to be that successful in another. Case areas were examined to obtain threshold values for slope and curvature DTMs considering scale associated with the window size. Some tentative slope and curvature measures to discriminate convex, straight and convex features could be observed. However they were quite different in value for the two areas, which would necessitate different procedures for different cases where the study would lack constraints for reproducibility and repeatability.

Considering ambiguity in of thresholds for landform classification in the literature, and need to adjust thresholds for different scales or different area; it is suggested that normalized DTMs can be a solution for landform classification of general geomorphometry. Accordingly, slope and curvature values are normalized so that they represent general steepness or general curvedness of any landscape.

For determination of 'threshold' values, empirical, visual and statistical measures are put forward. Statistical measures are quantitative and they can represent relativity of populations (case areas for this study) that have different characteristics for a set of measured properties. At this point summary statistics were involved. Maximum, minimum and mean values for the DTMs of all the cases are given in Table 6.2.

Table 6.2: Summary statistics for morphometric DTMs of case areas: 'caselfine', case1, 'case1coarse', 'case1next', 'case2'.

\begin{tabular}{|c|c|c|c|c|c|c|}
\hline Feature & case1fine & case1 & case1coarse & case1next & case 2 & case 3 \\
\hline $\begin{array}{l}\text { Slope } \\
\left({ }^{0}\right)\end{array}$ & $\begin{array}{l}\min : 0 \\
\max : 53.2 \\
\text { mean: } 27.1\end{array}$ & $\begin{array}{l}\min : 0 \\
\text { max: } 40.8 \\
\text { mean: } 22.3\end{array}$ & $\begin{array}{l}\min : 0 \\
\text { max: } 34.7 \\
\text { mean: } 19.4\end{array}$ & $\begin{array}{l}\text { min: } 0 \\
\text { max: } 40.7 \\
\text { mean: } 22.2\end{array}$ & $\begin{array}{l}\text { min: } 0 \\
\text { max: } 28.5 \\
\text { mean: } 9.5\end{array}$ & $\begin{array}{l}\text { min: } 0 \\
\text { max: } 4 \\
\text { mean: } 1.08 \\
\end{array}$ \\
\hline $\begin{array}{l}\text { Plan } \\
\text { Curvature } \\
(/ 100 \mathrm{~m})\end{array}$ & $\begin{array}{l}\min :-10 \\
\max : 10\end{array}$ & $\begin{array}{l}\min :-10 \\
\max : 10\end{array}$ & $\begin{array}{l}\min :-10 \\
\max : 10\end{array}$ & $\begin{array}{l}\min :-10 \\
\max : 10\end{array}$ & $\begin{array}{l}\min :-10 \\
\max : 10\end{array}$ & $\begin{array}{l}\min :-10 \\
\max : 10\end{array}$ \\
\hline $\begin{array}{l}\begin{array}{l}\text { Profile } \\
\text { Curvature } \\
(/ 100 \mathrm{~m})\end{array} \\
\end{array}$ & $\begin{array}{l}\min :-4.0 \\
\max : 2.8\end{array}$ & $\begin{array}{l}\min :-3.4 \\
\max : 2.6\end{array}$ & $\begin{array}{l}\min :-2.5 \\
\max : 1.9\end{array}$ & $\begin{array}{l}\min :-3.3 \\
\max : 2.6\end{array}$ & $\begin{array}{l}\min :-1.5 \\
\max : 1.4\end{array}$ & $\begin{array}{l}\min :-0.17 \\
\max : 0.16\end{array}$ \\
\hline $\begin{array}{l}\text { Maximum } \\
\text { Curvature } \\
(/ 100 \mathrm{~m})\end{array}$ & $\begin{array}{l}\min :-9.1 \\
\max : 3.4\end{array}$ & $\begin{array}{l}\min :-3.0 \\
\max : 5.9\end{array}$ & $\begin{array}{l}\min :-2.1 \\
\max : 4.7\end{array}$ & $\begin{array}{l}\min :-3.2 \\
\max : 5.4\end{array}$ & $\begin{array}{l}\min :-1.25 \\
\max : 3.3\end{array}$ & $\begin{array}{l}\min :-0.19 \\
\max : 0.32\end{array}$ \\
\hline $\begin{array}{l}\text { Minimum } \\
\text { Curvature } \\
(/ 100 \mathrm{~m})\end{array}$ & $\begin{array}{l}\min :-3.1 \\
\max : 7.8\end{array}$ & $\begin{array}{l}\min :-7.0 \\
\max : 3.2\end{array}$ & $\begin{array}{l}\min :-5.3 \\
\max : 2.3\end{array}$ & $\begin{array}{l}\min :-7.6 \\
\max : 3.2\end{array}$ & $\begin{array}{l}\min :-4.0 \\
\max : 1.99\end{array}$ & $\begin{array}{l}\min :-0.34 \\
\max : 0.16\end{array}$ \\
\hline
\end{tabular}


Defining membership function parameters for curvature: Curvature DTMs have values that range in between $(-)$ and $(+)$ where 'negative' values denote convexity, 'positive' values denote concavity and ' 0 ' denotes no curvature or straightness.

Use of original minimum and maximum values of curvature is considered inappropriate to be used as bounding limits for normalization procedure. Because, minimum and maximum values are observed to be outliers and direct use of them would lead misclassifications such as; maximum value that is assigned as 1 after normalization would be so high that image objects can hardly satisfy it to be assigned as a curved class i.e. peak. Hence, there should be a value of curvature for which all the values higher than that are assigned as curved. This value is gathered by trimming of the outliers at a specific rate of percentile. Actually this operation is called histogram winsorising. In image processing context; histogram trimming process totally removes outliers and those regions that correspond to outliers are masked. Winsorization however is more like a transformation in which one replaces the outliers with a threshold quantile or percentile value. For the study curvature DTM histograms are winsorised the by $\% 5$ percentile, so that $\% 5$ of the values are assigned with the value that correspond to $95^{\text {th }} \%$ percentile. That value represents the maximum value of curvedness.

For each curvature value, minimum and maximum values are replaced with relevant percentile values. Then they are linearly normalized into a range of -1 to +1 with a series of operations employing raster calculator utility of ArcGIS given in detail in Appendix E.1. Percentile values are obtained utilizing TNTmips and MS excel. Membership functions that are used to import semantics for curvature are given in Figure 6.16.

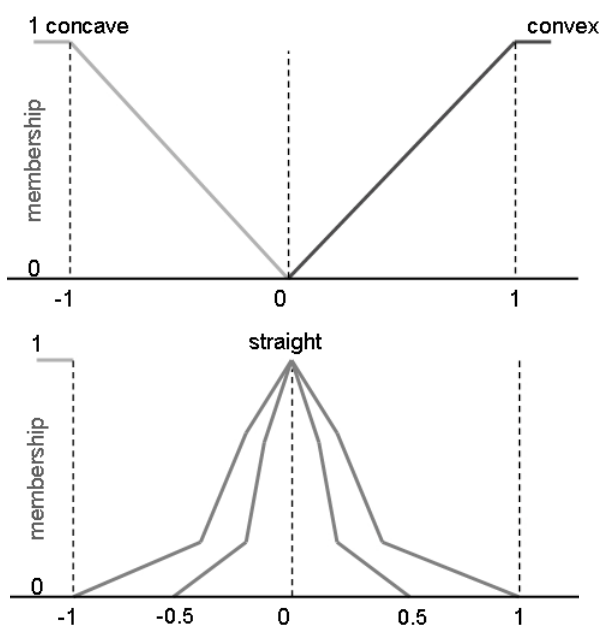

Figure 6.16: Membership functions that are used to import semantics on curvedness (top) and straightness (bottom). 
Defining membership function parameters for slope: Slope is quite different in characteristics compared to curvature DTMs. They range in between ' 0 ' that corresponds to flatness and a positive value up to $90^{\circ}$ or $100 \%$ which is not likely to be observed naturally. However, it is troublesome to set a constant threshold value for slope that describes steepness and that applies to any area of interest. Since this study proposes a landform classification of general geomorphometry and it is not intended for a specific purpose, a relative measure based on summary statistic is offered, that is 'mean' slope. Mean value is arithmetic average of all values of a population (slope for the case). Therefore it provided a measure of class central concept; values that are higher than the mean slope are accepted as 'steep'.

However one should consider amount of flat values when extracting a mean value out of a slope raster. Flat areas with values near zero would shift the mean towards lower values and influence the proper characterisation of threshold value. Those areas that are flat should be disregarded when computing a mean slope of the whole area. This is not a problem in 'case1' where flat areas constitute only a very small portion of the area. However 'case2' has flat areas that should be eliminated before extracting a slope threshold. In this context, flat areas are considered to be areas lower than $3^{0}$ as suggested by many authors and lower slopes are eliminated, and then mean slope is calculated. This value is 22.42 for 'case1' and it would be 22.3 if the slope was calculated for the whole area, it is 10.6 for 'case2' and it would be 9.5 if the slope was calculated for the whole area, difference is higher in 'case2' as there is more amount of flat topography. Obtained mean values are then used to winsorize histogram for higher slope values that represents the maximum value of steepness. Then these winsorised rasters are linearly normalized into range of 0 to +1 with a series of operations employing raster calculator utility of ArcGIS given in detail in Appendix E.2. Membership functions that are used to import semantics for slope are given in Figure 6.17. Normalization process is performed for all of the dataset that is used as input for classification.

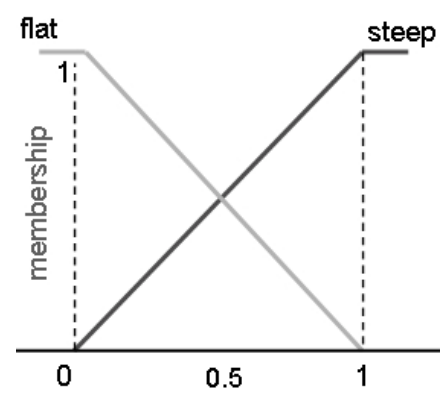

Figure 6.17: Membership functions that are used to import semantics on steepness vs. flatness. 
Fuzzy membership functions used in the study returns values between the ranges of 0 to 1 for belonging to a particular class. As mentioned earlier, form is represented by slope and curvature where objects with low slope are classified into six non-sloping elements according to maximum and minimum curvature. Therefore, to describe non-sloping forms three DTMs are utilized; slope, maximum curvature and minimum curvature, similarly sloping elements that are comprised of nine forms are described by; slope, plan and profile curvature using fuzzy membership functions for each. There are various options to operate values returned from each membership function. A list of them provided in eCognition is given in Table 6.3.

Table 6.3: A list of operators to utilize information from several membership functions (eCognition Developer v.8, Reference Book).

\begin{tabular}{l|l} 
Operator & Description \\
\hline or (max) & $\begin{array}{l}\text { or-operator returning the maximum of the fuzzy values, the } \\
\text { strongest or }\end{array}$ \\
\hline mean (arithmetic) & arithmetic mean of the fuzzy values \\
\hline mean (geo.) & $\begin{array}{l}\text { and-operator returning the minimum of the fuzzy values (used } \\
\text { by default, the most reluctant and) }\end{array}$ \\
\hline and (*) & geometric mean of the fuzzy values \\
\hline not & and-operator returning the product of the fuzzy values \\
\hline
\end{tabular}

In this study 'mean' operator that returns the equally weighted average of the membership values from three criteria set is employed (Figure 6.18).

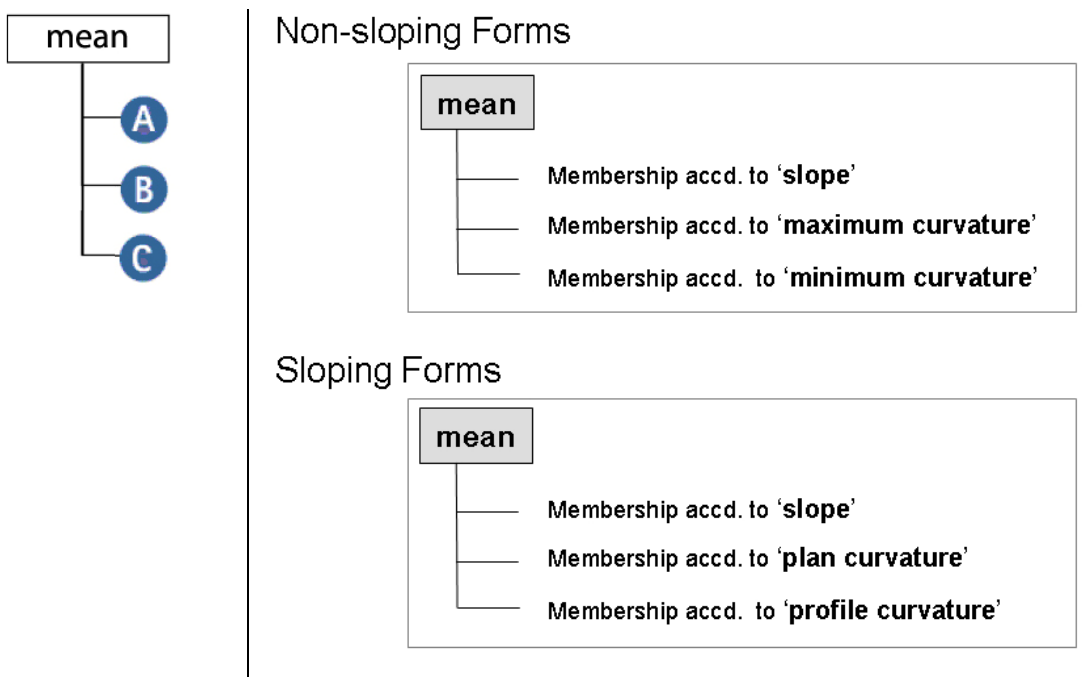

Figure 6.18: Schematic representation of membership operator of form descriptors employed for the study. 
Accordingly, A, B, and C are descriptor characteristics of classes. They return values between 0 and 1, regarding degree of fit of an object with the characteristic described. Mean of these membership values return an average value of degree of membership for each class. Object is assigned to the class for which the membership value gets the highest.

To avoid full descriptions of each class, membership functions that are utilized in SI model are presented. Accordingly; a set of three rules were described for 15 morphometric classes; first six of the rules pertain to non-sloping forms (Figure 6.19), other nine set of rules pertain to sloping forms (Figure 6.20). Same membership function of slope is employed for classes within each group of forms.

Accordingly; a peak for instance is described with its non-sloping topography. Membership function for slope returns values close to 1 if the surface form at a specific location is flat. Membership functions for maximum and minimum curvature returns high values close to 1 where the surface has convexity in maximum and minimum curvature. If the average value for all three criteria returned highest for class 'peak', then this object is classified as 'peak'. Moreover object also contains information on belonging to each of the classes given, which constitutes the main idea of fuzzy classification.

Morphometric classification is performed on all case areas. However, it has been applied on case1 for three different scales denoted as 'case1fine', 'case1' and 'case1coarse'. Following three figures (Figure 6.21, Figure 6.22 and Figure 6.23) are morphometric classification results for these three scales of cases respectively.

Figure 6.24 zooms into the sample $3 \times 3 \mathrm{~km}$ rectangle in the middle of 'case1' and illustrates the classes produced in different scales. It can be clearly observed that classes get more generalized as scale gets coarser. Classes tend to be replaced with other classes where some classes are more likely to stay. For instance, in 'case1fine' sharp ridges at the eastern part of the main ridge are classified as ridge, in 'case1' they were replaced with wider spurs and in 'case1coarse' they disappeared and have been classified as planar slopes. However, main ridge that is in north-south direction although with discontinuities can be observed in three of the classifications. 


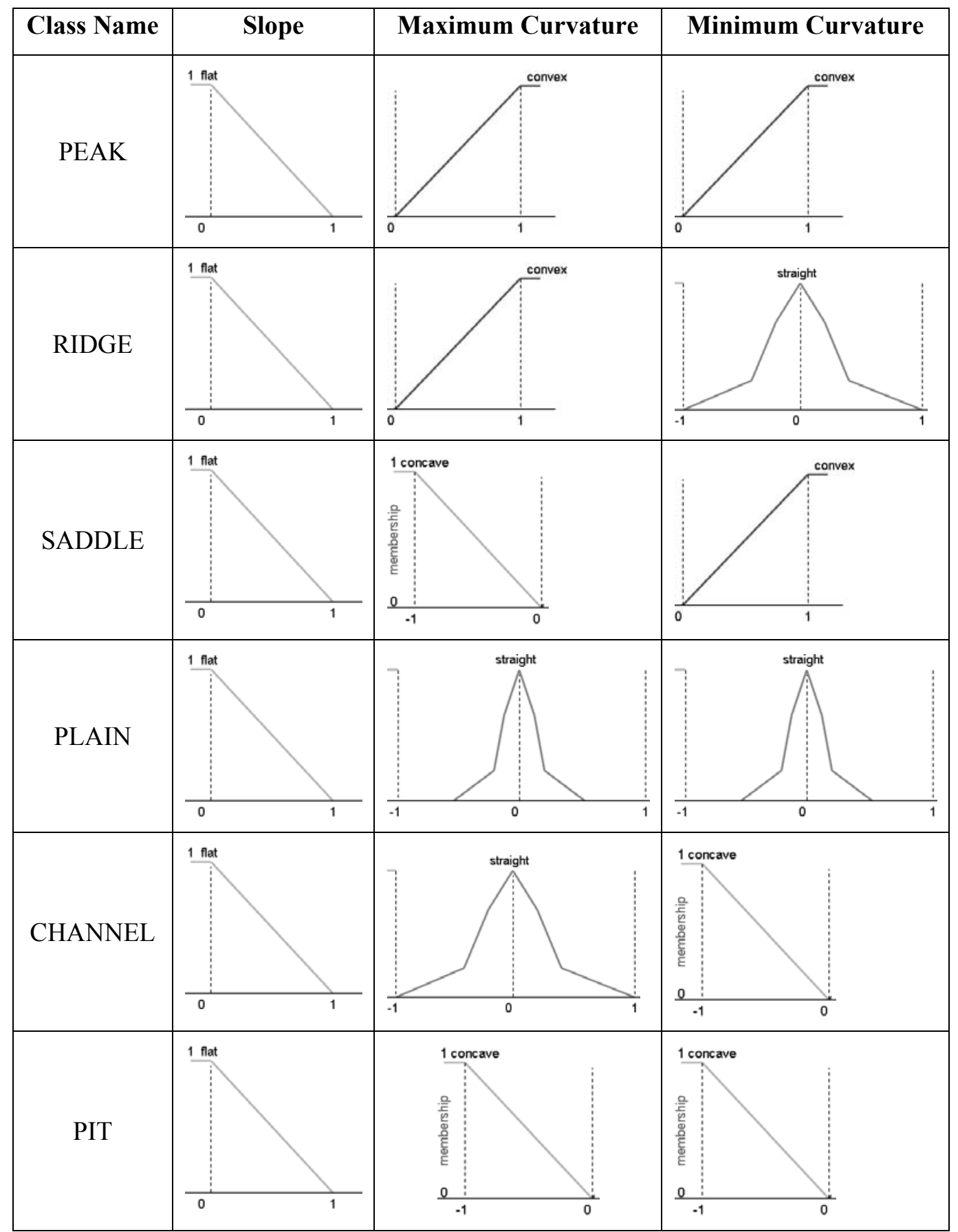

Figure 6.19: Fuzzy membership functions used for describing non-sloping forms 


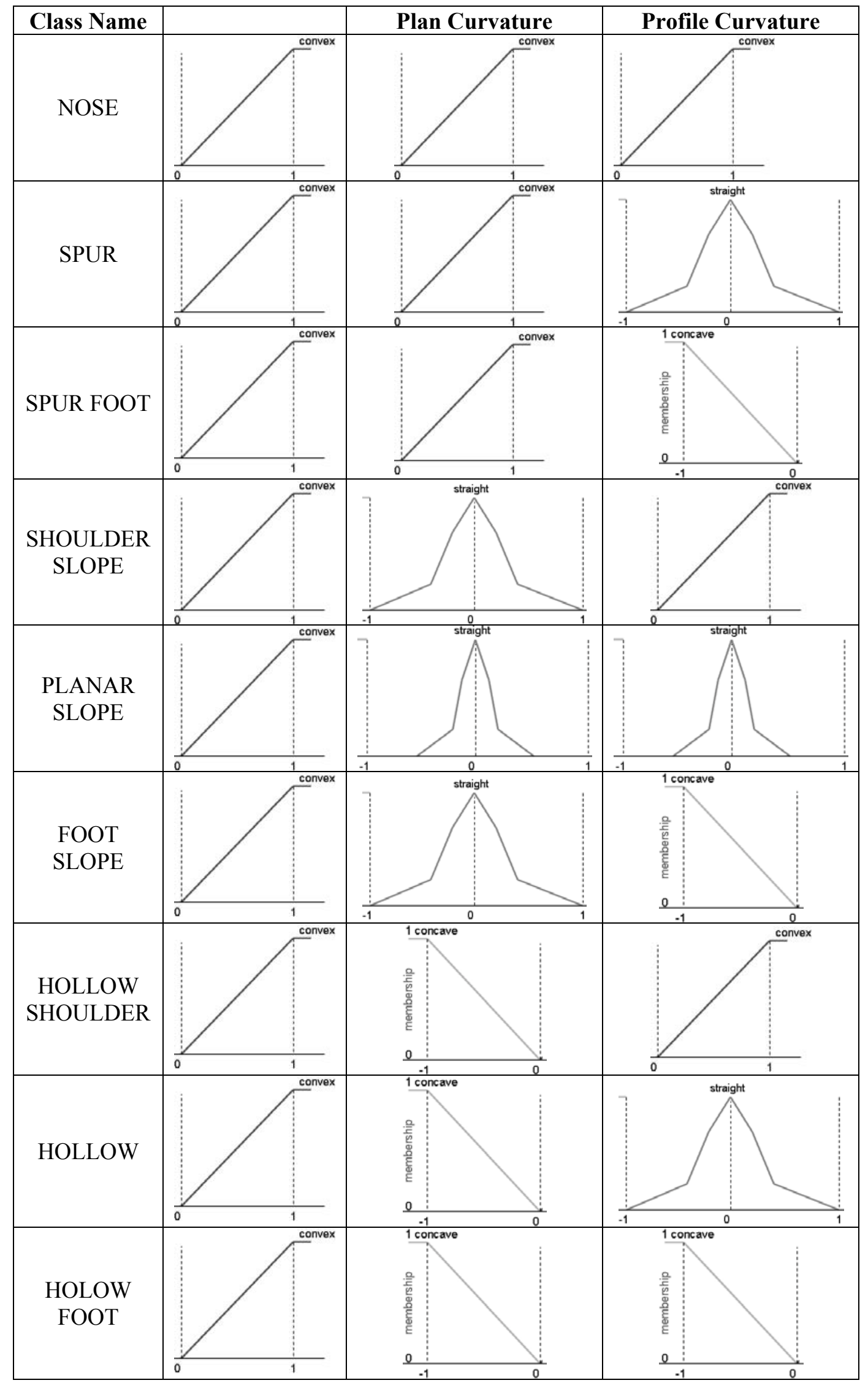

Figure 6.20: Fuzzy membership functions used for describing non-sloping forms. 


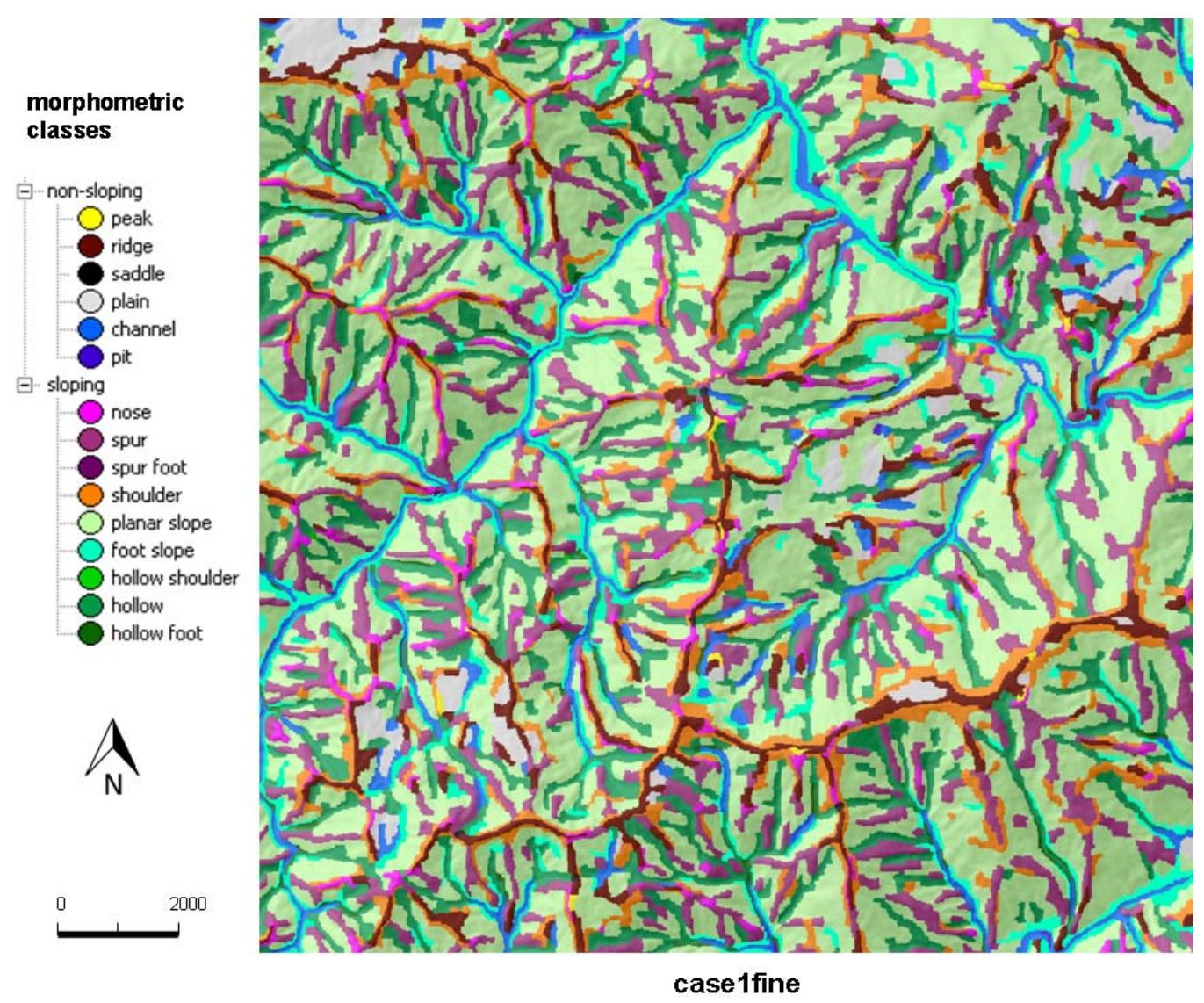

Figure 6.21: Morphometric classification result for 'case1 fine'. 


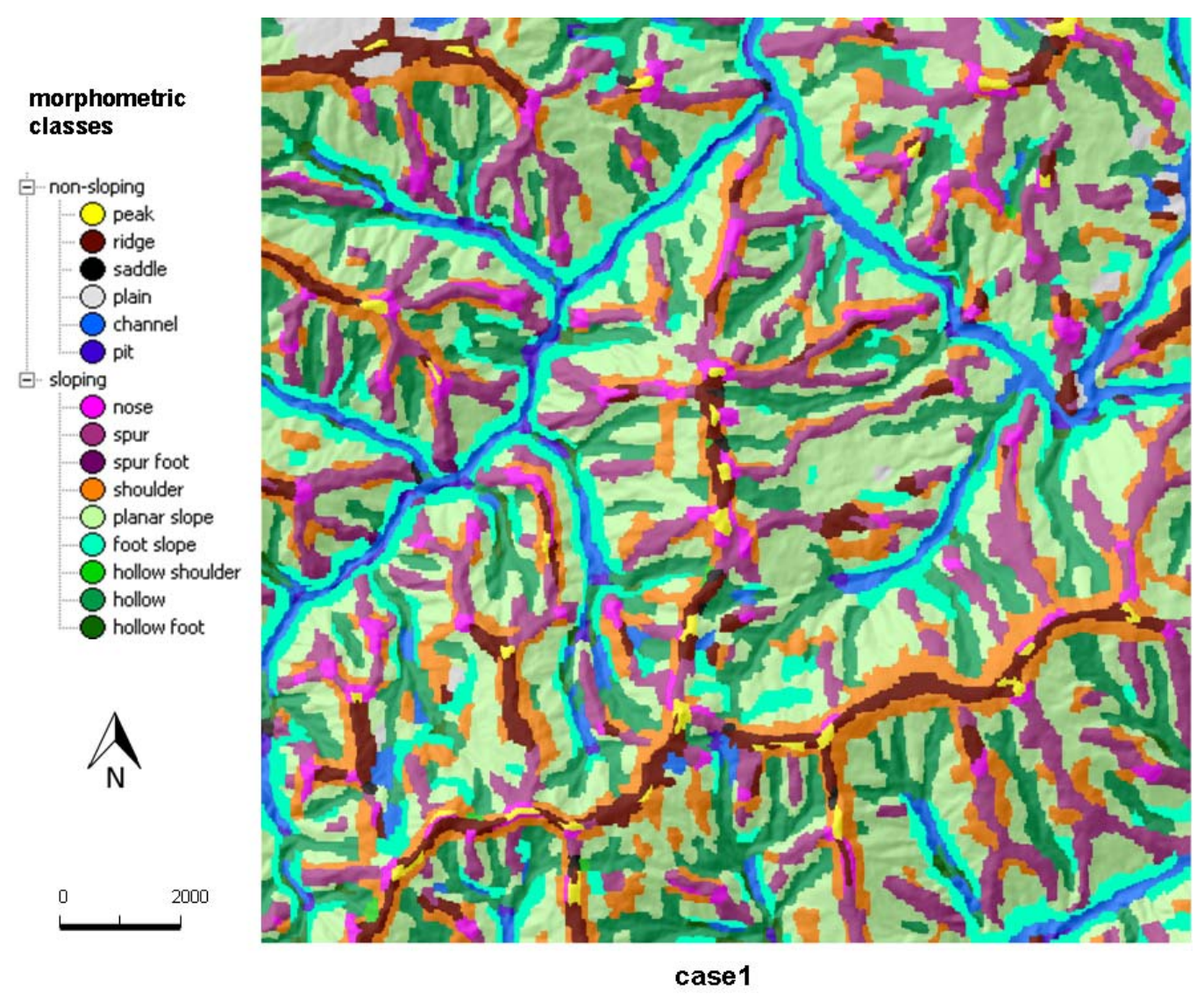

Figure 6.22: Morphometric classification result for 'case1'. 


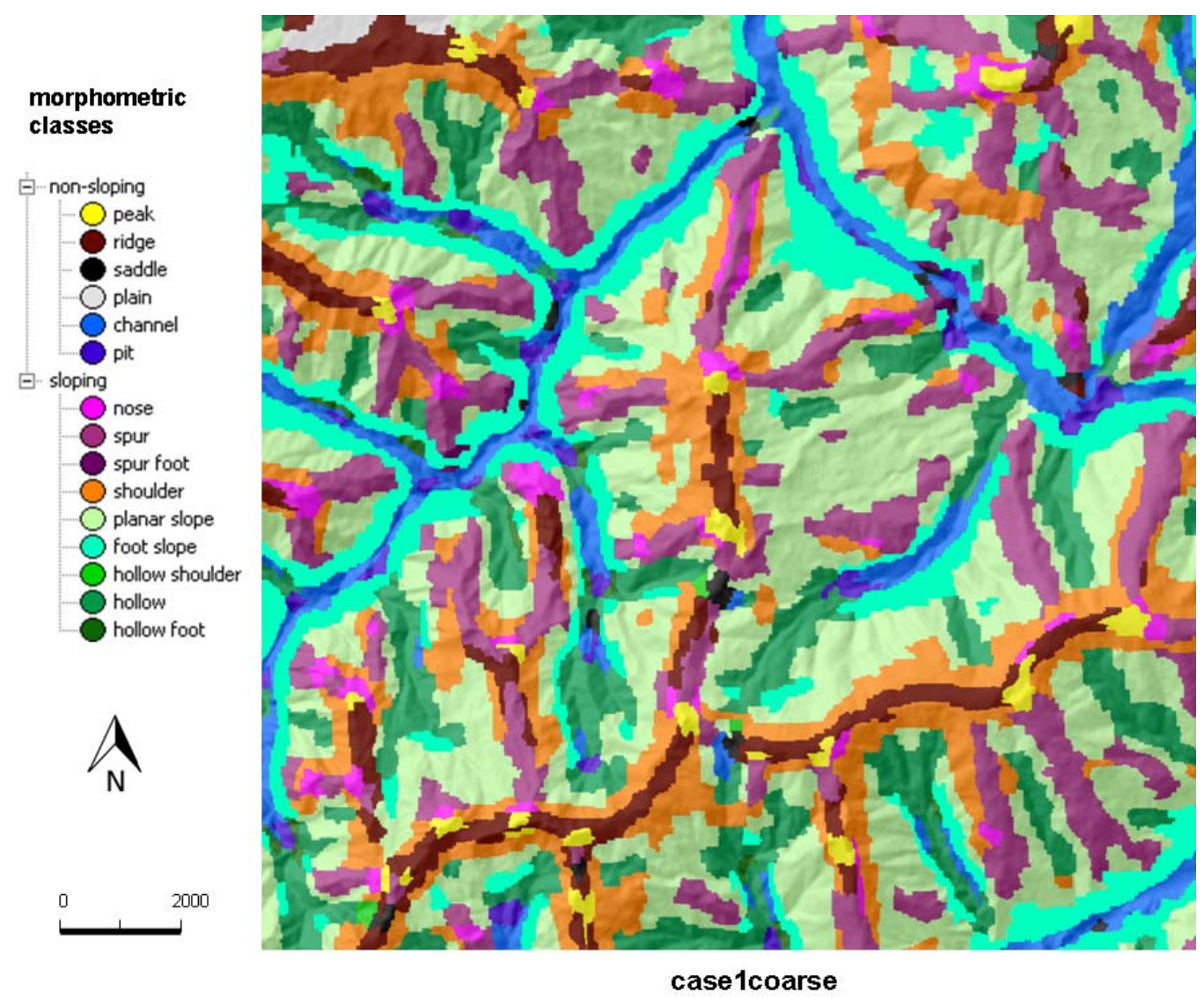

Figure 6.23: Morphometric classification result for 'casel coarse'. 


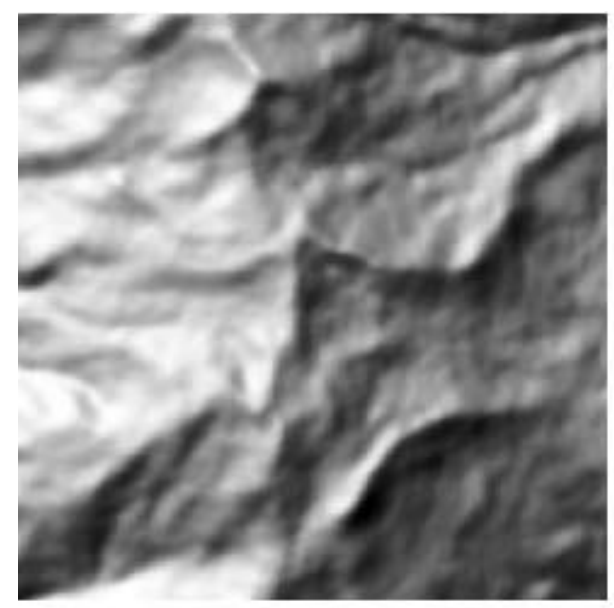

morphometric

classes

$\square$-non-sloping

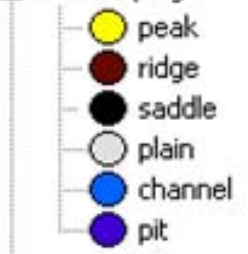

- sloping

nose
spur
spur foot
shoulder
planar slope
foot slope
hollow shoulder
hollow
hollow foot

$\mathrm{N}_{\mathrm{N}}$

0

3000
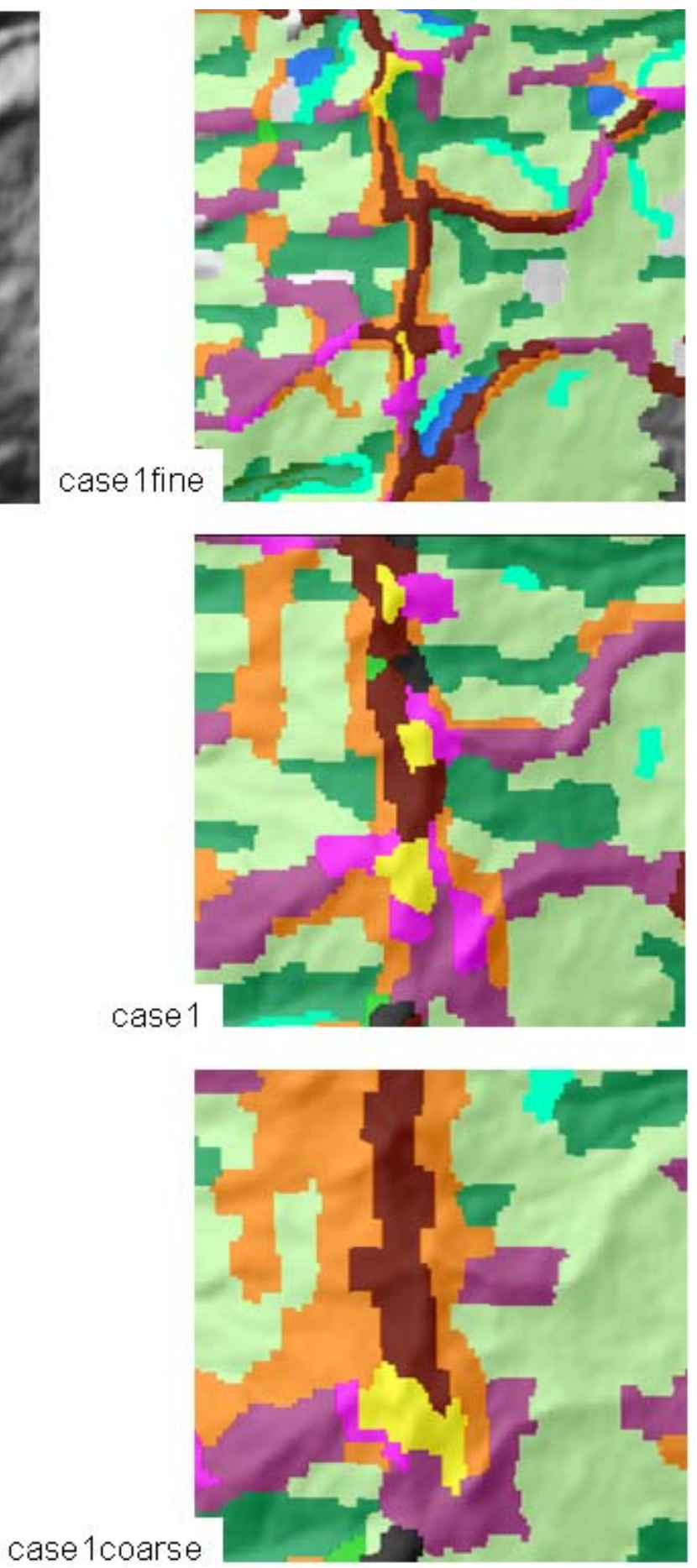

Figure 6.24: Morphometric classification result for $3 \times 3$ sample area for 'case1fine', 'case1' and 'case1 coarse'. 


\subsubsection{Classification based on terrain position}

In order to describe relative position of forms across the landscape Topographic Position Index (TPI) which is a 'local elevation' based method is employed. TPI compares altitude of the center pixel of a window with the mean elevation of that window and produces relative values in a range of (-) to $(+)$ where positive TPI values represent locations that are higher than the average of the local window e.g. ridges. Negative TPI values represent locations that are lower e.g. valleys.

Minimum and maximum TPI values that are calculated for the cases to be utilized in geomorphometric classification are given in Table 6.4. 'Case1fine' and 'case1coarse' are omitted from the remaining analysis.

Table 6.4: Topographic Position Index (TPI) value ranges for case areas: 'case1', 'caselnext' and 'case2'.

\begin{tabular}{llll}
\hline Feature & case1 & case1next & case2 \\
\hline \multirow{2}{*}{ TPI } & $\min :-448$ & $\min :-420$ & $\min :-201$ \\
& $\max : 504$ & $\max : 503$ & $\max : 227$ \\
\hline
\end{tabular}

TPI for 'case1' and 'case1next' are quite similar where as TPI for 'case2' is considerably low compared to them. This is due to relatively smaller amount of change in relief in 'case2' compared to 'case1' areas. Direct use of these values will require particular adjustments on rules for each case which is avoided. Therefore similarly as morphometric DTMs, original TPI values are normalized. TPI values depict a bell-shaped distribution frequency clustered around 0 . As suggested by Jenness (2006) who has developed a tool that can be embedded into ArcView, TPI values are standardized first. However they did not pose similar ranges and therefore further linearly normalized into a range 0 to 1 .

Normalized TPI values are categorized into three as 'upper', 'mid', and 'lower' using crisp rules. Suggested threshold for class descriptions by Jenness (2006) is 0.5 for standardized TPI. This value corresponds approximately to 0.4 in normalized TPI for both case 1 and case2, hence objects with normalized TPI values over 0.4 are classified as upper position, and lower than -0.4 are classified as lower positions. 
Thematic Layers (Ridges and Streams): Ridges and Streams characterize the structure of the terrain and they are very closely associated with particular classes in the proposed classification scheme, namely; peak, ridge, and channel. They are included as thematic layer into classification independently, they are not necessarily considered within a level in the hierarchy of segmentation. Each thematic layer that is used for segmentation can lead to consistent access to its thematic information. Some information on thematic layers are transferred into objects; they are 'existence of any sections' that gives information about whether any object overlaps with stream or ridge lines, and 'number of overlapping sections' that gives information about how many sections of ridge lines fall into an object.

This information is of crucial importance as, objects that overlap with streams are considered to be channels. Objects that include 1 or 2 sections of ridge lines is considered to be a 'ridge' and objects that include 3 or more sections of ridge lines is considered to be a 'peak'. This rule set is adopted for use in the study from 'terrain topology' concept mentioned in section 2.2.3.2.2 (Terrain Network).

\subsubsection{Classification based on Geomorphometric Context}

Section 6.2.1 (Classification of Landforms based on local geometry) describe classification based on local geometry of the landscape to obtain landform classes based on semantic import of class descriptions into fuzzy rule sets that employ DTMs; slope and curvature. However these entities can be thought of as morphometric features rather than geomorphometric as they are particular to any surface (Wood, 1996). A terrain surface has a topology and it is composed of repetitive and nested systems (Milne et al., 1935). Landforms although not necessarily uniform, have specific organization, where for instance ridges are at the crests of hills and mountains; hence they are positioned at highest positions and/or correspond to the divides. Ridges in vertical plane are followed by shoulders and side slopes comes after. Foot slope is the lower part of a sloping terrain and it is adjacent to channels or plains. Slopes in the horizontal plane have a sequence of hollows and spurs according to whether they are concave or convex. Relative position across the landscape is incorporated to construct this organisation and add context into classification. Contextual information that reveals relative position of features across the landscape is gathered from a local elevation based model 'TPI'. Major streams and ridges that represent the terrain 
network are obtained out of surface flow model to provide guidance in allocating objects into specific landform classes like, 'ridge', 'peaks' and 'channels'.

For the proposed classification method, three sources of information is produced; 'Morphometric classes', 'terrain position classes' and 'terrain network features'. Three levels of hierarchy were generated as mentioned in section 6.1 (Segmentation). Segmentation level that carries information of morphometric classes is settled at the lowest level of the hierarchy (Level -1) and segmentation level that carries information of positional classes are settled at the highest level of the hierarchy (Level 1). Level 0 incorporates information both from 'Level -1 ' and 'Level 1'. These sub-level, super-level information is utilized in geomorphometric classification that is implemented at Level 0 (Figure 6.25). Terrain network is incorporated as independent thematic layers.

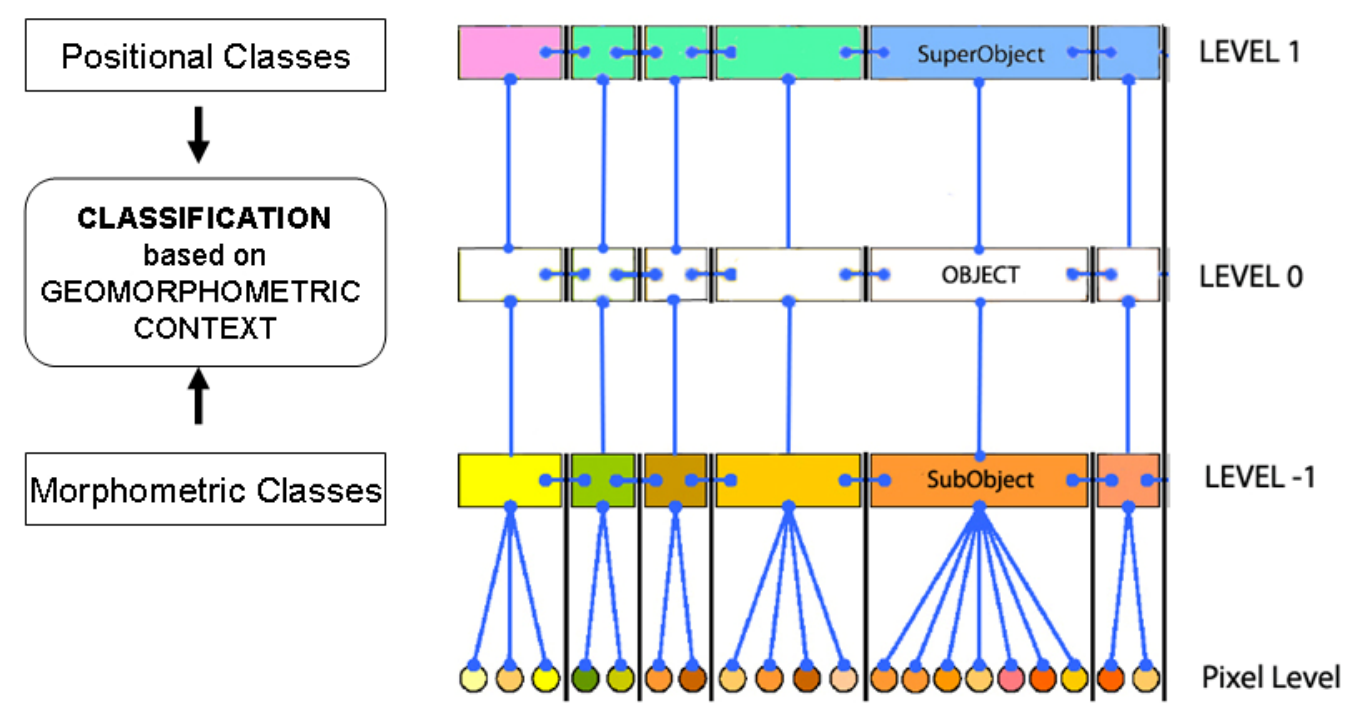

Figure 6.25: Hierarchical multi-level classification proposed for the classification.

Accordingly; landform 'peak' for instance is described with its form that comes from the morphometric classification based on local geometry; an object should be most similar to a 'peak' as described by membership functions. Actually at this stage feature is better called 'peak like feature' and one should understand that 'peak' here only pertains to form.

A peak is a surface specific feature that is characterized by intersecting ridge lines. If an object coincides with 3 or more sections of ridge line, than it should be referred to as 'peak' regarding terrain topology context mentioned in section 2.2.3.2.2 (Terrain Network). 
A peak is moreover local maxima that is characterized by relative terrain position. Therefore it should be located at the upper positions across the landscape.

To conclude; an object which either has a 'peak' form or which coincides with a ridge line intersection and that is at upper positions on the terrain is classified as 'peak' in geomorphometric classification. Figure 6.26 illustrates possible conditions for an object to be classified as 'peak'.

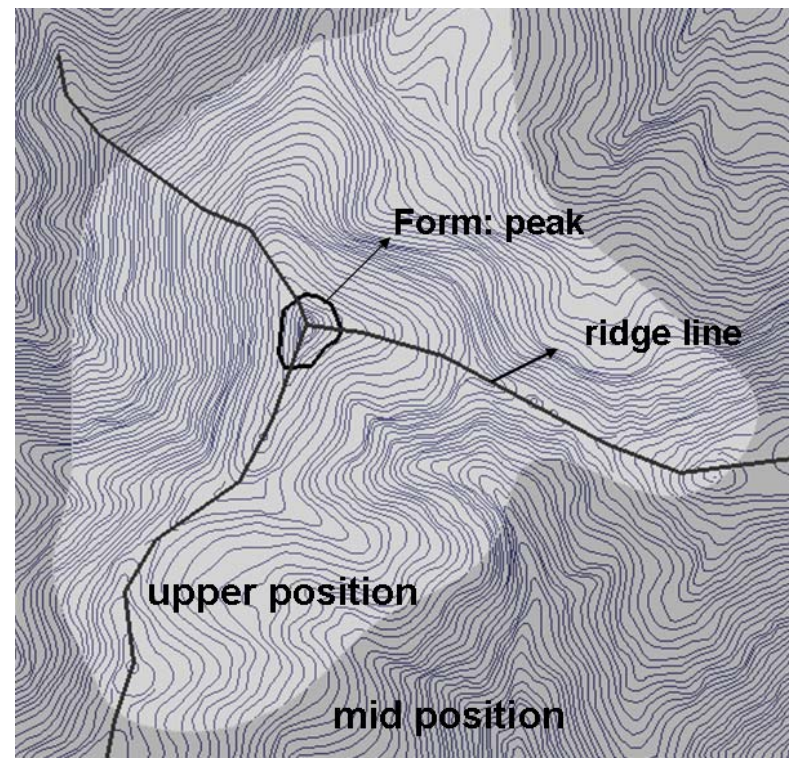

Figure 6.26: Conditions for an object to be classified as 'peak'.

Set of logical rules that utilize information from sub-level, super-level and thematic layers is incorporated for each of the 15 landforms as described in figures from (Figure 6.27 to Figure 6.41) with explanations.

Some of these rules are very simple where some of them are more complex. A ridge is for instance is associated with both terrain position, terrain network and other classes (Figure 6.28). However, 'plain' is only described with its form and it is not associated with geomorphometric context (Figure 6.30) as class 'plain' can be found at any position and can accompany any other class and it is not necessarily apart of a terrain network. 


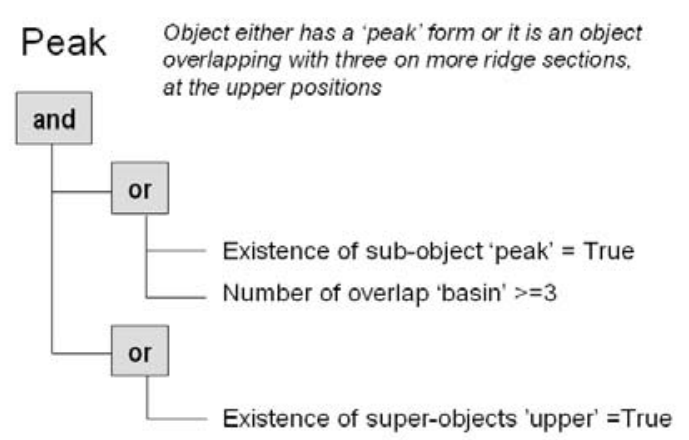

Figure 6.27: Logical rule set for geomorphometric classification of 'peak'

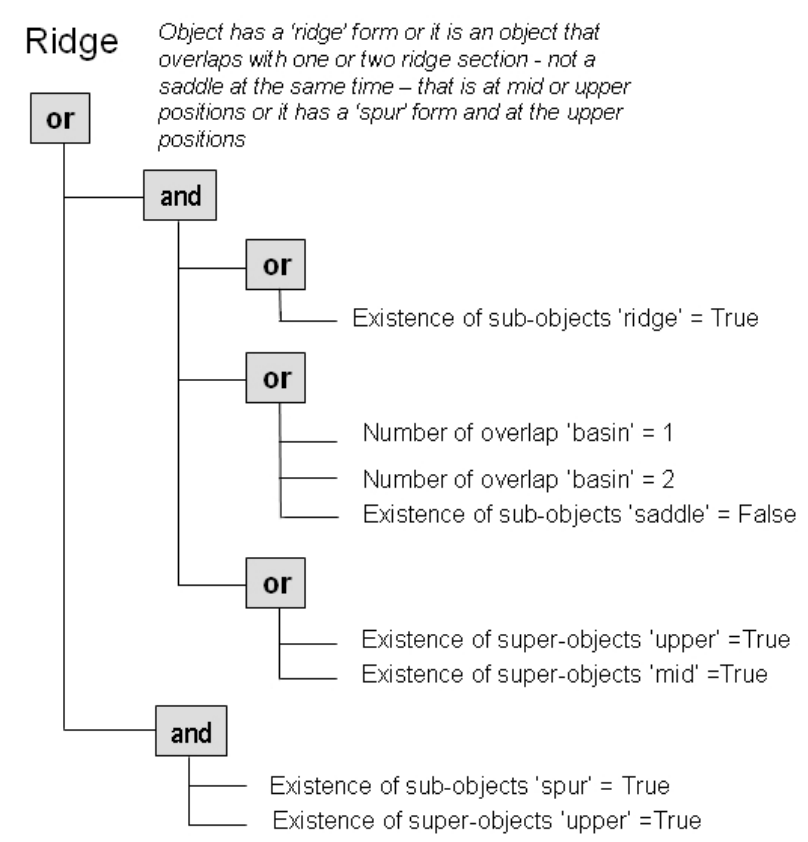

Figure 6.28: Logical rule set for geomorphometric classification of 'ridge'.

\section{Saddle Object either has a 'saddle' form and it is at the upper and mid positions at the upper positions}

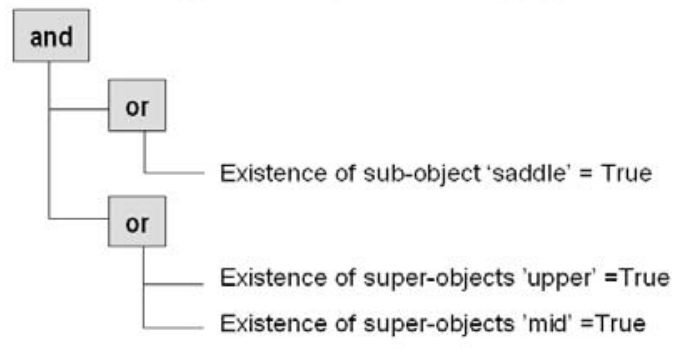

Figure 6.29: Logical rule set for geomorphometric classification of 'saddle' 


\section{Plain Object has a 'plain' form}

and

Existence of sub-object 'plain' $=$ True

Figure 6.30: Logical rule set for geomorphometric classification of 'plain'

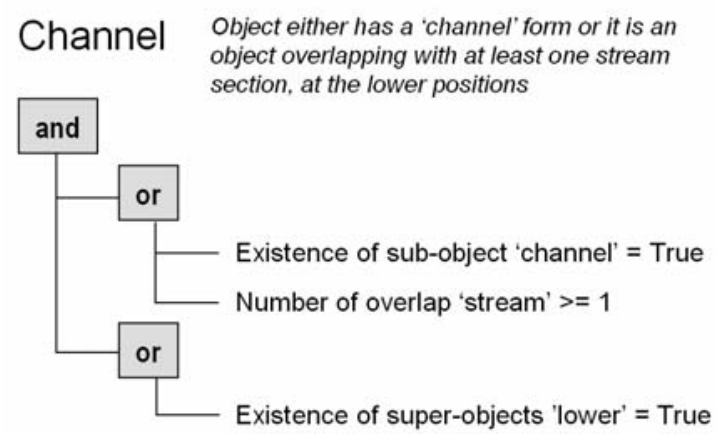

Figure 6.31: Logical rule set for geomorphometric classification of 'channel'

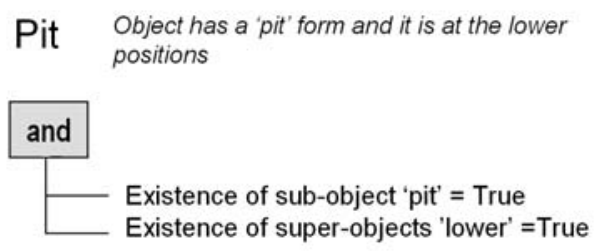

Figure 6.32: Logical rule set for geomorphometric classification of 'pit'

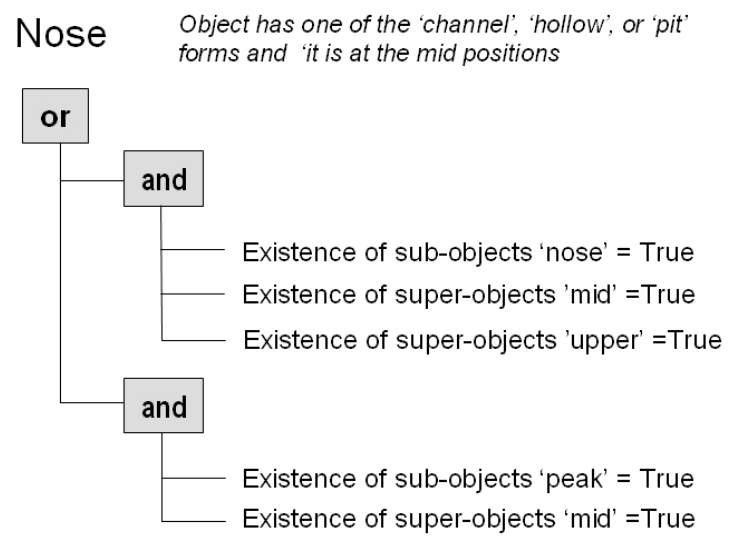

Figure 6.33: Logical rule set for geomorphometric classification of 'nose' 


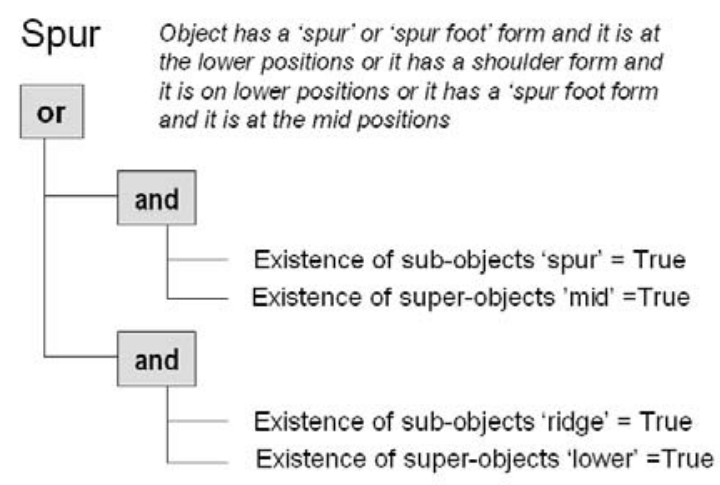

Figure 6.34: Logical rule set for geomorphometric classification of 'spur'

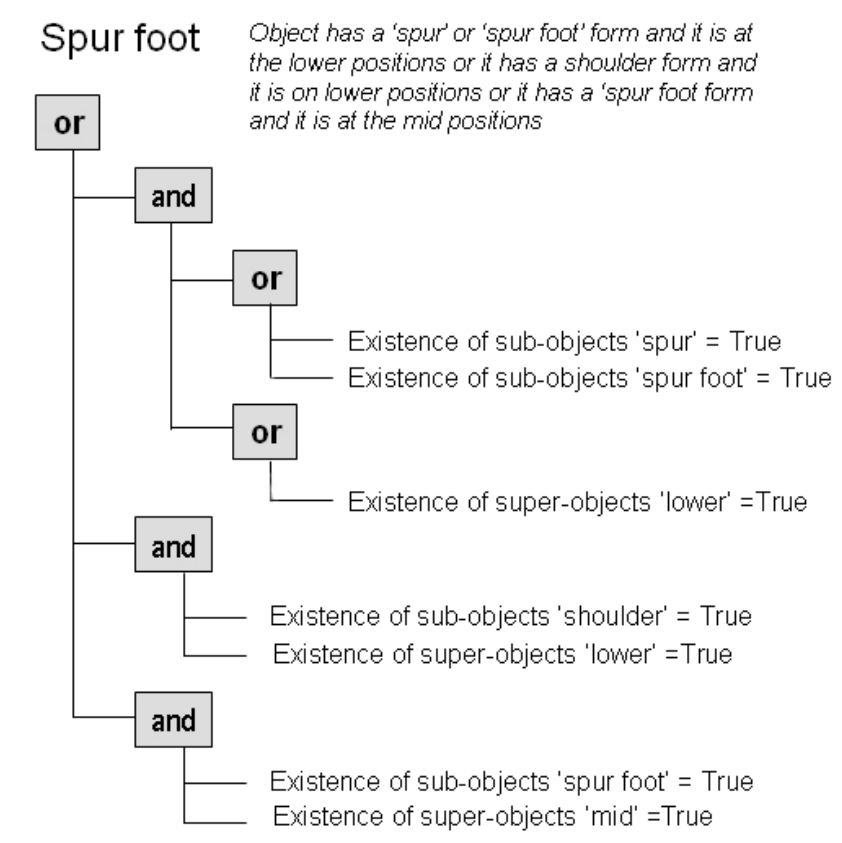

Figure 6.35: Logical rule set for geomorphometric classification of 'spur foot'.

\section{Shoulder Object has a 'shoulder' form and it is at mid or upper positions}

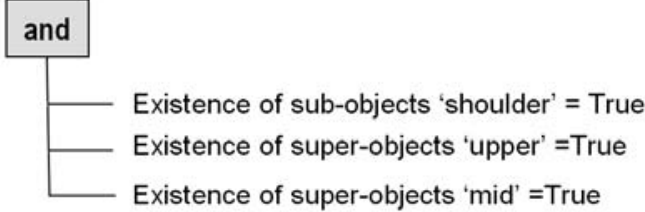

Figure 6.36: Logical rule set for geomorphometric classification of 'shoulder'. 


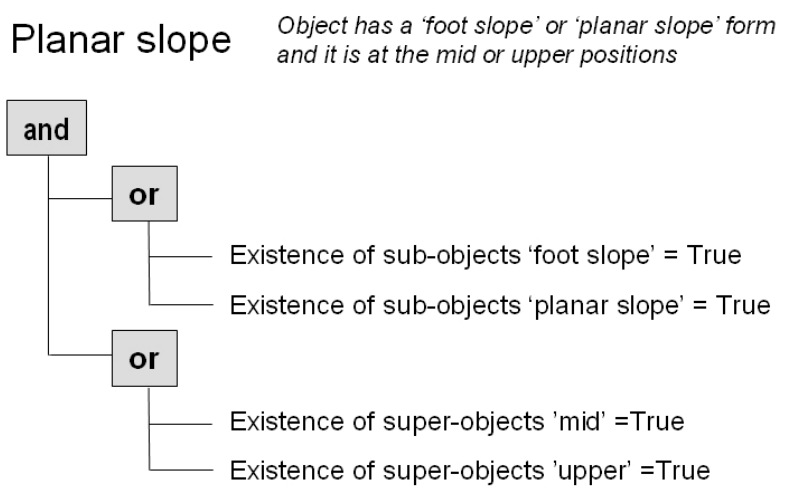

Figure 6.37: Logical rule set for geomorphometric classification of 'planar slope'.

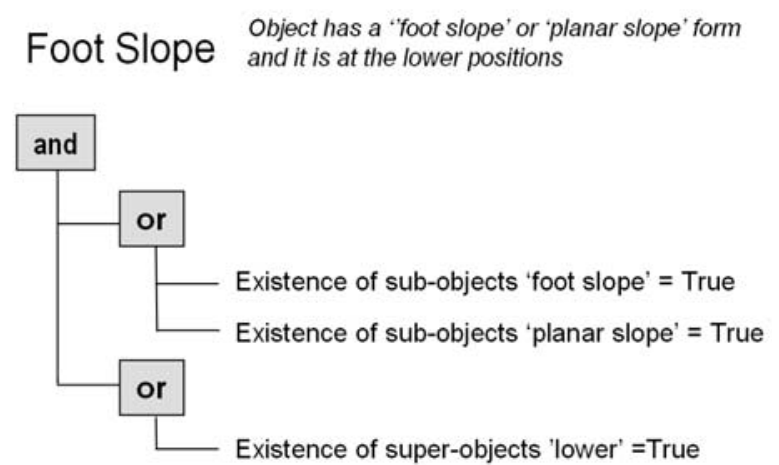

Figure 6.38: Logical rule set for geomorphometric classification of 'foot slope'.

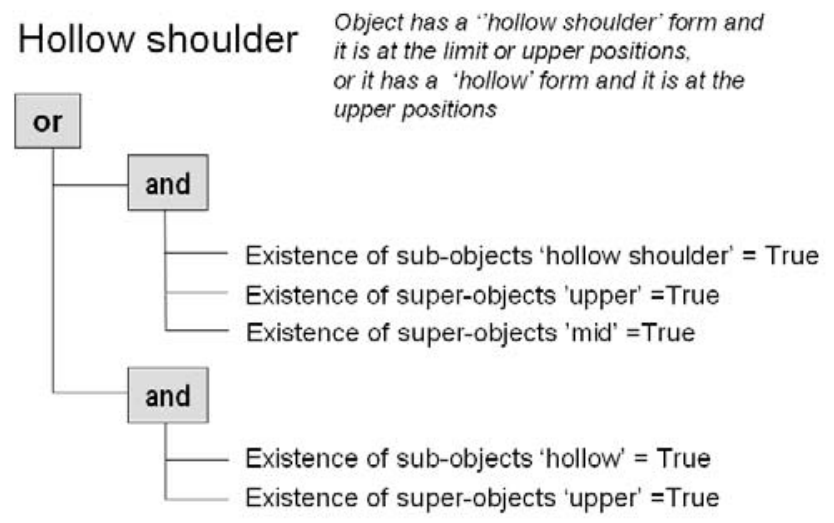

Figure 6.39: Logical rule set for geomorphometric classification of 'hollow shoulder'. 


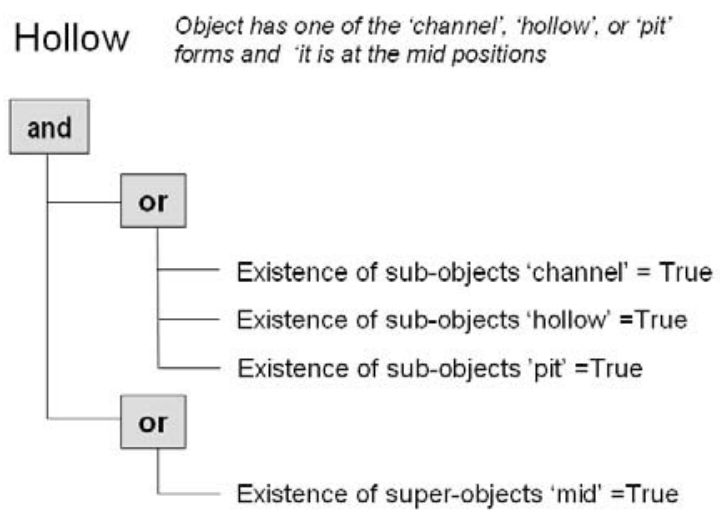

Figure 6.40: Logical rule set for geomorphometric classification of 'hollow'.

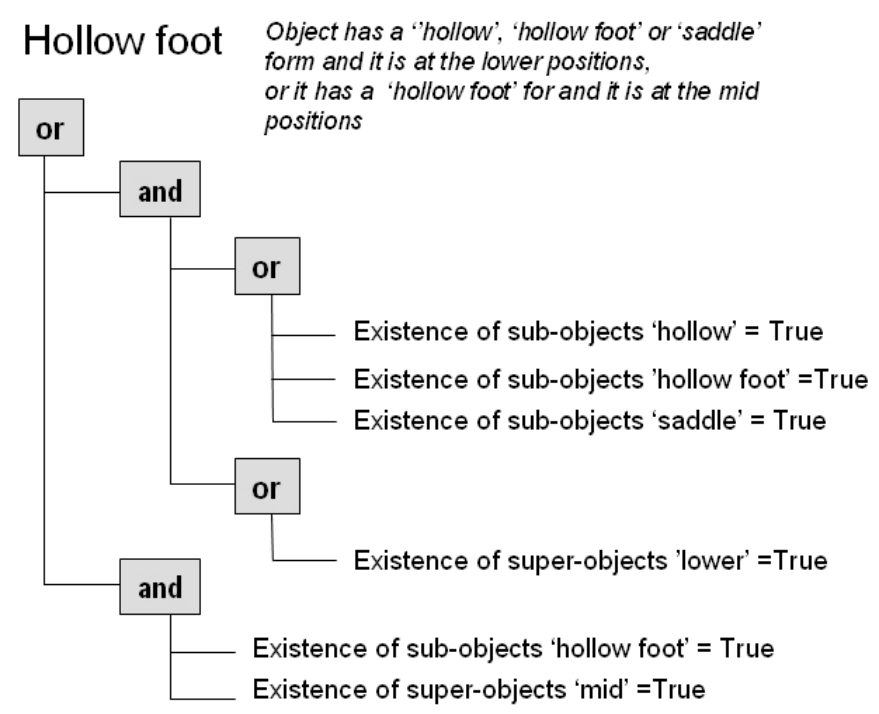

Figure 6.41: Logical rule set for geomorphometric classification of 'hollow foot'.

Geomorphometric classification which is applied through logical rules yield crisp classifications of landforms. Classification is applied on 'case1', 'case2', 'case1next' and 'case3'. Intention to apply classification on two case areas 'case1' and 'case2' is to see if the method works properly for two different areas. Visually it is observed that classification yield compatible results in both of the cases. There is no bias towards particular classes in one of the two cases.

Geomorphometric classification result for 'case1' is given in Figure 6.42. Classification result is also draped on 3D surface and viewed both from NW and SE directions (Figure $6.43)$. 


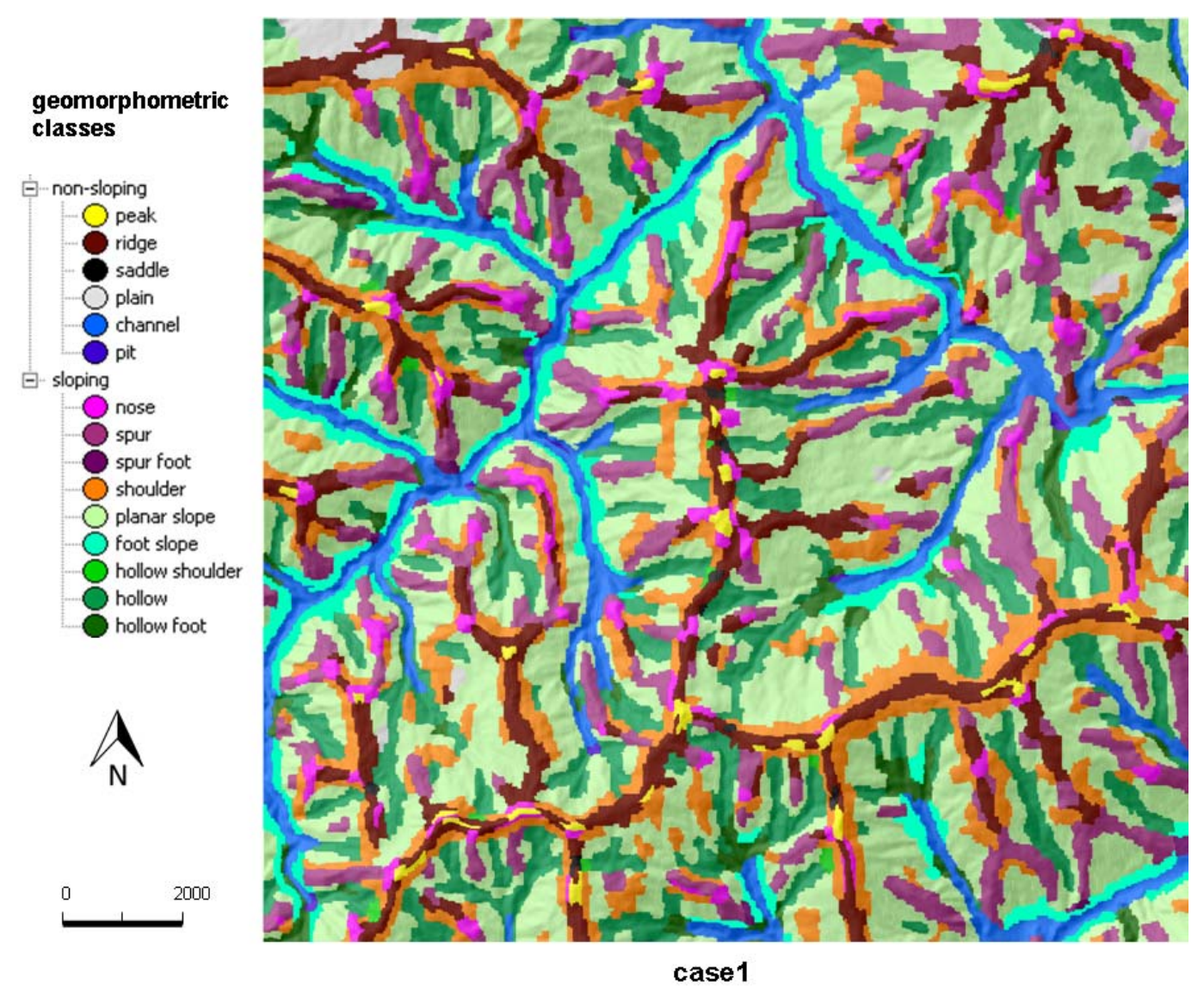

Figure 6.42: Geomorphometric classification result for 'case1'

Examining the out put visually, it appears that classes are well discriminated and the terrain nature can be easily recognised out of the resultant map. Channels and ridges are easily distinguished. Channels especially are very typical with their elongated and disconnected bodies. Foot slopes accompany channels. Hollows and spurs mainly show sequences across the horizontal plane and planar slopes dominate the whole case. Peaks are observed at their proper locations usually where ridges connect.. Ridges are continuous but ca be disconnected with peaks and a few saddles.

Result for 'case2' is given in Figure 6.44. Classification result is also draped on 3D surface and viewed from NW and SE directions (Figure 6.45). 


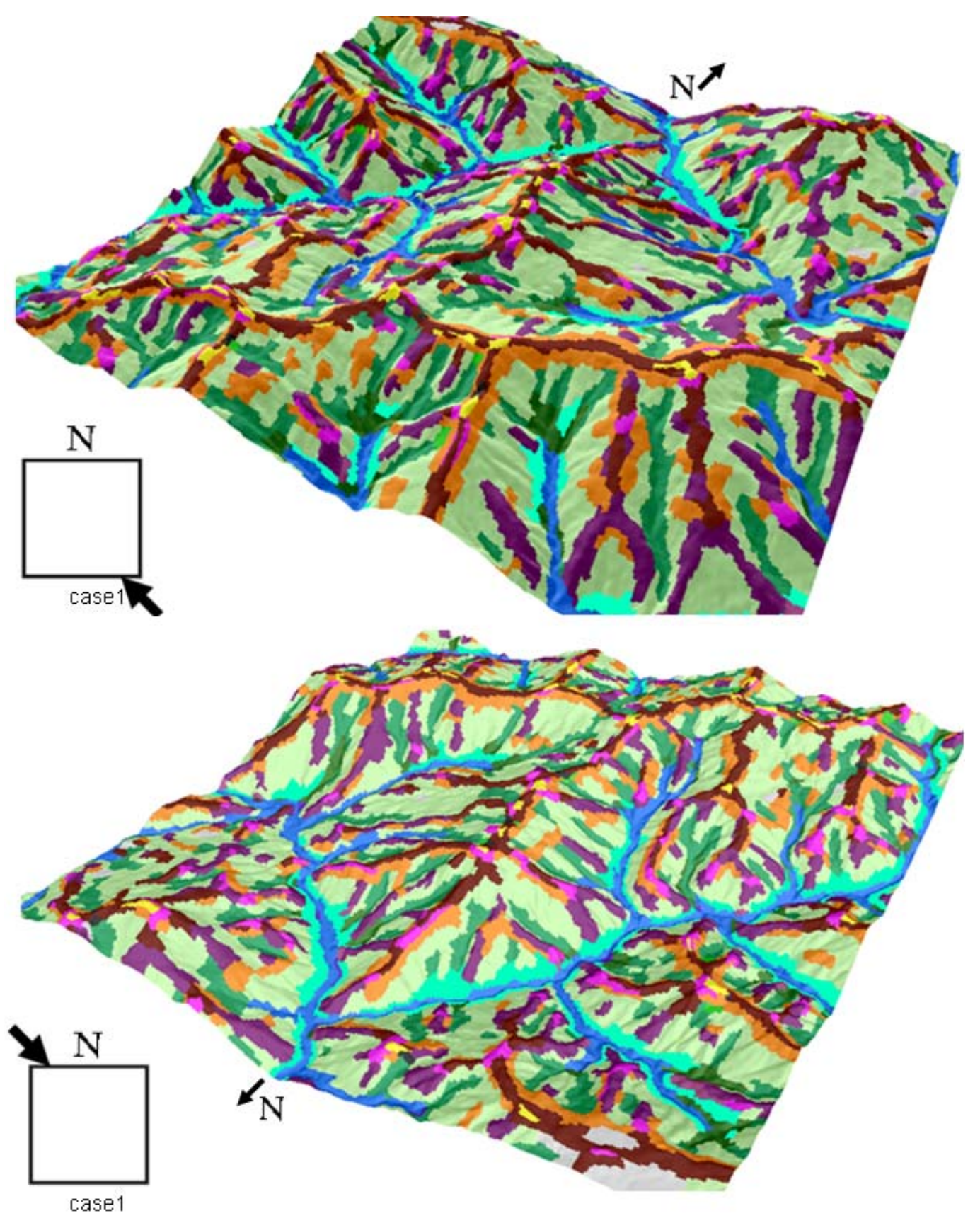

Figure 6.43: 3D views of Geomorphometric classification result for 'case 1' 


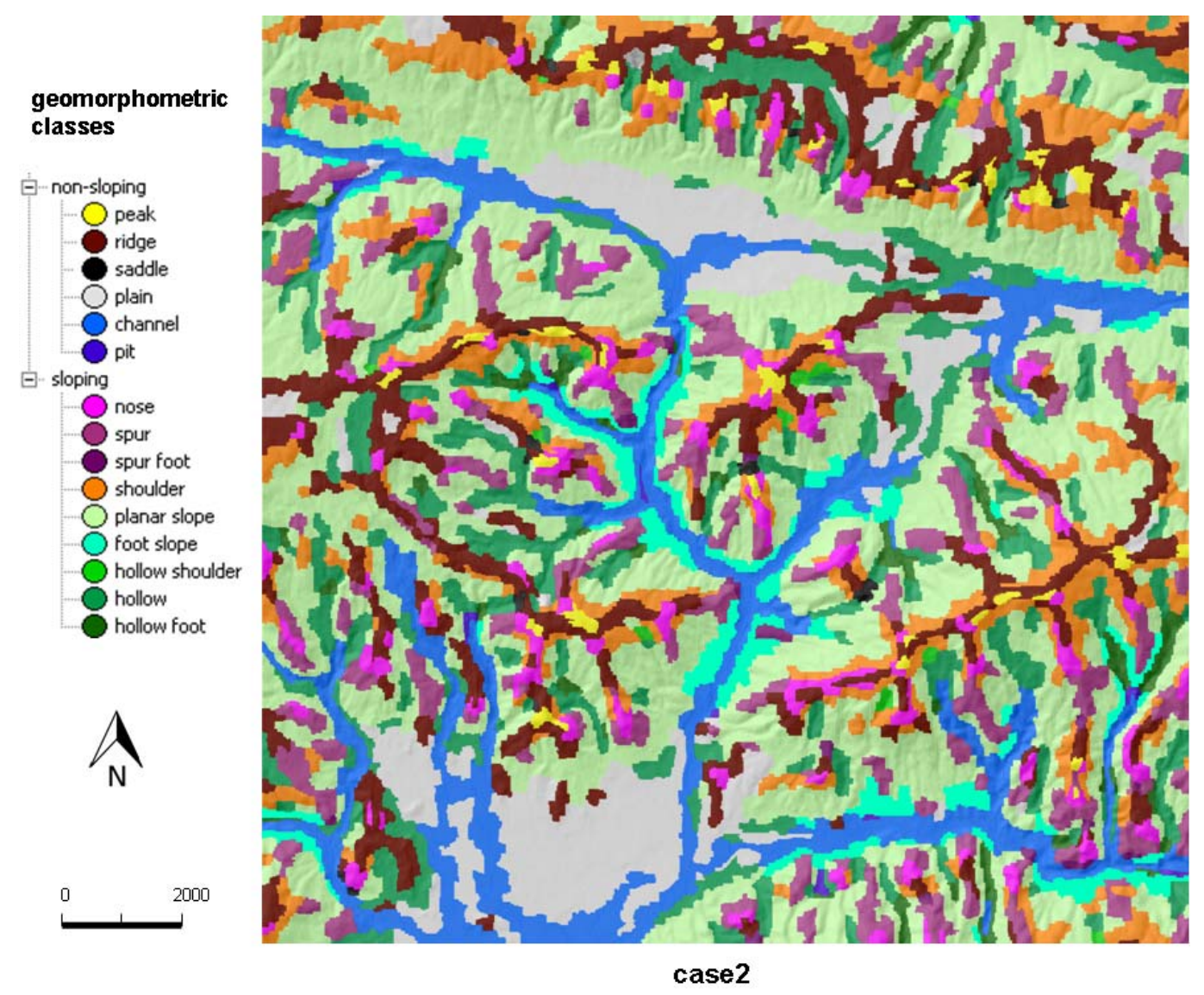

Figure 6.44: Geomorphometric classification result for 'case2'

Geomorphometric classification of case2 appears to represent typical nature of the landscape. This area is smoother dominated by some hills and there are more flat areas and they are classified as 'plain' in 'case2'. Different from 'case1', more area is allocated to class 'plain'. Channels are continuous but tend to depict irregularities due to flatness of the topography. Channels here are accompanied with plains rather than foot slopes different from case1. Channels are also wider as this landscape is less dissected due to being at an older geomorphologic stage compared to case1 as mentioned in Chapter 4 (Study Area). Landscape also appears to depict more roughness comparing the classification results form 'case1'. 


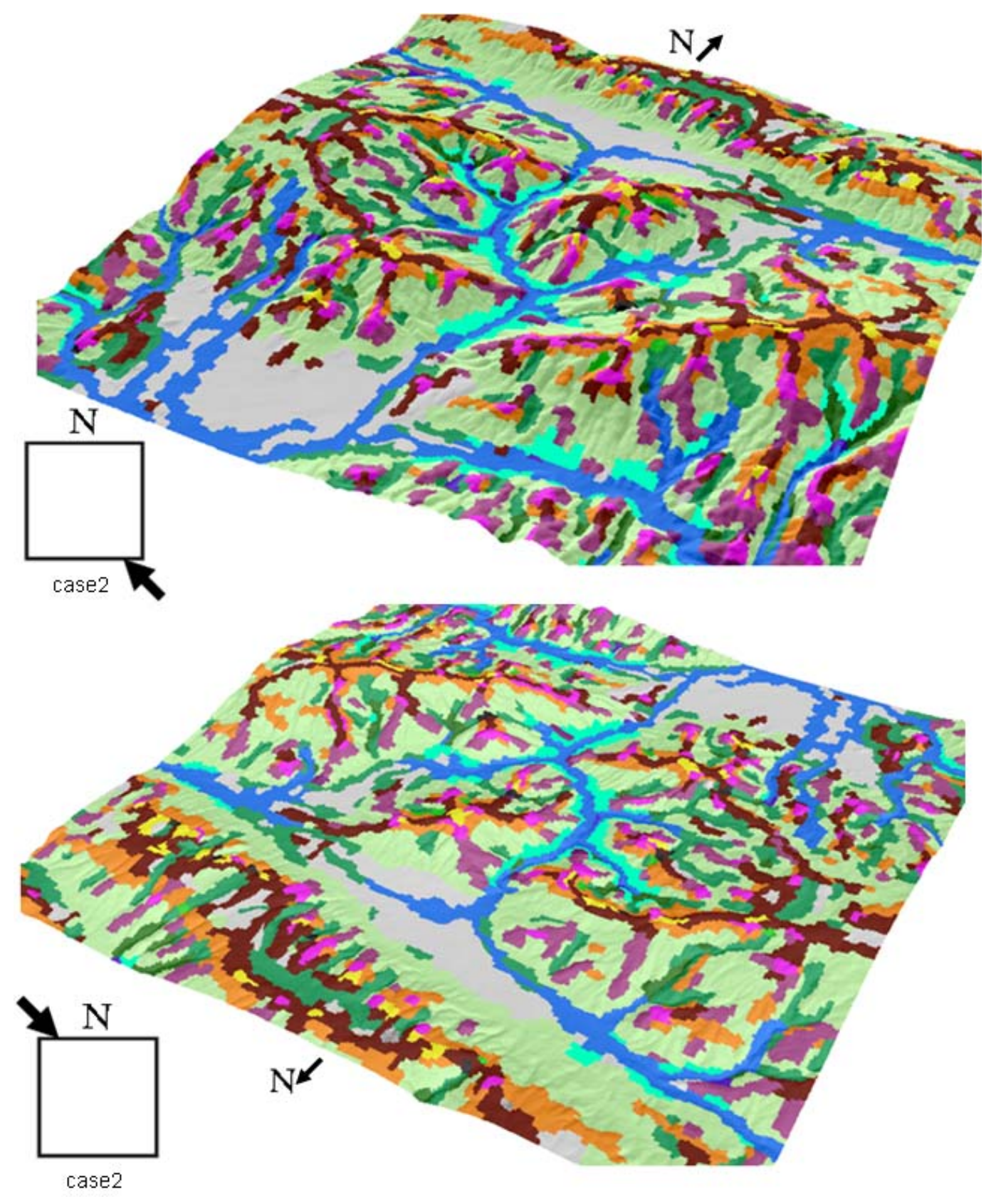

Figure 6.45: 3D views of Geomorphometric classification result for 'case2' 
Finally, overlapping area of 'case1' and 'case1next' is provided (Figure 6.46). Intention to apply the method to two overlapping areas is to see degree to which this overlapping area is similar in each of the classifications. Results at the first glance appear to be consistent however some discrepancies are observed when examined. Classes are usually consistent but their sizes and borders of extents seem to change slightly.
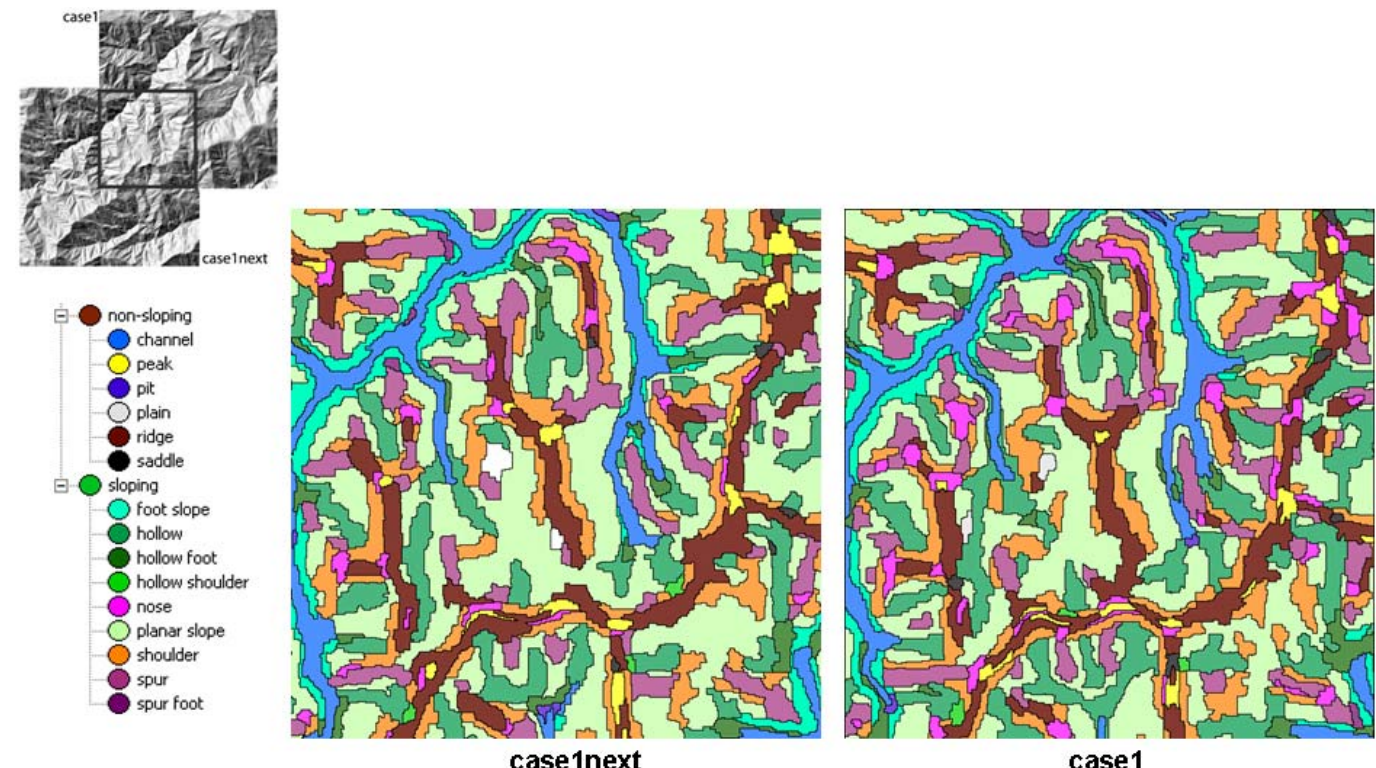

Figure 6.46: Overlapping area classified as a part of morphometric classification of 'caselnext' (left) and 'case1' (right) 


\section{CHAPTER 7}

\section{RESULTS AND DISCUSSIONS}

This chapter is allocated for the evaluation and discussion of the method in terms of the outcomes of results and the method itself. Accordingly, chapter has been subdivided into two parts as 'Evaluation of the results' and 'Discussion of the method' where in the first part classification results are evaluated as outcomes of this research and in the second part, method is generally being discussed based on classes, scale, parameters, case areas, data preparation, analysis methods, assumptions, and possible alternatives.

\subsection{Evaluation of the results}

Evaluation of the results is crucial part of the work in image classification. Classification results should be tested for their quality and the results should be reported. There are standard accuracy assessment procedures used in evaluating results originating from classification of satellite imagery particularly for extracting land use and land cover. Accordingly, classification results for natural or man-made features are compared with another data set that is regarded as ground truth or observations on the ground. Rate of match between the observations and the results depict the accuracy of the classification. However, landforms are quite different in characteristics compared to land use or land cover classes. These can be mentioned as;

(i) landforms are different compared to features that are 'real' or of 'natural kinds' as mentioned in section 2.2.2 (Landform ontology). Landforms of general geomorphometry are rather described based on semantics,

(ii) terrain has a continuous nature and landforms has indeterminate boundaries both in attribute and spatial space, which can be handled via fuzzy representations

(iii) landform classification is strongly related with the window of perception, hence is scale-dependent. 
Therefore; accuracy assessment for landform classification is problematic. There is lack of quantitative methods for assessing the accuracy of DTM-based classifications (Dragut and Blaschke, 2009) and existing ones can be barely applicable to landform classification. Regarding particular nature of landforms, their classification constitutes a special case in image classification. Therefore, a term like for instance accuracy assessment' is avoided and rather 'evaluation of the results' is considered to be more proper.

Proposed classification method is implemented at two parts; 'morphometric' and 'geomorphometric'. Different case areas are studied and different sets of data with different scales are employed. This was intentionally to examine the method from different perspectives. Therefore evaluations are made regarding some particular intentions.

Evaluation of scale effect: Scale effect on morphometric classification is examined; case1fine, case 1 and case1coarse datasets were classified based on local geometry and the differences were examined.

Evaluation of classification stability and efficiency: Stability of fuzzy morphometric classification and efficiency of rules in classifying morphometric landforms is evaluated.

Evaluation of classification based on Geomorphometric context: Morphometric and geomorphometric classifications are compared to understand whether geomorphometric context has added to landform classification.

Evaluation of classification on a flat topography: Geomorphometric classification is performed on a very flat topography that is dominated by flat plains to test the efficiency of method in an area that portrays only a few of the proposed classes.

Evaluation of Classification Consistency: Consistency of geomorphometric classification on two overlapping areas is compared to check if the method produces consistent results.

External evaluation: Geomorphometric classification results were compared for a set of random locations with the suggestions of several geographers and geomorphologists who are experienced in landform classification. 


\subsubsection{Scale effect}

Morphometric classification is applied on three datasets which belong to the same extents of area however, with DTMs that are derived at different scales utilizing different window sizes. Percentage of areas in each scale that were assigned to each morphometric classes are shown in Table 7.1. Highlighted values indicate the scale at which a morphometric class is significantly higher compared to classifications at the other scales.

Table 7.1: The percentage of areas of objects at three scales of case1; 'caselfine', 'casel', 'case1 coarse' respectively

\begin{tabular}{lccc}
\hline & $\begin{array}{c}\text { case1 fine } \\
(\text { area \%) }\end{array}$ & $\begin{array}{c}\text { case1 } \\
(\text { area } \%)\end{array}$ & $\begin{array}{c}\text { case1 coarse } \\
(\text { area \%) }\end{array}$ \\
\hline peak & 0.1 & 0.7 & 1.3 \\
ridge & 4.9 & 4.5 & 4.8 \\
saddle & 0.0 & 0.2 & 0.4 \\
plain & 2.5 & 1.0 & 0.4 \\
channel & 4.3 & 4.7 & 5.8 \\
pit & 0.0 & 0.6 & 1.2 \\
\hline nose & 2.0 & 3.0 & 2.1 \\
spur & 15.0 & 16.1 & 15.8 \\
spur foot & 0.0 & 0.1 & 0.2 \\
shoulder & 7.4 & 11.9 & 13.5 \\
planar slope & 43.4 & 30.0 & 29.8 \\
foot slope & 6.1 & 10.3 & 12.2 \\
hollow shoulder & 0.0 & 0.1 & 0.1 \\
hollow & 13.6 & 16.1 & 11.9 \\
hollow foot & 0.7 & 0.6 & 0.4 \\
\hline
\end{tabular}

Figure 7.1 that is plotted out of Table 7.1 portrays relative portion of areas per class in each of the three classification results. Areal extents of the classes tend to shift slightly especially for non-sloping forms, however there is more divergence in sloping forms, especially area of 'planar slope' in 'case1fine' increases considerably. Prominence of planar slope is also very apparent in the classification map (Figure 6.21). This might be due to deep curved nature of terrain which can be detected at coarser scale. As the hollows and spurs enlarge, planar slope is consumed by wider spurs and hollows. At a finer scale most of these curved features become planar patches as the window of perception is relatively small. Class 'plain' is similarly higher in 'caselfine' compared to other scales. Peaks and shoulders enlarge in 'case1 coarse' as the classification tends to capture wide convexities. In 'case1' there is more uniformity of distribution compared to other scales. This might be an indication that not specific classes but majority of the classes characterizing the terrain can be captured at this scale. 
Plot of areas in Figure7.1 although gives some information on total areal distribution of classes, it does not give an idea about their spatial distribution. Therefore, for three cases; 'case1 fine', 'case1' and 'case1 coarse', classification results that are same for all of the three classifications are extracted out of the resultant maps. Although there are not too many overlapping classes, ridges and channels and other features like spur, hollow could be recognizably observed (Figure 7.2).

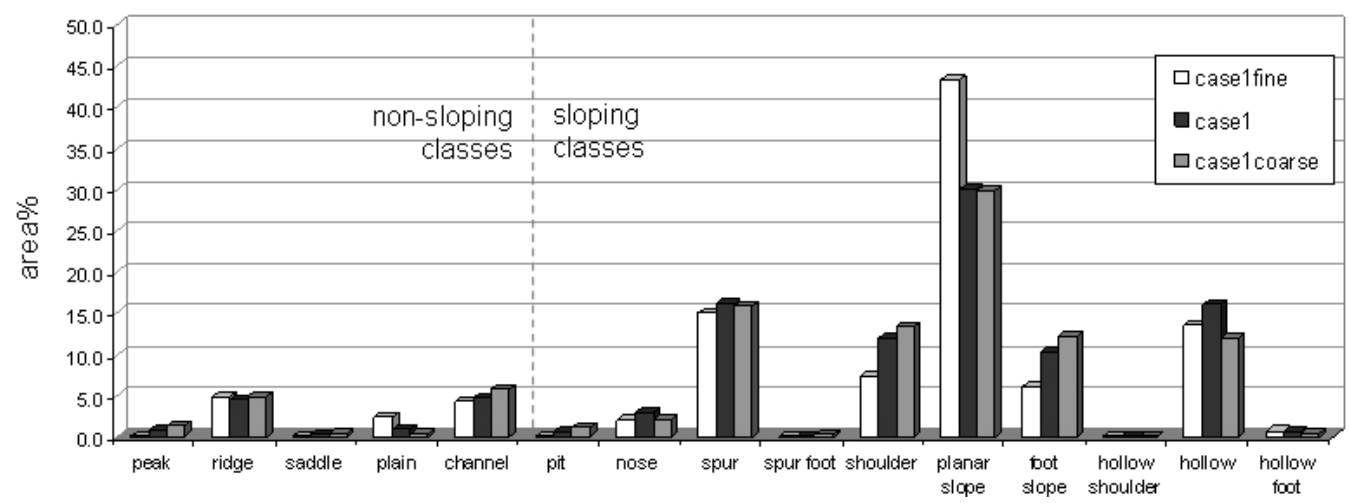

Figure 7.1 Total area of per class of morphometric classification for 'case1fine', 'case1' and 'caselcoarse'. First six of the classes pertain to non-sloping and other nine classes pertain to morphometric classes
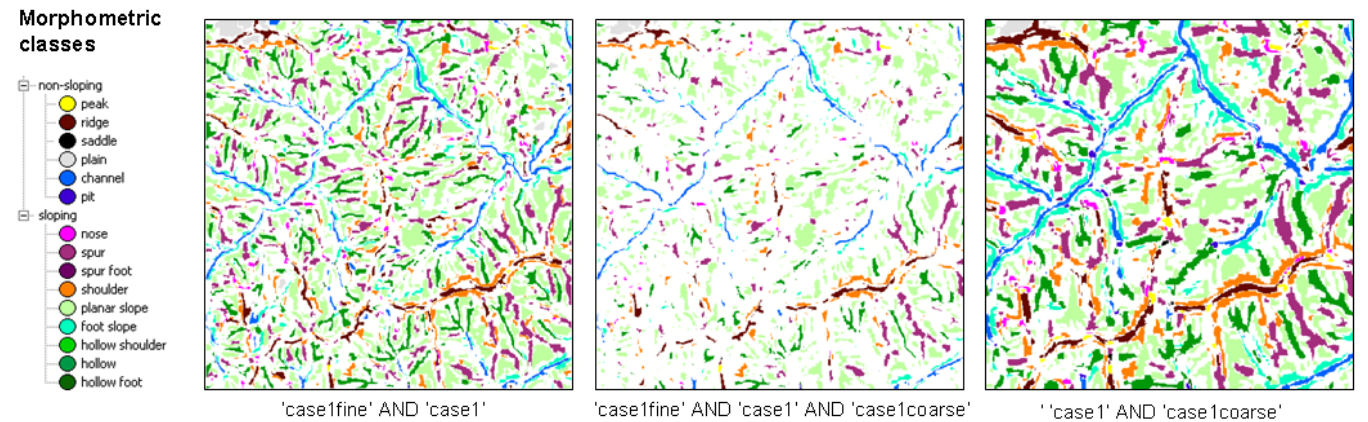

Figure 7.2: Spatial distribution of the intersecting morphometric classes in 'case1' and 'casel fine' (left), all cases (middle), 'case1' and 'caselcoarse'(right).

Classification produces different classes for the same locations as the dataset is prepared to represent particular scales. Those classes are thought of as scale-dependent. However, it is intended in this evaluation to observe scale-independency if any. Therefore a "change matrix' is utilized to make pairwise comparisons between finer to coarser scales for 'case1'. Percent of change from 'caselfine' to 'case1' is illustrated in Figure 7.3. It can bee understood from the plot (Figure 7.3) that most significant change is in planar slope as it has 
drastically decreased in extents in case 1 compared to case1fine, on the other side shoulders, hollows, foot slopes, spurs which are also sloping elements have increased. This may indicate that significant curvedness of terrain is being detected at 'case1' scale, so that curved features increase and planar slopes are consumed by them.

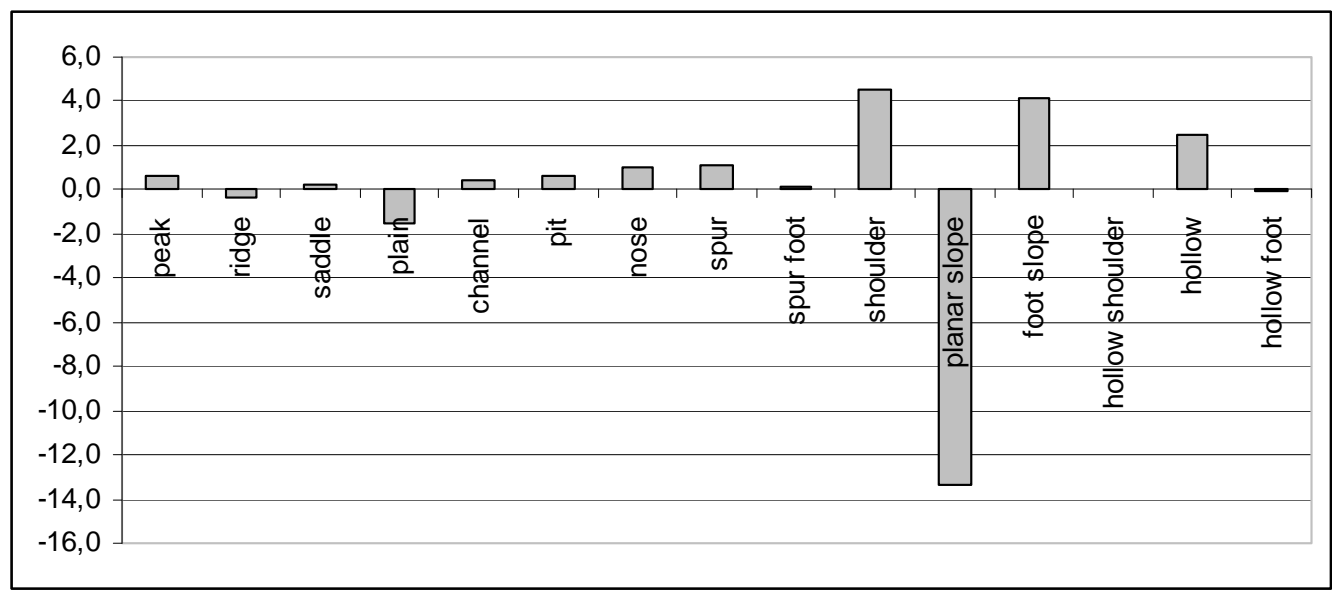

Figure 7.3 Percent of difference from fine to coarse resolution: casel fine to case coarse

Similarly percentage of change from 'case1' to 'case1coarse' is illustrated in Figure 7.4. Most significant decrease is in hollow, foot slope and shoulders and channels have increased instead. Examining tables it can be seen that peak, channel, shoulder and foot slope tend to increase in coarser scales.

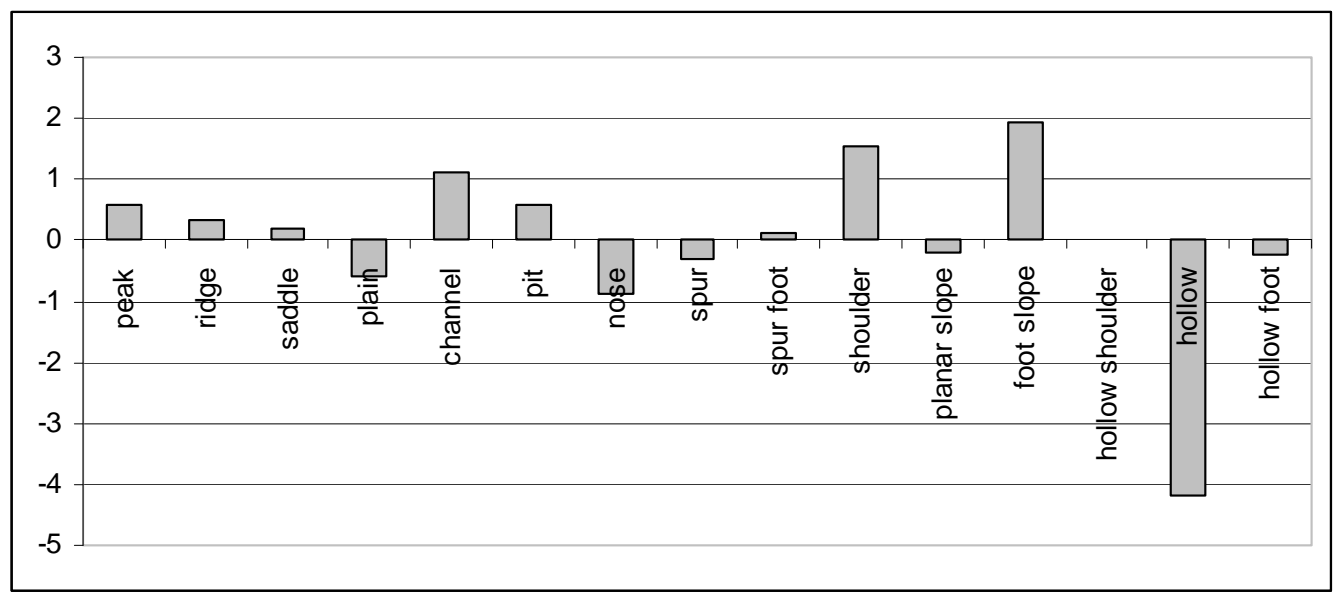

Figure 7.4: Percent of difference from fine to coarse resolution: case1 to case coarse 
It is very obvious that window of perception (scale) has effects on the outcomes of the classification of landforms. In a classification attempt to obtain same set of classes, a coarser scale is likely to produce more generalized classes. Especially on landscapes with significant changes in form. Curved forms like, peak, ridge, shoulders, hollows and spurs tend to get enlarged and consume areas occupied by planar forms that are produced in finer scale classifications. Pairwise evaluations on the morphometric classifications show that more than half of the total area is classified differently when comparison is made between different scales. More over it is observed that some of the classes are preserved in all of the classifications. Those classes are considered to have relatively more scale independency compared to other classes. For instance; ridges are observed to be most resistant classification over scales as it is preserved with relatively higher areal extent in all of the three scales of classification.

\subsubsection{Classification stability and efficiency}

There are some techniques to evaluate the classification stability and efficiency of rule sets developed for fuzzy classification. 'classification stability' and 'best classification result' techniques were employed to test the classification model success for the study. However these techniques are only applicable to fuzzy classes and therefore evaluation covers morphometric classes only.

'Classification stability' evaluates the differences in degrees of membership between the best classification result and the second best classification result for each object. The smaller the value, more ambiguous the classification is. 'Best classification result' on the other hand assesses how high is the memberships of belonging to a class, hence; how good the objects of a class satisfy the class description.

'Classification stability' and 'best classification result' techniques were performed on the morphometric classification results and the results are provided in Tables 7.2 to 7.4 for case 1 fine, case 1 and case 1 coarse respectively.

Classification stability and best classification results scores for evaluated cases tend to reflect similarities for classes; i.e. they pose low values or high values together for particular classes. It is not observed that a class with a high stability poses low best classification 
value. Therefore, these two measures are not plotted together but separately for three scales of classification.

Table 7.2: Classification stability and best classification results for morphometric classification of 'case1 fine' sorted in descending order

\begin{tabular}{|l|r|r|}
\hline \multicolumn{2}{|l|}{ Classification Stability } & \\
\hline Class & \multicolumn{1}{l|}{ Mean } & \multicolumn{1}{l|}{ StdDev } \\
\hline planar slope & 0.163278 & 0.08703 \\
\hline foot slope & 0.135618 & 0.084714 \\
\hline channel & 0.116366 & 0.090136 \\
\hline ridge & 0.107416 & 0.078938 \\
\hline shoulder & 0.102482 & 0.061973 \\
\hline hollow & 0.1008 & 0.059858 \\
\hline spur & 0.093491 & 0.053203 \\
\hline plain & 0.089506 & 0.06463 \\
\hline peak & 0.076521 & 0.054189 \\
\hline hollow shoulder & 0.072754 & 0.031004 \\
\hline saddle & 0.068893 & 0.04658 \\
\hline nose & 0.067394 & 0.045191 \\
\hline hollow foot & 0.059711 & 0.043774 \\
\hline spur foot & 0.04456 & 0.033432 \\
\hline pit & 0.024086 & 0.026692 \\
\hline
\end{tabular}

\begin{tabular}{|l|r|r|}
\hline \multicolumn{2}{|l|}{ Best Classification } & \\
\hline Class & Mean & StdDev \\
\hline planar slope & 0.801453 & 0.096493 \\
\hline channel & 0.759198 & 0.112659 \\
\hline foot slope & 0.72242 & 0.09018 \\
\hline ridge & 0.709483 & 0.099719 \\
\hline peak & 0.707313 & 0.081017 \\
\hline plain & 0.701385 & 0.093608 \\
\hline hollow & 0.698715 & 0.070407 \\
\hline spur & 0.694762 & 0.073712 \\
\hline shoulder & 0.685619 & 0.079587 \\
\hline pit & 0.676863 & 0.086368 \\
\hline nose & 0.654359 & 0.077006 \\
\hline saddle & 0.644185 & 0.092533 \\
\hline hollow foot & 0.642706 & 0.062368 \\
\hline spur foot & 0.592496 & 0.043912 \\
\hline hollow shoulder & 0.568733 & 0.050141 \\
\hline
\end{tabular}

Figure 7.5 and Figure 7.6 illustrates classification stability and best classification result of three scales of morphometric classification: 'case1fine', 'case1' and 'case1coarse' respectively. First six of the classes on the $\mathrm{x}$ axis represent non-slopping morphometry and the remaining nine classes represent sloping morphometry. What is common to all classifications is, classes; 'saddle', 'pit', 'spur foot', 'hollow shoulder' and 'hollow foot' pose low classification stability and they are not in good fit with class descriptions compared to other classes regarding their low best classification results. Ridge, channel, planar slope and foot slope are more stable and they have better fit with class descriptions. It is also observed that sloping elements: classes that can be thought of as components of a wider class such as nose, spur and spur foot (broadly can be categorized as spurs) pose low stability and efficiency values for nose and spur foot. Similarly hollow shoulder, hollow and hollow foot (broadly can be categorized as hollows) pose low stability and efficiency values for hollow shoulder and hollow foot. 
Table 7.3: Classification stability and best classification results for morphometric classification of 'case1' sorted in descending order

\begin{tabular}{|l|r|r|}
\hline \multicolumn{3}{|l|}{ Classification Stability } \\
\hline Class & \multicolumn{1}{l|}{ Mean } & \multicolumn{1}{l|}{ StdDev } \\
\hline planar slope & 0.144923 & 0.025102 \\
\hline channel & 0.137542 & 0.043051 \\
\hline foot slope & 0.116629 & 0.035152 \\
\hline ridge & 0.114985 & 0.043736 \\
\hline plain & 0.100139 & 0.020192 \\
\hline shoulder & 0.092437 & 0.051102 \\
\hline spur & 0.091898 & 0.059752 \\
\hline hollow & 0.091103 & 0.0549 \\
\hline peak & 0.075648 & 0.054028 \\
\hline pit & 0.072772 & 0.059692 \\
\hline spur foot & 0.071403 & 0.06743 \\
\hline nose & 0.065691 & 0.081923 \\
\hline saddle & 0.058182 & 0.076415 \\
\hline hollow foot & 0.047808 & 0.086117 \\
\hline hollow shoulder & 0.04009 & 0.087924 \\
\hline
\end{tabular}

\begin{tabular}{|l|l|r|}
\hline \multicolumn{2}{|l|}{ Best Classification } & \\
\hline Class & Mean & StdDev \\
\hline planar slope & 0.801453 & 0.096493 \\
\hline channel & 0.759198 & 0.112659 \\
\hline foot slope & 0.72242 & 0.09018 \\
\hline ridge & 0.709483 & 0.099719 \\
\hline peak & 0.707313 & 0.081017 \\
\hline plain & 0.701385 & 0.093608 \\
\hline hollow & 0.698715 & 0.070407 \\
\hline spur & 0.694762 & 0.073712 \\
\hline shoulder & 0.685619 & 0.079587 \\
\hline pit & 0.676863 & 0.086368 \\
\hline nose & 0.654359 & 0.077006 \\
\hline saddle & 0.644185 & 0.092533 \\
\hline hollow foot & 0.642706 & 0.062368 \\
\hline spur foot & 0.592496 & 0.043912 \\
\hline hollow shoulder & 0.568733 & 0.050141 \\
\hline
\end{tabular}

Table 7.4: Classification stability and best classification results for morphometric classification of 'casel coarse' sorted in descending order

\begin{tabular}{|l|r|r|}
\hline \multicolumn{3}{|l|}{ Classification Stability } \\
\hline Class & \multicolumn{1}{l|}{ Mean } & \multicolumn{1}{l|}{ Std.Dev } \\
\hline planar slope & 0.141731 & 0.084123 \\
\hline channel & 0.137185 & 0.083269 \\
\hline foot slope & 0.132877 & 0.076119 \\
\hline ridge & 0.128468 & 0.089087 \\
\hline plain & 0.122165 & 0.056895 \\
\hline pit & 0.097468 & 0.054172 \\
\hline shoulder & 0.096196 & 0.062135 \\
\hline peak & 0.095845 & 0.080123 \\
\hline spur & 0.089339 & 0.053831 \\
\hline hollow & 0.082313 & 0.04731 \\
\hline saddle & 0.062151 & 0.046093 \\
\hline nose & 0.047395 & 0.039172 \\
\hline hollow shoulder & 0.03441 & 0.019627 \\
\hline hollow foot & 0.03162 & 0.019092 \\
\hline spur foot & 0.027138 & 0.021091 \\
\hline
\end{tabular}

\begin{tabular}{|l|r|r|}
\hline \multicolumn{2}{|l|}{ Best Classification } & \\
\hline Class & \multicolumn{1}{l|}{ Mean } & \multicolumn{1}{l|}{ Std.Dev } \\
\hline planar slope & 0.79872 & 0.09022 \\
\hline channel & 0.767674 & 0.101604 \\
\hline plain & 0.747127 & 0.037381 \\
\hline ridge & 0.741354 & 0.109576 \\
\hline foot slope & 0.738189 & 0.087218 \\
\hline peak & 0.72601 & 0.099425 \\
\hline shoulder & 0.698771 & 0.078872 \\
\hline spur & 0.694156 & 0.072951 \\
\hline pit & 0.683608 & 0.076244 \\
\hline hollow & 0.683407 & 0.074529 \\
\hline saddle & 0.672118 & 0.07144 \\
\hline nose & 0.642973 & 0.070757 \\
\hline hollow foot & 0.606627 & 0.034189 \\
\hline spur foot & 0.590642 & 0.075449 \\
\hline hollow shoulder & 0.574293 & 0.033989 \\
\hline
\end{tabular}


classification stability

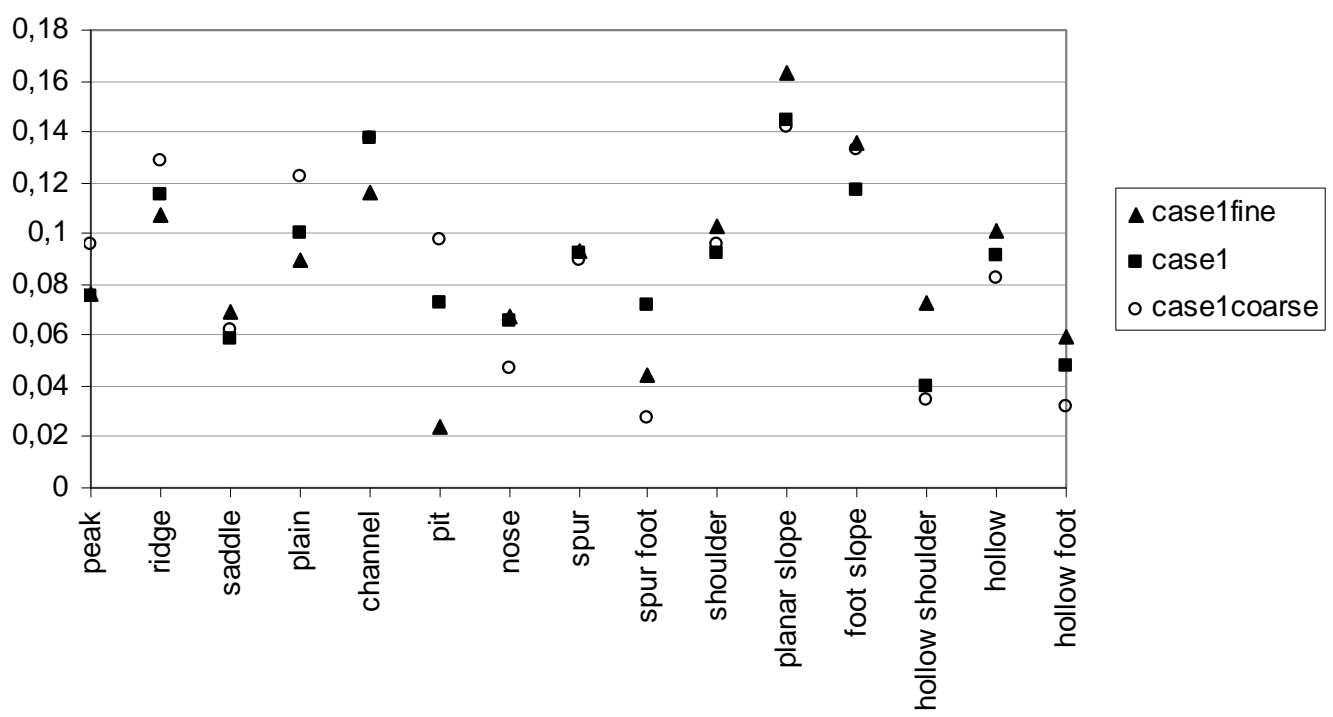

Figure 7.5: classification stability of three scales of morphometric classification of case1

\section{best classification result}

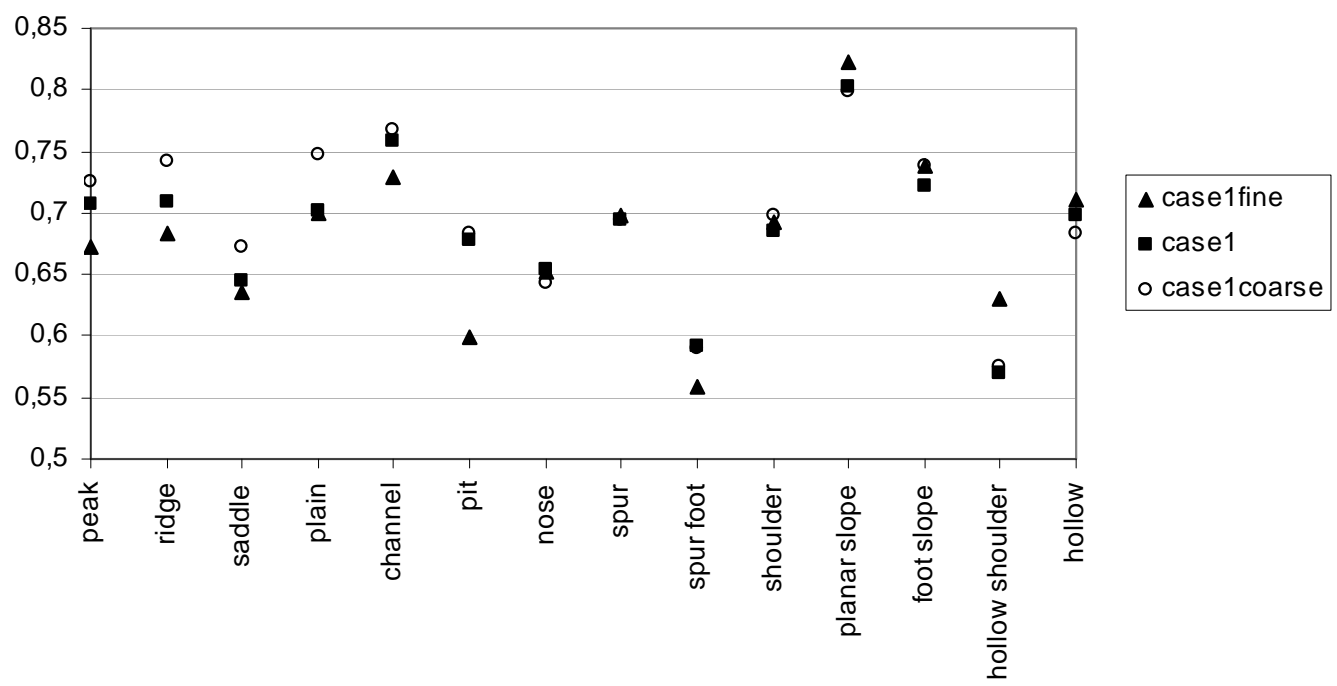

Figure 7.6: Best classification result of three scales of morphometric classification of case 1

Morphometric classification stability and efficiency measures for 'case2' vs 'case1, are given in Table 7.5. Both classification stability and best classification result is plotted for case 2 and case 1 (Figure 7.7 ,figure 7.8) and similar trend could be observed just as in the previous figures (Figure 7.5 and 7.6). 
Table 7.5: Classification stability and best classification results for morphometric classification of 'case2 and 'case1'

\begin{tabular}{|l|l|l|l|l|}
\hline & \multicolumn{2}{l|}{ Classification stability } & \multicolumn{2}{l|}{ best classification result } \\
\hline Class & case2 & case1 & case2 & case 1 \\
\hline peak & 0.088192 & 0.075648 & 0.675865 & 0.707313 \\
\hline ridge & 0.111265 & 0.114985 & 0.693366 & 0.709483 \\
\hline saddle & 0.087809 & 0.058182 & 0.672521 & 0.644185 \\
\hline plain & 0.144938 & 0.100139 & 0.797821 & 0.701385 \\
\hline channel & 0.108231 & 0.137542 & 0.7127 & 0.759198 \\
\hline pit & 0.064303 & 0.072772 & 0.662435 & 0.676863 \\
\hline nose & 0.082887 & 0.065691 & 0.699171 & 0.654359 \\
\hline spur & 0.092354 & 0.091898 & 0.689051 & 0.694762 \\
\hline spur foot & 0.053481 & 0.071403 & 0.629811 & 0.592496 \\
\hline shoulder & 0.101833 & 0.092437 & 0.696543 & 0.685619 \\
\hline planar slope & 0.124769 & 0.144923 & 0.775931 & 0.801453 \\
\hline foot slope & 0.134824 & 0.116629 & 0.729241 & 0.72242 \\
\hline hollow shoulder & 0.048766 & 0.04009 & 0.633235 & 0.568733 \\
\hline hollow & 0.08627 & 0.091103 & 0.684551 & 0.698715 \\
\hline hollow foot & 0.069219 & 0.047808 & 0.697939 & 0.642706 \\
\hline
\end{tabular}

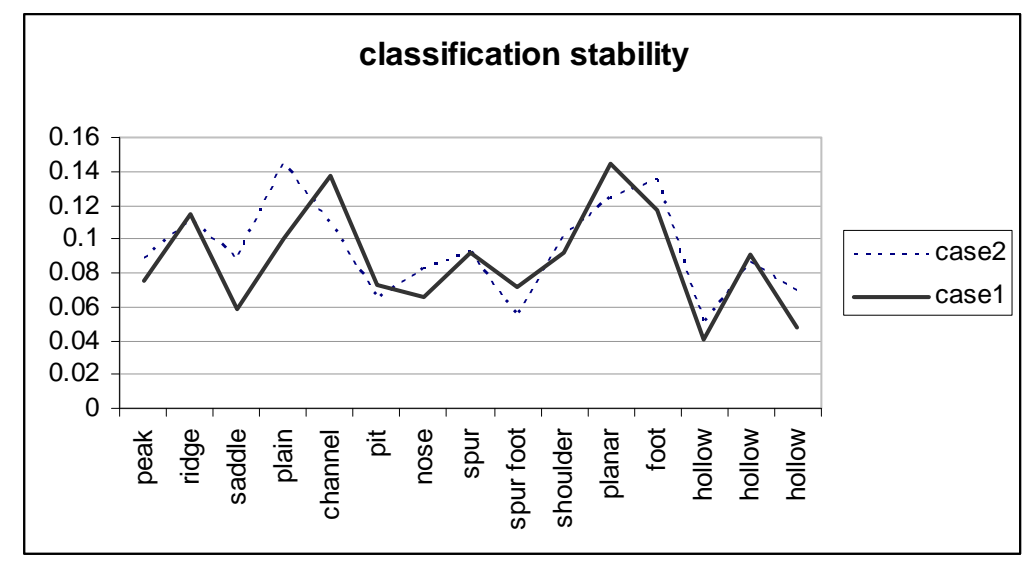

Figure 7.7: Classification stability results for morphometric classification of 'case2 and 'case1'

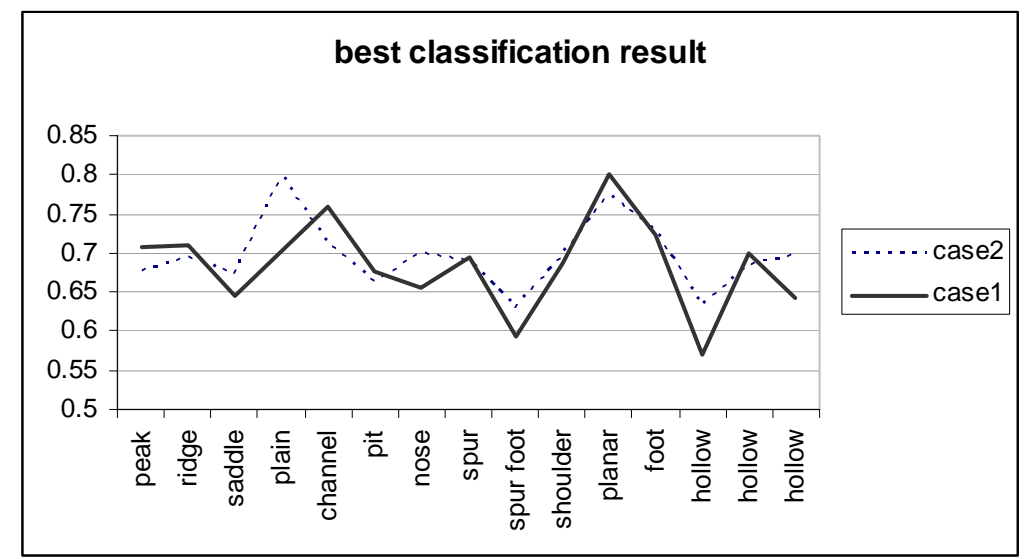

Fgure 7.8: Classification stability results for morphometric classification of 'case 2 and 'case1' 
Evaluations on stability and efficiency of classification have shown that 'planar slope', 'channel', 'foot slope', 'ridge' and 'plain forms' pose better results compared to other classes, which means these classes are better separable from the similar classes given their class definitions. On the other side 'hollow foot', 'hollow shoulder', 'spur foot', 'saddle' pose unsatisfactory classification statistics. They could be barely identified within the whole area.

\subsubsection{Geomorphometric context}

Proposed method basically comprises of two classification procedures. Initially a classification based on local geometry is performed then; geomorphometric classification through logical operators which incorporate geomorphometric context is implemented. In this stage, morphometric classes were reorganized regarding landscape context, hence morphometric classes were re classified if they were not in line with the particular organization of the landscape. Although the change is observable in between the morphometric and geomorphometric classifications, difference is quantified in this section. A 'change matrix' is calculated for morphometric vs. geomorphometric classification of 'case1' (Table 7.6) and a 3D plot is provided to illustrate amount of classes assigned to another class (Figure 7.9).

Table 7.6: 'change matrix' for morphometric classification vs geomorphometric classification of 'case1'.

\begin{tabular}{|c|c|c|c|c|c|c|c|c|c|c|c|c|c|c|c|c|}
\hline & $\begin{array}{c}\text { Geomorphometric } \\
\text { classification } \\
\text { Morphometric } \\
\text { classific ation } \\
\downarrow\end{array}$ & 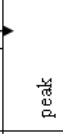 & $\begin{array}{l}\stackrel{0}{50} \\
\text { 芑 }\end{array}$ & 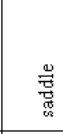 & $\begin{array}{l}\text { 䙔 } \\
\end{array}$ & $\begin{array}{l}\vec{\Xi} \\
\text { ㅁ } \\
\text { 영 }\end{array}$ & 苦 & $\begin{array}{l}\text { 㟧 } \\
\text { g. }\end{array}$ & 总 & 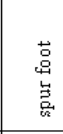 & $\begin{array}{l}\text { 岁 } \\
\text { 志 } \\
\text { 总 } \\
\end{array}$ & 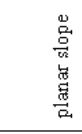 & 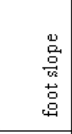 & 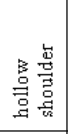 & $\begin{array}{l}\text { : } \\
\text { 总 } \\
\end{array}$ & 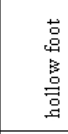 \\
\hline 1 & peak & 6085 & & & & & & 1255 & & & & & & & & \\
\hline 2 & ridge & 651 & 43451 & & & & & & 413 & & & & & & & \\
\hline 3 & saddle & & & 1780 & & 108 & & & & & & & & & & 352 \\
\hline 4 & plain & & & & 7935 & 1475 & & & & & & & & & & \\
\hline 5 & channel & & & & & 38003 & & & & & & & & & 8324 & \\
\hline 6 & pit & & & & & 4150 & 1616 & & & & & & & & 346 & \\
\hline 7 & \begin{tabular}{|l|} 
nose \\
\end{tabular} & & & & & & & 29493 & & & & & & & & \\
\hline 8 & spur & 186 & 37711 & & & & & & 119081 & 2568 & & & & & & \\
\hline 9 & spur foot & & & & & & & & & 1130 & & & & & & \\
\hline 10 & \begin{tabular}{|l} 
shoulder \\
\end{tabular} & & 5055 & & & & & & & 157 & 112588 & & & & & \\
\hline 11 & planar slope & & & & & 762 & & & & & & 291037 & 5357 & & & \\
\hline 12 & foot slope & & & & & 7710 & & & & & & 49086 & 44912 & & & \\
\hline 13 & Hollow shoulder & & & & & & & & & & & & & 893 & & \\
\hline 14 & hollow & & & & & 15460 & & & & & & & & 1095 & 124723 & 17822 \\
\hline 15 & hollow foot & & & & & 2099 & & & & & & & & & & 4161 \\
\hline
\end{tabular}




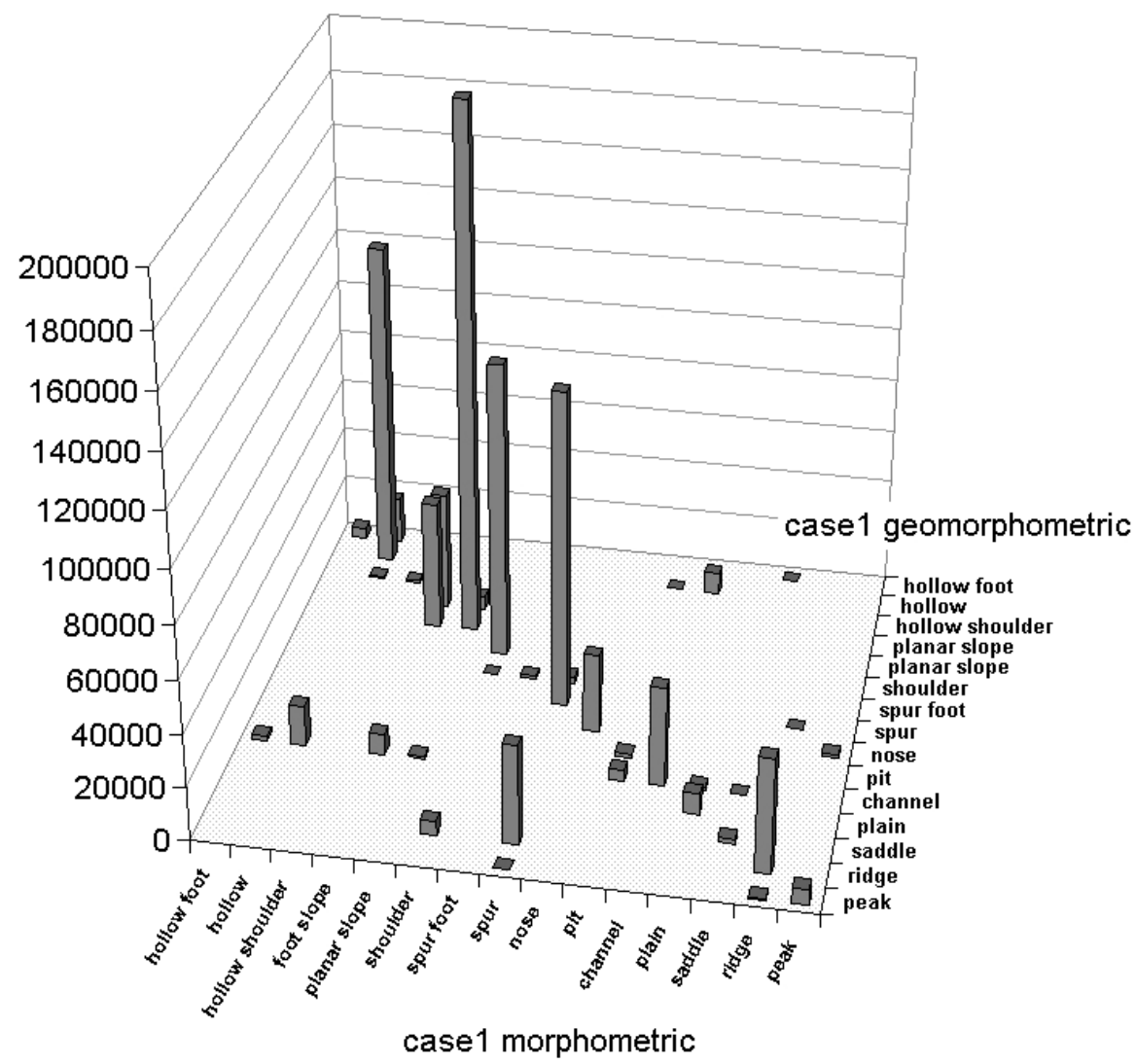

Figure 7.9: 3D plot of 'change matrix' for morphometric classification vs. geomorphometric classification of 'case1'.

To mention some of the significant changes;

Some amount of peak forms were reclassified into noses, as they do not satisfy criteria to be a peak. Some amount of plain forms were reclassified into channels as they coincide with stream lines, some amount of spur and shoulder forms were classified into ridges as they coincide with ridge lines. Some amount of planar slope form is assigned to foot slope and a big amount of foot slope form is assigned to planar slope, that is due to terrain position criteria mainly.

The amount of change and its direction (which classes are reclassified as another class after adding geomorphometric context) is quantified. However it is also important to make an evaluation of the results. As this classification is produced out of a set of logical rules, it is not fuzzy any more, therefore fuzzy statistical measures like best classification result can not be applied. Geomorphometric classifications were compared to expert suggestions and results were evaluated however given the small amount of random check points it is not likely to obtain a quantification of improvement. Therefore it is suggested that more control points are used. 


\subsubsection{Flat topography}

Method is tested on two case areas 'case1' and 'case2' with two convincingly different topographies, however both case areas are common in portraying all classes proposed (Dikau, 1989). Therefore this evaluation attempts evaluating the results of the classification on a very flat topography; 'case3' to test how the method works on a case area that portrays only a few of the classes. 'Case3' is selected form a region that is dominated by flat plains in Middle Anatolia region of Turkey. In the case area there is only a few levels including very smooth hill-like features and few minor channels with smooth beds and majority of the area is flat (Figure 7.10).

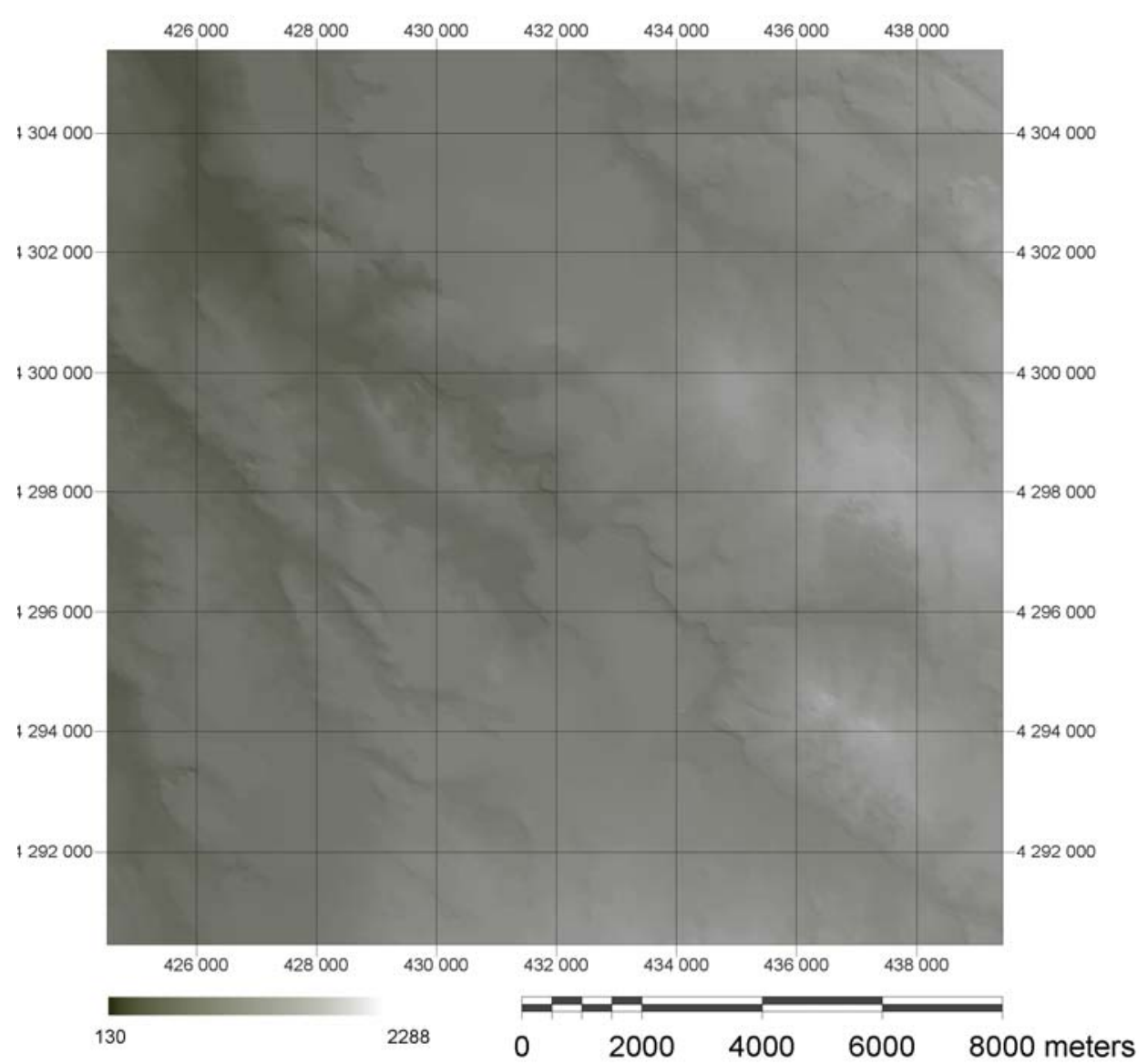

Figure 7.10: Study area: shaded relief map of 'case3'.

A geomorphometric classification was performed on the case area with same set of rules and prior data normalization. The results however depicted a mosaic as a hardly interpretable mixture of almost all classes which is not adequate as an expected outcome (Figure 7.11). 


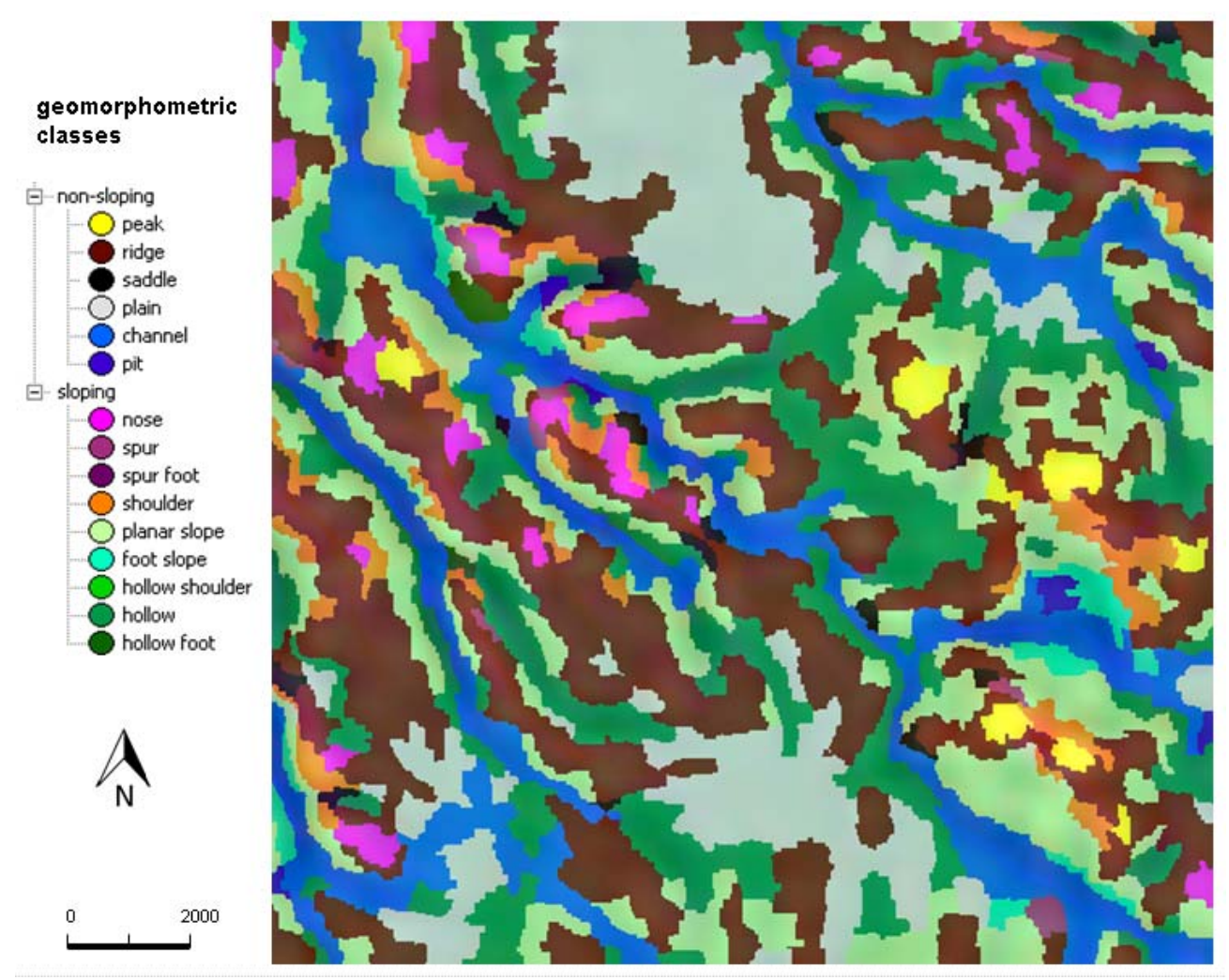

Figure 7.11: Geomorphometric classification result for 'case3'

The problem with the classification result of 'case3' is that even very smooth slope or curvature in the topography has been assigned with a relevant class, e.g. very low level areas were classified as ridges or peaks and minor river beds with very smooth dissections were classified as channels, and very smoothly sloping topography was classified as one of the sloping elements, i.e. mainly hollows for the case. The reason behind this unpleasant outcome is attributed to the normalization procedure prior to classification. 'Case1' and 'case2' and other datasets e.g. 'case1 next', 'case1coarse', 'case1 fine' were all normalized from their original values into $0-1$ or $-1+1$. This is mainly to overcome the effects that would be introduced through different data ranges belonging to different case areas or different scales for the same area. For instance maximum curvature value for 'case1' ranges in between -3.0 and 5.9 1/m and for 'case2' ranges in between -1.2 and 3.4 1/m (Table 4.2). A particular threshold should be defined to discriminate convex topography from planar or planar from concave in order to assign these area into classes like for instance 'spur', 'planar slope' or 'hollow'. If the threshold criteria for 'case1' is adopted without modifications into 'case2' then the method will underestimate the curved features as the threshold values will be very high to be satisfied in a Semantic Import model based on membership functions. But if it is modified than every time the method is applied for a new case, one will have to 
change all threshold criteria which sums up to more than ten changes in the thresholds. Therefore a normalization on the original values has been performed as explained in detail in section 6.2.1 (Classification of landforms based on local geometry) so that same threshold applies each time the classification is performed. However, if the case area has extremely low values of slope or curvature like it is for 'case3' (maximum curvature between 0.2 and $0.331 / \mathrm{m})$, then normalization produces execrated representation of terrain. As a consequence even very smooth curvature like 0.33 is attributed as maximum value for curvature. Possible solution for this problem can be setting a minimum threshold for steepness, convexity and concavity as mentioned in section 2.2.3.1 (Morphometry) as the minimum value for normalization instead of mean in case the turn over value determined using statistical mean is lower than this minimum value.

\subsubsection{Classification Consistency}

Two case areas 'case1' and 'case1next' with and overlapping area of $8 \times 8 \mathrm{~km}$ are classified via same classification method with the same rule set independently. Then overlapping areas from both 'case1' and 'case1next' are extracted and differences were evaluated. Results depict $\% 65$ of identical classification and $\% 35$ of the area is classified differently in both cases, which is not a slight amount (Figure 7.12). However most of this difference in classification is considered to be due to segmentation which creates different object boundaries for each case area. For instance it is clearly observed that there are differently classified regions along the rivers and ridges due to different boundary conditions created for channels and ridges in both of the cases. For instance in 'caselnext' part of an area corresponding to 'channel' is classified as 'foot slope' in 'case1' that accompany channels and a misclassification occurred. Another observation is that misclassified areas tend to be the second best classification of the mentioned areas. It is considered that classification will pose slight amount of difference if the scale of segmentation is reduced. With a smaller scale parameter, segmentation will pose smaller objects that can flexibly represent class descriptions and they will tend to have less amount of spatial differences in boundaries socalled epsilon distance. 


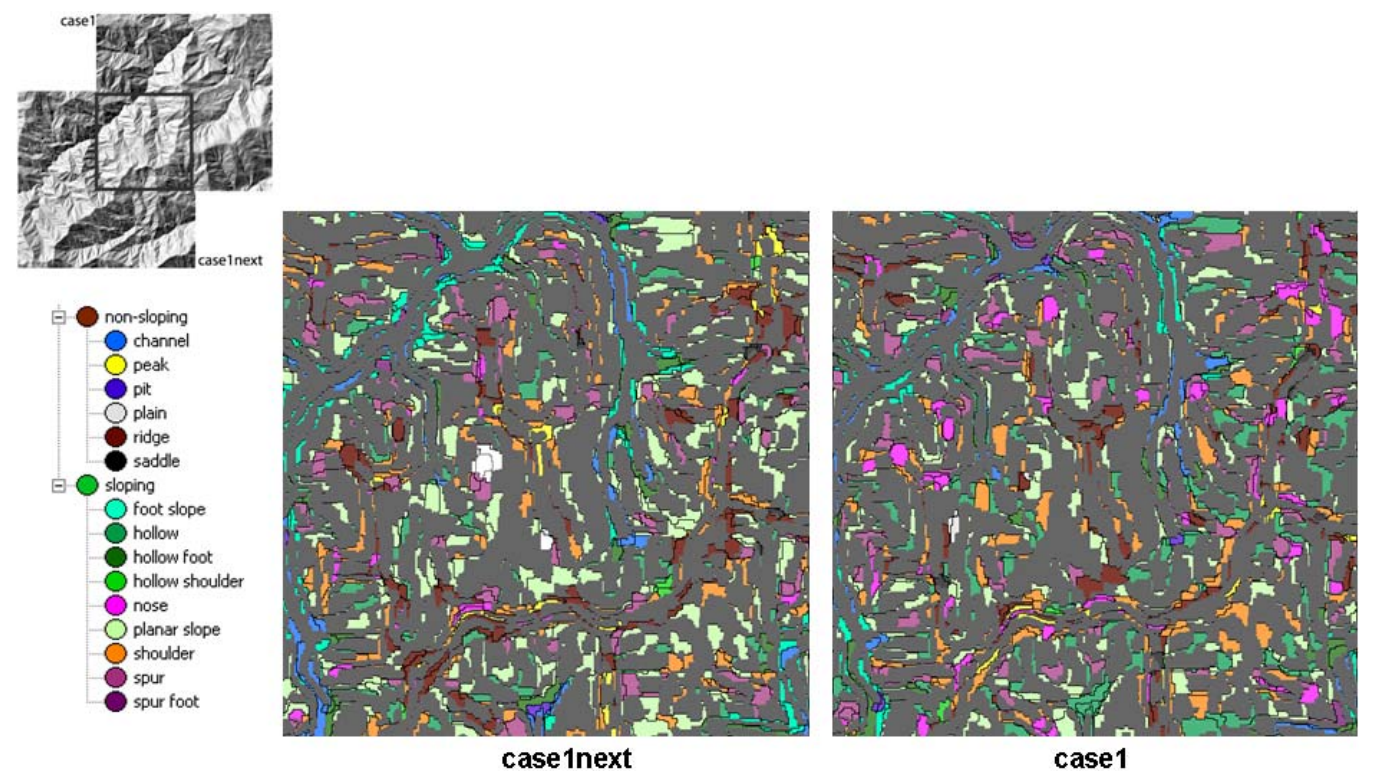

Figure 7.12: Overlapping area classified as a part of morphometric classification of 'case1next' (left) and 'case1' (right). Objects with the same classes are all assigned with gray color this area covers $\% 65$ of the total area

\subsubsection{External evaluation}

An evaluation on classification results is also implemented with the help of some geographers and geomorphologists experienced at landform classification who volunteered making an evaluation on a set of GIS layer pertaining to two case areas; 'case1' and 'case2'. This evaluation involves comparison of proposed classification method with the expert knowledge over a set of random locations on two case areas (Figure 7.13).

Stratified random points are generated using random point generator script on ArcView given the class boundaries, so that each class can be represented with enough number of points even if they are relatively very small in extents. A total of 85 points for each case are produced.

A dataset that includes DEM, shaded relief, contours, objects from the segmentation and random points is prepared and a grid is provided that represent the window size used for calculating DTMs that is $45 \times 45(675 \times 675 \mathrm{~m})$ window. Volunteered scientists are provided with this dataset for two case areas and an explanatory document which describes the method, classes and the scale of the study. Explanatory document provided to the scientists is given in Appendix F. Six scientists have returned their evaluations, however two of them 
were omitted as their evaluations involved some inconsistencies that can not be attributed to a deficiency in the model proposed: e.g. suggesting a form is hollow rather than a spur where it is very clear that the form has convexity in plan curvature. One of the interpreters noted that he evaluated at a fine scale, the other one is thought to be misinterpreting the form of terrain. Results from four evaluators for 'case1' and three for 'case2'are given in Table 7.7 and Table 7.8 respectively.
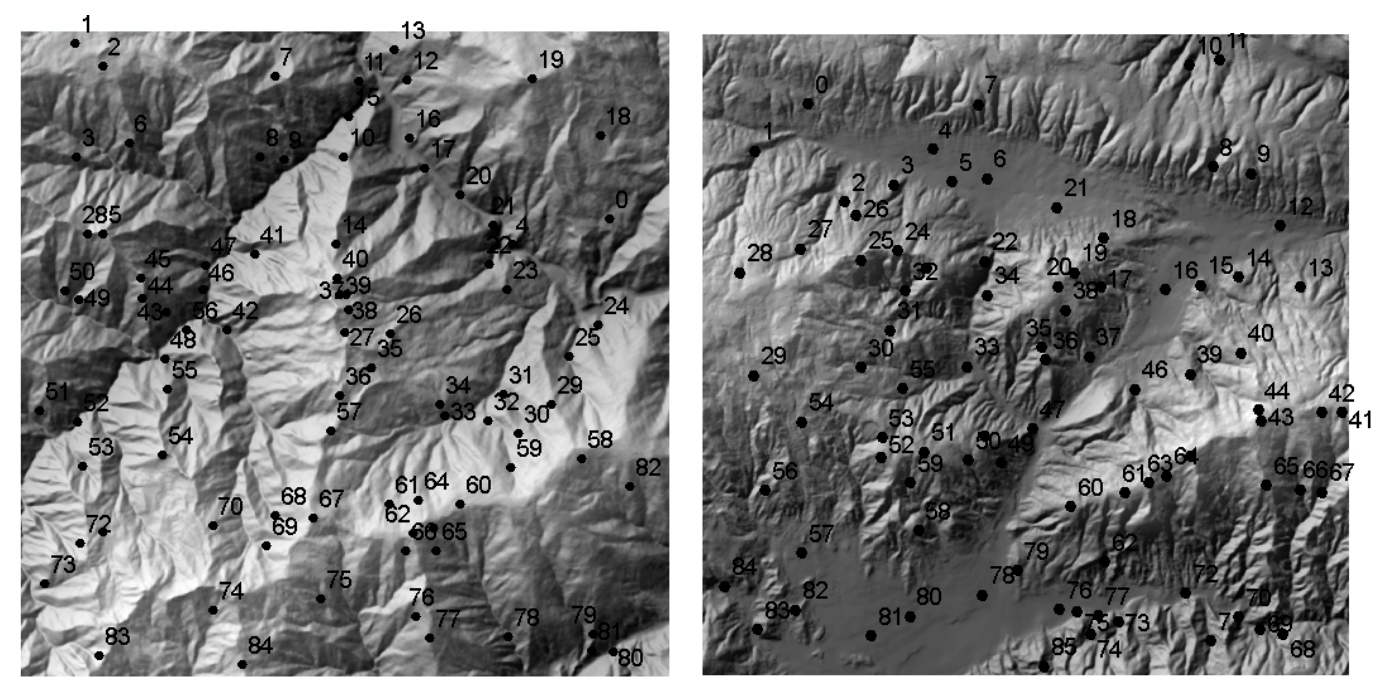

Figure 7.13: Stratified random points on case areas: 'case1'(left) and, 'case2' (right).

Table 7.7: Evaluation results compared with suggestions from four scientists for case1 Exact matches are highlighted in yellow, close matches are highlighted in fair yellow and total mismatch is with red font

\begin{tabular}{|l|r|l|l|l|l|}
\cline { 3 - 6 } \multicolumn{2}{l|}{} & \multicolumn{5}{c|}{ Evaluators } \\
\hline $\begin{array}{l}\text { Classification } \\
\text { result }\end{array}$ & ID & \multicolumn{1}{c|}{ J.Schmidt } & \multicolumn{1}{c|}{ L.Dragut } & \multicolumn{1}{c|}{ B.Rojay } & C.Eisank \\
\hline Plain & 0 & Hollow & Hollow & Hollow foot & Hollow shoulder \\
\hline Plain & 1 & Planar slope & Hollow foot & Planar slope & Hollow \\
\hline Plain & 2 & Planar slope & Plain & Shoulder & Plain \\
\hline Spur foot & 3 & Ridge & Spur & Spur & Spur \\
\hline Spur foot & 4 & Ridge & Nose & Nose & Nose \\
\hline Peak & 5 & Peak & Peak & Shoulder & Planar slope \\
\hline Nose & 6 & Planar slope & Peak & Nose & Shoulder \\
\hline Saddle & 7 & Planar slope & Planar slope & Planar slope & Pit \\
\hline Planar slope & 8 & Planar slope & Planar slope & Planar slope & Planar slope \\
\hline Hollow foot & 9 & Spur & Planar slope & Nose & Hollow \\
\hline Shoulder & 10 & Planar slope & Planar slope & Planar slope & Planar slope \\
\hline Spur & 11 & Shoulder & Nose & Nose & Nose \\
\hline Channel & 12 & Hollow & Hollow & Hollow & Hollow \\
\hline Spur & 13 & Hollow shoulder & Nose & Spur & Hollow shoulder \\
\hline Ridge & 14 & Saddle & Saddle & Ridge & Saddle \\
\hline Channel & 15 & Channel & Channel & Channel & Channel \\
\hline
\end{tabular}


Table 7.7 cont'd.

\begin{tabular}{|c|c|c|c|c|c|}
\hline $\begin{array}{l}\text { Classification } \\
\text { result }\end{array}$ & ID & J.Schmidt & L.Dragut & B.Rojay & C.Eisank \\
\hline Foot slope & 16 & Planar slope & Foot slope & Planar slope & Foot slope \\
\hline Channel & 17 & Plain & Plain & Plain & Plain \\
\hline Planar slope & 18 & Planar slope & Planar slope & Planar slope & Planar slope \\
\hline Peak & 19 & Peak & Peak & Ridge & Peak \\
\hline Pit & 20 & Plain & Plain & Foot slope & Hollow foot \\
\hline Foot slope & 21 & Spur & Nose & Planar slope & Spur \\
\hline Channel & 22 & Planar slope & Hollow & Hollow & Foot slope \\
\hline Nose & 23 & Spur & Nose & Spur & Nose \\
\hline Foot slope & 24 & Spur & Spur foot & Hollow foot & Foot slope \\
\hline Foot slope & 25 & Spur foot & Planar slope & Hollow & Spur foot \\
\hline Ridge & 26 & Spur & Spur & Spur & Shoulder \\
\hline Hollow shoulder & 27 & Planar slope & Pit & Hollow shoulder & Pit \\
\hline Saddle & 28 & Saddle & Pit & Hollow shoulder & Saddle \\
\hline Spur & 29 & Planar slope & Nose & Spur & Spur \\
\hline Spur & 30 & Spur & Spur & Spur foot & Spur \\
\hline Hollow foot & 31 & Planar slope & Spur foot & Hollow & Spur foot \\
\hline Hollow foot & 32 & Planar slope & Pit & Spur foot & Shoulder \\
\hline Planar slope & 33 & Planar slope & Planar slope & Planar slope & Planar slope \\
\hline Shoulder & 34 & Hollow shoulder & Hollow & & Hollow \\
\hline Hollow & 35 & Planar slope & Planar slope & Planar slope & Planar slope \\
\hline Nose & 36 & Nose & Nose & Nose & Nose \\
\hline Nose & 37 & Spur & Nose & Nose & Spur \\
\hline Hollow shoulder & 38 & Hollow & Pit & Hollow shoulder & Hollow \\
\hline Hollow shoulder & 39 & Planar slope & Pit & Hollow shoulder & Hollow foot \\
\hline Peak & 40 & Peak & Peak & Peak & Peak \\
\hline Hollow & 41 & Planar slope & Hollow & Hollow & Planar slope \\
\hline Pit & 42 & Planar slope & Foot slope & Planar slope & Hollow foot \\
\hline Planar slope & 43 & Planar slope & Planar slope & Planar slope & Hollow \\
\hline Nose & 44 & Spur & Spur & Nose & Nose \\
\hline Peak & 45 & Planar slope & Shoulder & Planar slope & Planar slope \\
\hline Foot slope & 46 & Planar slope & Planar slope & Planar slope & Planar slope \\
\hline Pit & 47 & Planar slope & Spur foot & Nose & Hollow foot \\
\hline Pit & 48 & Hollow & Hollow foot & Hollow foot & Channel \\
\hline Spur & 49 & Spur & Nose & Hollow & Spur foot \\
\hline Spur foot & 50 & Spur & Nose & Spur & Spur \\
\hline Planar slope & 51 & Planar slope & Planar slope & Spur & Nose \\
\hline Channel & 52 & Channel & Channel & Channel & Channel \\
\hline Hollow & 53 & Hollow & Hollow & Hollow foot & Channel \\
\hline Peak & 54 & Planar slope & Peak & Peak & Peak \\
\hline Nose & 55 & Spur & Nose & Nose & Spur \\
\hline Foot slope & 56 & Planar slope & Foot slope & Planar slope & Nose \\
\hline Ridge & 57 & Nose & Ridge & Saddle & Ridge \\
\hline Shoulder & 58 & Planar slope & Shoulder & Shoulder & Hollow shoulder \\
\hline Hollow & 59 & Planar slope & Hollow & Hollow & Channel \\
\hline Saddle & 60 & Saddle & Saddle & Saddle & Saddle \\
\hline Planar slope & 61 & Planar slope & Planar slope & Hollow shoulder & Nose \\
\hline Peak & 62 & Nose & Peak & Peak & Peak \\
\hline
\end{tabular}


Table 7.7 cont'd.

\begin{tabular}{|l|r|l|l|l|l|}
\hline $\begin{array}{l}\text { Classification } \\
\text { result }\end{array}$ & ID & \multicolumn{1}{|c|}{ J.Schmidt } & \multicolumn{1}{c|}{ L.Dragut } & \multicolumn{1}{c|}{ B.Rojay } & \multicolumn{1}{c|}{ C.Eisank } \\
\hline Shoulder & 63 & Planar slope & Shoulder & Shoulder & Planar slope \\
\hline Spur & 64 & Planar slope & Spur & Spur & Shoulder \\
\hline Planar slope & 65 & Planar slope & Planar slope & Planar slope & Planar slope \\
\hline Hollow shoulder & 66 & Hollow & Pit & Hollow & Hollow shoulder \\
\hline Peak & 67 & Peak & Peak & Peak & Peak \\
\hline Hollow & 68 & Hollow & Hollow shoulder & Hollow & Channel \\
\hline Hollow & 69 & Planar slope & Hollow & Hollow & Foot slope \\
\hline Shoulder & 70 & Nose & Shoulder & Planar slope & Shoulder \\
\hline Planar slope & 71 & Hollow & Hollow shoulder & & Planar slope \\
\hline Shoulder & 72 & Planar slope & Shoulder & Shoulder & Planar slope \\
\hline Shoulder & 73 & Spur & Nose & Planar slope & Planar slope \\
\hline Hollow & 74 & Hollow & Hollow & Hollow & Hollow \\
\hline Planar slope & 75 & Hollow & Hollow & Hollow & Hollow \\
\hline Hollow shoulder & 76 & Planar slope & Hollow shoulder & Hollow & Planar slope \\
\hline Spur & 77 & Nose & Spur & Spur & Spur \\
\hline Channel & 78 & Planar slope & Spur foot & Channel & Foot slope \\
\hline Spur & 79 & Spur & Spur & Spur & Spur \\
\hline Channel & 80 & Channel & Plain & Plain & Plain \\
\hline Spur foot & 81 & Planar slope & Spur foot & Planar slope & Hollow \\
\hline Spur & 82 & Spur & Spur & Spur & Spur \\
\hline Hollow shoulder & 83 & Spur & Hollow shoulder & Planar slope & Spur \\
\hline Ridge & 84 & Spur & Saddle & Ridge \\
\hline
\end{tabular}

There are 12 exact matches by four of the interpreters for case 1 out of 85 points and a total of 42 points were agreed on by more than half of the interpreters.

Table 7.8: Evaluation results compared with suggestions from four scientists for case2. Exact matches are highlighted in yellow, close matches are highlighted in fair yellow and total mismatch is with red font

\begin{tabular}{|l|r|l|l|l|}
\cline { 3 - 5 } \multicolumn{2}{l|}{} & \multicolumn{3}{c|}{ Evaluators } \\
\hline $\begin{array}{l}\text { Classification } \\
\text { result }\end{array}$ & ID & J.Schmidt & L.Dragut & C.Eisank \\
\hline pit & 1 & Spur & Foot slope & Hollow foot \\
\hline planar slope & 2 & Spur & Spur foot & Nose \\
\hline spur & 3 & Spur & Spur foot & Spur foot \\
\hline plain & 4 & Planar slope & Planar slope & Planar slope \\
\hline channel & 5 & Planar slope & Plain & Channel \\
\hline plain & 6 & Planar slope & Planar slope & Planar slope \\
\hline shoulder & 7 & Spur & Spur & Nose \\
\hline hollow & $\mathbf{8}$ & Hollow & Hollow & Hollow \\
\hline shoulder & 9 & Nose & Nose & Nose \\
\hline hollow foot & 10 & Planar slope & Hollow & Hollow \\
\hline spur & 11 & Planar slope & Spur foot & Spur \\
\hline channel & 12 & Plain & Plain & Channel \\
\hline
\end{tabular}


Table 7.8 cont'd.

\begin{tabular}{|c|c|c|c|c|}
\hline $\begin{array}{l}\text { Classification } \\
\text { result }\end{array}$ & ID & J.Schmidt & L.Dragut & C.Eisank \\
\hline spur & 13 & Spur & Spur & Spur \\
\hline nose & 14 & Planar slope & Spur foot & Nose \\
\hline spur foot & 15 & Spur & Spur foot & Spur foot \\
\hline plain & 16 & Channel & Planar slope & Saddle \\
\hline hollow & 17 & Hollow & Hollow & Channel \\
\hline planar slope & 18 & Planar slope & Planar slope & Planar slope \\
\hline ridge & 19 & Nose & Spur & Spur \\
\hline peak & 20 & Peak & Peak & Peak \\
\hline plain & 21 & Planar slope & Foot slope & Spur foot \\
\hline spur foot & 22 & Planar slope & Nose & Shoulder \\
\hline hollow shoulder & 23 & Spur & hollow shoulder & hollow shoulder \\
\hline ridge & 24 & Planar slope & Shoulder & Peak \\
\hline nose & 25 & Peak & Peak & Peak \\
\hline spur & 26 & Spur & Spur & Spur \\
\hline hollow & 27 & Hollow & Hollow & Channel \\
\hline planar slope & 28 & Planar slope & Shoulder & hollow shoulder \\
\hline planar slope & 29 & Planar slope & Planar slope & Nose \\
\hline hollow & 30 & Hollow & Hollow & Hollow \\
\hline saddle & 31 & Saddle & Saddle & Saddle \\
\hline pit & 32 & Spur foot & Nose & Spur foot \\
\hline pit & 33 & Planar slope & Foot slope & Foot slope \\
\hline foot slope & 34 & Hollow & Foot slope & Foot slope \\
\hline saddle & 35 & Saddle & Saddle & Saddle \\
\hline peak & 36 & Peak & Peak & Peak \\
\hline foot slope & 37 & Planar slope & Foot slope & Spur foot \\
\hline hollow shoulder & 38 & Hollow & Pit & Pit \\
\hline spur & 39 & Spur & Spur & Spur \\
\hline ridge & 40 & Spur & Spur & Spur \\
\hline shoulder & 41 & Planar slope & Shoulder & Shoulder \\
\hline shoulder & 42 & Planar slope & Shoulder & hollow shoulder \\
\hline ridge & 43 & Planar slope & Ridge & Ridge \\
\hline shoulder & 44 & Planar slope & Shoulder & Shoulder \\
\hline nose & 45 & Planar slope & hollow shoulder & Shoulder \\
\hline hollow foot & 46 & Planar slope & Hollow foot & Channel \\
\hline spur foot & 47 & Spur foot & Spur foot & Spur foot \\
\hline shoulder & 48 & Planar slope & Planar slope & Planar slope \\
\hline planar slope & 49 & Planar slope & Nose & Spur \\
\hline hollow shoulder & 50 & Hollow & Pit & Hollow \\
\hline peak & 51 & Planar slope & Shoulder & Peak \\
\hline nose & 52 & Planar slope & Shoulder & Shoulder \\
\hline saddle & 53 & Hollow & hollow shoulder & hollow shoulder \\
\hline channel & 54 & Planar slope & Shoulder & hollow shoulder \\
\hline planar slope & 55 & Planar slope & Pit & Planar slope \\
\hline spur & 56 & Hollow & Pit & Nose \\
\hline plain & 57 & Plain & Foot slope & Planar slope \\
\hline nose & 58 & Planar slope & Shoulder & Shoulder \\
\hline hollow & 59 & Planar slope & Hollow & Hollow \\
\hline
\end{tabular}


Table 7.8 cont'd.

\begin{tabular}{|c|c|c|c|c|}
\hline $\begin{array}{l}\text { Classification } \\
\text { result }\end{array}$ & ID & J.Schmidt & L.Dragut & C.Eisank \\
\hline nose & 60 & Planar slope & Nose & Nose \\
\hline ridge & 61 & Planar slope & Planar slope & Nose \\
\hline hollow & 62 & Planar slope & Hollow & \\
\hline peak & 63 & hollow shoulder & Peak & Peak \\
\hline hollow shoulder & 64 & Planar slope & hollow shoulder & Hollow \\
\hline nose & 65 & Planar slope & Spur & Nose \\
\hline hollow foot & 66 & Hollow & Hollow & Channel \\
\hline hollow foot & 67 & Channel & Hollow & Channel \\
\hline foot slope & 68 & Planar slope & Foot slope & Hollow \\
\hline channel & 69 & Planar slope & Channel & Channel \\
\hline spur foot & 70 & Spur & Spur foot & Spur foot \\
\hline hollow foot & 71 & Planar slope & Hollow & Channel \\
\hline channel & 72 & Plain & Plain & Channel \\
\hline hollow & 73 & Planar slope & Hollow foot & Channel \\
\hline pit & 74 & Channel & Hollow & Channel \\
\hline foot slope & 75 & Plain & Foot slope & Plain \\
\hline channel & 76 & Plain & Plain & Plain \\
\hline foot slope & 77 & Planar slope & Foot slope & Foot slope \\
\hline plain & 78 & Plain & Foot slope & Foot slope \\
\hline ridge & 79 & Spur & Spur foot & Spur \\
\hline plain & 80 & Plain & Foot slope & Foot slope \\
\hline channel & 81 & Plain & Foot slope & Foot slope \\
\hline ridge & 82 & Ridge & Spur foot & Peak \\
\hline saddle & 83 & Hollow & Foot slope & Hollow foot \\
\hline foot slope & 84 & Planar slope & Foot slope & Foot slope \\
\hline saddle & 85 & Saddle & Saddle & Saddle \\
\hline
\end{tabular}

There are 13 exact matches by four of the interpreters for case 1 out of 85 points and a total of 28 points were agreed on by two or more of the interpreters.

Results show that only $15 \%$ and $17 \%$ of the points were agreed to be the same classification as the proposed classification for case 1 and case 2 respectively and about $50 \%$ of the points were agreed to be the same for the two case areas. VanWesten (1993) who made a similar assessment reported that variability on mapping geomorphologic units via photointerpretation showed that only $10 \%$ of the area was assigned the same class by four interpreters. About $17 \%$ was mapped identically by three and $53 \%$ by two interpreters. Results obtained for the classification are similar and even better than reported in the study of VanWesten (1993). 
Besides matches, another aspect to be considered is the complete mismatches where all scientists agree on a particular landform different than the classification result. There are 5 such cases for 'case1' and four for 'case2' are in red fonts in Table 7.7 and 7.8. However there is no specific tendency in these mismatches. The inconsistency is attributed to specific cases at particular locations, like for instance a 'channel' at that location is rather smooth that the evaluators assigned it to 'planar slope'.

Another feedback from the evaluators was that 'case2' was more difficult to interpret compared to 'case1' which is attributed to more roughness of the 'case2' topography.

Regarding the special properties of landforms that have indeterminate boundaries and gradually changing attributes from one class to another, they should not be thought of as crisp classes. More over they are semantically driven and have high scale dependencies where each observer tend to pose different evaluations to an acceptable extent. Therefore standard accuracy assessment procedures widely applied on image classification results are not favorable to be adopted for landform classification. A weighted accuracy measurement is considered to better portray similarities vs. differences in classification results and evaluations from external sources like expert knowledge in this study or field observations that are prone to scale effect as well.

Figure 7.14 represents anticipated organization of classes across a part of a landscape. Classes are known to depict similarities in attribute dimension and proximity in spatial dimension, however some classes depict more. This idea constructs the basis of making a weighted evaluation.

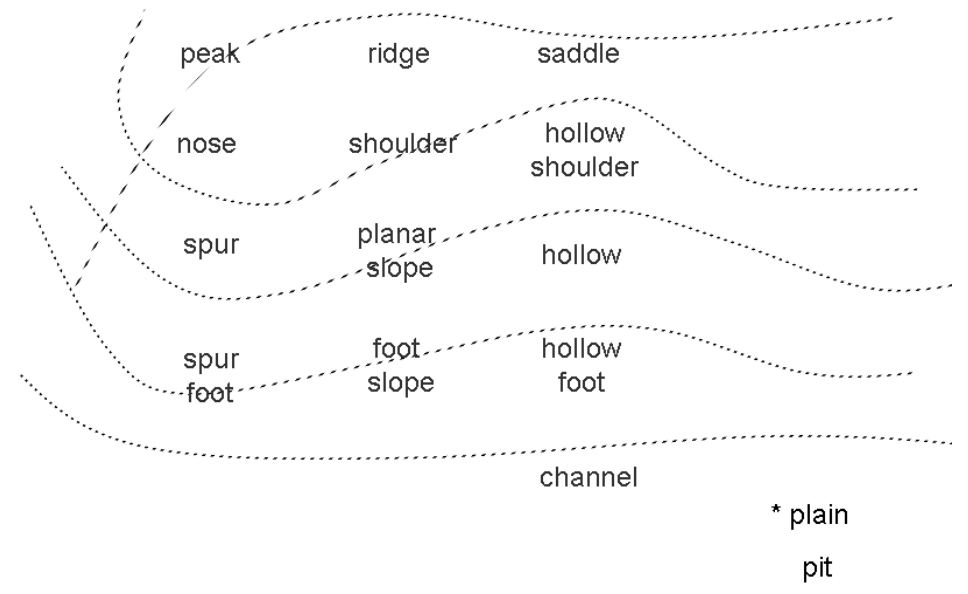

Figure 7.14: representation of anticipated landforms across the landscape. Plain ant pit are not inline with particular organisation 
Figure 7.15 is intended to represent gradual similarity of proposed classes. Accordingly 'nose' and 'spur foot' that typically form the upper and the lower parts of a wider spur and are very similar to class 'spur'. Similarly 'hollow shoulder' and 'hollow foot' that typically form the upper and the lower parts of a wider hollow and are very similar to class 'hollow'. For instance; if a classification result for an object is 'hollow foot' and it is suggested as 'hollow' there is less misclassification compared to a 'hollow foot' suggested to be a 'hollow shoulder' or 'spur foot'. According to attribute similarity, proximity and visual interpretation capabilities observed, an anticipated scheme that represents weights with color is suggested (Figure 7.15).

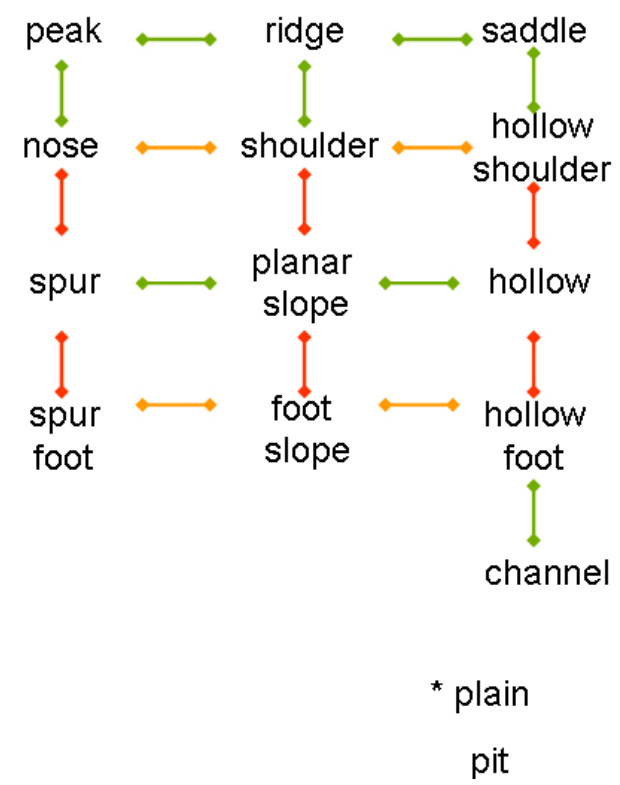

Figure 7.15: An anticipated weights of similarity of landform classes. Red is the exact match, orange is close match and green is fair match

Evaluations from the interpreters that can be constituted on the basis of this idea; i.e. comparing the results in their graded match; a more flexible and representative consistency check can be made rather than rigid comparisons used in standard accuracy check. However weighing the match between the classes is then of concern, as these weight coefficients would be subjective. A possible solution might be to use measures on similarity, e.g. fuzzy statistics of classes that is; weighting according to second best, third best classification, for instance; if second best classification result for peak is ridge on over all classification result, then, ridge can be weighted a as close match. 


\subsection{Discussion of the Method}

Method can be discussed both in terms of its basic definitions and in terms of the analysis methods and selection of the criteria. To start with the basic definitions that were described in detail in Chapter 3 (Method of Landform Classification), scale of the study and the classes constitute the basics of the classification method for this study.

Scale: Landscape scale where majority of the processes significant to human takes place is adopted as the scale for this study. However, definitions for the landscape scale are not very clear. There is hardly any references that describe the limits of landscape scale. Some information from studies in the literature that were supposed to work at landscape scale were referred and landscape scale is accepted as a scale range in between micro relief and mesorelief that was more definitely described. Scale is determined by the window of perception and scale for grid datasets that are comprised of regular pixels are usually handled with a window of nxn pixels that compute and attribute moving towards the whole scene. Scale therefore for this study is handled in pixel domain although there is an alternative way of handling scale in object domain in an OBIA system. There are several studies especially in landscape classification where scale is handled as nested hierarchies of objects where each object level represents a specific scale. Accordingly a low level object layer generated through fine scale segmentation represents for instance microrelief, and a higher level object layer which is generated through a coarser segmentation that aggregates objects from the lower level would represent mesorelief classes. However the challenge is combining classes from the lower level to the higher level into meaningful classes. There is research on handling scale in object domain for a nested hierarchy of landforms. This study as implemented at a specific scale that is Mesorelief $\mathrm{A}$, and for evaluation of scale effect purposes it was implemented at a finer and a coarser scale however al those scales were compatible and constituted lower and the upper limits of the specified scale hence produced same set of classes. As a consequence representation of scale at pixel domain is considered adequate for this study.

Classes: Generic landform elements can be exercised from a perspective of a variety of application disciplines, however human perception was taken as the basis as landscape scale is related with the processes significant to human. Therefore, a classification scheme that reveals general geomorphometry of the terrain and that is generic and exhaustive is intended to be adopted for this study. Among many classification schemes that reveal form, position 
or both, Dikau's (1989) is considered to be the most representative for general geomorphometry and 15 classes described in this scheme are considered generic and perceived by people even out of earth sciences. There are many classification schemes in the literature that represent geomorphometric classes. Widely used classification schemes described in section 2.2.3.2.1 (Terrain Position) are adequate when gravity-induced processes are considered, like e.g. runoff. However, they might be very little related to landform perception, and to applicability in non-gravity processes. Therefore a general purpose scheme is adopted. There are mainly three schemes of this type: scheme by Peucker and Douglas (1974), Dikau (1989) and Shary (2005) as described in section 2.2.3.1 (Morphometry). Among these Peucker and Douglas's scheme (1974) was oversimplified with 6 classes where 5 represents non-sloping elements and there is only one class that represents sloping features. Shary's scheme (2005) is complicated with 12 classes for sloping elements and non-sloping features were not represented at all. Dikau's scheme however represents 9 sloping and 6 non-sloping features that are all possible combinations of slope and curvature.

Pre-process: DTMs that are derived out of DEMs comprise the basic dataset used for this study. DTMs include morphometric and geomorphometric parameters that pertain to landscape. Slope and curvature DTMs; plan, profile, maximum and minimum curvature efficiently represents form. However, normalization of these DTMs prior to classification besides its advantages has critical aspects as evaluated in section 7.1.4 (Evaluation of classification on a flat topography). When the slope or curvature values are extremely low for a particular case a minimum threshold should be adopted. Topographic Position Index (TPI) generated for the cases is subdivided into three positions. However, threshold criteria for division is still of concern and can be related to a formal background on relative terrain position, perhaps adopting a run-off model. Extraction of terrain network features; streams and ridges, especially ridges can be improved. Use of an inverse DEM is not a very formal way of extracting ridges however aster a series of modification regarding more formally derived basin divides, helped extracting main ridges for the study.

Segmentation: A series of trial and error progress have been implemented for the segmentation of the input data with different segmentation parameters and possible combinations of different segmentation parameters. Parameters involve input weights, scale, homogeneity measures moderated by scale that are shape vs. color and compactness vs. smoothness as described in detain in section 2.4.1 (Segmentation). This trial and error 
procedure provided important information about the effect of parameter change. Accordingly it is evident that scale identified the sizes of objects which could be considered from a minimum mapping unit / sampling resolution perspective. Input weight has a direct effect on the object shapes where the objects depicted forms that better fit to the input with a higher weight. For instance when the weight of slope is increased objects portray contourlike forms that is characteristic of DTM: slope. Weight of shape vs. color was kept at 0.1 where weight of both shape and color should sum up to 1 . This yielded rather compact objects. This is especially important for further classification where attribute similarity is the basis for classification criteria. If the weight of shape vs color is increased homogeneity criterion becomes less dependent on attribute information of DTMs that produce misrepresentative segments of terrain form. That is considered to be related with the continuous nature of terrain which doesn't pose some regular 2D shapes as could be observed in the satellite imagery.

Scale for the segmentation of morphometric DTMs after a trial and error was considered to be best represented within the range of 5 to 12 for the landscape scale. Scale lower than 5 produced very fine segmentation that is just one step beyond pixels, and higher than 12 produced segments with more than one form property. However, deciding on a single best value for scale parameter is not very easy via visually interpreting the objects vs. input and a formal framework that is based on statistics is needed. An optimal scale where a higher or lower scale than that is less representative should be obtained. Local variance (LV) introduced by Woodcock and Strahler (1987). for instance was being used for determining an optimal resolution for particular imagery.

Dragut et al. (2010) developed a technique for estimating the scale parameter in image segmentation of remotely sensed data using an estimation function called Estimation of Scale Parameter (ESP) that builds on idea of LV. 'Rate of Change (ROC) of LV' plotted against 'scale' depict that first levelling out for the curve is at scale: 8 therefore scale should be 8 for the cases. For fine and coarse scales of 'case1' it is 7 and 9 respectively. Actually the curve did not exactly level out at these scales but tended to level out (Figure 6.3 to 6.6). These scales obtained with ESP tool are considered to well represent the variability of form attributes. However, when the procedure is followed with classification based on attributes as in this study slightly smaller objects that are more homogeneous can help better representation of class boundaries. ESP can evaluate single input at a time and slope is evaluated for scale estimation. However ESP is further being improved for evaluating multiple inputs at a time which can produce better estimations of scale given input layers. 
Classification: Classification for the study mainly comprises of two parts; initially, objects are classified based on local geometry, and fuzzy 'morphometric classes' are obtained. Then, 'morphometric classes' and geomorphometric context that is represented with 'relative terrain position' and 'terrain network' are classified with a set of logical rules to end up with 'geomorphometric classes'. Fuzzy representation of landforms is quite adequate for representing contiguous landform classes, morphometric classed that are derived using SI model produces fuzzy classes however, geomorphometric classes are crisp because of the logical rule sets, and rule sets seems to be the proper way or bringing to gather morphometric classes and geomorphometric context represented by existence data, i.e. ridges and streams. An improved version of this method could be the one that produces fuzzy geomorphometric classes.

Landform classification studies usually classify terrain both in terms of form and position at the same time. However proposed classification method utilises separate classification of form and position where one can reach either form or position through object based hierarchy and make independent adjustments.

Use of terrain network features for geomorphometric classification on the other side is not very common. Terrain network graphs like Pfaltz are better suited to represent hierarchies, which otherwise are frequently neglected. In this study one of the rules of graph-based approach on relationship between ridge lines and peak points is adopted. However this study presents a loose coupling of form, position and terrain network and the method can be improved to a more sophisticated and structured system of landform organization.

Terrain on the other side is assumed rather regular and oversimplified in this study, as for instance there is an order of classes from up to bottom and in the horizontal sequence as well, however terrain may not always fit into these descriptions and may pose some irregularities and may portray high roughness (Figure 7.16)

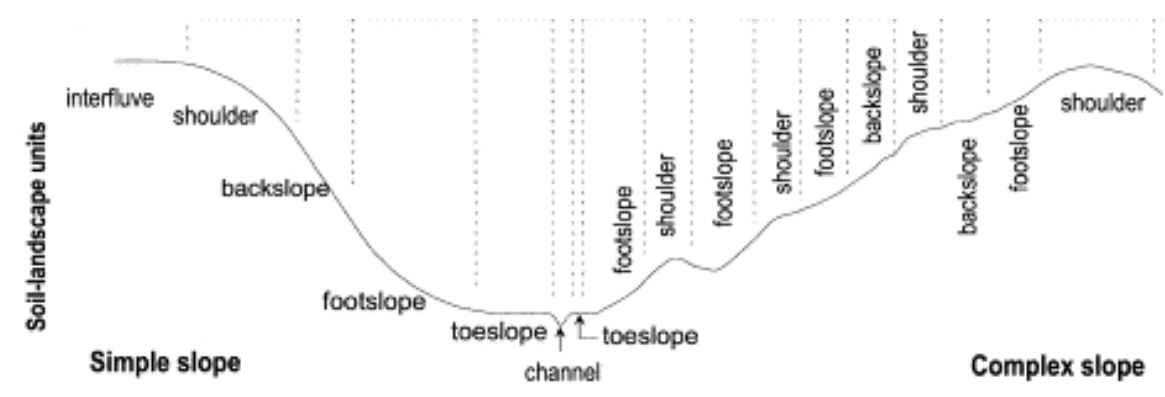

Figure 7.16: Simple slope vs. complex slope 


\section{CHAPTER 8}

\section{CONCLUSIONS}

Classification method produces landform classes that reveal general geomorphometry of terrain employing Object Based Image Analysis (OBIA) which is a fresh research topic in the context. Reasonable amount of consistency in different cases confirms that the results can be utilized as general or multi-purpose regarding some ambiguity that is already inherent in landforms.

Classes reveal general geomorphomery and they are suggested for use in general purposes or as basis for further analysis. Because, proposed classes may coincide or rather may have some conflict with other discipline perception worlds. However, method can be adjusted for specific purposes given expert-defined thresholds prior to classification.

'Semantics are the bridge between language and thought, and imply the meaning that is attached to real world objects and concepts. Therefore it is of great importance to construct a formal set of rules, and to successfully transport them into rules given relevant input information and set of rules described with stable criteria. These constraints were intended to be satisfied as straight forward and simple as possible with description of the rules. Anyway, classification with similar input and similar set of rules could end up with slightly different outputs than this study has yield. Because, an amount of unmanageable ambiguity is accompanied with classification based on semantics rather than descriptions pertaining to real entities or objects of natural kinds.

Classification employs same method and same set of parameters to cases to end up with general landform classes. Normalization of the DTMs enables use of same set of criteria for all the cases at different data ranges and as a result consistent classification outputs are obtained. However, for topography that portrays very smooth features e.g. a flat plain, a minimum threshold criteria for steepness or curvedness is required. 
Classification method brings together local geometry and geomorphometric context to end up with geomorphometric classes of general type. OBIA proved to be an efficient tool that it relates information from different levels within the framework of terrain context. However, method employs a loose coupling of form, position and terrain network and it can be improved into a more sophisticated and structured system that represents particular organization of terrain, however without regarding the fact that terrain may not always fit into particular organizations and may pose some irregularities and may portray for example high roughness. At those situations terrain position index that is employed for the classification to incorporate geomorphometric context should be prepared accordingly.

OBIA is rather utilized for classification purposes in this study; it enables gathering information content from different levels. However representation of scale at object domain in a multi-level hierarchy of landforms might be further exercised.

Window of perception (scale) has major influence on the outcomes of the classification of landforms. A coarser scale is likely to produce spatially generalized classes. However, not all the classes respond to change in scale in similar way. Some classes are replaced with others where some others are preserved at different scales of classification, those classes are considered to be scale-independendent; i.e. ridge.

Some of the fuzzy morphometric classes e.g. 'spur foot', 'hollow foot' pose poor classification statistics of classification stability and efficiency. They can be barely identified within the case areas. These classes can be reconsidered for improvement of their consistency with additional information, or with rule sets that put bias on these classes to identify them or optionally they can be embedded into a similar class, e.g. 'hollow foot' into 'hollow' or 'foot slope'.

Landforms are contiguous and the have rather fuzzy boundaries, they are semantically driven and have high scale dependencies where each observer tend to perceive them in different ways. Therefore standard accuracy assessment procedures widely applied on image classification results are not favorable to be adopted for landform classification. Rather a weighted index should be employed that represents matches and close matches as a weighted accuracy measure. 


\section{REFERENCES}

Addink, E. A., de Jong, S. M., Pebesma, E. J., 2007. The importance of scale in object based mapping of vegetation parameters with hyperspectral imagery. Photogrammetric Engineering and Remote Sensing, 73, 905-912.

Adedrian, A.O., Parcharidis, I., Poscolieri, M., Pavlopoulos, K., 2004. Computer-assisted discrimination of morphological units on north-central Crete (Greece) by applying multivariate statistics to local relief gradients. Geomorphology, 58, 357-370.

Arrell, K.E., Fisher, P.F., Tate, N.J., Bastin, L., 2007. A fuzzy c-means classification of elevation derivatives to extract the morphometric classification of landforms in Snowdonia, Wales. Computers and Geosciences, 33, 1366-1381.

Van Asselen, S., Seijmonsbergen, A.C., 2006. Expert-driven semi-automated geomorphological mapping for a mountainous area using a laser DTM. Geomorphology, 78, 309-320.

Baatz, M., Schäpe, A., 2000. Multiresolution Segmentation - an optimization approach for high quality multiscale image segmentation. In: Strobl, J., et al., eds. Angewandte Geographische Informationsverarbeitung XII. Wichmann,: Heidelberg, 12-23.

Bailey, R. G., 1996. Ecosystem Geography. Springer-Verlag, 216 p.

Band, L.E, Peterson, D.L., Running, S.W., Couglan, J.C., Lammers, R.B., Dungan, J., Nemani, R., 1991. Forest ecosystem processes at the watershed scale: basis for distributed simulation. Ecological Modelling, 56, 151-176.

Barringer, J.R.F., Hewitt, A.E., Lynn, I.H., Schmidt, J., 2008. National mapping of landform elements in support of S-Map, a New Zealand soils database. In: Zhou Q, Lees B, Tang G eds. Advances in Digital Terrain Analysis. Berlin, Springer, 443-458.

Behrens, T., 2003. Digitale Reliefanalyse als Basis von Boden-Landschafts-Modellen am Beispiel der Modellierung periglaziärer Lagen im Ostharz. Boden und Landschaft, 42, 189 p.

Behrens, T., Zhu, A-X., Schmidt, K., Scholten, T., 2009. Multi-scale digital terrain analysis and feature selection for digital soil mapping, Geoderma, 155, 175-185.

Bennett, D.A., Armstrong, M.P., 1996. An inductive knowledge-based approach to terrain feature extraction. Cartography and Geographic Information Systems, 23, 3-20.

Blaschke, T., Strobl, J., 2001. What is wrong with pixels? Some recent developments interfacing remote sensing and GIS. GISZeitschrift für Geoinformationssysteme, 6, 12-17.

Blaschke, T., Hay, G., 2001. Object-oriented image analysis and scale-space: theory and methods for modelling and evaluating multi-scale landscape structure. International Archives of Photogrammetry and Remote Sensing, 34, 22-29.

Blaschke, T., Strobl, J., 2003. Defining landscape units through integrated morphometric characteristics. In: Buhmann E., Ervin S. eds. Landscape Modelling: Digital Techniques for Landscape Architecture. Wichmann Verlag: Heidelberg, 104-113. 
Blaszczynski, J. S., 1997. Landform characterization with geographic information systems. Photogrammetric engineering \& remote sensing, 63, 183-191.

Bolongaro-Crevennaa, A., Torres-Rodriguez, V., Sorani, V, Frame, D., Ortiz, M.A., 2005. Geomorphometric analysis for characterizing landforms in Morelos State, Mexico. Geomorphology, 67, 407-422.

Brabyn, L. 1998: GIS analysis of macro landform. In: Proceedings, Spatial Information Research Centre's 10th Colloquium, New Zealand, University of Otago, 35-48.

Branting, L. K., Reiners, W. A., Wang H., 2000. Induction of Landtype Classification Rules form GIS Data. In: Proceedings of BESAI 2000 Workshop on Binding Environmental Sciences and Artificial Intelligence, August 21, 2000, Berlin, Germany.

Brown, D.G., Lusch, D.P., Duda, K.A., 1998. Supervised classification of types of glaciated landscapes using digital elevation data. Geomorphology, 21, 233-250.

Bull, W., 1975. Allometric change of landforms. Geological Society of America Bulletin 86, 14891498.

Burnett, C., Blaschke, T., 2003. A multi-scale segmentation/object relationship modelling methodology for landscape analysis. Ecological Modelling, 168, 233-249.

Burrough, P.A., 1986. Principles of Geographic Information Systems for Land Resource Assessment. Monographs on Soil and Resources Survey, vol. 12. Oxford Science Publications, New York, 193 p.

Burrough, P.A., 1989. Fuzzy mathematical methods for soil survey and land evaluation. Journal of Soil Science, 40, 477-492.

Burrough, P.A., van Gaans, P.F.M., MacMillan, R.A., 2000. High resolution landform classification using fuzzy-k means. Fuzzy Sets and Systems, 113, 37-52.

Burrough, P.A., Frank, A.U. eds., 1996. Geographic Objects with Indeterminate Boundaries. GIS data, vol. 2. London: Taylor \& Francis.

Burrough, P.A., McDonnell, R.A., 1998. Principles of Geographic Information Systems. Oxford, UK: Oxford University Press.

de Bruin, S., Stein, A., 1998. Soil-landscape modelling using fuzzy c-means clustering of attribute data derived from a Digital Elevation Model, DEM, Geoderma, 83, 17-33.

Canisius, F., Honda, K., Tokunaga, M., 2009. Updating geomorphic features of watersheds and their boundaries in hazardous areas using satellite synthetic aperture radar. International Journal of Remote Sensing, 30, 5919-5933.

Carrara, A., Cardinali, M., Detti, R., Guzzetti, F., Pasqui, V., Reichenbach, P., 1991. GIS techniques and statistical models in evaluating landslide hazard. Earth Surface Processes and Landforms, 16, 427-445.

Carrara, A., Pugliese-Carratelli, E., Merenda, L., 1977. Computer based data banks and statistical analysis for slope instability phenomena. Z. Geormorphol. N.F., 21, 187-222.

Cheng, T., Molenaar, M., 1999. Objects with fuzzy spatial extent. Photogrammetric Engineering and Remote Sensing, 63, 403-414.

Conacher, A.J., Dalrymple, J.B., 1977. The nine-unit landsurface model: an approach to pedogeomorphic research. Geoderma, 18, 1-154. 
Cayley, A., 1859. On contour and slope lines. The London, Edinburgh and Dublin Philosophical Magazine and Journal of Science Series 4, 18, 264-268.

Clark, M.L., Clark, D.B., Roberts, D.A., 2004. Small-footprint lidar estimation of sub-canopy elevation and tree height in a tropical rain forest landscape. Remote Sensing of Environment, 91, 6889.

Clarke, K.C., 1990. Analytical and Computer Cartography. Englewood Cliffs, NJ, Prentice Hall, p.290.

Cleland, D.T., Avers, P.E., McNab, W.H., Jensen, M.E., Bailey, R.G., King, T., Russell, W.E., 1997. National hierarchical framework of ecological units. In: Boyce, M.S., Haney, A. eds. Ecosystem Management. Yale University, New Haven, 181-200.

Coops, N.C., Gallant, J.C., Loughhead, A.N., Mackey, B.J., Ryan, P.J., Mullen, I.C., Austin, M.P., 1998. Developing and testing procedures to predict topographic position from Digital Elevation Models (DEMs) for species mapping (Phase 1). Report to Environment Australia, CSIRO Forestry and Forest Products, Canberra, Client Report No. 271.

Dalrymple, J., Long, R., Conacher, A., 1968. A hypothetical nine-unit land- surface model. Zeitschrift fur Geomorphologie, 12, 60-76.

Dehn, M., Gartner, H., Dikau, R., 2001. Principles of semantic modelling of landform structures. Computers and Geosciences, 27, 1005-1010.

Deng, Y.X., Wilson, J.P., Sheng, J., 2006. Effects of terrain attribute weights on landform classification. Earth Surf. Process. Landforms, 31, 1452-1462.

Deng, Y., 2007. New trends in digital terrain analysis: landform definition, representation, and classification. Progress in Physical Geography, 31, 405-419.

Deng, Y., Wilson, J.P., 2008. Multi-scale and multi-criteria mapping of mountain peaks as fuzzy entities. International Journal of Geographical Information Science, 22, 205-218.

Dikau, R. 1989, The application of a digital relief model to landform analysis. In: Raper, J.F., ed. Three Dimentional Applications in Geographic Information Sytems. London: Taylor and Francis, 5177.

Dikau, R., Brabb, E. E., Mark, R.M., 1991. Landform classification of New Mexico by computer. U.S. Geological Survey, Menlo Park, CA, Open-file rep., 91-634.

Dikau, R. 1992. Aspects of constructing a digital geomorphological base map. Geologisches Jahrbuch, A122, 357-370.

Dixon, T.H., 1991. An introduction to the global positioning system and some geological applications. Reviews of Geophysics, 29, 249-276.

Dong, Y., Tang, G., Zhang, T., 2008. A Systematic Classification Research of Topographic Descriptive Attribute in Digital Terrain Analysis. The International Archives of the Photogrammetry, Remote Sensing and Spatial Information Sciences, vol. XXXVII Part B2 Commission II, WG II/2 Spatial Reasoning, Analysis, and Data Mining, 357-362.

Drăguț, L., Blaschke, T., 2006. Automated classification of landform elements using object-based image analysis. Geomorphology, 81, 330-344.

Drăguț, L., Blaschke, T., 2009. , In: Zhou, Q., Lees, B., Tang, G. eds. Advances in Digital Terrain Analysis, Berlin Heidelberg, Springer-Verlag, 141-158. 
Dragut, L., Tiede, D., Levick, S.L., 2010. ESP: a tool for estimating the scale parameter for multiresolution image segmentation of remotely sensed data. International Journal of Geographical Information Science, 24(6), 859-871

Dunning, J.B., Danielson, B.J., Pulliam, H.R., 1992. Ecological processes that affect populations in complex landscapes. Oikos, 65,169-175.

Dymond, J.R., DeRose, R.C., Harmsworth, G.R., 1995. Automated mapping of land components from digital elevation data. Earth Surface Processes and Landforms, 20, 131-137.

ECOMAP, 1993. National hierarchical framework of ecological units. Unpublished administrative paper. Washington, DC. U.S. Department of Agriculture, Forest Service. 20p.

Eisank, C., Drăgut,, L., 2009. Multi-scale analysis of slope gradient using local variance graphs, GI Forum 2009, Salzburg, Austria

Evans, I.S., 1972. General geomorphometry, derivatives of altitude and descriptive statistics. In: Chorley R.J. ed. Spatial Analysis in Geomorphology. London: Harper \& Row, 17-90.

Evans, I.S., 1979. An integrated system of terrain analysis and slope mapping. Final report on grant DA-ERO-591-73-60040, University of Durham, England, 192 pp.

Evans, I.S., 1980. An integrated system of terrain analysis and slope mapping. Z. Geomorphol., Suppl. Bd., 36, 274-295.

Evans, I.S., 1998 .What do terrain statistics really mean. In: Lane, S., Richards, K., Chandler, J. eds. Landform Monitoring, Modelling and Analysis, John Wiley \& Sons, 119-138.

Evans, I.S., 1990. General Geomorphometry. In: Goudie, A.S., Anderson M., Burt T., Lewin, J., Richards, K., Whalley, B., Worsley, P. eds. Geomorphological Techniques. $2^{\text {nd }}$ edition. Unwin Hyman, London, 44-56.

Evans, I.S., 1980. An integrated system of terrain analysis and slope mapping. Zeitschrift für Geomorphologie, Supplementband, 36, 274-295.

Evans, I.S., 1972. General geomorphometry, derivatives of altitude, and descriptive statistics. In: Chorley, R.J., ed. Spatial Analysis in Geomorphology. Methuen, London, 17-90.

Evan, I.S., Cox, N.J., 1974. Geomorphometry and the operational definition of cirques. Area, 6, 150153.

Evans, I.S., Cox, N.J., 1999. Relations between land surface properties: altitude, slope and curvature. In: Hergarten, S., Neugebauer, H.J. eds. Process Modelling and Landform Evolution. Springer, Berlin, 13-45.

Etzelmüller, B., Sulebak, J.S., 2000. Developments in the use of digital elevation models in periglacial geomorphology and glaciology. Physische Geographie, 41, 35-58.

Eyton, J.R., 1991. Rate of Change Maps. Cartography and GIS's, 18, 87-103.

Fairfield, J., Leymarie, P., 1991. Drainage networks from grid digital elevation models. Water Resources Research, 27, 709-717.

Fels, J.E., 1995. Landscape Position and Classified Landtype Mapping for the Statewide DRASTIC Mapping Project. Raleigh, NC: North Carolina State University. Technical report to the North Carolina Department of Environment, Health, and Natural Resources, Division of Environmental Management. 
Fels, J.E., Matson, K.C., 1996. A cognitively based approach for hydro-geomorphic land classification using digital terrain models. In: Proceedings of the 3rd International Conference/Workshop on Integrating GIS and Environmental Modelling. Santa Fe, NM, January 2125, 1996.

Feitosa, R.Q., Costa, G.A.O.P., Cazes, T.B., Feijo, B., 2005. A genetic approach for the automatic adaptation of segmentation parameters. In: 1st International Conference on. Object Based Image Analysis, Salzburg, Austria

Fisher, P., Wood, J., Cheng, T., 2004. Where is Helvellyn? Fuzziness of multi-scale landscape morphometry, Trans. Inst. Br. Geogr, 29, 106-128.

Florinsky, I.V., 1998. Accuracy of local topographic variables derived from digital elevation models. International Journal of Geographical Information Science, 12, 47-61.

Florinsky, I.V., Eilers, R.G., Manning, G., Fuller, L.G., 2002. Prediction of soil properties by digital terrain modelling. Environmental Modelling \& Software, 17, 295-311.

Franklin, J., McCullough, P., Gray, C., 2000. Terrain variables used for predictive mapping of vegetation communities in Southern California. In: Wilson, J.P, Gallant, J.C., eds. Terrain Analysis: Principles and Applications. New York: Wiley, 331-354.

Fonesca, F.T., Egenhofer, M.J., Agouris, P., Camara, G., 2002. Using ontologies for integrated geographic information systems. Transactions in GIS, 6, 231-257.

Forman, R.T.T., Godron, M., 1986. Landscape Ecology. New York, Wiley, 620 p.

Fowler, R. J., Little, J.J., 1979. Automated extraction of irregular network digital terrain models. Computer Graphics, 13, 199 - 207.

Gale, S., 1972. Inexactness fuzzy sets and the foundation of behavioral geography. Geographical Analysis, 4, 337-349.

Gallant, J.C., Wilson, J.P., 2000. Primary Topographic Attributes. In: Gallant, J.C., Wilson, J.P. eds. Terrain Analysis: Principles and Applications. New York: Wiley, 51-85.

Gallant, A.L., Brown, D.D., Hoffer, R.M., 2005. Automated mapping of Hammond's landforms. IEEE Geoscience and Remote Sensing Letters, 2, 384-388.

Gauss, K.F., 1828. Disquisitiones generales circa superficies curvas. Commentationes Societatis Regiae Scientiarum Gottingensis, 6, 99-146.

Gessler, P. E., Moore, I. D., Mckenzie, N. J., Ryan, P. J., 1995. Soil-landscape modelling and spatial prediction of soil attibutes. International Journal of Geographical Information Systems, 9, 421-432.

Graff, L.H., Usery, E.L., 1993. Automated classification of generic terrain features in digital elevation models. Photogrammetric Engineering and Remote Sensing, 59, 1409-1417.

Gruber, S., Peckham, S., 2009. Land-Surface Parameters and Objects in Hydrology. In Hengl, T., Reuter, H.I., eds. Geomorphometry-Concepts, Software, Applications. Developments in Soil Science, vol. 33, Amsterdam, Elsevier, 171-194.

Hall, S.T., 2008. Topographic analysis and Predictive Modelling using Geographic Information Systems, Thesis (PhD), the Graduate School of Clemson University, USA.

Hall, O., Hay, G.J., Bouchard, A., Marceau, D.J., 2004. Detecting dominant landscape objects through multiple scales: an integration of object-specific methods and watershed segmentation. Landscape Ecology, 19, 59-76. 
Hammer, R.D., 1997. Space and time in the soil landscape: the ill-defined ecological universe. In: Peterson, D.L., Parker, V.T., eds. Ecological Scale: Theory and Applications. Palisades, NY: Columbia University Press.

Hammond, E.H., 1964. Analysis of properties in land form geography: An application to broad-scale land form mapping. Ann. Assoc. Amer. Geogr., 54, 11-19.

Hammond, E.H., 1965. What is a landform? Some further comments. The Professional Geographer, $17,12-13$

Hammond, E. H., 1970. Classes of land-surface form, The National Atlas of the United States of America. Reston, VA: U.S. Geological Survey, 62-64.

Hanssen, R.F., 2001, Radar Interferometry-Data Interpretation and Error Analysis, Dordrecht/Boston/London, Kluwer, 308 p.

Haralick, R., Shanmugan, K., Dinstein, I., 1973. Textural features for image classification. IEEE Trans. Systems Man Cybernetics, 3, 610-621.

Hay, G.J., Castilla, G., 2006. Object-based image analysis: Strengths, weaknesses, opportunities and threats (SWOT). In: Proceedings OBIA 2006, Commission VI, WG VI/4, Calgary, CA.

Hay, G.J., Castilla, G., 2008. Geographic Object-Based Image Analysis (GEOBIA): A new name for a new discipline. In: Blaschke, T., Lang, S., Hay, G., eds. Object-Based Image Analysis. Springer, Heidelberg, Berlin, New York, 93-112.

Heuvelink, G.B.M., Burrough, P.A., 1993. Error propagation in cartographic modelling using boolean logic and continuous classification. Int. J. Geographical Information Systems, 7, 231-246.

Hirano, A., Welch, R., Lang, H., 2003. Mapping from ASTER stereo image data: DEM validation and accuracy assessment. ISPRS Journal of Photogrammetry and Remote Sensing, 57, 356-370.

Hoffmann, A., van der Vegt, J.W., 2001. New sensor systems and new classification methods: laserand digital camera-data meet object oriented strategies. GeoBIT/GIS 6, 16-23.

Horton, R.E., 1945. Erosional development of streams and their drainage basins; hydrophysical approach to quantitative morphology. Geol. Soc. Amer. Bull., 56, 275-370.

Hosokawa, M., Zama, Sh., Hoshi, T., 2002. Supervised landform classification method using neural network and its application to estimation of seismic ground motion. Journal of Structural and Construction Engineering, 555, 69-76.

Hugget,R.J., 1975. Soil landscape systems: A model of soil Genesis. Geoderma, 13, 1-22.

Hurlbert, S.H., 1984. Pseudoreplication and the design of ecological field experiments. Ecol. Monogr, 54, 187-211.

Hutchinson, M.F., 1989. A new procedure for gridding elevation and stream line data with automatic removal of spurious pits. Journal of Hydrology, 106, 211-232.

Hutchinson, M.F., Gallant, J.C., 2000. Digital Elevation Models and Representation of Terrain Shape. In Gallant J.C., Wilson J.P. eds. Terrain Analysis: Principles and Applications. New York: Wiley, $29-50$.

Irvin, B.J., Ventura, S.J., Slater, B.K., 1997. Fuzzy and isodata classification of landform elements from digital terrain data in Pleasant Valley, Wisconsin. Geoderma, 77, 137-154. 
Jackendoff, R., 1983. Semantics and Cognition, Cambridge, MA: MIT Press.

Jones, K.H., 1998. Comparison of algorithms used to compute hillslope as a property of the DEM. Computers \& Geosciences, 24, 315-323.

Klingseisen, B., 2004. GIS based generation of topographic attributes for landform classification, Thesis (PhD), Curtin University of Technology, Perth, Western Australia.

Kühni, A., Pfiffner, O.A., 2001. The relief of the Swiss Alps and adjacent areas and its relation to lithology and structure- topographic analysis from 250-M DEM. Geomorphology, 41, 285-307.

Li, Z., 2008. Multi-scale terrain modelling and analysis. In Zhou, Q., Lees, B., Tang, G.A., eds. Advances in Digital Terrain Analysis. Springer, Berlin, Heidelberg, 59-83.

Li, Z., Zhu, Q., Gold, C., 2005. Digital Terrain Modelling: Principles and Methodology. CRC Press, Boca Raton, FL, 319 p.

Longley, P.A., Goodchild, M.F., Maguir,e D.J., Rhind, D.W. 2001. Geographic Information Systems and Science. Wiley, pp. 144-147.

Mackaness, W., Beard, K., 1993. Use of graph theory to support map generalisation, Cartography and Geographical Information Systems, 20, 210-221.

Mackay, D.S., Robinson, V.B., Band L.E., 1992. Classification of Higher Order Topographic Objects on Digital Terrain Data. Computers, Environment and Urban Systems, 16, 473-496.

MacMillan, R.A., Pettapiece, W.W., 2000. Alberta Landforms: Quantitative morphometric descriptions and classification of typical Alberta landforms, Research Branch, Agriculture and AgriFood Canada, Semiarid Prairie Agricultural Research Centre, Swift Current, SK, Technical Bulletin No. 2000-2E.

Macmillan R., Pettapiece W., Nolan, S., Goddard, T., 2000. A generic procedure automatically segmenting landforms into landform elements using DEMs, heuristic rules and fuzzy logic. Fuzzy sets and Systems, 113, 81-109.

Macmillan, R.A., Keithjones, R., Mcnabb, D.H., 2004. Defining a hierarchy of spatial entities for environmental analysis and modelling using digital elevation models (DEMs). Computers, Environment and Urban Systems, 28, 175-200.

MacMillan, R.A., Moon, D.E., Coupé, R.A., 2007. Automated predictive ecological mapping in a Forest Region of B.C., Canada, 2001-2005. Geoderma, 140, 353-373.

MacMillan, R.A., Shary, P.A., 2009. Landforms and Landform Elements in Geomorphometry.

In: Hengl, T., Reuter, H.I., eds. Geomorphometry-Concepts, Software, Applications. Developments in Soil Science, vol. 33, Amsterdam, Elsevier, 227-254.

Mallinis, G., Koutsias, N., Tsakiri-Strati M., Karteris, M., 2008. Object-based classification using Quickbird imagery for delineating forest vegetation polygons in a Mediterranean test site. ISPRS Journal of Photogrammetry and Remote Sensing, 63, 237-250.

Mark, D.M., 1975. Computer analysis of topography: a comparison of terrain storage methods, Geografiska Annaler, 5, 179-188.

Mark, D.M., Turk, A.G., 2003. Landscape categories in Yindjibarndi: Ontology, environment, and language. Lecture Notes in Computer Science, 2825, 28-45. 
Martin, Y., 2000. Modelling hillslope evolution: linear and nonlinear transport relations. Geomorphology, 34, 1-21.

Martin, Y., Church, M., 2004. Numerical modelling of landscape evolution: geomorphological perspectives. Progress in Physical Geography, 28, 317-339.

Matternicht, G., 1999,. Change detection assessment using fuzzy sets and remotely sensed data: an application of topographic map revision,. ISPRS Journal of Photogrammetry and Remote Sensing. $54,221-233$.

Maune, D.F., ed., 2001. Digital Elevation Model Technologies and Applications, the DEM Users Manual, Bethesda, MD, American Society for Photogrammetry \& Remote Sensing, 540 p.

Maxwell, J.C., 1870. On contour lines and measurements of heights. The London, Edinburgh and Dublin Philosophical Magazine and Journal of Science, 40, 421-427.

McBratney, A.B., de Gruijter, J.J., Brus, D.J., 1992. Spatial prediction and mapping of continuous soil classes. Geoderma, 54, 39-64.

McKenzie, N.J., Ryan, P.J., 1999. Spatial prediction of soil properties using environmental correlation. Geoderma, 89, 67-94.

McKenzie, N.J., Gessler, P.E., Ryan, P.J., O’Connell, D.A., 2000. The Role of terrain analysis in soil mapping. In: Wilson, J.P., Gallant, J.C. eds. Terrain Analysis, Principles and Applications. John Wiley \& Sons, New York, USA, 245-265.

Meijerink, A.M.J., 1988. Data acquisition and data capture through terrain mapping units. ITC Journal, 1, 23-44.

Meinel, G., Neubert, M., 2004. A comparison of segmentation programs for high resolution remote sensing data, In: Proceedings of the ISRPS 2004 Annual Conference, Istanbul, Turkey, 19 - 23.

Miller, J.C., 1953. A quantitative geomorphic study of drainage basin characteristics in the Cinch Mountain Area, Virginia and Tennessee, Tech. Rep. no.:3.

Milne, G., 1935. Some suggested units of classification and mapping, particularly for East African soils. Soil Res., 4, 183-198.

Milne G., 1936. A provisional soil map of East Africa, Amani Memoirs No. 28. Eastern African Agricultural Research Station, Tanganyika Territory.

Mitas, L., Mitášová, H., 1999. Spatial interpolation. In: Longley, P., Goodchild, M.F., Maguire, D.J., Rhind, D.W. eds. Geographical Information Systems: Principles, Techniques, Management and Applications, vol. 1. Wiley, 481-492.

Montello, D. R., 2001. Scale in geography. In Smelser N. J., Baltes P. B. eds. International Encyclopedia of the Social \& Behavioral Sciences. Oxford: Pergamon Press, 13501-13504.

Moore, I.D., Gessler, P.E., Nielsen, G.A., Peterson, G.A., 1993. Soil attribute prediction using terrain analysis. Soil Science Society of America Journal, 57, 443-452.

Moore, I.D., Grayson, R.B., Ladson, A.R., 1991. Digital terrain modelling: a review of hydrological, geomorphological, and biological applications. Hydrological Processes, 5, 3-30.

Moore, I.D., Lewis, A., Gallant, J.C., 1993. Terrain attributes: Estimation methods and scale effects. In: Jakeman, A.J., Beck, M.B., McAleer, M.J. eds. Modelling Change in Environmental Systems. John Wiley, Chichester, 189-214. 
Nelson, A., Reuter, H.I., Gessler, P., 2009. Geomorphometry: A Brief Guide. In Hengl, T. and Reuter, H.I., editors, Geomorphometry-Concepts, Software, Applications. Developments in Soil Science, vol. 33, Amsterdam: Elsevier, 65-85.

O’Callaghan, J.F., Mark, D.M., 1984. The extraction of drainage networks from digital elevation data, Computer Vision, Graphics and Image Processing, 28, 323-344.

Park, S.J., van de Giesen, N., 2004. Soil-landscape delineation to define spatial sampling domains for hillslope hydrology, Journal of Hydrology, 295, 28-46.

Penck, A., 1894. Morphologie der Erdoberflache. Engelhorn-Verlag, Stuttgart, Germany, vol. 1, 471 p.

Pennock, D.J., 2003. Terrain attributes, landform segmentation, and soil redistribution. Soil \& Tillage Research, 69, 15-26.

Pennock, D.J., Zebarth, B.J., De Jong, E., 1987. Landform classification and soil distribution in Hummocky terrain, Saskatchewan, Canada. Geoderma, 40, 297-315.

Peucker, T.K., Douglas, D.H., 1974. Detection of surface specific points by local parallel processing of discrete terrain elevation data. Computer Graphics and Image Processing, 4,375-387.

Pfeffe, K., Pebesma, E.J., Burrough, P.A., 2003. Mapping alpine vegetation using vegetation observation and topographic attributes. Landscape Ecology, 18, 759-76.

Pfaltz, J., 1976. Surface Networks. Geographical Analysis, 8, 77-93.

Phillips, J. D., 2005. Weathering instability and landscape evolution. Geomorphology, 67, 255-272.

Pike, R.J., 1988. The geometric signature: quantifying landslide terrain types from digital elevation models. Mathematical Geology, 20, 491-511.

Pike, R.J., 2002. A bibliography of terrain modelling (geomorphometry), the quantitative representation of topography - supplement 4.0., Open-File Rep. No. 02-465. U.S. Geological Survey, Denver, 116 p.

Pike, R.J., Evans, I.S., Hengl, T., 2009. Geomorphometry: A Brief Guide. In Hengl, T., Reuter H.I., eds. Geomorphometry-Concepts, Software, Applications. Developments in Soil Science, vol. 33, Amsterdam: Elsevier, 3-30.

Pike, R.J., Wilson, S.E., 1971. Elevation-Relief Ratio, Hypsometric Integral, and Geomorphic AreaAltitude Analysis. Geol Soc Amer Bull, 82, 1079-1084.

Plewe, B., 1997. A representation-oriented taxonomy of gradation. In Hirtle, S.C., Frank, A.U., eds. Spatial information theory: a theoretical basis for GIS, Lecture Notes in Computer Sciences 1329, Berlin: Spring- Verlag, 121-136.

Prima, A., Echigo, A., Yokoyama, R., Yoshida, T., 2006. Supervised landform classification of Northeast Honshu from DEM-derived thematic maps. Geomorphology, 78, 373-386.

Prosser, I.P., Abernethy, B., 1996. Predicting the topographic limits to a gully network using a digital terrain model and process thresholds. Water Resources Research, 32, 2289-2298.

Qin, C.Z., Zhu, X., Shi, X., Li, B.L., Pei, T., Zhou, C.H., 2009. Quantification of spatial gradation of slope positions. Geomorphology, 110, 152-161.

Rana, S., 2004. Topological Data Structures for Surfaces: An Introduction for Geographical Information Science., Wiley, New York, 214 p. 
Rana, S., 2006. Use of Plan Curvature Variations for the Identification of Ridges and Channels on DEM. In: Riedl, A., Kainz, W., Elmes, G.A.eds. Progress in Spatial Data Handling, 789-804.

Rana, S., Wood J.D., 2000. Weighted and metric surface networks, Working Paper 25, Center for Advanced Spatial Analysis, CASA, UCL

Rasemann, S., Schmidt, J., Schrott, L., Dikau, R., 2004. Geomorphometry in mountain terrain. In: Bishop, M.P., Shroder, J.F. eds. GIS \& Mountain Geomorphology. Springer, Berlin, 101-145.

Reuter, H.I., Wendroth O., Kersebaum, K.C., 2006. Optimisation of relief classification for different levels of generalisation. Geomorphology, 77, 79-89.

Richter, H., 1962. Eine neue Methode der groBmal3stabigen Kartierung des Reliefs. Petermanns Geographische Mitteilungen, 106, 309-312.

Robinson, V.B., 1988. Some implications of fuzzy set theory applied to geographic databases. Computers, Environment and Urban Systems, 12, 89-97.

Romstad, B., 2001. Improving relief classification with contextual merging. In: Bjorke, J.T., Havard, T. eds. Proceedings of the 8th Scandinavian Research Conference on Geographical Information Science. ScanGIS'2001, 25th-27th June 2001. As, Norway, 3-14.

Rosch, E.H., 1973. Natural categories. Cognitive Psychology, 4, 328-350.

Ruhe, R.V., 1975. Geomorphology-Geomorphic Processes and Surficial Geology. Houghton-Miflin, Boston, 246 p.

Ruhe, R.V., Walker, P.H., 1968. Hillslope models and soil formation II: open systems. In: Proceedings of 9th Congress of the International Soil Science Society, vol. 4. International Soil Science Society, Adelaide, Australia, 551-560.

Sainsbury, R.M. 1989. What is a vague object? Analysis, 49, 99-103.

Sankey, H., 1997. Induction and natural kinds. Principia, 1, 239-254.

Shapiro, L.G. and Stockman G.C., 2001. Computer Vision, New Jersey, Prentice-Hall, 279-325.

Schloss M., 1966. Quantifying terrain roughness on lunar and planetary surfaces. Journal of Spacecraft and Rockets, 3, 2, 283-285.

Schmidt, J., Andrew, R., 2005. Multi - scalelandform characterization. Area, 37, 341-350.

Schmidt, J., Evans, I.S., Brinkmann, J., 2003. Comparison of polynomial models for land surface curvature calculation. International Journal of Geographical Information Science, 17, 797- 814.

Schmidt, J., Hennrich, K., Dikau, R., 2000. Scales and similarities in runoff processes with respect to geomorphometry. Hydrological Processes, 14, 1963-1979.

Schmidt, J., Hewitt, A., 2004. Fuzzy land element classification from DTMs based on Geometry and terrain position. Geoderma, 121, 243-256.

Schmidt, McGibbon, 1993. Landform mapping, analysis and classification using Digital Terrain Models, Thesis (PhD), University of Alberta, p.212

Schneider, B., 2003. Surface Networks: Extension of the Topology and Extraction from Bilinear Surface Patches, Proceedings of the 7th International Conference on GeoComputation, University of Southampton, United Kingdom. 
Shary, P., 1995. Land surface in gravity points classification by a complete system of curvatures. Mathematical Geology, 27, 373-390.

Shary, P.A., Sharaya, L.S., Mitusov, A.V., 2005. The problem of scale-specific and scale-free approaches in geomorphometry. Geografia Fisica e Dinamica Quaternaria, 28, 81-101.

Shi, X., Zhu, A.-X., Wang, R.-X., 2005. Fuzzy representation of special terrain features using a similarity-based approach. In: Cobb, M., Petry, F., Robinson, V., eds. Fuzzy Modelling with Spatial Information for Geographic Problems. Springer-Verlag, New York, 233-251.

Sinha, G., 2008. Delineation, Characterization and classification of Topographic Eminences, Thesis $(\mathrm{PhD})$, University of New York at Buffalo.

Skidmore, A.K., 1990. Terrain position as mapped from a gridded digital elevation model. International Journal of Geographical information Systems, 4, 33-49.

Skidmore, A.K., Watford, F., Luckananurug, P., Ryan, P.J., 1996. An operational GIS expert system for mapping forest soils. Photogrammetric Engineering and Remote Sensing, 62, 501-511.

Smith B, 1995. The structures of the commonsense world. Acta Philosophica Fennica, 58, 290-317.

Smith, B., Mark, D.M., 2003. Do mountains exist? Towards an ontology of landforms. Environment and Planning B: Planning and Design, 30, 411-427.

Sowa, J.F., 2000. Knowledge Representation: Logical, Philosophical and Computational Foundations, Brooks Cole Publishing Co., Pacific Grove, CA, 560 p.

Speight, J.G., 1974. A parametric approach to landform regions. In: Brown, E.H., Waters, R.S., eds. Progress in Geomorphology, London: Alden Press, 213-230.

Speight, J.G., 1990. Landform. In: McDonald, et al. eds., Australian Soil and Land Survey Field Handbook, $2^{\text {nd }}$ ed. Inkata Press, Melbourne, 9-57.

Stepinski, T.F., Ghosh S., Vilalta, R., 2006. Automatic recognition of landforms on Mars using terrain segmentation and classification. In: Lavra c, N., Todorovski, L., Jantke, K.P. eds. DS 2006, LNAI 4265, Berlin Heidelberg: Springer-Verlag, 255-266.

Strahler, A.N., 1952. Hypsometric (area-altitude) analysis of erosional topography. Geological Society of America Bulletin, 63, 117-1141.

Strahler, A. N. 1964. Quantitative geomorphology of drainage basins and channel networks. In: Chow, V. T., ed. Handbook of Applied Hydrology, McGraw-Hill, Reading, MA., 439-476.

Strobl, J., 2007. Segmentation-based Terrain Classification, In: Zhou Q., Lees, B., Tang, G., eds. Advances in Digital Terrain Analysis, Berlin Heidelberg: Springer-Verlag, 125-141.

Terunuma T., Nishida, K., Amada, T., Mizuyama, T., Sato, I. Urai, M., 2005, Detection of traces of pyroclastic flows and lahars with satellite synthetic aperture radars. International Journal of Remote Sensing, 26, 1927-1942.

Tobler, W.R., 1970. Selected Computer Programs. Department of Geography, University of Michigan, Ann Arbor, MI.

Troeh, F.R., 1965. Landform equations fitted to contour maps. American Journal of Science, 263, 616-627. 
Unwin, D.J., 1975. Introduction to trend surface analysis, Concepts and Techniques in Modern Geography (CATMOG), Norwich:Geobook,. 40 p.

Van Westen, C., 1993. Gissiz, Training package for Geographic Information Systems in Slope Instability Zonation. ITC Publication No. 15. ITC, Enschede, The Netherlands, 245 p.

Verhagen, J., Verburg, P., Sybesma, M., Bouma, J. et al., 1995. Terrain modelling as a basis for optimal agroecological land management using dynamic simulation. In: Robert, P.C., ed. SiteSpecific Management for Agricultural Systems. ASA, CSSA, and SSSA, Madison, WI, 229-250.

Walker, J., Blaschke, T., 2008. Object-based landcover classification for the Phoenix metropolitan area: optimization vs. transportability. International Journal of Remote Sensing, 29, 2021-2040.

Watson, D.F. 1992: Contouring: a guide to the analysis and display of spatial data. Computer Methods in the Geosciences, vol. 10. Oxford: Pergamon Press.

Weibel, R., Heller, M., 1991. Digital terrain modelling. In: Maguire, D.J., Goodchild, M.F., Rhind, D.W. eds. Geographical Information Systems, vol. 1., Longman, London, 269-297.

Werner, C., 1988. Formal analysis of ridge and channel patterns in maturely eroded terrain. Annals of the Association of American Geographers, 78, 253-270.

Werner, C., 1994. Explorations into the formal structure of drainage basins. Earth Surface Processes and Landforms, 19, 747-762.

Whittow, J., 1984. Dictionary of Physical Geography. Penguin Reference Series, Middlesex, USA, $591 \mathrm{p}$.

Williamson, T., 1994. Vagueness, London, Routledge, NY, USA, 306 p.

Wilson, J.P., Gallant, J.C., 2000. Digital Terrain Analysis. In: Wilson, J.P., Gallant J.C., eds. Terrain Analysis: Principles and Applications. New York: Wiley, 1-29.

Wolf, G.W., 1988. Weighted Surface Networks and their Application to Cartographic Generalisation. In: Barth, W., ed. Visualisierungstechniken und Algorithmen, Springer Verlag, Berlin, 199-212.

Wolf, G.W., 1991. A FORTRAN subroutine for cartographic generalization. Computers and Geosciences, 17, 1359 - 1381 .

Wood, A., 1942. The development of hillside slopes. Proceedings of the Geological Association, $53,128-140$

Wood, J.D., 1996. Scale-based characterization of Digital Elevation Models. In: Parker, D., ed. Innovations in GIS 3, Taylor \& Francis, London,UK, 163-174.

Wood, J.D., 1996. The geomorphological characterization of Digital Elevation Models, Thesis (PhD), University of Leicester.

Wood, J.D., 2000. Constructing Weighted Surface Networks for the Representation and Analysis of Surface Topology. In: Proceedings 5th International Conference on GeoComputation, Chatham, UK, September 23-25th, 2000

Wood, J.D., 1998. Modelling the Continuity of Surface Form Using Digital Elevation Models. In: Proceedings of the 8th International Symposium on Spatial Data Handling, 725-736.

Wood, J.D., 1999, Visualization of Scale Dependencies in Surface Models. Presented to the International Cartographic Association Annual Conference, Ottawa. 
Wood, J.D., Rana, S., 2000. Constructing Weighted Surface Networks for the Representation and Analysis of Surface Topology. In: Proceedings 5th International Conference on GeoComputation, Chatham, UK, $264 \mathrm{p}$.

Wood, J.D., 2009. Geomorphometry in LandSerf In: Hengl, T., Reuter, H.I., eds. GeomorphometryConcepts, Software, Applications, Developments in Soil Science, vol. 33, Elsevier, Amsterdam, 333349.

Wood, J.D., 1996-2010. Landserf (Version 2.3) [Computer software]. California, USA, IEEE Visualization 2007.

Woodcock, C., Harward, V. J., 1992. Nested-hierarchical scene models and image segmentation. International Journal of Remote Sensing, 13, 3167-3187.

Woodcock, C., Strahler, A., 1987. The factor of scale in remote sensing. Remote Sensing of Environment, 21, 311-332.

Young, A., 1972. Slopes (Clayton, K.M., ed.).Edinburgh, Scotland, Oliver and Boyd Ltd., 288 p.

Young, M. 1978. Terrain Analysis: program Documentation. Report on Grant DA-ERO-591-7360040 Statistical Characterization of Altitude matrices by computer, European Research office, U.S. Army, I.S. evans, Principal Investigator, University of Durham, England.

Zadeh, L., 1965. Fuzzy sets. Information and Control, 8, 338-353.

Zhu, A.X., Burt, J.E., Smith, M., Wang, R., Gao, J., 2008. The Impact of Neighborhood Size on Terrain Derivatives and Digital Soil Mapping. In: Zhou, Q., Lees, B., Tang, G., eds. Advances in Digital Terrain Analysis, Berlin, Heidelberg, Springer-Verlag, 333-349.

Zuidam, R.A., 1986. Aerial Photo-Interpretation in Terrain Analysis and Geomorphologic Mapping. International Institute for Aerospace Survey and Earth Sciences, Netherlands:Smits Publishers, 422 p.

Zevenbergen, L.W., Thorne, C.R., 1987. Quantitative analysis of land surface topography. Earth Surface Processes and Landforms, 12, 47-56.

Wilson, J.P., Gallant, J.C., 1998. Terrain-based approaches to environmental resource evaluation. In: Lane, S., Richards, K., Chandler, J., eds. Landform Monitoring, Modelling and Analysis. Wiley, Chichester, 219-240.

\section{Web Sources}

ArcGIS Desktop Help 9.3, http://webhelp.esri.com/arcgisdesktop/9.3 (last accessed on February 2010)

Definiens Developer 7, ReferenceBook, http://www.ecognition.cc/download/ReferenceBook.pdf (last accessed on February 2010).

Definiens Partner Evolve Program, Definiens http://www.definiens.com/academic-centers-ofexcellence_129_20_22.html, (last accessed on February 2010).

Jenness, J., 2006. Topographic Position Index (tpi_jen.avx) extension for ArcView 3.x, v. 1.3a. Jenness Enterprises, http://www.jennessent.com/arcview/tpi.htm, (last accessed on February 2010).

Shuttle Radar Topography Mission, NASA, Jet Propulsion Laboratory, California Institute of Technology, http://www2.jpl.nasa.gov/srtm/coverage.html, (last accessed on March 2009). 
Landserf, http://www.soi.city.ac.uk/ jwo/landserf, (last accessed on January 2010).

Hatfield, D.C., 1999. TopoTools- A Collection of Topographic Modelling Tools for ArcINFO,http://proceedings.esri.com/library/userconf/proc00/professional/papers/PAP560/p560.htm, (last accessed in 2009).

USGS, DEM error, http://rmmcweb.cr.usgs.gov/ (last accessed on March 2009). 


\section{APPENDIX A}

\section{RMS SLOPE CALCULATION SCRIPT}

\#calculates slope for a given raster object \#script by Deniz Gerçek \#generated on 04.01 .2009

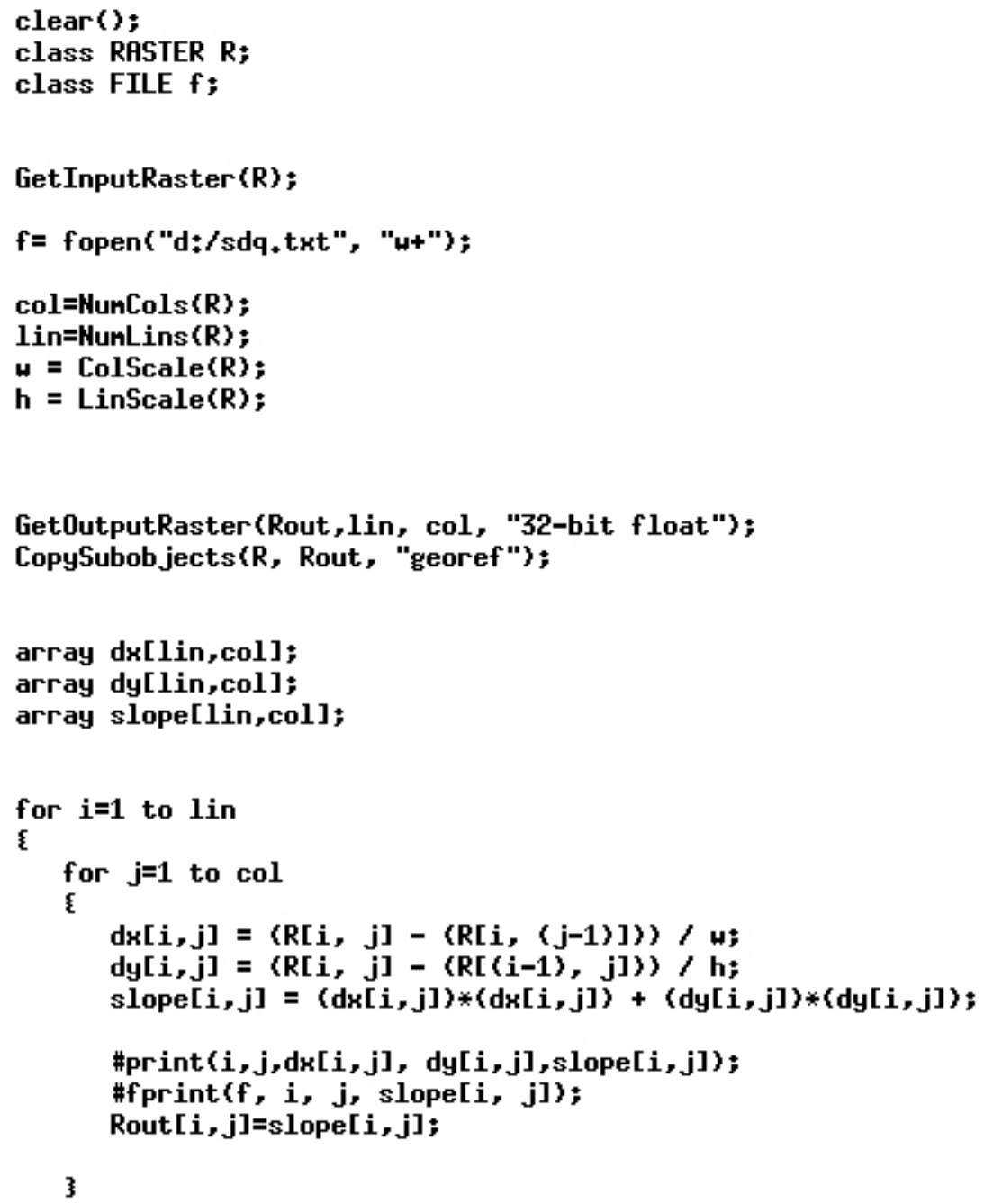




\section{APPENDIX B}

\section{RMS ERROR OF DEMs}

Table B.1: Elevation values of sample Ground Control points compared to corresponding grid elevations at varying resolutions of DEMs for case 1

\begin{tabular}{|r|r|r|r|r|r|r|}
\hline Case1 & $\begin{array}{l}\text { Control } \\
\text { point } \\
\text { elevation }\end{array}$ & DEM10x10 & DEM15x15 & DEM20x20 & DEM25x25 & DEM30x30 \\
\hline 1 & 634 & 632.51 & 632.45 & 626.34 & 630.43 & 626.43 \\
\hline 2 & 422 & 420.09 & 423.62 & 423.96 & 418.14 & 415.32 \\
\hline 3 & 1171 & 1170 & 1170.05 & 1170.39 & 1166.64 & 1169.15 \\
\hline 4 & 1103 & 1106.68 & 1100.95 & 1105.44 & 1100.36 & 1101.08 \\
\hline 5 & 657 & 657.25 & 654.89 & 653.98 & 653.29 & 648.96 \\
\hline 6 & 1253 & 1253.924 & 1252.37 & 1249.98 & 1253.34 & 1248.71 \\
\hline 7 & 1811 & 1812.23 & 1812.89 & 1812.28 & 1809.88 & 1809.47 \\
\hline 8 & 1531 & 1530.63 & 1530.27 & 1530.24 & 1530.23 & 1530.19 \\
\hline 9 & 1888 & 1887.77 & 1887.58 & 1886.02 & 1885.8 & 1883.98 \\
\hline 10 & 1244 & 1246.54 & 1247.48 & 1244.54 & 1243.89 & 1237.54 \\
\hline RMSeror & & 6.307692 & 5.3076923 & 18.538462 & 19.076923 & 30.53846 \\
\hline
\end{tabular}

Table B.2: Elevation values of sample Ground Control points compared to corresponding grid elevations at varying resolutions of DEMs for case 2

\begin{tabular}{|r|r|r|r|r|r|r|}
\hline Case2 & $\begin{array}{l}\text { Control } \\
\text { point } \\
\text { elevations }\end{array}$ & DEM10x10 & DEM15x15 & DEM20x20 & DEM25x25 & DEM30x30 \\
\hline 1 & 1127 & 1126.94 & 1126.39 & 1126.28 & 1126.5 & 1126.35 \\
\hline 2 & 931 & 929.6 & 931.55 & 932.33 & 933.8 & 932.52 \\
\hline 3 & 1576 & 1576.14 & 1575.68 & 1576.45 & 1576.5 & 1576.14 \\
\hline 4 & 1127 & 1126.94 & 1126.397 & 1126.28 & 1126.5 & 1126.35 \\
\hline 5 & 942 & 945.26 & 943.94 & 945.08 & 943.99 & 944.39 \\
\hline 6 & 657 & 657.32 & 657.62 & 657.47 & 657.44 & 657.64 \\
\hline 7 & 1475 & 1470.79 & 1475.58 & 1474.69 & 1477.05 & 1476.07 \\
\hline 8 & 713 & 711.16 & 712.79 & 713.53 & 712.68 & 711.89 \\
\hline RMSerror & & 2.6020385 & 0.4360853 & 1.007115 & 1.311431 & 0.897977 \\
\hline
\end{tabular}




\section{APPENDIX C}

\section{LANDSERF CHARACTERISTIC SCALE BY WOOD (2009)}

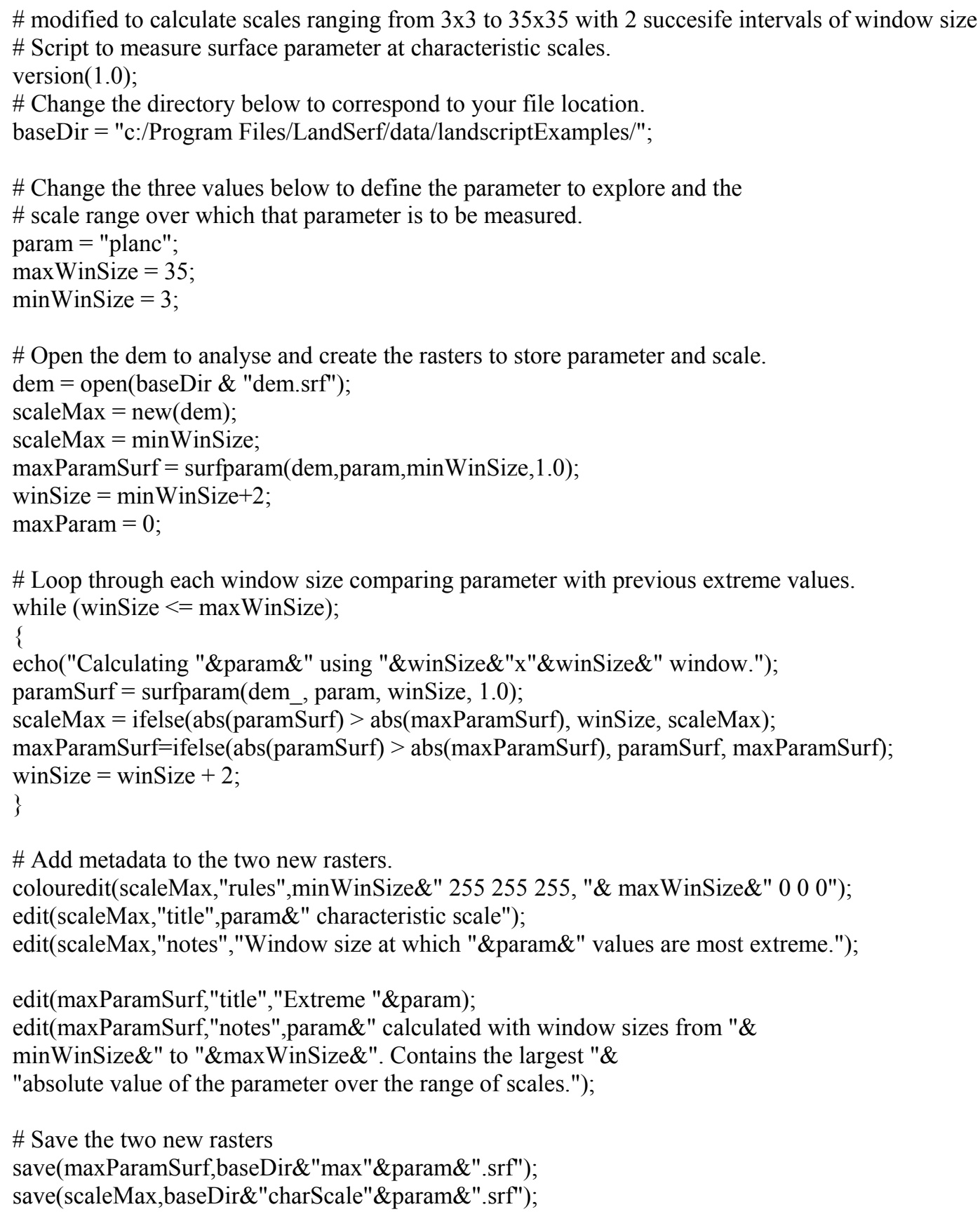




\section{APPENDIX D}

\section{ESP RESULTS: RATE OF CHANGE (ROC) OF LV}

Table D: ESP results for 'case1' and 'case2'

\begin{tabular}{|c|c|c|c|c|c|c|c|c|}
\hline \multirow[b]{2}{*}{ Scale } & \multicolumn{2}{|l|}{ caselfine } & \multirow{2}{*}{\multicolumn{2}{|c|}{$\begin{array}{l}\text { case } 1 \\
\text { stddev/ } \\
\text { mean }\end{array}$}} & \multirow{2}{*}{\multicolumn{2}{|c|}{$\begin{array}{l}\text { case 1 coarse } \\
\text { stddev/ } \\
\text { mean }\end{array}$}} & \multirow{2}{*}{\multicolumn{2}{|c|}{$\begin{array}{l}\text { case } 2 \\
\text { stddev/ } \\
\text { mean }\end{array}$}} \\
\hline & $\begin{array}{l}\text { stddev/ } \\
\text { mean }\end{array}$ & $\mathrm{ROC}$ & & & & & & \\
\hline 1 & 0.138503 & 69.1708 & 0.088465 & 73.47148 & 0.050232 & 82.48802 & 0.050726 & 72.27937 \\
\hline 2 & 0.234306 & 23.86219 & 0.153461 & 30.3434 & 0.091667 & 37.30437 & 0.087391 & 31.55382 \\
\hline 3 & 0.290217 & 13.94545 & 0.200026 & 18.21776 & 0.125863 & 18.60702 & 0.114966 & 19.96355 \\
\hline 4 & 0.330689 & 11.23259 & 0.236467 & 11.85452 & 0.149283 & 16.92782 & 0.137917 & 13.92386 \\
\hline 5 & 0.367834 & 9.144519 & 0.264499 & 8.958878 & 0.174553 & 11.29439 & 0.15712 & 10.28476 \\
\hline 6 & 0.40147 & 7.573431 & 0.288195 & 7.528819 & 0.194268 & 10.6161 & 0.17328 & 7.763805 \\
\hline 7 & 0.431876 & 5.609651 & 0.309893 & 6.170862 & 0.214891 & 6.977586 & 0.186733 & 7.081021 \\
\hline 8 & 0.456102 & 5.722515 & 0.329016 & 5.712105 & 0.229885 & 6.25421 & 0.199956 & 5.338114 \\
\hline 9 & 0.482203 & 5.631639 & 0.347809 & 5.150106 & 0.244263 & 4.412728 & 0.210629 & 6.192659 \\
\hline 10 & 0.509359 & 4.9305 & 0.365722 & 3.585257 & 0.255042 & 5.49478 & 0.223673 & 5.87942 \\
\hline 11 & 0.534473 & 3.64471 & 0.378834 & 3.095887 & 0.269056 & 2.972104 & 0.236824 & 4.572972 \\
\hline 12 & 0.553953 & 3.466731 & 0.390562 & 2.867638 & 0.277052 & 3.724465 & 0.247654 & 4.920917 \\
\hline 13 & 0.573157 & 2.55033 & 0.401762 & 4.299307 & 0.287371 & 5.363512 & 0.25984 & 3.894514 \\
\hline 14 & 0.587774 & 4.043249 & 0.419035 & 4.146275 & 0.302784 & 1.397303 & 0.26996 & 0.77198 \\
\hline 15 & 0.611539 & 3.031073 & 0.436409 & 3.414988 & 0.307015 & 3.046546 & 0.272044 & 1.973855 \\
\hline 16 & 0.630075 & 3.352772 & 0.451313 & 3.070777 & 0.316368 & 3.020353 & 0.277414 & 3.199496 \\
\hline 17 & 0.6512 & 3.021506 & 0.465172 & 1.93054 & 0.325924 & 1.721481 & 0.28629 & 2.843528 \\
\hline 18 & 0.670877 & 2.104178 & 0.474152 & 2.401292 & 0.331534 & 2.66104 & 0.29443 & 3.489192 \\
\hline 19 & 0.684993 & 1.619538 & 0.485538 & 1.710754 & 0.340357 & 2.396056 & 0.304704 & 3.236004 \\
\hline 20 & 0.696087 & & 0.493844 & & 0.348512 & & 0.314564 & \\
\hline
\end{tabular}




\section{APPENDIX E}

\section{STEPS FOR NORMALIZATION OF DTMs}

E.1 Steps for winsorizing and normalization of curvature DTMs employing raster calculator utility of ArcGIS

1 Partition curvature DTM in to convex and concave DTMs

a) Convexity:

$\mathrm{CON}([$ curvatureDTM] $>=0$, [curvatureDTM $])$

(Output $=$ convexDTM)

b) Concavity:

$\operatorname{CON}([$ curvatureDTM $]<=0$, [curvatureDTM] $)$

(Output $=$ concaveDTM)

Getvalue [percentile 95\%] implemented in TNTmips and excell

\section{Winsorization:}

a) $\mathrm{CON}([$ convexDTM] $>=\operatorname{val}(95 \%$ percentile $), \operatorname{val}(95 \%$ percentile $)$, [9maxx.asc] $)$

(Output $=$ convexDTMwins)

b) $\mathrm{CON}([$ concaveDTM] < $<\operatorname{val}(95 \%$ percentile $), \operatorname{val}(95 \%$ percentile $)$, [9maxx.asc])

(Output $=$ concaveDTMwins)

\section{Normalisation}

a) Convexity

([convexDTMwins] - $\min$ ) / $\max -\min$ )

Output $=$ convexDTMwins_norm

b) Concavity

([concaveDTMwins] - $\max ) /(\min -\max )$

Output $=$ concaveDTMwins_norm

4 Recombination of convexity and concavity DTMs

CON([curvatureDTM] >= 0, [convexDTMwins_norm], [concaveDTMwins_norm]) Output $=$ curvatureDTMwins_norm

Steps for winsorizing and normalization slope employing raster calculator utility of ArcGIS

E.2: Steps for winsorizing and normalization of slope employing raster calculator utility of ArcGIS

1 eliminate flat areas 
$\operatorname{CON}([$ slope $]>=3$, [slope $])$

$($ Output $=$ slope_i)

Get mean value [slope_i]

Output: mean

2 Winsorization:

$\mathrm{CON}([$ slope $]<$ mean, [slope], mean $)$

Out: slope_wins

3 normalize betw $0-1$

([slope_wins] - $\min ) /(\max -\min )$

Out: slope_wins_norm

E.3: Steps for standardization and normalization of TPI employing raster calculator utility of ArcGIS

\section{Standardize TPI}

([TPI] - mean) / std. dev

Out: TPI_std

2 Partition TPI into positive and negative values

a) Positive

CON([TPI_std $]>=0$, [TPI_std $])$

(Output $=$ TPI_std_high)

b) Negative

CON ([TPI_std $]<=0$, [TPI_std $])$

(Output $=$ TPI_std_low)

3 Normalisation

a) Highness

([TPI_std_high] - min) / $\max -\min$ )

Output $=$ TPI_std_high_norm

b) Lowness

([TPI_std_low] - max $) /(\min -\max )$

Output $=$ TPI_std_low_norm

4 Recombination of positive and negative TPIs

CON([TPI] $>=$ 0, [TPI_std_high_norm], [TPI_std_low_norm])

Output $=$ TPI_std_norm 


\section{APPENDIX F}

\section{VALIDATION FORM}

Intention of this form is to give basic information about the method to help compare the classification results vs. geoscientist's point of view of random point locations given the class descriptions classification strategy and the scale of the study. Last page include instructions for the implementation.

\section{Method:}

Proposed method aims at classifying the terrain into landform classes based on a general purpose landform classification scheme. Terrain is classified into form elements based local geometry. Following this procedure a higher level context is incorporated via relative position and terrain structure information to reorganise form elements into geomorphometric landform classes.

\section{Local geometry:}

Landforms can be classified according to their local geometry.

Slope and four curvature parameters namely; plan curvature, profile curvature, maximum curvature and minimum curvature were used to classify terrain into 15 form elements. Form Element model of Dikau (1989) was adopted for the classification. Accordingly form is a basic component to identify the landforms.
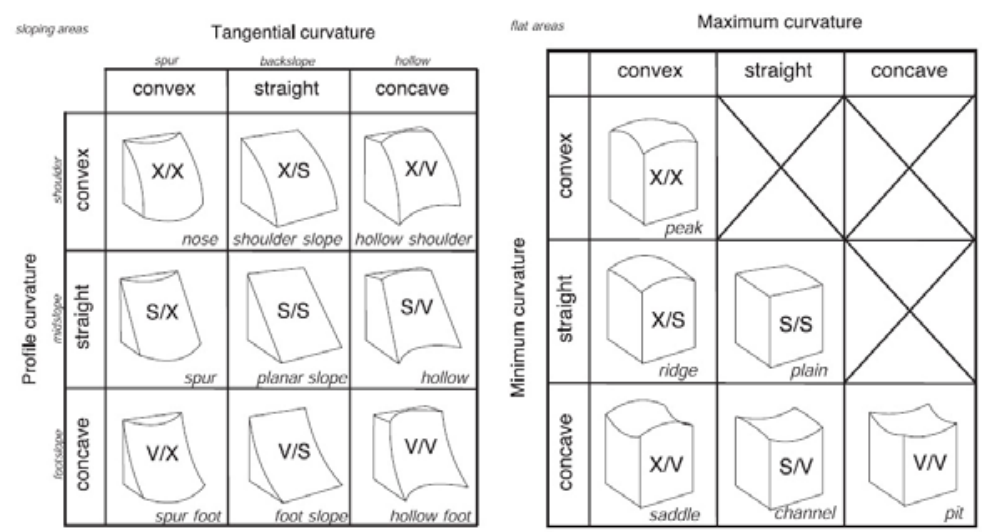

Fifteen ‘form elements' offered by Dikau (1989) based on local geometry

\section{Relative Position:}

Landforms are not chaotic across the landscape but they are rather organized. E.g. a ridge or a peak has a relatively higher position compared to all other forms where as channel is located at relatively lower positions, other sloping form elements are organised in between these extremes. Shoulders and noses tend to be at higher positions where curved forms like Foot slopes remain at the lower parts. 


\section{Terrain Structure}

Surface specific linear features; namely basin divides and flow paths were used to discriminate certain features i.e. ridges, peaks and channels and to establish connectivity.

\section{Classes and Class Descriptions:}

There a re many nomenclature and definitions about the classes of interest in the literature. Some definitions regard specific domain, e.g. geology, surface hydrology, etc however those class names are also generic and relate to human perception. Therefore it is thought that visual descriptions would better describe the classes of interest.

Good representatives of those classes have been captured from map scene. Topographic contour lines (10m vertically spaced) overlaid on shaded relief of a hilly terrain (one of the case areas) for $1 \times 1 \mathrm{~km}$ sample areas were captured. First group of features (Figure 2) relate to non-sloping landforms and second group of features relate to the sloping landforms (Figure 3) and all represented in symbolic shapes in the previous figure (Figure1).

Though some of the landforms are very rare compared to others like for instance there are very few pits and saddles where plenty of sloping elements like planar slopes, hollows and spurs exist.

Some landforms in Figure 1 are known as surface specific features, namely; peaks, ridges and channels, they construct the skeleton of the terrain. Some landforms are accompanying landforms such as; shoulders usually appear at the two sides of a ridge or spur. Foot slopes are positioned at the two sides of a channel and at the lower end of a hillslope.

Some landforms are within a typical arrangement like hollows and spurs appear in sequence on a hillslope.

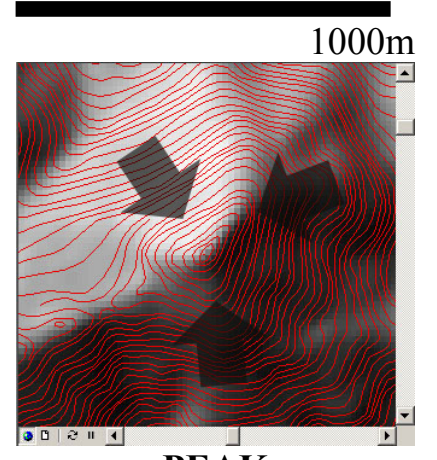

PEAK

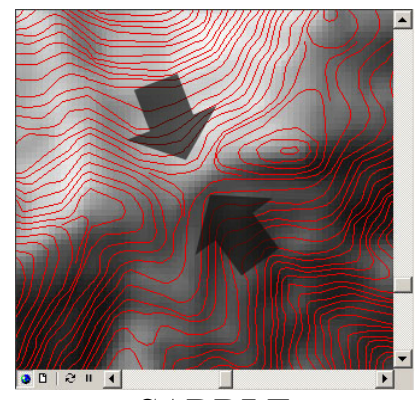

SADDLE

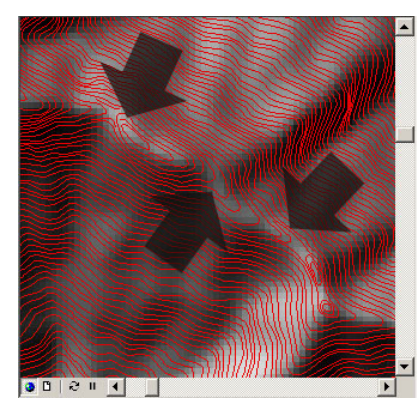

RIDGE

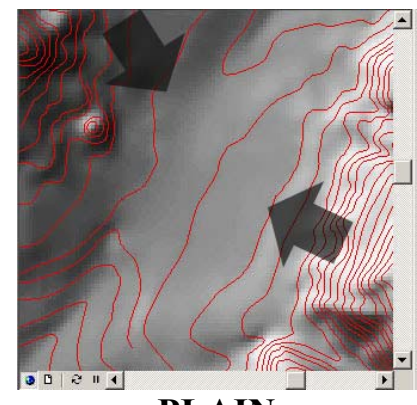

PLAIN

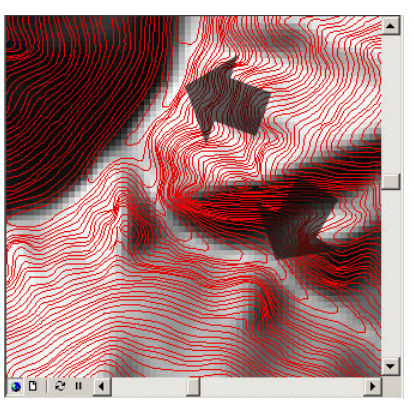

CHANNEL

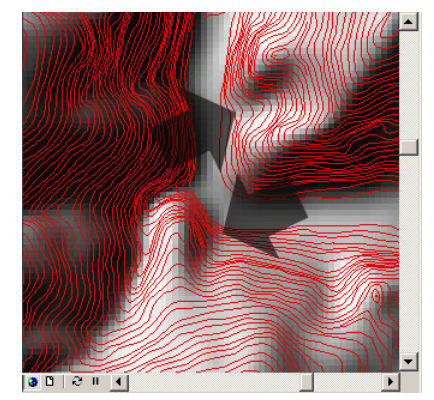

PIT

Representative features that pertain to non-sloping part of the land surface 

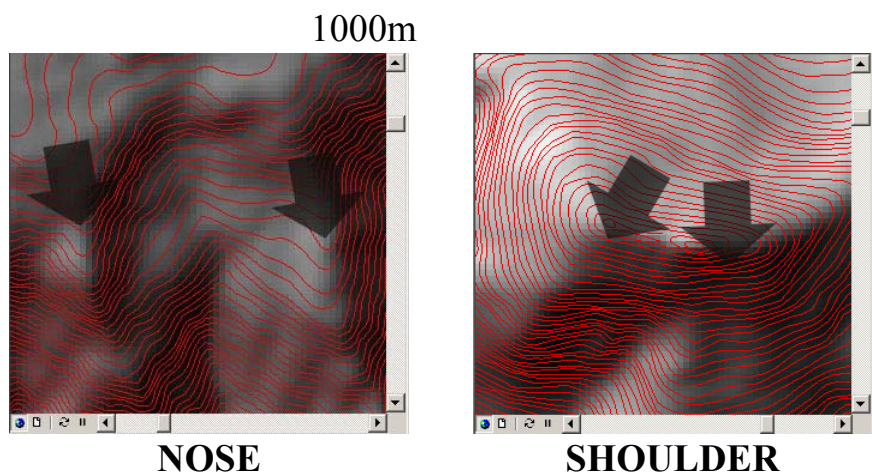

SHOULDER

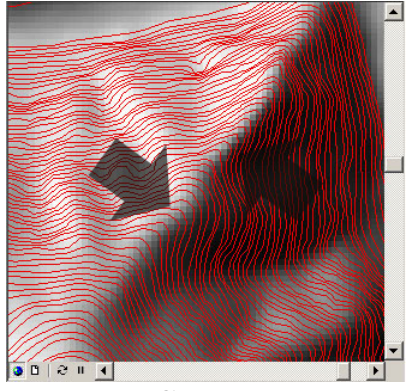

SPUR

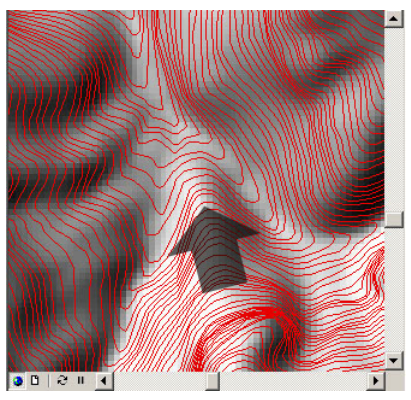

SPUR FOOT

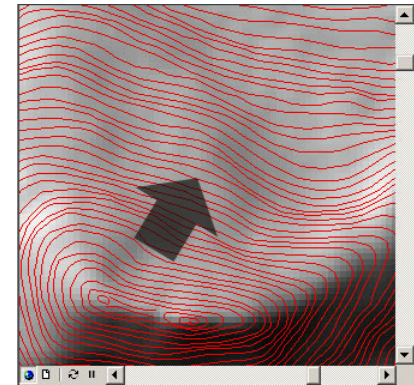

PLANAR SLOPE

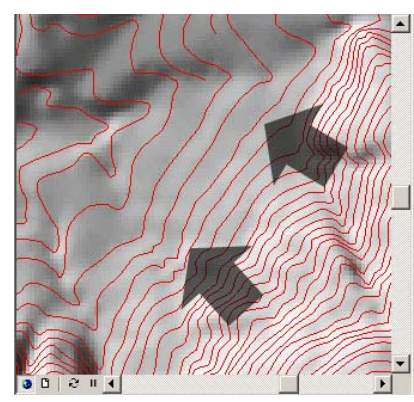

FOOT SLOPE

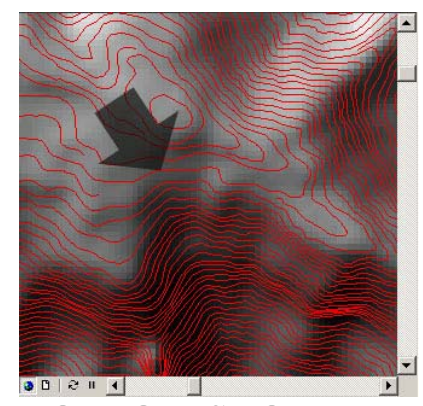

HOLLOW SHOULDER

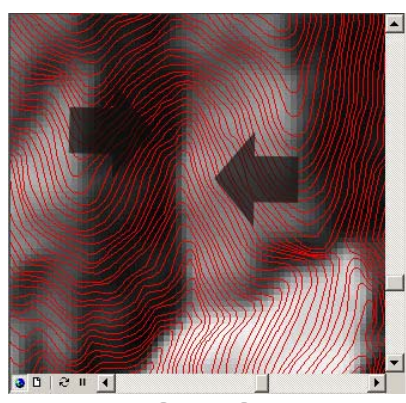

HOLLOW

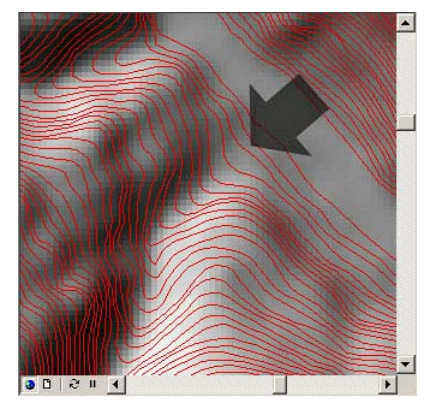

HOLLOW FOOT

Representative features that pertain to non-sloping part of the land surface

\section{What will you do?}

Method is applied on two case areas of $15 \times 15 \mathrm{~km}$ which significantly represents afore mentioned classes.

A total number of $\mathbf{8 5}$ stratified random points for each case area were generated to validate the results. You are simply asked to assign each point into one of the below classes.

$\begin{array}{ll}\text { Ridge } & \text { Nose } \\ \text { Peak } & \text { Spur } \\ \text { Saddle } & \text { Spur foot } \\ \text { Plain } & \text { Shoulder slope } \\ \text { Channel } & \text { Planar slope } \\ \text { Pit } & \text { Foot slope } \\ & \text { Hollow shoulder } \\ & \text { Hollow } \\ & \text { Hollow foot }\end{array}$


1.

Open "case_1.mxd" for case1 and open "case_2.mxd" for case2"in ArcGIS.

There you will find all necessary data for filling the database table provided.

Reference data provided includes the necessary layers to understand terrain characteristics and scale of the study. There are two sets one for each case area.

Those sets include:

- Relief: Shaded relief of the terrain

- Anudem15: DEM (Original 15m resolution DEM of the study area interpolated from contours using topogrid ArcGIS.

- Contour: $10 \mathrm{~m}$ vertically spaced contour data of the area. Please use this layer if you have difficulty in interpreting relief.

- Kernel mesh: A grid mesh that represent kernel size used for calculating the DTMs in order to describe scale of the study

- Objects: Segmentation result that is generated before classification in order to help understanding homogeneous form objects and extents of the objects to be classified.

- Random points: point data used for validation purposes. 85 random points generated by stratified random sampling, so that even classes of small extents are assigned with points. They have the id labels displayed (0 to 84). You can select the point on the attribute table and then "zoom to selected" for simplicity.

2.

Open "db_case1.mdb" with Microsoft Access, then open "table_case1"

* If you get a security warning you can safely ignore it (it is due to a code in the table relation)

for case2: open db_case2.mdb" then open "table_case2"

You will see point records with id numbers ( 0 to 84$)$ in the first field. They correspond to the same id numbers as in point data in the reference data set. You will simply assign each record a class name by selecting one of the 15 classes from the dropdown menu in the "LanformClass" tab"

e.g. For point on the map with id $=0$, go to access database and from table go to record with $\mathrm{ID}=0$, on the next tab "LanformClass" select its appropriate class from the dropdown list. Select the most similar if you can't decide about the class, or leave it blank.

Please save the mdb file, you can rename it including your name (optionally) and email it to denizger@metu.edu.tr

(only mdb file is enough)

Thank you very much, Deniz. 


\section{VITA}

\section{PERSONAL INFORMATION}

\section{Deniz Gerçek}

Date and Place of Birth: 6.10.1975, Kayseri

Nationality: Turkish (TC)

Marital Status: Single

Phone: +90 5334166029

email: denizger@metu.edu.tr, denizger@gmail.com

\section{EDUCATION}

Ph.D Geodetic and Geographic Information Technologies, The Graduate

School of Natural and Applied Sciences, Middle East Technical University (M.E.T.U.), Ankara, Turkey

M.Sc Geodetic and Geographic Information Technologies, The Graduate School of Natural and Applied Sciences, Middle East Technical University (M.E.T.U.), Ankara, Turkey

B.Sc. City and Regional Planning, Faculty of Architecture, Gazi University, Ankara, Turkey

\section{WORK EXPERIENCE}

METU - Middle East Technical University HAT Geographic Information Systems Inc. Atacan City Planning Inc. Iller Bank

\section{FOREIGN LANGUAGES}

Advanced English, Basic German

\section{PUBLICATIONS}

GERÇEK D., ZEYDANLI U., 2010, "Object-based classification of Landscape into Land management Units (LMUs), Geographic Object based Image Analysis, GEOBIA 2010 Ghent, Belgium (to be published)

GERÇEK D., 2009, "GIS Applications and Spatial Analysis in Archaeology", TOPOI Workshop: Zeugma - New Excavation Results and Methods of Research, May 13-14, 2009, Freie Universitat, Berlin, Germany

GERÇEK D., 2009, "Object-based classification of landforms based on their local geometry and geomorphometric context", Object-Based Landscape Analysis, RSPSoc Land Cover/Land Use SIG, 7-8 April 2009, University of Nottingham, UK

GERÇEK D., 2006, Mapping Land Cover on a Continental Region with the use of Remotely Senesed and Digital Terrain Data, International Symposium on Terrain Analysis and Digital Terrain Modelling (TADTM), 2006, Nanjing, China, Symposeum Proceeding p.49

GERÇEK D., 2004, Improvement of Image Classification with the Integration of Topographical Data, ISPRS 2004, 12-23 July 2004, Symposium Proceeding, p.53-58 (published as DVD)

GERÇEK D., YILDIRIM T., ARCASOY A., TOPRAK V., 2003 "Lineament Analysis of the Eruption Centers of Galetean Volcanics (Turkey) and its Relation to North Anatolian Fault System" Int. Workshop on the North Anatolian, East Anatolian and Dead Sea Fault System, METU, Ankara. 